\title{
Strangeness in nuclear physics
}

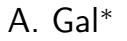 \\ Racah Institute of Physics, \\ The Hebrew University, \\ Jerusalem 91904, \\ Israel \\ E. V. Hungerford ${ }^{\dagger}$ \\ University of Houston, \\ Houston, TX 77204 , \\ USA \\ D. J. Millener \\ Brookhaven National Laboratory, \\ Upton, NY 11973, \\ USA
}

Extensions of nuclear physics to the strange sector are reviewed, covering data and models of $\Lambda$ and other hypernuclei, multistrange matter, and antikaon bound states and condensation. Past achievements are highlighted, present unresolved problems discussed, and future directions outlined.

PACS numbers: 13.75.Ev, 13.75.Jz, 21.80.+a, 25.80.-e, 26.60.+c, 97.60.Jd

\section{CONTENTS}

I. Introduction

A. Brief historical overview

B. General features of hypernuclear structure

1. Kinematics

2. Examples - kinematic considerations of hypernuclear production

C. The distorted wave impulse approximation

D. Continuum excitations

E. The nuclear Auger effect

F. Strangeness production: reactions and experimental techniques

1. The $\left(K_{\text {stop }}^{-}, \pi^{-}\right)$reaction

2. The $\left(K_{\text {stop }}^{-}, \pi^{0}\right)$ reaction

3. The in-flight $\left(K^{-}, \pi^{-}\right)$reaction

4. The $\left(\pi^{+}, K^{+}\right)$reaction

5. The $\left(e, e^{\prime} K^{+}\right)$reaction

6. Single-particle structure

7. $\left(K^{-}, K^{+}\right)$and stopped $\Xi^{-}$reactions

8. Hypernuclear lifetime measurements

G. Free-space and in-medium $Y N$ interactions

1. The free-space $Y N$ interaction

2. Extraction of $\Lambda N$ interaction in final-state interactions

3. Comparison of the $\Lambda N$ and $\Sigma N$ interactions

4. The effective $Y N$ interaction

II. $\Lambda$ Hypernuclei

A. Structure calculations

1. $s$-shell hypernuclei

$\begin{array}{ll}* & \text { avragal@vms.huji.ac.il } \\ \dagger & \text { hunger@uh.edu } \\ \ddagger & \text { millener@bnl.gov }\end{array}$
2. $p$-shell hypernuclei, $\gamma$-ray measurements, and spin dependence of the $\Lambda N$ interaction 23

B. Weak decays of $\Lambda$ hypernuclei

1. Mesonic decays 30

2. Nonmesonic decays

III. $\Sigma$ Hypernuclei

A. Overview

B. $\Sigma$ nuclear potentials from fits to $\Sigma^{-}$atoms 37

C. Evidence from $\left(\pi^{-}, K^{+}\right)$spectra

IV. $\Lambda-\Lambda$ Hypernuclei

V. $\Xi$ Hypernuclei 41

VI. Strange Dense Matter 42

A. Strange hadronic matter 42

B. Neutron stars

VII. $\bar{K}$-Nuclear Interactions and Bound States 46

VIII. Future Experiments and Directions

A. Spectroscopy using meson beams 50

1. Hyperon production and hyperon-nucleon
interactions

2. Reaction spectroscopy with mesons 51

3. Experiments using emulsion detectors 51

4. Spectroscopy using electromagnetic transitions 51

B. Spectroscopy with electron accelerators 55

1. Electroproduction at Mainz (MAMI)

2. Electroproduction at Jlab

C. Experiments at PANDA

D. Weak decay of hypernuclei

1. mesonic decays

2. nonmesonic decays 54

3. $\Lambda$ hypernuclear lifetimes

E. Multi-strange systems

F. Experiments at heavy-ion facilities 56

G. $\bar{K}$-nucleus bound-state searches 
IX. Summary

Acknowledgments

References

\section{INTRODUCTION}

\section{A. Brief historical overview}

In the early 1950s a quantum number, conserved under the strong interaction, was introduced (Gell-Mann, 1953 , Nakano and Nishijima, 1953) in order to explain the behavior of the "strange" particles which had been observed in emulsions exposed to cosmic rays. Almost simultaneously, the first hypernucleus, formed by a $\Lambda$ hyperon bound to a nuclear fragment, was observed in an emulsion exposed to cosmic rays (Danysz and Pniewski, 1953). For the next 20 years or so, hypernuclei were explored using emulsion detectors, first with cosmic rays, and then with beams from existing accelerators. Within the last 40 years, modern particle accelerators and electronic instrumentation has increased the rate and breadth of the experimental investigation of strangeness in nuclei. As always, theoretical interest has closely followed the experimental development.

The behavior of a $\Lambda$ in a nuclear system is a nuclear many-body problem, since the forces between the baryons are predominantly hadronic and the time scale of the strong interaction is about $10^{-23} \mathrm{~s}$ compared to the weak-interaction lifetime of a $\Lambda$ in the nuclear medium (Bhang et al. 1998, Park et al., 2000) of approximately $10^{-10} \mathrm{~s}$. Therefore, the combined hypernuclear system can be treated using well developed nucleartheory models such as the shell or mean-field models with an effective $\Lambda$-nucleus interaction. New dynamical symmetries may also arise in hypernuclei, e.g. by treating the $\Lambda$ hyperon shell-model orbitals on par with those of nucleons within the Sakata version of $\mathrm{SU}(3)$ symmetry (Sakata, 1956). This approach was found useful in hypernuclear spectroscopic studies (Auerbach et al. 1981 , 1983). Furthermore, by coupling SU(3)-Sakata with $\mathrm{SU}(2)$-spin, the resulting $\mathrm{SU}(6)$ symmetry group presents a natural extension of Wigner's SU(4) spin-isospin symmetry group in light nuclei (Dalitz and Gal, 1981).

$\Lambda$ hypernuclei also offer a test-ground for microscopic approaches to the baryon-baryon interaction. Thus, since one-pion exchange (OPE) between a $\Lambda$ hyperon and a nucleon is forbidden by isospin conservation, the $\Lambda N$ interaction has shorter range, and is dominated by higher mass (and multiple) meson exchanges when compared to the $N N$ interaction. For example, two-pion exchange between a $\Lambda$ hyperon and a nucleon proceeds through intermediate $\Sigma N$ states $(\Lambda N \rightarrow \Sigma N \rightarrow \Lambda N)$, potentially leading to non-negligible three-body $\Lambda N N$ forces (Gibson and Lehman, 1988). The analogous mechanism of intermediate $\Delta N$ states $(N N \rightarrow \Delta N \rightarrow N N)$ in generating three-body $N N N$ forces in two-pion exchange (Epelbaum, Hammer, and Meißner, 2009) seems to be less important in nuclear physics, not only because the $N N$ interaction is dominated by OPE, but also because of the considerably higher excitation mass of the $\Delta$ resonance with respect to that of the $\Sigma$ hyperon. Such theoretical expectations may be explored in hypernuclear few-body and spectroscopic calculations.

Finally, the $\Lambda$ can be used as a selective probe of the nuclear medium, providing insight into nuclear properties that cannot be easily addressed by other techniques. Thus, from a hadronic as opposed to a quark perspective, the $\Lambda$ remains a distinguishable baryon within the nucleus, and samples the nuclear interior where there is little direct information on the single-particle structure of nuclei. Because of this, various aspects of hypernuclear studies such as $\Lambda$ decay, or the spectra of heavy hypernuclear systems, can illuminate nuclear features which would be more obscured in conventional nuclei.

Useful material on the subject of this review can be found in the proceedings of the recent triennial conferences on Hypernuclear and Strange Particle Physics (Gal and Hungerford, 2005; Pochodzalla and Walcher, 2007; Gibson et al., 2010; Juliá-Diaz et al., 2013), special volumes (Motoba, Akaishi, and Ikeda, 1994; Gal and Hayano, 2008; Hiyama, Motoba, and Yamamoto, 2010b; Gal, Hashimoto, and Pochodzalla, 2012), schools (Bydžovský, Gal, and Mareš, 2007), and several review articles (Hashimoto and Tamura, 2006; Botta, Bressani, and Garbarino, 2012; Feliciello and Nagae, 2015).

\section{B. General features of hypernuclear structure}

To review nomenclature, a hypernucleus is constructed from a normal nucleus, with atomic weight $\mathrm{A}$ and atomic number Z, by adding one or more bound hyperons $(\Lambda, \Sigma$, $\Xi$, and perhaps $\Omega$ ). For example, the hypernucleus ${ }_{\Lambda}^{12} \mathrm{C}$ consists of 12 baryons, one of these being a $\Lambda$ hyperon. It has atomic number 6 , as noted by the label C. However for a general hypernucleus, the atomic number identifier is a measure of the system charge, and not necessarily the number of protons, since hyperons can carry charge.

A hypernucleus is characterized by its spin, isospin, and in the case of $\Lambda$ hypernuclei, a strangeness of -1 . If the $\Lambda$ is injected into the nuclear system, the resulting hypernucleus will normally de-excite by a nuclear Auger process, or by $\gamma$ emission. The resulting ground state then decays by the weak interaction, emitting $\pi$ mesons as in the free $\Lambda$ decay, and also nucleons in a four-fermion in-medium interaction $\Lambda N \rightarrow N N$. Therefore, observation of the energetics of hypernuclear formation and decay can provide information on binding energies and spins of hypernuclear ground states. To conserve baryon number, a reaction producing a hypernucleus commonly 
TABLE I Experimental $\Lambda$ separation energies, $B_{\Lambda}$, of light hypernuclei from emulsion studies. These are taken from a compilation (Davis and Pniewski, 1986) of results from (Cantwell et al. , 1974 Jurič et al., 1973 ), omitting ${ }_{\Lambda}^{15} \mathrm{~N}$ (Davis, 1991). A reanalysis for ${ }_{\Lambda}^{12} \mathrm{C}$ (Dłuzewski et al. 1988 gives 10.80(18) MeV.

\begin{tabular}{lcc}
\hline \hline Hypernucleus & Number of events & $B_{\Lambda} \pm \Delta B_{\Lambda}(\mathrm{MeV})$ \\
\hline${ }_{\Lambda}^{3} \mathrm{H}$ & 204 & $0.13 \pm 0.05$ \\
${ }_{\Lambda}^{4} \mathrm{H}$ & 155 & $2.04 \pm 0.04$ \\
${ }_{\Lambda}^{4} \mathrm{He}$ & 279 & $2.39 \pm 0.03$ \\
${ }_{\Lambda}^{5} \mathrm{He}$ & 1784 & $3.12 \pm 0.02$ \\
${ }_{\Lambda}^{6} \mathrm{He}$ & 31 & $4.18 \pm 0.10$ \\
${ }_{\Lambda}^{7} \mathrm{He}$ & 16 & not averaged \\
${ }_{\Lambda}^{7} \mathrm{Li}$ & 226 & $5.58 \pm 0.03$ \\
${ }_{\Lambda}^{7} \mathrm{Be}$ & 35 & $5.16 \pm 0.08$ \\
${ }_{\Lambda}^{8} \mathrm{He}$ & 6 & $7.16 \pm 0.70$ \\
${ }_{\Lambda}^{8} \mathrm{Li}$ & 787 & $6.80 \pm 0.03$ \\
${ }_{\Lambda}^{8} \mathrm{Be}$ & 68 & $6.84 \pm 0.05$ \\
${ }_{\Lambda}^{9} \mathrm{Li}$ & 8 & $8.50 \pm 0.12$ \\
${ }_{\Lambda}^{9} \mathrm{Be}$ & 222 & $6.71 \pm 0.04$ \\
${ }_{\Lambda}^{9} \mathrm{~B}$ & 4 & $8.29 \pm 0.18$ \\
${ }_{1} \mathrm{~B} \mathrm{Be}$ & 3 & $9.11 \pm 0.22$ \\
${ }_{\Lambda}^{10} \mathrm{~B}$ & 10 & $8.89 \pm 0.12$ \\
${ }_{\Lambda}^{11} \mathrm{~B}$ & 73 & $10.24 \pm 0.05$ \\
${ }_{\Lambda}^{12} \mathrm{~B}$ & 87 & $11.37 \pm 0.06$ \\
${ }_{\Lambda}^{12} \mathrm{C}$ & 6 & $10.76 \pm 0.19$ \\
${ }_{1}^{13} \mathrm{C}$ & $6.69 \pm 0.12$ \\
${ }_{\Lambda}^{14} \mathrm{C}$ & 6 & $11.69 \pm 0.33$ \\
\hline \hline
\end{tabular}

replaces a nucleon with a $\Lambda$. In terms of the shell model, a hypernucleus is then described by a set of $\Lambda N^{-1}$ particlehole excitations of the target nucleus which are coupled to specific values of spin and isospin.

The acquisition of hypernuclear binding energies, welldepths, and positions of the hypernuclear levels began in the 1960s. Early work included $K^{-}$absorption in emulsions and bubble chambers, where hyperfragments were identified by their mesonic decays. These efforts successfully established the binding energies of a number of light hypernuclei in their ground states (g.s.) where the $\Lambda$ is in the lowest $s_{1 / 2}$ orbit, as summarized in Table I. In 1972, the existence of a ${ }_{\Lambda}^{12} \mathrm{C}$ particle-unstable state with a $\Lambda$ in the $p$ orbit was confirmed (Jurič et al., 1972$)$, and the reaction $K^{-}+{ }^{12} \mathrm{C} \rightarrow \pi^{-}+p+{ }_{\Lambda}^{11} \mathrm{~B}$ in emulsion was used to study excited states of ${ }_{\Lambda}^{12} \mathrm{C}$. Beginning in the mid 1970's, the structure of $p$-shell hypernuclei was further explored via $\left(K^{-}, \pi^{-}\right)$reactions using accelerated beams of kaons and magnetic spectrometers. Binding energies of heavier hypernuclear systems were extracted from spectra obtained using the $\left(\pi^{+}, K^{+}\right)$reaction. This reaction has greater probability to populate interior states. Unfortunately, the mass (or binding energy) scale for most of the data was normalized to the emulsion $B_{\Lambda}$ value (Table I for ${ }_{\Lambda}^{12} \mathrm{C}$ that is determined by only a few events. This, coupled with resolution issues in the reaction spectra, lead to some uncertainties

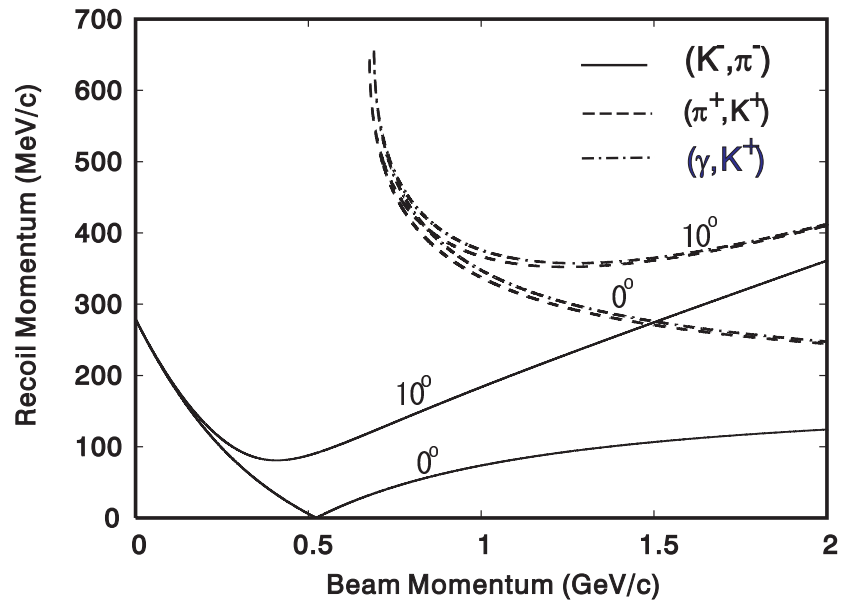

FIG. 1 The recoil momentum of the $\Lambda$ hypernucleus produced from a ${ }^{12} \mathrm{C}$ target, as a function of the incident particle momentum and angle for several production reactions.

in binding energies. Some of the binding-energy uncertainties have been sorted out in recent years by comparing with $\left(e, e^{\prime} K^{+}\right)$electroproduction measurements (see Sec. I.F.6.

\section{Kinematics}

The kinematics for several elementary reaction processes are shown in Fig. 1. As indicated in the figure, the $\left(K^{-}, \pi^{-}\right)$reaction can have low, essentially zero momentum transfer to the produced $\Lambda$ hypernuclei. This also holds for $\Sigma$ hypernuclei. Thus the probability is large that $\Lambda$ and $\Sigma$ hyperons, when produced at low momentum transfer, will interact with, and bind to, the residual spectator nucleus. On the other hand reactions such as $\left(\pi^{+}, K^{+}\right)$or $\left(\gamma, K^{+}\right)$have high momentum transfer with respect to the nuclear Fermi momentum, producing recoil hyperons that have a high probability of escaping the nucleus. Such reactions are loosely termed "quasifree" (QF) processes, although the hyperon actually experiences continuum, final-state interactions (FSI). Obviously, in the case of higher momentum transfer, cross sections to bound states are significantly reduced.

Furthermore, a $K^{-}$strongly interacts with nucleons through various resonant states. Thus incident kaons in a $\left(K^{-}, \pi^{-}\right)$reaction attenuate rapidly in nuclear matter, and the transition density should peak at the nuclear surface to maximise the cross section. Combining this with low momentum transfer as discussed above, the $\left(K^{-}, \pi^{-}\right)$reaction most likely involves an outer shell neutron, simply replacing this neutron with a $\Lambda$ having the same single-particle quantum numbers. On the other hand, energetic $\pi^{+}$and $K^{+}$particles have longer meanfree paths in nuclear matter, and give larger momentum transfer to the hyperon. Thus they can interact with interior nucleons, and can impart significant angular mo- 


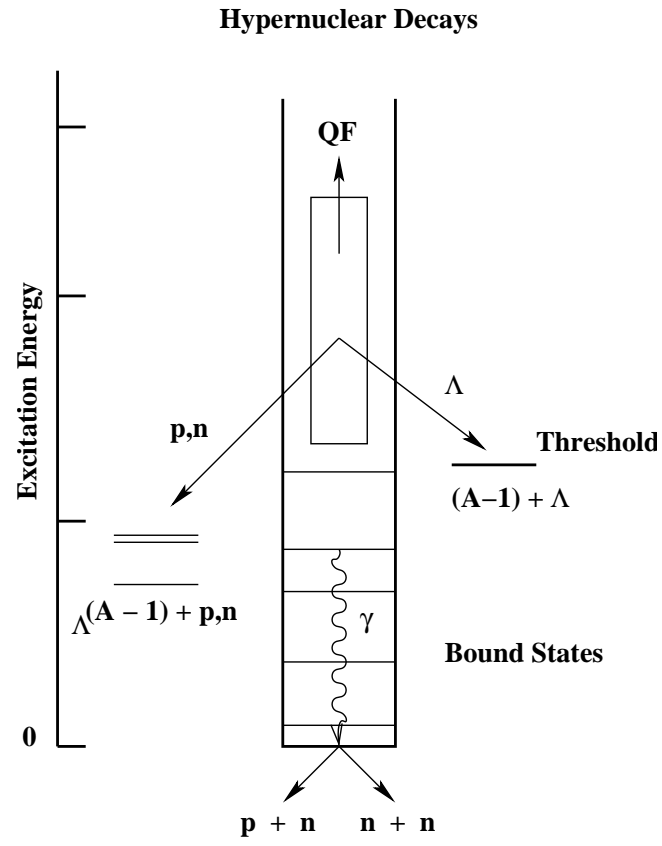

FIG. 2 A schematic representation of the decays of an excited hypernucleus, showing in particular the decay of highly excited states by Auger and $\gamma$-ray transitions.

mentum transfer. However, such reactions have greater quasi-free strength.

After production, a bound hypernucleus generally deexcites to the state in which all the baryons reside in their lowest single-particle levels, from which the hypernuclear ground state then decays via the weak interaction. The energy released in the nuclear transitions is removed by gamma rays, or Auger neutron emission (see Fig. 2) because the neutron (or proton) emission threshold can be lower than the $\Lambda$ emission threshold. Above the $\Lambda$ threshold, $\Lambda$ as well as nucleon emission can occur. It is interesting to note that particle-unstable hypernuclear levels near $B_{\Lambda}=0$ are experimentally observed to have narrow widths. Nuclear states at comparable excitation energies would be broad. However, the narrow width of $\Lambda$-nuclear states is due to the weakness of the $\Lambda N$ interaction relative to the $N N$ interaction (Likar, Rosina, and Povh, 1986).

\section{Examples - kinematic considerations of hypernuclear production}

The $\left(K_{\text {stop }}^{-}, \pi^{-}\right)$reaction was the first reaction used for hypernuclear production, as kaon beams, particularly those produced in early accelerator experiments, were weak and the intensity of pions in the beams obscured the production reaction pions. Thus, it was easier to identify a stopped $K^{-}$, and stopping the $K^{-}$assured that essentially all the kaons interacted with the target. As discussed earlier, this reaction was used with an emulsion detector, to produce an excited complex of states in ${ }_{\Lambda}^{12} \mathrm{C}$ which decayed by proton emission to ${ }_{\Lambda}^{11} \mathrm{~B}$. In this case, the emitted proton energy was measured in the emulsion, and the level structure interpreted in terms of three $p$-shell $\Lambda$ states located at about $11 \mathrm{MeV}$ excitation energy (Dalitz, Davis, and Tovee, 1986). These included a narrow state with width equal to the experimental resolution $(\approx 100 \mathrm{keV})$ just $140 \mathrm{keV}$ below the ${ }^{11} \mathrm{C}+\Lambda$ threshold. This state was assigned as the expected $0^{+}$state (Dalitz, Davis, and Tovee, 1986, Davis, 2008). Beneath this state was a broader level with a width of $\approx 600 \mathrm{keV}$ which was interpreted as one of the expected $2^{+}$states. The third state, $750 \mathrm{keV}$ below the second state, had a width of $\approx 150 \mathrm{keV}$ and was also interpreted as another $2^{+}$state.

Later, it was recognized that the incident momentum of the in-flight ${ }^{A} Z\left(K^{-}, \pi^{-}\right){ }_{\Lambda}^{A} \mathrm{Z}$ reaction could be chosen so that the momentum transferred to the hypernucleus is close to zero, Fig. 1, and that kaon beams near 750 $\mathrm{MeV} / \mathrm{c}$ provide a maximum in the elementary cross section. Thus, using this reaction, a series of experiments were initiated at CERN (Povh, 1980) and then at BNL (Chrien et al. 1979, May et al., 1981). The spectra produced by the $\left(K^{-}, \pi^{-}\right)$experiments show peaks for substitutional states near the nuclear surface (i.e., a neutron replaced by a $\Lambda$ with the same quantum numbers).

In the case of $\Sigma$ production (Dover, Gal, and Millener, 1984), the $N\left(K^{-}, \pi\right) \Sigma$ differential cross section in the forward direction shows two enhancements, one at about $400 \mathrm{MeV} / \mathrm{c}$ and a smaller one of different isospin at about $750 \mathrm{MeV} / \mathrm{c}$. A $400 \mathrm{MeV} / \mathrm{c}$ momentum is generally too low to be useful, since the intensity of secondary kaon beams drops rapidly below $600 \mathrm{MeV} / \mathrm{c}$. On the other hand, zero momentum transfer occurs at an incident kaon momentum of about $300 \mathrm{MeV} / \mathrm{c}$, and QF production is significantly enhanced if the incident momentum is greater than $600 \mathrm{MeV} / \mathrm{c}$. However, there have been several searches for $\Sigma$ hypernuclei using very low momentum kaon beams (Bertini et al. 1980, 1984, 1985). Finally, there is another enhancement in the elementary $N\left(K^{-}, \pi\right) \Lambda$ cross section at about $1.7 \mathrm{GeV} / \mathrm{c}$. This momentum range, bearing some promise of appreciable polarization, has been used recently in $\left(K^{-}, \pi^{-} \gamma\right)$ experiments (J-PARC E13) using a $1.5 \mathrm{GeV} / \mathrm{c}$ beam from the J-PARC K1.8 beam line.

\section{The distorted wave impulse approximation}

Obviously to produce a hypernucleus, one needs to bind the hyperon in a nuclear potential well. This potential is usually generated by fitting its depth to some known $\Lambda$ single-particle binding energy in a Woods-Saxon shaped well with geometry derived from nuclear phenomenology. Potential wells for nucleons are often obtained from density-dependent mean-field calculations.

In a simple single-particle model, a production reaction 
removes a nucleon from a nuclear level (nuclear shell) replacing it with a $\Lambda$ in a $\Lambda$ level (shell). Thus, for a closed-shell target nucleus, the structure developed in a $\Lambda N^{-1}$ particle-hole model provides an obvious basis for a theoretical description of the production process. In this model, the production reaction can be described by the distorted wave impulse approximation (DWIA) (Hüfner, Lee, and Weidenmuller, 1974; Bouyssy, 1977). This formulation views the target as a collection of nucleons in single-particle levels, with the amplitude for production occurring between the incident projectile and a nucleon in the target. In this most straightforward treatment of the hypernuclear production cross section, the laboratory cross section for $\left(K^{-}, \pi^{-}\right)$(or similarly $\left(\pi^{+}, K^{+}\right)$) reactions can be written in terms of the two-body cross section on a nucleon in a factorized form as (Itonaga, Motoba, and Sotona, 1994, Motoba, Itonaga, and Yamamoto 2010)

$$
\frac{d \sigma(\theta)}{d \Omega_{L}}=\alpha_{\mathrm{kin}} \frac{d \sigma_{e l}(\theta)}{d \Omega_{L}} N_{\mathrm{eff}}(i f ; \theta)
$$

where $\alpha_{\text {kin }}$ is a kinematic factor involving the energies and momenta of the participants and $N_{\text {eff }}(i f ; \theta)$ is the distorted-wave integral, known as the effective neutron number, defined by

$$
\begin{aligned}
& \frac{1}{2 J_{i}+1} \sum_{M_{i} M_{f}} \mid\left\langle J_{f} M_{f} T_{f} \tau_{f}\right| \int d \boldsymbol{r} \chi_{\pi}^{(-) *}\left(\boldsymbol{k}_{\pi}, \frac{M_{A}}{M_{H}} \boldsymbol{r}\right) \\
& \times\left.\sum_{j=1}^{A} U_{-}(j) \delta\left(\boldsymbol{r}-\frac{M_{C}}{M_{A}} \boldsymbol{r}_{j}\right)\left|J_{i} M_{i} T_{i} \tau_{i}\right\rangle\right|^{2} \chi_{K}^{+}\left(\boldsymbol{k}_{K}, \boldsymbol{r}\right) .
\end{aligned}
$$

$M_{H}\left(M_{A}\right)$ is the hypernuclear (target) mass and $M_{C}$ refers to the nuclear core of the hypernucleus. A zerorange interaction is assumed and the operator $U_{-}$converts a neutron into a $\Lambda$ hyperon. In a more sophisticated treatment that also enables the calculation of hypernuclear polarizations (Itonaga, Motoba, and Sotona, 1994), a term $f+g\left(\boldsymbol{\sigma}_{j} \cdot \widehat{\boldsymbol{n}}\right)$ is included under the summation over $j$ rather than using the two-body cross section in the factorized form of Eq. (1). Here, $f$ and $g$ denote the two-body spin-nonflip and spin-flip amplitudes and $\widehat{\boldsymbol{n}}$ is a unit vector perpendicular to the reaction plane. The BNL group (Auerbach et al. 1983) factors out the square of the Fermi-averaged amplitude $f$.

In Eq. (2), the $\chi$ 's are the distorted incident and final wave functions for the kaon and pion obtained from the nuclear optical potentials. Motoba and collaborators use eikonal distorted waves based on the elementary $K N$ and $\pi N$ cross sections while the BNL group fits the elastic scattering of $800 \mathrm{MeV} / \mathrm{c} \pi$ 's and $K$ 's on ${ }^{12} \mathrm{C}$. For cross sections, the results from the two groups are in quite good agreement.

As for any inelastic scattering involving a one-body transition, the nuclear structure information is encoded in one-body density-matrix elements (OBDME), namely the matrix elements between the initial nuclear and final hypernuclear states of a coupled product of an annihilation operator for the nucleon and a creation operator for the $\Lambda$ (Auerbach et al. 1983). An instructive example occurs when the hypernuclear wave function represents a simple weak-coupling state (this is a reasonable approximation because the $\Lambda N$ interaction is quite weak). Then, the OBDME that governs the cross section is

$$
\begin{aligned}
\left\langle\alpha_{c} J_{c} T_{f}, j_{\Lambda} 0 ; J_{f} T_{f}\left\|\left(a_{j_{\Lambda}}^{+} \widetilde{a}_{j_{N}}\right)^{\Delta J 1 / 2}\right\| \alpha_{i} J_{i} T_{i}\right\rangle & \\
= & (-)^{j_{N}+j_{\Lambda}-\Delta J} U\left(J_{i} j_{N} J_{f} j_{\Lambda}, J_{c} \Delta J\right) \\
& \times\left\langle\alpha_{c} J_{c} T_{f}\left\|\widetilde{a}_{j_{N}}\right\| \alpha_{i} J_{i} T_{i}\right\rangle .
\end{aligned}
$$

Here, $\alpha_{c}$ denotes a specific core state, $U$ is a unitary Racah coefficient for the recoupling of three angular momenta, $\Delta J$ is the angular momentum transfer, and the isospin transfer is $1 / 2$.

The radial part of the transition density is given by products of the $\Lambda$ and nucleon radial wave functions. Also, an overall isospin Clebsch-Gordan coefficient factors out of Eq. (2) leaving a reduced matrix element in isospace. Finally, one is left with a sum over products of radial integrals and OBDME for each permitted angular momentum transfer $\Delta J$. To see the consequences of the spin-flip characteristics of the reaction used to produce the hypernuclear states, it is useful to change the coupling from $\left(j_{N} j_{\Lambda}\right) \Delta J$ to $\left(l_{N} l_{\Lambda}\right) \Delta L \Delta S \Delta J$. For $\left(K^{-}, \pi^{-}\right)$ reactions near $800 \mathrm{MeV} / \mathrm{c}$ and $\left(\pi^{+}, K^{+}\right)$reaction at 1.04 $\mathrm{GeV} / \mathrm{c}, \Delta S=0$ dominates. On the other hand, for $\left(e, e^{\prime} K^{+}\right)$reactions $\Delta S=1$ dominates (especially for the favored high $\Delta J$ ); see the appendix of Millener (2012) for a discussion of the combinations of OBDME that govern the various production reactions.

As Eq. (3) shows, the OBDME is proportional to the spectroscopic amplitude for the removal of the struck nucleon from the target. This leads to the intuitive, and important result in the weak-coupling limit, that the total strength for forming the states in a weakcoupling multiplet (summing over $J_{f} j_{\Lambda}$, with $j_{\Lambda}$ denoting the members of a $\Lambda$ spin-orbit doublet) is proportional to the pickup spectroscopic factor, $\sum_{j_{N}} C^{2} S_{j_{N}}(c)$, from the target. Here, the isospin Clebsch-Gordan coefficient $C$ is obtained by changing the order of coupling in the overall isospin Clebsch-Gordan coefficient and $S_{j_{N}}(c)=<i\left\|a_{j_{N}}^{+}\right\| c>^{2}$. Failure to resolve the states in a multiplet strongly limits the information that can be obtained on the spin-dependence of the $\Lambda N$ interaction and underlines why high-resolution $\gamma$-ray detection is so important.

Distortions of the incident and exit waves generally do not change the shape of the angular distributions, but can reduce the reaction amplitudes by up to an order of magnitude [see Table 2 of Millener (1990)]. The factorized two-body amplitude must be averaged over the Fermi momentum of the participating nucleons in the medium. This can reduce the cross section typically by 
$10-20 \%$. Finally, the DWIA approximation assumes that the reaction amplitude can be expressed by a twobody on-shell $t$-matrix. Corrections to this approximation, and reaction processes that include instantaneous interactions with more than one nucleon, are expected to be small.

\section{Continuum excitations}

In many situations the $\Lambda$ is produced in unbound, continuum states. This especially occurs in high momentum transfer reactions, but even in the $\left(K^{-}, \pi^{-}\right)$substitutional reaction the $\Lambda$ can be unbound, as the $\Lambda$ nucleus well depth is approximately half of that of the nucleon-nucleus well depth. In hypernuclear production, this leads to the creation of a continuum background of excitations above the $\Lambda$-nucleus threshold. The continuum is sometimes discussed in terms of a QF reaction. In this model, the QF continuum spectrum is obtained by calculating the statistical density of states for the reaction on a single-particle nuclear state which produces an unbound $\Lambda$ recoiling under the influence of a $\Lambda$-nucleus potential. Calculations of the spectrum can be undertaken in a Fermi-gas model, so that the shape of the spectrum is determined by kinematics and the $\Lambda$-nucleus well depth (Dalitz and Gal, 1976). Applying this analysis to the continuum data of several medium-mass hypernuclei, a $\Lambda$-nucleus well depth of $\approx 30 \mathrm{MeV}$ is extracted.

On the other hand, contributions to the continuum spectrum should also include nuclear structure information. Inclusion of nuclear structure can be treated by several methods (Kishimoto, 1986, Motoba et al. 1988), the most common is the continuum shell model (Halderson, 1988), where the QF and resonant behavior are simultaneously calculated. The general features of continuum production are best observed by comparing the spectra from various reactions (Itonaga, Motoba, and Bandō, 1990). Above the continuum threshold, decay widths and the density of states increase rapidly. These appear as a rising, rather featureless background, with perhaps a few broad structures lying near threshold. When modified by final state interactions (Watson, 1952), the QF process can be applied to the extraction of the hyperon-nucleus interaction from the shape of the continuum spectrum near threshold.

\section{E. The nuclear Auger effect}

From previous arguments, a hypernucleus can be modeled as a set of single-particle nucleon holes and $\Lambda$ states. A reaction can place a $\Lambda$ particle in any of the bound or unbound levels of the nucleus, from which it may escape the nuclear potential well, cascade downward in energy, or become trapped in an isomeric level (Likar, Rosina, and Povh, 1986). A bound $\Lambda$ eventually reaches the ground state from which it weakly decays, see Fig. 2. The energy released in these transitions is removed either by $\gamma$ rays, or by Auger neutron (or perhaps proton) emission since nucleon emission thresholds can be lower than the $\Lambda$ emission threshold. However, nucleon emission can also occur from unbound $\Lambda$ states. Thus the final hypernuclear species may differ from the one initially produced. Indeed, the hypernuclear system may fragment, producing a residual hypernucleus much lower in mass. Consequently, hypernuclei can be studied not only in production, where the reaction is constrained by a few measured particles which completely determine the residual system, but also in decay, when the production process may be ill-determined but measurement of the decay products is sufficient to determine a specific hypernucleus. Therefore, unless some additional information is available, just measuring energies of $\gamma$-ray transitions is generally not sufficient to identify a hypernucleus or, moreover, the levels involved in the hypernuclear transition.

\section{F. Strangeness production: reactions and experimental techniques}

1. The $\left(K_{\text {stop }}^{-}, \pi^{-}\right)$reaction

Kaon capture at rest generally leads to $\Sigma$ rather than $\Lambda$ production. Approximately 5 times as many $\Sigma$ 's as $\Lambda$ 's are produced in $K^{-}$capture on carbon (Tamura et al., 1994). A strong $\Sigma$ QF production background is present in recent $\Lambda$ hypernuclear production experiments by the FINUDA Collaboration (Agnello et al., 2011b). The prominence of $\Sigma \pi$ relative to $\Lambda \pi$ final states in $K^{-}$ capture at rest is demonstrated in Table [II taken from $K_{\text {stop }}^{-}$reactions in bubble chambers (Velde-Wilquet et al. 1977). In this table, the $R$ factors are the branching fractions to a particular channel upon $K^{-}$capture, and the ratio $R_{n} / R_{p}$ is the ratio of captures on neutrons to captures on protons. The ratio $R_{m}$ is the branching ratio for capture on multinucleon clusters in the nucleus with

TABLE II Branching ratios (in \%) for hyperon production using stopped $K^{-}$(Velde-Wilquet et al., 1977).

\begin{tabular}{lccccc}
\hline \hline Ratio & $\mathrm{H}$ & $\mathrm{D}$ & $\mathrm{He}$ & $\mathrm{C}$ & $\mathrm{Ne}$ \\
\hline$R\left(\Lambda \pi^{0}\right)$ & 4.9 & 5. & 6.2 & 4.4 & 3.4 \\
$R\left(\Sigma^{+} \pi^{-}\right)$ & 14.9 & 30. & 37.3 & 37.7 & 37.7 \\
$R\left(\Sigma^{-} \pi^{+}\right)$ & 34.9 & 22. & 10.9 & 16.8 & 20.4 \\
$R\left(\Sigma^{0} \pi^{0}\right)$ & 21.4 & 23. & 21.2 & 25.7 & 27.6 \\
$R\left(\Lambda \pi^{-}\right)$ & 9.7 & 10. & 12.6 & 8.7 & 6.7 \\
$R\left(\Sigma^{0} \pi^{-}\right)$ & 7.1 & 5. & 5.9 & 3.3 & 2.1 \\
$R\left(\Sigma^{-} \pi^{0}\right)$ & 7.1 & 5. & 5.9 & 3.3 & 2.1 \\
$R_{n} / R_{p}$ & 0.31 & 0.25 & 0.32 & 0.18 & 0.12 \\
$R_{m}$ & & 0.01 & 0.16 & 0.19 & 0.23 \\
\hline \hline
\end{tabular}


no emitted pions, reaching values about $20 \%$ in nuclei beyond carbon. Of the several possible two-nucleon absorption channels, $\Sigma^{-} p$ pairs emitted in $K^{-}$capture at rest on $p$-shell nuclear targets have been observed at rates $\sim 4 \%$ (Agnello et al. , 2015). The table shows clearly a reversal of the ratio $R\left(\Sigma^{-} \pi^{+}\right) / R\left(\Sigma^{+} \pi^{-}\right)$when going from capture on hydrogen to capture on nuclear targets. This reflects the proximity of the $\Lambda(1405)$ subthreshold resonance which is more readily accessed kinematically in $K^{-}$reactions on nuclei, as studied recently in capture at rest experiments on $p$-shell nuclear targets Agnello et al. 2011a).

The $\left(K_{\text {stop }}^{-}, \pi^{-}\right)$reaction proceeds when a kaon is absorbed from an atomic orbit into the nucleus (Hüfner, Lee, and Weidenmüller, 1974). X-ray measurements of kaon absorption on ${ }^{12} \mathrm{C}$ (Wiegand and Mack, 1967) indicate that $20 \%$ of all the kaons are captured from $d$ orbits, while the remaining $80 \%$ are believed to be captured from low angular momentum, $l_{K}=0$ or 1 , and large $n_{K}$ states. Kaon absorption at rest provides momentum transfer approximately equal to the Fermi momentum of a bound $\Lambda$, and for a carbon target angular-momentum transfers $J \leq 4$ are possible. Since the stopped reaction has higher momentum transfer than the in-flight reaction, it is much less selective. In comparison, the QF process is stronger for stopped kaons than in flight, so that it becomes difficult to resolve states near $B_{\Lambda}=0$ due to QF background. Therefore the effectiveness of the stopped kaon reaction, particularly for the higher energy levels, is limited, even with improved energy resolution.

The $\left(K_{\text {stop }}^{-}, \pi^{-}\right)$reaction was extensively used to produce hypernuclei before separated kaon beam lines were available. During capture, a $\Lambda$ hyperon is produced by the reaction, $K^{-}+n \rightarrow \Lambda+\pi^{-}$. In the first counter experiment of this type at the CERN PS (Faessler et al. 1973), a kaon beam was brought to rest in a carbon target, and following the absorption of the kaon, a ${ }_{\Lambda}^{12} \mathrm{C}$ hypernucleus was formed and identified by the emission of the $\pi^{-}$. Two broad peaks were observed in the pion spectrum, one with $B_{\Lambda}=11 \pm 1 \mathrm{MeV}$ and the other with $B_{\Lambda}=0 \pm 1 \mathrm{MeV}$. The widths were dominated by the experimental resolution, $6 \pm 1 \mathrm{MeV}$, and the two peaks were subsequently identified as excitations with the $\Lambda$ residing in the $s$ and $p$ shells. Formation rates for these states were estimated as $(2 \pm 1) \times 10^{-4}$, and $(3 \pm 1) \times 10^{-4}$ per stopped kaon, respectively. In another $K_{\text {stop }}^{-}$experiment, the ${ }^{12} \mathrm{C}\left(K_{\text {stop }}^{-}, \pi^{-}\right){ }_{\Lambda}^{12} \mathrm{C}$ reaction was observed (Tamura et al., 1994), with rates per stopped kaon for the formation of these $s$-shell and $p$-shell states given by $(0.98 \pm 0.12) \times 10^{-3}$ and $(2.3 \pm 0.3) \times 10^{-3}$, respectively. These formation probabilities were a factor of 3 larger than those calculated by Gal and Klieb (1986) and a factor of 8 larger than the Matsuyama-Yazaki values (Matsuyama and Yazaki, 1988). However, the relative strength of the two peaks was found to be in better agreement with theory.

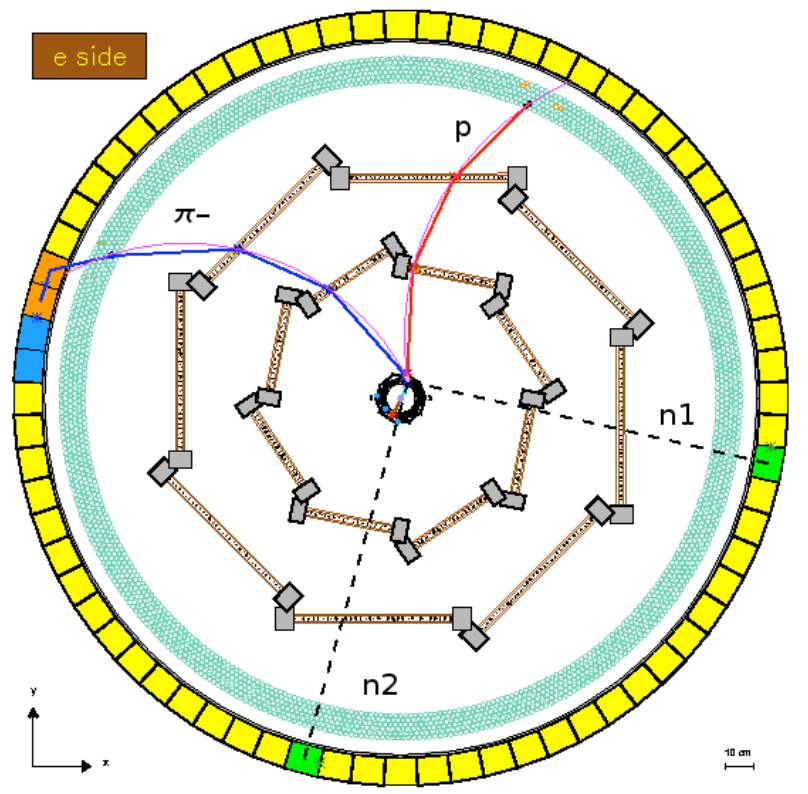

FIG. 3 Illustration of a $\Lambda n p \rightarrow n n p$ event observed by FINUDA on a ${ }^{7} \mathrm{Li}$ target. The $\pi^{-}$track arises from the formation of ${ }_{\Lambda}^{7} \mathrm{Li}$. Adapted from Agnello et al., 2012b.

More recently, the FINUDA Collaboration at the DA $\Phi$ NE $e^{+} e^{-}$colliding beam machine in Frascati reported stopped $K^{-}$formation rates on several $p$-shell targets from ${ }^{7} \mathrm{Li}$ to ${ }^{16} \mathrm{O}$ (Agnello et al. 2005b, 2011b). More hypernuclear levels in ${ }_{\Lambda}^{12} \mathrm{C}$ than the two main peaks seen in the earlier experiments were observed, with rates consistent for these two peaks with the earlier reports. These $p$-shell hypernuclear formation rates were then used in a theoretical study of the in-medium modification of the $\bar{K} N$ interaction, as derived within a coupled-channel chiral model, concluding that the $\left(K_{\text {stop }}^{-}, \pi^{-}\right)$reaction can be used to better determine the $K^{-}$-nuclear optical potential depth (Cieplý et al., 2011). FINUDA's special niche in hypernuclear physics was its remarkable performance connecting together production and decay of light $\Lambda$ hypernuclei. This will become clear in Sec. II.B.

FINUDA's capabilities are demonstrated in Fig. 3 by showing a complete kinematical reconstruction of a threenucleon final state in one of two ${ }_{\Lambda}^{7} \mathrm{Li} \rightarrow{ }^{4} \mathrm{He}+n+n+p$ decay events observed at DA $\Phi$ NE (Agnello et al., 2012b) following stopped- $K^{-}$formation of ${ }_{\Lambda}^{7} \mathrm{Li}$ on a ${ }^{7} \mathrm{Li}$ target, with a production $\pi^{-}$track clearly visible. In another recent example, by correlating $\pi^{+}$mesons from the $\left(K_{\text {stop }}^{-}, \pi^{+}\right)$production reaction on a ${ }^{6} \mathrm{Li}$ target with weak-decay $\pi^{-}$mesons, three events were observed that were interpreted as production of the neutron-rich exotic hypernucleus ${ }_{\Lambda}^{6} \mathrm{H}$ that subsequently undergoes a twobody ${ }_{\Lambda}^{6} \mathrm{H} \rightarrow \pi^{-}+{ }^{6} \mathrm{He}$ weak decay (Agnello et al., 2012a, 2012c). 
TABLE III $\Lambda$ hypernuclear formation rates in $K^{-}$capture at rest on ${ }^{12} \mathrm{C}$, in units of $10^{-3}$ per stopped $K^{-}$.

\begin{tabular}{|c|c|c|c|}
\hline & Reference & $R\left(s_{1 / 2}\right) \times 10^{3}$ & $R\left(p_{3 / 2}+p_{1 / 2}\right) \times 10^{3}$ \\
\hline${ }_{\Lambda}^{12} \mathrm{C}$ theory & Gal and Klieb (1986) & 0.33 & 0.96 \\
\hline${ }_{\Lambda}^{12} \mathrm{C}$ theory & Matsuyama and Yazaki (1988) & 0.12 & 0.59 \\
\hline${ }_{\Lambda}^{12} \mathrm{C}$ theory & Krejčiřík, Cieplý, and Gal $(2010)^{a}$ & $0.13-0.43$ & $0.43-1.27$ \\
\hline${ }_{\Lambda}^{12} \mathrm{C}$ experiment & Tamura et al. (1994) & $0.98 \pm 0.12$ & $2.3 \pm 0.3$ \\
\hline${ }_{\Lambda}^{12} \mathrm{~B}$ theory & Krejčiřík, Cieplý, and Gal $(2010)^{a}$ & $0.06-0.20$ & $0.20-0.64$ \\
\hline${ }_{\Lambda}^{12} \mathrm{~B}$ experiment & Ahmedet al. $(2003)^{\sqrt{b}}$ & $0.28 \pm 0.08$ & $0.35 \pm 0.09$ \\
\hline
\end{tabular}

a Depending on the $K^{-}$nuclear potential, from deep to shallow.

b Multiply by 2 to compare to ${ }_{\Lambda}^{12} \mathrm{C}$ production.

\section{The $\left(K_{\text {stop }}^{-}, \pi^{0}\right)$ reaction}

The $\left(K_{\text {stop }}^{-}, \pi^{0}\right)$ reaction is an example in which both strangeness and charge are exchanged. However, it is expected to have the same features as the $\left(K_{\text {stop }}^{-}, \pi^{-}\right)$reaction, although its cross section is reduced by the isospin ratio of $1 / 2$. This reaction produces hypernuclear species charge symmetric to those studied by the $\left(K^{-}, \pi^{-}\right)$and $\left(\pi^{+}, K^{+}\right)$reactions. In this reaction, the two photons from the $\pi^{0}$ decay can be used to identify and measure the energy of the outgoing $\pi^{0}$. Thus, not only do all the stopped $K^{-}$'s interact in the nuclear target but a thick target can be used without degrading the energy resolution because captured $K^{-}$'s have essentially zero momentum and the decay photons easily penetrate the target without significant energy degradation.

Comparison of the spectra of charge-symmetric hypernuclei provides information that could be helpful to extract the isospin asymmetry of the fundamental $\Lambda N$ interaction. This has been studied to some extent in the ground states of $s$ - and $p$-shell mirror hypernuclear pairs, but aside from binding energies, few comparative data are available (Gibson and Hungerford, 1995). However, in addition to charge asymmetry in the fundamental $\Lambda N$ interaction, Coulomb effects can lead to energy differences between charge-symmetric hypernuclei, in part because the added $\Lambda$ compresses its nuclear core, thereby increasing its Coulomb energy (Hiyama et al., 1999). Therefore, a careful study of the spectra of several charge symmetric pairs is needed to extract both the Coulomb and charge asymmetry effects for the excited, as well as the hypernuclear ground states (Gal, 2015).

In an experiment at BNL (Ahmed et al. 2003 ), $\pi^{0}$ 's were detected by observing the opening angle of the decay photons from the $\pi^{0}$ using a neutral meson spectrometer (NMS). The NMS (Morris et al., 1989) was a large acceptance photon detector which measured the total energy of a $\pi^{0}$. It consisted of two arrays of 60 CsI crystals each fronted by a set of bismuth germanate (BGO) converter and wire chamber tracking planes. The CsI crystals provide the photon calorimetry to determine the relative energy difference between the decay photons, while the BGO and wire chambers determine the loca- tion of the photon conversion. A dispersed $K^{-}$beam with a nominal momentum of $690 \mathrm{MeV} / \mathrm{c}$ was brought to rest in a set of 4 natural graphite targets after it traversed a wedge-shaped, brass degrader of central thickness $\approx 141 \mathrm{~mm}$. The degrader compensated for the beam dispersion $(\approx 1.2 \mathrm{MeV} / \mathrm{c}$ per $\mathrm{cm})$. The energy resolution was $2.2 \mathrm{MeV}$ (FWHM) which was primarily attributed to problems associated with maintaining energy calibrations over the long period of data acquisition.

In this experiment, the hypernuclear ground-state formation probability was found to be $(0.28 \pm 0.08) \times 10^{-3}$ and that for the $p$-shell states near the $\Lambda$ emisson threshold was $(0.35 \pm 0.09) \times 10^{-3}$. This is compared in Table III to theoretical and experimental values for the $\left(K_{\text {stop }}^{-}, \pi^{-}\right)$reaction, that should occur twice as often assuming good isospin symmetry. The quoted errors are statistical, but because of the difficulty in extracting the yield from background the systematic error is somewhat larger for the $p$ shell (about 15\%). Following kinematical corrections to isospin conservation, the formation probability to the ground state is lower than the previous experimental value for ${ }_{\Lambda}^{12} \mathrm{C}$ formation. However, this result still remains higher than the theoretical calculations for the ground state (Gal and Klieb, 1986; Matsuyama and Yazaki, 1988; Cieplý et al., 2001, 2003; Krejčiřík, Cieplý and Gal, 2010).

\section{The in-flight $\left(K^{-}, \pi^{-}\right)$reaction}

Although hypernuclear spectroscopy was initially studied with stopped kaon beams, the in-flight $\left(K^{-}, \pi^{-}\right)$reaction was introduced to take advantage of intense sources of secondary beams and the adoption of modern electronic counting to the readout of magnetic spectrometers. The in-flight reaction has several advantages as described below.

The in-flight $\left(K^{-}, \pi^{-}\right)$reaction was first used at CERN (Bonazzola et al., 1974, Brückner et al., 1975) and then at BNL (Chrien et al. 1979) for incident kaon momenta in the range of 700 to $900 \mathrm{MeV} / \mathrm{c}$ where the elementary cross section has a maximum [see Fig. 6 of Hashimoto and Tamura (2006)]. Another important fea- 


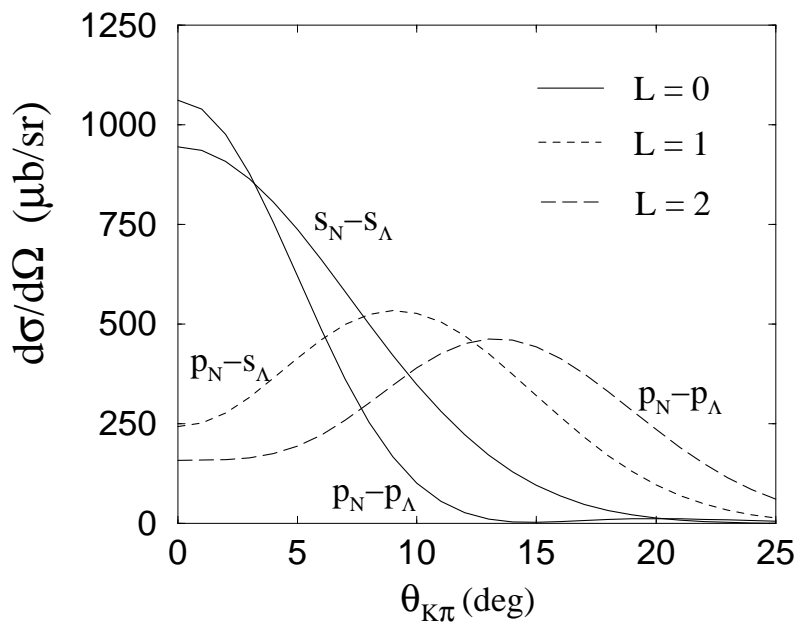

FIG. 4 Angular distributions for the $\left(K^{-}, \pi^{-}\right)$reaction for pure single-particle transitions on ${ }^{16} \mathrm{O}$ at $p_{K}=900 \mathrm{MeV} / \mathrm{c}$. $\Delta L$ is the orbital angular momentum transfer for the indicated transitions. From Ukai et al., 2008.

ture of the elementary reaction at these momenta is that the spin-flip amplitudes are small. As Fig. 11 shows, the momentum transfer to the hypernucleus is still small in the forward direction, favoring no transfer of orbital (or spin) angular momentum. In this case, the spectra of light hypernuclei exhibit peaks when a $\Lambda$ replaces a neutron without changing the quantum numbers of the single-particle orbit. This is illustrated in Fig. 4 for pure single-particle transitions on ${ }^{16} \mathrm{O}$ at $p_{K}=800 \mathrm{MeV} / \mathrm{c}$. The resulting $\Lambda$ hypernuclear states are called "substitutional states" (populated via "recoilless" transitions). The strong nuclear absorption of the incident $K^{-}$and exiting $\pi^{-}$limits penetration into the nucleus and favors transitions with surface-peaked transition densities (generally, between nodeless orbits).

One of the early investigations used the spin splitting of states in ${ }_{\Lambda}^{16} \mathrm{O}$ to obtain a value for the $\Lambda$-nucleus spin orbit interaction. In Figure 5, the splitting of the two $p_{\Lambda}$ states $\left(0_{1}^{+}\right.$and $\left.0_{2}^{+}\right)$, observed in the ${ }^{16} \mathrm{O}\left(K^{-}, \pi^{-}\right){ }_{\Lambda}^{16} \mathrm{O}$ reaction spectrum, shows that the energy difference between the states obtained when replacing a $p_{1 / 2}$ or $p_{3 / 2}$ neutron by a $\Lambda$ is essentially the same as the energy splitting of the hole states in ${ }^{15} \mathrm{O}(6.18 \mathrm{MeV})$. This indicates that the effective $\Lambda N$ spin-orbit splitting is small (Povh, 1980), a conclusion that remains valid when the residual $\Lambda N$ interaction is taken into account (Bouyssy, 1980). A small effective $\Lambda N$ spin-orbit potential was also confirmed in the analysis of the angular distribution of the $p_{\Lambda}$ substitutional peak based on the ${ }^{12} \mathrm{C}$ ground state observed in the ${ }^{13} \mathrm{C}\left(K^{-}, \pi^{-}\right){ }_{\Lambda}^{13} \mathrm{C}$ reaction spectrum. In this experiment (May et al. 1981$)$, the $p_{1 / 2 \Lambda}$ state is formed via a $\Delta L=0$ transition near $0^{\circ}$ while the $p_{3 / 2 \Lambda}$ state is formed via a $\Delta L=2$ transition near $15^{\circ}$ (see Fig. 4). Therefore, by measuring a shift of $0.36 \pm 0.3 \mathrm{MeV}$ in the

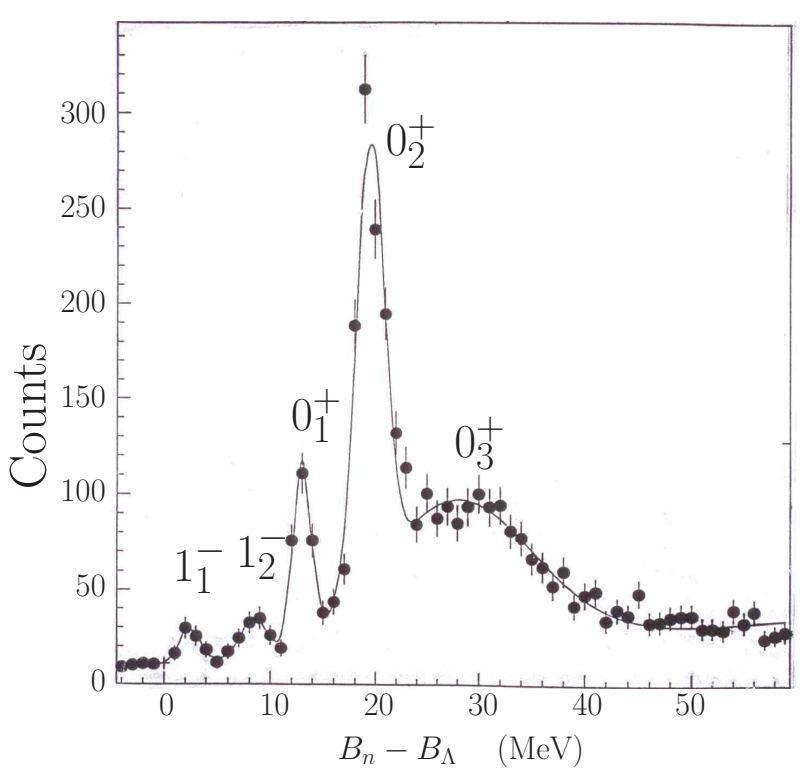

FIG. 5 Spectrum for the $\left(K^{-}, \pi^{-}\right)$reaction on ${ }^{16} \mathrm{O}$ at incident momentum $p_{K}=715 \mathrm{MeV} / \mathrm{c}$ near $0^{\circ}$ (Brückner et al. 1978). The $1^{-}$states are $s_{\Lambda}$ states based on the $p_{1 / 2}^{-1}$ and $p_{3 / 2}^{-1}$ hole states of ${ }^{15} \mathrm{O}$. The $0_{1,2}^{+}$states are $p_{\Lambda}$ substitutional states based on the same core states, while the $0_{3}^{+}$state is based on the broad $0 s$-hole strength in ${ }^{15} \mathrm{O}$. For ${ }^{16} \mathrm{O}, B_{n}=15.66 \mathrm{MeV}$, so that $B_{\Lambda} \sim 13 \mathrm{MeV}$ for the $1_{1}^{-}$state. The refit of the data is due to D. H. Davis and D. N. Tovee. Courtesy of D. H. Davis.

excitation of the substitutional peak between $0^{\circ}$ and $15^{\circ}$, the $\Lambda$ spin-orbit coupling was shown to be small (Auerbach et al. 1981, 1983). Finally, the $\Lambda$ spin-orbit splitting in ${ }_{\Lambda}^{13} \mathrm{C}$ was found to be very small by observing two $\gamma$ rays of energy $\approx 11 \mathrm{MeV}$, taken to be $p_{j \Lambda} \rightarrow s_{1 / 2 \Lambda}$ $E 1$ transitions correlated with two constituent states in this substitutional peak, and found to be split by $152 \pm 54$ (stat) \pm 36 (syst) keV (Ajimura et al. 2001, Kohri et al. 2002).

After the initial success in applying the $\left(K^{-}, \pi^{-}\right)$reaction to $\Lambda$ hypernuclei, an attempt was made to look for bound $\Sigma$ states using the same reaction (Bertini et al. 1980, 1984, 1985). Although it was expected that such structures would have a large width due to the strong conversion $\Sigma N \rightarrow \Lambda N$, this research remained in a confused state for a number of years, limited by the low statistics of the experiments which perhaps also encouraged theoretical speculations. Experimentally, a number of light $\Sigma$-nuclear systems were investigated, particularly for $s$ - and $p$-shell $\Sigma$ nuclear systems. Attempts were made to use lower incident kaon momentum to reduce the QF component in the reaction, and to enhance substitutional-state production. All these investigations indicated some reaction strength below the $\Sigma$ emission threshold, but the interpretation of the observed structure was limited by statistical fluctuations (Dover, Millener, and Gal, 1989). 

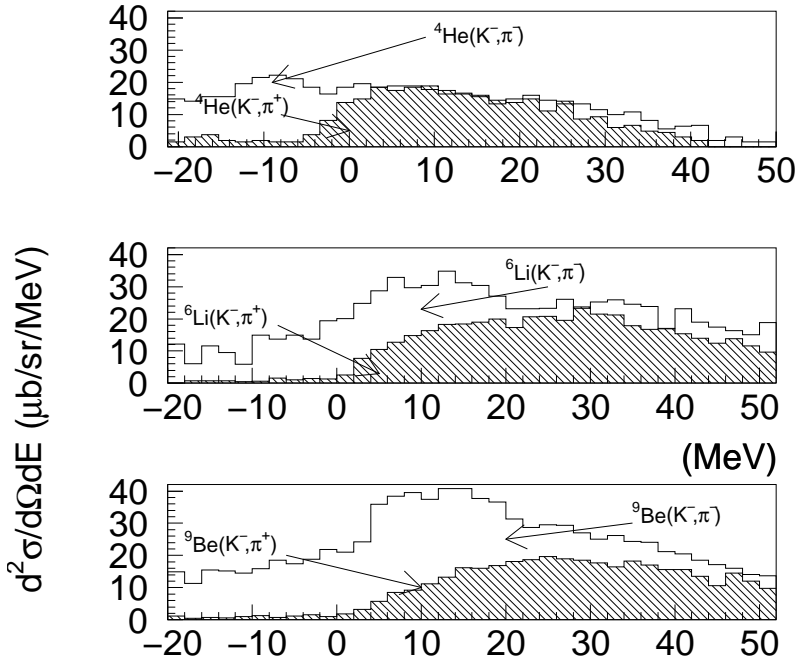

FIG. 6 Experimental data from various targets showing the progressive changes in the $\Sigma$-nucleus interaction for both $\left(K^{-}, \pi^{-}\right)$and $\left(K^{-}, \pi^{+}\right)$reactions. From Bart et al., 1999.

Two high-statistics experiments were eventually completed, one (Nagae et al. 1998) on a ${ }^{4} \mathrm{He}$ target and one (Bart et al. 1999) on a series of $p$-shell nuclei. The result provided a consistent picture for $\Sigma$ nuclear interactions in light nuclear systems. A significant dependence on isospin was found by observing production differences in the spectra from $\left(K^{-}, \pi^{-}\right)$and $\left(K^{-}, \pi^{+}\right)$reactions. This is shown in Fig. 6 where one sees a progressive shift of the enhancement below threshold to higher energies and a broadening of its width. In the specific case of ${ }^{4} \mathrm{He}$, a broad bound state having a binding energy of $\approx 4.4 \mathrm{MeV}$ with a width of $\approx 7.0 \mathrm{MeV}$ was observed. Note that this state must have isospin $1 / 2$, as it is seen only in the $\left(K^{-}, \pi^{-}\right)$reaction.

The presence of isospin dependence suggests a strong "Lane" term in the potential which would have a $1 / A$ dependence, reducing the possibility of $\Sigma$ hypernuclear states of any width for $A>4$. In particular for the $A=4$ system, theoretical analysis has shown that the effective $\Sigma$ nucleus potential has a small attractive pocket near the nuclear surface, and a strong repulsive core which decreases exponentially as the nuclear radius increases. A bound $\Sigma$ could reside in this well and, as the nuclear surface has lower density, the conversion width of the $\Sigma$ is smaller allowing a broad state to form Harada et al. 1990).

More recently a study of $\Sigma$-nuclear systems was completed using the $\left(\pi^{-}, K^{+}\right)$reaction in flight on several targets (e.g., C, Si). This reaction converts in one step a target proton to a $\Sigma^{-}$hyperon. While the resulting spectra show a non-vanishing residual strength below the $\Sigma$ hypernuclear threshold, no evidence for bound states was found. Indeed, when analyzed in DWIA, the spectra are reproduced only by using a strongly repulsive $\Sigma$-nucleus potential (Saha et al. 2004$)$. The $\left(\pi^{-}, K^{+}\right)$reaction was also studied near the $\Lambda$ hypernuclear threshold on a ${ }^{10} \mathrm{~B}$ target, searching for bound states in the neutron-rich ${ }_{\Lambda}^{10} \mathrm{Li}$ hypernucleus (Saha et al. 2005$)$. Although no clear peaks could be resolved in the $\Lambda$ bound region, the size of the deduced cross section is consistent with formation of ${ }_{\Lambda}^{10} \mathrm{Li}$ through a $\Sigma^{-}$admixture of probability $\approx 0.1 \%$ induced by $\Sigma^{-} p \leftrightarrow \Lambda n$ coupling (Harada, Umeya, and Hirabayashi, 2009). Very recently J-PARC experiment E10, using the $\left(\pi^{-}, K^{+}\right)$reaction on a ${ }^{6} \mathrm{Li}$ target, did not observe any significant strength in the ${ }_{\Lambda}^{6} \mathrm{H}$ bound region (Sugimura et al. 2014), indicating perhaps a weaker appropriate $\Sigma^{-}$admixture than in ${ }_{\Lambda}^{10} \mathrm{Li}$. This leaves open the question of whether or not the exotic neutron-rich hypernucleus ${ }_{\Lambda}^{6} \mathrm{H}$ is particle stable as indicated by the FINUDA experiment using a $\left(K_{\text {stop }}^{-}, \pi^{+}\right)$production reaction (Agnello et al. 2012a c); see the discussion at the end of Sec. I.F.1 and the recent calculations by Gal and Millener (2013) and Hiyama et al. (2013).

\section{The $\left(\pi^{+}, K^{+}\right)$reaction}

The study of hypernuclear spectra using the $\left(\pi^{+}, K^{+}\right)$ reaction (Dover, Ludeking, and Walker, 1980; Thiessen et al., 1980) was first explored at the BNL-AGS in a series of investigations providing spectra across a wide range of hypernuclei. Typical energy resolution of 3-4 MeV was obtained (Milner et al., 1985; Pile et al., 1991). The reaction was then explored in detail at KEK with a dedicated beamline and a high resolution spectrometer, SKS (Fukuda et al. . 1995), specifically built to detect the reaction kaons. Using this system, the resolution improved to about $2 \mathrm{MeV}$ (Nagae, 2001).

The elementary reaction $n\left(\pi^{+}, K^{+}\right) \Lambda$ peaks at an incident pion momentum near $1.05 \mathrm{GeV} / \mathrm{c}$, as shown in Fig. 7. and all $\left(\pi^{+}, K^{+}\right)$experiments have been performed at this incident momentum. The outgoing $K^{+}$ has a momentum of $\approx 0.7 \mathrm{GeV} / \mathrm{c}$ and the momentum and angular-momentum transfer to the $\Lambda$ is substantial. The $\left(\pi^{+}, K^{+}\right)$reaction then preferentially populates spin-stretched states with an angular-momentum transfer $\Delta L=l_{n}+l_{\Lambda}$. For nodeless orbitals, the momentum dependence (form factor) of the transition density (product of radial wave functions) is given by $y^{\Delta L / 2} e^{-y}$ with $y=(b q / 2)^{2}$, where $q$ is the 3 -momentum transfer and $b$ is the harmonic oscillator parameter $\left(b^{2}=41.5 / \hbar \omega\right.$, $\left.\hbar \omega=45 A^{-1 / 3}-25 A^{-2 / 3}\right)$. The maximum of the form factor occurs for $y=\Delta L / 2$. For light hypernuclei and transitions to inner $\Lambda$ orbitals in heavier nuclei, the momentum transfer $q$ is generally over $300 \mathrm{MeV} / \mathrm{c}$ which is well past the peak in the form factor and cross sections are small. However, the $\left(\pi^{+}, K^{+}\right)$reaction becomes more effective in producing states with large $l_{\Lambda}$ in heavier hypernuclei due to the increasing spin of the valence neutron orbital involved in the reaction. Indeed, in Fig. 8 , the full spectrum of nodeless, bound $\Lambda$ orbitals is clearly 


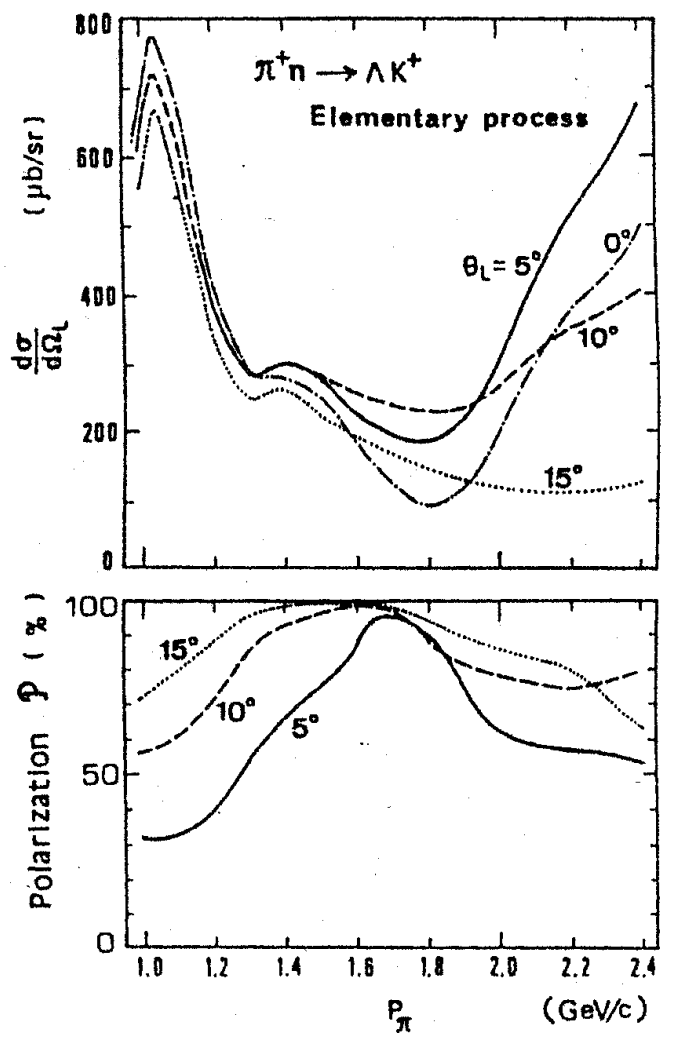

FIG. 7 The elementary $n\left(\pi^{+}, K^{+}\right) \Lambda$ reaction and the polarization of the $\Lambda$ as a function of the $\pi$ incident momentum. From Bandō et al., 1989.

evident for the ${ }_{\Lambda}^{89} \mathrm{Y}$ hypernucleus (Hotchi et al., 2001). The main part of the cross section arises from associated production on a $g_{9 / 2}$ neutron, while the origin of possible fine structure in the peaks is open to interpretation $(\mathrm{Mo}-$ toba et al. 2008). The $\Delta L=7$ transition dominating the $f_{\Lambda}$ peak is well matched in the sense that the peak of the form factor occurs for $q \sim 345 \mathrm{MeV} / \mathrm{c}$ and closely matches the momentum transfer to the hypernucleus. In general, $\left(\pi^{+}, K^{+}\right)$cross sections are found to be roughly a factor of 100 below those in the $\left(K^{-}, \pi^{-}\right)$reactions (different final states are populated) but, in terms of running time, the decrease in cross section can be more than compensated by the increased intensity of pion beams. Because the momentum transfer is high, the cross section falls rapidly with angle and the angular distribution is not a good indicator of the angular momentum transfer.

In contrast to low momentum kaon induced reactions, the $\Lambda$ recoil in the $\left(\pi^{+}, K^{+}\right)$reaction has substantial polarization at finite forward angles. This polarization is due to a combination of the difference the near- and far side absorption of the incident pion, and the spin dependence of the elementary interaction. With the exception that polarization creates specific spin states in the hy-

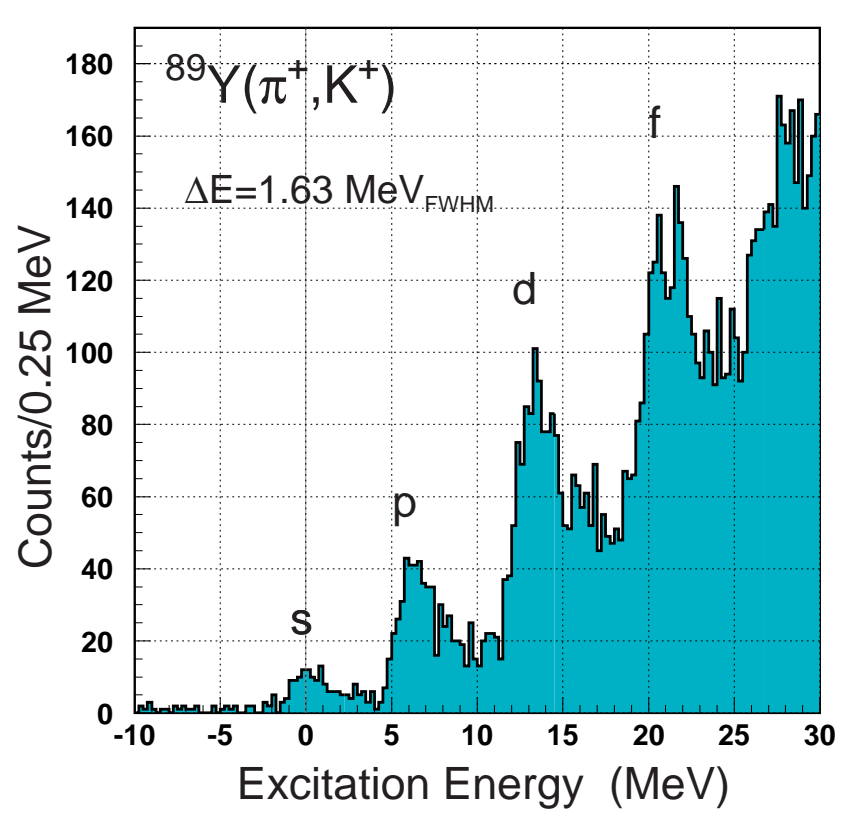

FIG. 8 The hypernuclear spectrum of ${ }_{\Lambda}^{89} \mathrm{Y}$ from KEK E369 showing the major $\Lambda$ shell structure. From Hotchi et al., 2001.

pernucleus, polarization in the $\left(\pi^{+}, K^{+}\right)$reaction has not been experimentally used in spectroscopic studies, (e.g., angular correlations), as these experiments require coincidence measurements at angles where the production rate is low.

The $\left(\pi^{+}, K^{+}\right)$reaction has so far been the most productive spectroscopic reaction across a wide range of nuclei. However, targets are large (e.g., several $\mathrm{cm}^{2}$ in area and $\approx \mathrm{gm} / \mathrm{cm}^{2}$ thick) which is a factor in limiting the energy resolution. The choice of target is a factor in the selectivity of the reaction. As noted earlier, cross sections are proportional to the neutron pickup spectroscopic factor in the weak-coupling limit. This means that ideally one should choose a target with a full shell of a high- $j$ neutron orbit close to the Fermi surface. At A 90, this would mean a ${ }^{90} \mathrm{Zr}$ target but ${ }^{89} \mathrm{Y}$ has the advantage that it is a monotope; the ${ }^{88} \mathrm{Y}$ core nucleus has a $4^{-}$ground state and a low-lying $5^{-}$state (at $232 \mathrm{keV}$ ) that are both fed by $g_{9 / 2}$ neutron removal and a small correction must be made to the extracted $B_{\Lambda}$ values (Hasegawa et al. 1996).

The $\left(\pi^{+}, K^{+}\right)$reaction provides a textbook example of the single-particle shell structure of hypernuclei, with Fig. 8 showing the prime example. In Sec. I.F.6, we collect together the $\Lambda$ single-particle energies in terms of $B_{\Lambda}$ values extracted from $\left(\pi^{+}, K^{+}\right),\left(e, e^{\prime} K^{+}\right),\left(K^{-}, \pi^{-}\right)$, and emulsion studies. Most of the values come from three $\left(\pi^{+}, K^{+}\right)$experiments at KEK, namely E140a (Hasegawa et al. 1996) (targets ${ }^{10} \mathrm{~B},{ }^{12} \mathrm{C},{ }^{28} \mathrm{Si},{ }^{89} \mathrm{Y},{ }^{139} \mathrm{La},{ }^{208} \mathrm{~Pb}$ ), E336 (Hashimoto et al., 1998; Hashimoto and Tamura, 2006) (targets ${ }^{7} \mathrm{Li},{ }^{9} \mathrm{Be},{ }^{12} \mathrm{C},{ }^{13} \mathrm{C},{ }^{16} \mathrm{O}$ ), and E369 (Hotchi et al. 2001) (targets ${ }^{12} \mathrm{C},{ }^{51} \mathrm{~V},{ }^{89} \mathrm{Y}$ ). All the targets are 
largely a single isotope, either because the natural target is a monotope, or nearly so, or because an enriched target was used $\left({ }^{7} \mathrm{Li},{ }^{10} \mathrm{~B},{ }^{13} \mathrm{C},{ }^{208} \mathrm{~Pb}\right)$. For the heavier targets $\left({ }^{51} \mathrm{~V},{ }^{89} \mathrm{Y},{ }^{139} \mathrm{La},{ }^{208} \mathrm{~Pb}\right)$, the aim is to identify peaks due to a series of $\Lambda$ orbitals based on holes in the nodeless $f_{7 / 2}, g_{9 / 2}, h_{11 / 2}$, and $i_{13 / 2}$ neutron shells. For the odd-mass targets there is fragmentation of the neutron pickup strength due to the presence of an odd proton, and this must be accounted for in the analysis. In addition, other filled neutron orbits can make substantial contributions to the cross sections, as can be seen from attempts to analyze the data for ${ }_{\Lambda}^{139} \mathrm{La}$ and ${ }_{\Lambda}^{208} \mathrm{~Pb}$ in Fig. 27 of Hashimoto and Tamura (2006) . We note that although plane-wave impulse approximation calculations seem to capture the essential physics (Bender, Shyam, and Lenske, 2010), DWIA calculations generally give in addition reliable estimates for the cross sections of states populated in the $\left(\pi^{+}, K^{+}\right)$reaction (Motoba et al., 1988; Millener, 1990; Motoba, Itonaga, and Yamamoto, 2010).

\section{The $\left(e, e^{\prime} K^{+}\right)$reaction}

Traditionally, hypernuclei were produced with secondary beams of kaons or pions. Because the $\left(K^{-}, \pi^{-}\right)$ reaction is exothermic, the 3 -momentum transfer to the $\Lambda$ hypernucleus can be chosen to be small. In the $\left(K^{-}, \pi^{-}\right)$ reaction, the cross section to substitutional states (i.e., states where the $\Lambda$ acquires the same shell quantum numbers as those of the neutron which it replaces) is relatively large. On the other hand, the $\left(\pi^{+}, K^{+}\right)$reaction has a 3momentum transfer comparable to the nuclear Fermi momentum, and the reaction preferentially populates states with high angular momentum transfers (Milner et al., 1985; Bandō and Motoba, 1986). Neither of these two reactions has significant spin-flip amplitude at forward angles, and consequently all spectra are dominated by transitions to non spin-flip states. Also, aside from early emulsion experiments, mesonic-reaction spectroscopy has generally provided hypernuclear spectra with energy resolutions $\approx 2 \mathrm{MeV}$. This is due to the intrinsic resolutions of secondary mesonic beamlines, and the target thicknesses required to obtain sufficient counting rates. However, one study did achieve a spectrum resolution of approximately $1.5 \mathrm{MeV}$ for the ${ }_{\Lambda}^{12} \mathrm{C}$ hypernucleus, using a thin target and devoting substantial time to data collection (Hotchi et al., 2001$)$. Another significant problem with the $\left(K^{-}, \pi^{-}\right)$and $\left(\pi^{+}, K^{+}\right)$reactions is how to fix the absolute scale of hypernuclear binding energies (no free-neutron target) and this requires normalization to a known ground-state binding energy, e.g. from emulsion data; see the discussion in Sec. I.F.6.

Electron beams, in comparison, have excellent spatial and energy resolutions, and the exchange of a photon can be accurately described by a first order perturbation calculation. In addition, electroproduction has been used for precision studies of nuclear structure so many experimental techniques are well established. Although previous electron accelerators had poor duty factors significantly impairing high singles-rate coincidence experiments, continuous-beam accelerators have now overcome this limitation. The cross section for nuclear kaon electroproduction is smaller than that for hypernuclear production by the $(\pi, K)$ reaction for example, but reaction rates can be compensated by increased beam intensity. Targets can be physically small and thin (10-100 mg $\mathrm{cm}^{-2}$ ), allowing studies of almost any isotope. However, a great advantage of the $\left(e, e^{\prime} K^{+}\right)$reaction is the potential to reach energy resolutions of a few hundred $\mathrm{keV}$ with reasonable counting rates at least up to mediumweight hypernuclei (Hungerford, 1994). Another great advantage is that the $\Lambda$ and $\Sigma^{0}$ peaks from the $\left(e, e^{\prime} K^{+}\right)$ reaction on hydrogen can be used to calibrate the hypernuclear binding-energy scale.

Furthermore, the $\left(e, e^{\prime} K^{+}\right)$reaction proceeds by the absorption of a spin-1 virtual photon which carries high spin-flip probability even at forward angles. The 3momentum transfer to a quasi-free $\Lambda$ is high (approximately $300 \mathrm{MeV} / \mathrm{c}$ at zero degrees for $1.5 \mathrm{GeV}$ incident photons), so the resulting reaction is expected to predominantly excite spin-flip transitions to spin-stretched states (Motoba, Sotona, and Itonaga, 1994). Recall that spin-flip states are not strongly excited in hadronic production, and the $\left(e, e^{\prime} K^{+}\right)$reaction acts on a proton rather than a neutron, creating proton-hole $\Lambda$-particle states which are charge symmetric to those studied with meson beams.

In electroproduction, the $\Lambda$ and $K^{+}$particles are created associatively via an interaction between a virtual photon and a bound proton, $p\left(\gamma, K^{+}\right) \Lambda$. The hypernucleus, ${ }_{\Lambda}^{A} \mathrm{Z}$, is formed by coupling the $\Lambda$ to the residual nuclear core ${ }^{(A-1)}(Z-1)$. In electroproduction, the energy and 3-momentum of the virtual photon are defined by $\omega=E_{e}-E_{e}^{\prime}$ and $\boldsymbol{q}=\boldsymbol{p}_{e}-\boldsymbol{p}_{e}^{\prime}$, respectively. The square of the four-momentum transfer of the electron is then given by $-Q^{2}=t=\omega^{2}-q^{2}$. As will be shown below, the number of (virtual) photons falls rapidly as the scattered electron angle increases (increasing $t$ ), and thus the distribution of (virtual) photons also peaks in the forward direction. In addition, the nuclear transition matrix element causes the cross section for hypernuclear production to fall rapidly as the angle between the reaction kaon and the (virtual) photon increases. Thus, experiments must be done within a small angular range around the direction of the incident electron. To accomplish this, the experimental geometry requires two spectrometer arms, one to detect the scattered electron and one to detect the kaon, both placed at extremely forward angles.

The electroproduction cross section can be expressed 
(Sotona and Frullani, 1994) by

$$
\begin{aligned}
\frac{d^{3} \sigma}{d E_{e}^{\prime} d \Omega_{e}^{\prime} d \Omega_{K}}= & \Gamma\left[\frac{d \sigma_{T}}{d \Omega_{K}}+\epsilon \frac{d \sigma_{L}}{d \Omega_{K}}+\epsilon \cos (2 \phi) \frac{d \sigma_{T T}}{d \Omega_{K}}\right. \\
& \left.+\cos (\phi) \sqrt{2 \epsilon(1+\epsilon)} \frac{d \sigma_{L T}}{d \Omega_{K}}\right]
\end{aligned}
$$

The factor $\Gamma$ is the virtual flux factor evaluated with electron kinematics in the lab frame, and $\phi$ is the angle measuring the out-of-plane production of the kaon with respect to the plane containing the beam and scattered electron. The factor $\Gamma$ has the form

$$
\Gamma=\frac{\alpha}{2 \pi^{2} Q^{2}}\left[\frac{E_{\gamma}}{1-\epsilon}\right] \frac{E_{e}^{\prime}}{E_{e}} .
$$

In the above equations, $E_{\gamma}=\omega, \alpha$ is the fine-structure constant and $\epsilon$ is the polarization factor

$$
\epsilon=\left[1+\frac{2|\boldsymbol{q}|^{2}}{Q^{2}} \tan ^{2}\left(\theta_{e} / 2\right)\right]^{-1}
$$

The label on each of the cross-section expressions $(T$, $L, T T$ and $L T$ ) represent transverse, longitudinal, polarization, and interference terms. For real photons of course, $Q^{2}=0$, so only the transverse cross section is non-vanishing, and for a very forward experimental geometry, the virtual photons are almost on the mass shell where $Q^{2}=\boldsymbol{p}_{\gamma}^{2}-E_{\gamma}^{2}=0$ so the cross section is completely dominated by the transverse component. Thus a good approximation replaces electroproduction cross section by the photoproduction cross section multiplied by a flux factor.

Experimentally, $\Gamma$ is integrated over the angular and momentum acceptances of the electron spectrometer. In order to maximize the cross section of the elementary $p\left(\gamma, K^{+}\right) \Lambda$ reaction, the photon energy is chosen to be $\approx(1.5-2.5) \mathrm{GeV}$. To maximize the elementary cross section, the virtual photon energy should be near $1.5 \mathrm{GeV}$, which determines the scattered electron energy, $E_{e^{\prime}}=$ $E_{e}-\omega$. Finally, to limit the production of a background of unwanted hyperons, the maximum choice for the beam energy should be as close to $1.8 \mathrm{GeV}$ as possible. The virtual flux factor peaks at zero degrees and falls rapidly as the scattering angle increases (Xu and Hungerford, 2003). A large percentage of the scattered electrons can be captured in even a small solid angle for scattering angles near zero degrees.

Compared to secondary beam experiments, the magnetic optics of the spectrometer systems in electroproduction experiments are less complicated because of the small beam spot $(\approx 100 \mu \mathrm{m})$, the $\leq 4^{\circ}$ electron scattering angle, and the small momentum value of the scattered electron. However, the disadvantage of this geometry is a high electron background rate from target bremsstrahlung, which ultimately limits the usable beam luminosity and drives the geometry away from in-plane scattering.
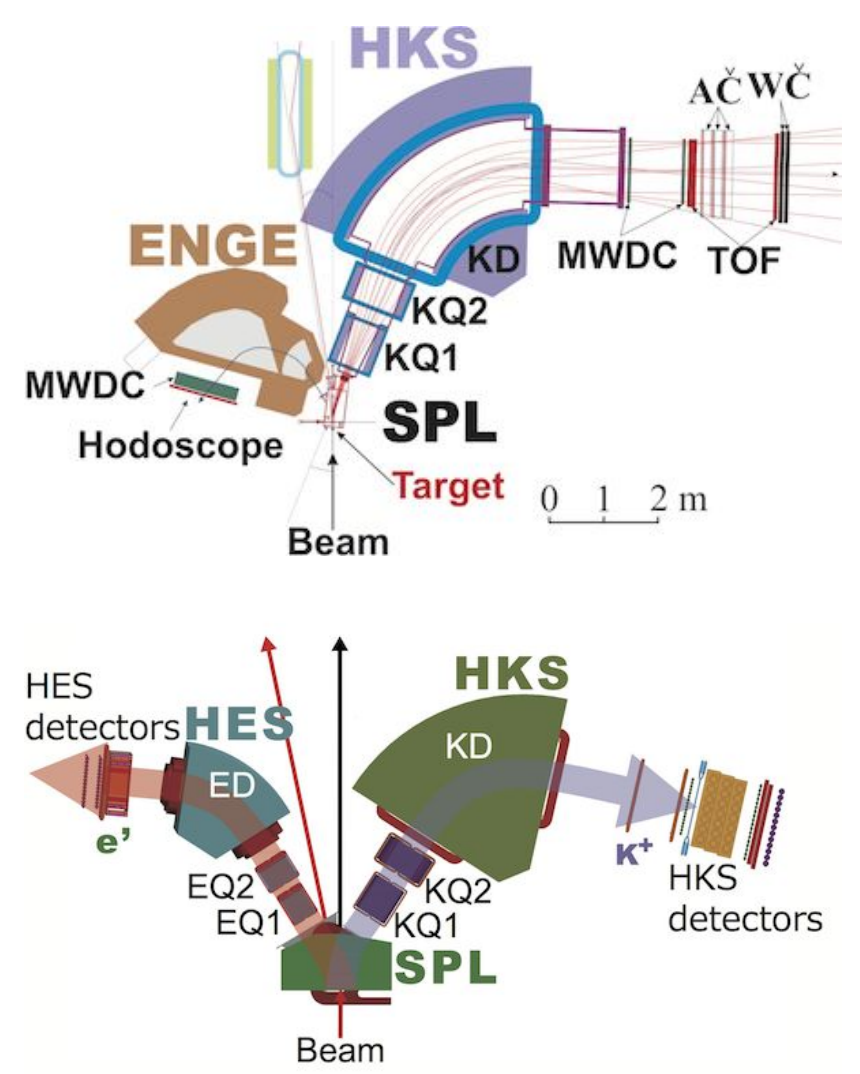

FIG. 9 Schematic illustration of the experimental setup, technique, and upgrades for the Hall C HKS hypernuclear spectroscopy experiments E01-011 (upper panel) and E05-115 (lower panel). From Tang et al., 2014.

Once the choice of the incident and scattered electron momenta is fixed, the production kaon momenta are determined by the kaon production angle. The kinematics are illustrated in Fig. 11. The recoil momentum of the $\Lambda$ is comparable to the Fermi momentum and the kaon momentum is sufficient to allow a reasonable kaon survival fraction. The detector package requires at least a 1000-to-1 kaon-to-pion particle identification. Figure 9 shows a schematic view of the experimental layout for the JLab Hall C HKS hypernuclear spectroscopy experiments E01-011 and E05-115. The splitter SPL bends electrons into the high-resolution electron spectrometer (HES) and kaons into the high-resolution kaon spectrometer (HKS), so that the reaction angles of both the electron and kaon can be observed at very forward angles. However, the SPL also bends the incident beam so that the beam must be bent back into the beam dump. This is accomplished by bending the incident beam before it enters the SPL canceling the bend angle in the SPL. In this way, the beam is bent before it is dispersed by the target, producing less scattering in magnets and apertures and thus less background. Further, to decrease the extremely high electron singles rate, the HES is rotated out of the HKS-beam dispersion plane by $7.5^{\circ}$. This tilt is equiva- 


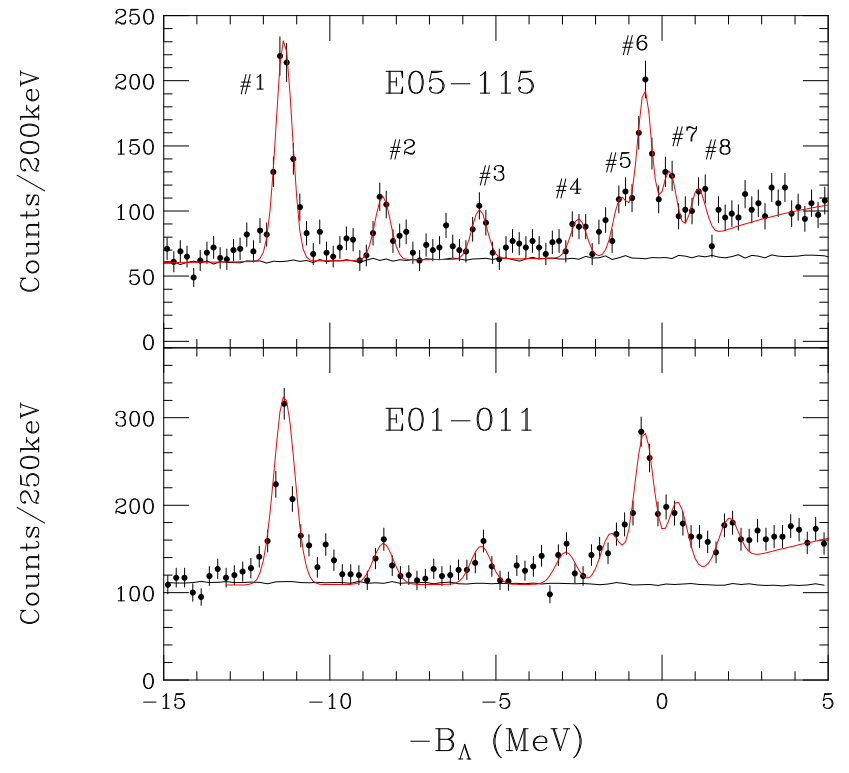

FIG. 10 Spectroscopy of ${ }_{\Lambda}^{12}$ B from the E05-115 and E01-011 experiments. The area below the black line is the accidental background. From Tang et al., 2014.

lent to a rotation plus a shift of the spectrometer so that scattered electrons $\leq 4.5^{\circ}$ hit the HES yokes and thus do not enter the spectrometer acceptance. This angle was chosen based on a figure of merit optimization between hypernuclear yield and accidental background rate. The tilt improved the true data rate by an order of magnitude while reducing accidental background. The beam and spectrometer parameters are tabulated by Tang et al. (2014). The experimental energy resolution to specific states was approximately $600 \mathrm{keV}$ FWHM.

The ${ }_{\Lambda}^{12} \mathrm{~B}$ spectrum obtained in these experiments on a ${ }^{12} \mathrm{C}$ target is shown in Fig. 10 , demonstrating the improved resolution in the more recent E05-115 experiment with respect to that in the older one E01-011 and also with respect to the Hall A experiment E94-107 (Iodice et al. 2007). In the upper panel of the figure, peaks 1, 2,3 , and 4 result from the $p_{N} \rightarrow s_{\Lambda}$ transition strength, with peak 1 standing for the ${ }_{\Lambda}^{12} \mathrm{~B}$ g.s. doublet which to a very good approximation is based on the ${ }^{11} \mathrm{~B}$ g.s. core state. The other three peaks correspond to coupling the $s_{\Lambda}$ hyperon to known excited levels in ${ }^{11} \mathrm{~B}$. Peaks 5, 6, 7 , and 8 result from the $p_{N} \rightarrow p_{\Lambda}$ transition strength which extends further up into the continuum. Similar spectra were reported for the charge-symmetric hypernucleus ${ }_{\Lambda}^{12} \mathrm{C}$ in $\left(\pi^{+}, K^{+}\right)$and $\left(K_{\text {stop }}^{-}, \pi^{-}\right)$experiments at KEK (Hotchi et al., 2001) and at DA $\Phi$ NE (Agnello et al. $2005 \mathrm{~b})$, respectively. Yet, the JLab $\left(e, e^{\prime} K^{+}\right)$experiment provides by far the most refined $A=12 \Lambda$ hypernuclear excitation spectrum.

Very recently, the spectrum of another $p$-shell hypernucleus, ${ }_{\Lambda}^{10} \mathrm{Be}$, was obtained in a JLab Hall C $\left(e, e^{\prime} K^{+}\right)$experiment (Gogami et al. 2016a). This experiment gives

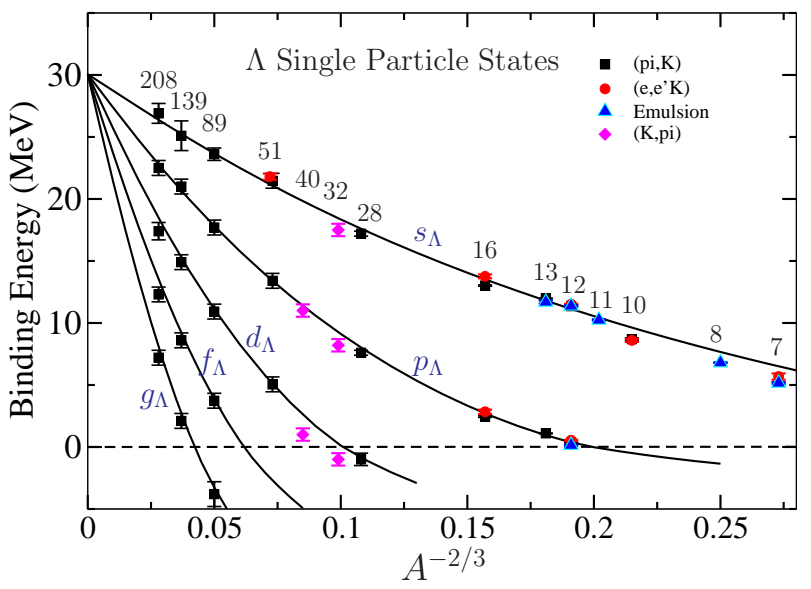

FIG. 11 Energy levels of the $\Lambda$ single-particle major shells in ${ }_{\Lambda}^{A} \mathrm{Z}$ hypernuclei as a function of $A^{-2 / 3}$. The curves are obtained from a standard Woods-Saxon potential $V_{\text {WS }}$ representing the $\Lambda$-nucleus interaction with depth $V_{0}=-30.05 \mathrm{MeV}$, radius $R=r_{0} A^{1 / 3}$, where $r_{0}=1.165 \mathrm{fm}$, and diffusivity $a=$ $0.6 \mathrm{fm}$. Updated from Millener, Dover, and Gal, 1988.

a $B_{\Lambda}$ value for a hypernucleus for which there are only a few emulsion events (see Table I). It shows four clear $s_{\Lambda}$ peaks as expected from the proton removal strength from ${ }^{10} \mathrm{~B}$ [see Sec. I.C and Fig. 3 of Millener (2012)].

The $\left(e, e^{\prime} K^{+}\right)$experiments in Hall A were performed using two existing high-relsolution (long flight path) spectrometers and used a much higher electron-beam energy of $\sim 3.7 \mathrm{GeV}$ to increase the $K^{+}$survival time. The two essential features of the setup were the placement of superconducting septum magnets before each spectrometer to be able to take data at $6^{\circ}$ and a ring-imaging Cherenkov detector to provide unambiguous $K^{+}$identification. Data were taken using targets of ${ }^{12} \mathrm{C}$ (Iodice et al. 2007), ${ }^{16} \mathrm{O}$ (Cusanno et al. , 2009), and ${ }^{9} \mathrm{Be}$ (Urciuoli et al. (2015). In particular, $B_{\Lambda}=13.76 \pm 0.16 \mathrm{MeV}$ was determined for ${ }_{\Lambda}^{16} \mathrm{~N}$ by using the $\Lambda$ and $\Sigma^{0}$ peaks from the elementary $\left(e, e^{\prime} K^{+}\right)$reaction on the hydrogen in a waterfall target for calibration.

\section{Single-particle structure}

Taking the positions of the $\Lambda$ major shells as observed in the $\left(\pi^{+}, K^{+}\right)$and other reactions, the $\Lambda$ single-particle energies show a very smooth $A$-dependence, which can be reproduced by a simple Woods-Saxon potential $V_{\mathrm{WS}}$, as shown in Fig. 11] for a data set that includes information up to ${ }_{\Lambda}^{208} \mathrm{~Pb}$ (Hasegawa et al. 1996 ). The data used in the construction of Fig. 11] is given in Table IV] Because the $B_{\Lambda}$ values in Table IV] differ in several respects from the values given in the original papers and reviews [see, e.g., Hashimoto and Tamura (2006)], some explanation is needed.

The most important overall change in the tabulated $B_{\Lambda}$ values arises from the fact that the $\operatorname{KEK}\left(\pi^{+}, K^{+}\right)$ 
TABLE IV $B_{\Lambda}$ values from a variety of sources for $\Lambda$ singleparticle states.

\begin{tabular}{|c|c|c|c|c|c|}
\hline Hypernucleus & $s_{\Lambda}$ & $p_{\Lambda}$ & $d_{\Lambda}$ & $f_{\Lambda}$ & $g_{\Lambda}$ \\
\hline & \multicolumn{5}{|c|}{$\left(\pi^{+}, K^{+}\right)$} \\
\hline${ }_{\Lambda}^{208} \mathrm{~Pb}$ & $26.9(8)$ & $22.5(6)$ & $17.4(7)$ & $12.3(6)$ & $7.2(6)$ \\
\hline${ }_{\Lambda}^{139} \mathrm{La}$ & $25.1(12)$ & $21.0(6)$ & $14.9(6)$ & $8.6(6)$ & $2.1(6)$ \\
\hline${ }_{\Lambda}^{89} \mathrm{Y}$ & $23.6(5)$ & $17.7(6)$ & $10.9(6)$ & $3.7(6)$ & $-3.8(10)$ \\
\hline${ }_{\Lambda}^{51} \mathrm{~V}$ & $21.5(6)$ & $13.4(6)$ & $5.1(6)$ & & \\
\hline${ }_{\Lambda}^{28} \mathrm{Si}$ & $17.2(2)$ & $7.6(2)$ & $-1.0(5)$ & & \\
\hline${ }_{\Lambda}^{16} \mathrm{O}$ & $13.0(2)$ & $2.5(2)$ & & & \\
\hline${ }_{\Lambda}^{13} \mathrm{C}$ & $12.0(2)$ & $1.1(2)$ & & & \\
\hline${ }_{\Lambda}^{12} \mathrm{C}$ & $11.36(20)$ & $0.36(20)$ & & & \\
\hline \multirow[t]{2}{*}{${ }_{\Lambda}^{10} \mathrm{~B}$} & $8.7(3)$ & & & & \\
\hline & \multicolumn{5}{|c|}{$\left(e, e^{\prime} K^{+}\right)$} \\
\hline${ }_{\Lambda}^{52} \mathrm{~V}$ & $21.8(3)$ & & & & \\
\hline${ }_{\Lambda}^{16} \mathrm{~N}$ & $13.76(16)$ & $2.84(18)$ & & & \\
\hline${ }_{\Lambda}^{12} \mathrm{~B}$ & $11.52(2)$ & $0.54(4)$ & & & \\
\hline${ }_{\Lambda}^{10} \mathrm{Be}$ & $8.55(13)$ & & & & \\
\hline \multirow[t]{2}{*}{${ }_{\Lambda}^{7} \mathrm{He}$} & $5.55(15)$ & & & & \\
\hline & \multicolumn{5}{|c|}{ Emulsion } \\
\hline${ }_{\Lambda}^{13} \mathrm{C}$ & $11.69(12)$ & $0.8(3)$ & & & \\
\hline${ }_{\Lambda}^{12} \mathrm{~B}$ & $11.37(6)$ & & & & \\
\hline${ }_{\Lambda}^{12} \mathrm{C}$ & & $0.14(5)$ & & & \\
\hline${ }_{\Lambda}^{8} \mathrm{Li}$ & $6.80(3)$ & & & & \\
\hline \multirow[t]{2}{*}{${ }_{\Lambda}^{7} \mathrm{Be}$} & $5.16(8)$ & & & & \\
\hline & \multicolumn{5}{|c|}{$\left(K^{-}, \pi^{-}\right)$} \\
\hline${ }_{\Lambda}^{40} \mathrm{Ca}$ & & $11.0(5)$ & $1.0(5)$ & & \\
\hline${ }_{\Lambda}^{32} \mathrm{~S}$ & $17.5(5)$ & $8.2(5)$ & $-1.0(5)$ & & \\
\hline
\end{tabular}

data were all normalized to the emulsion value of 10.76 $\mathrm{MeV}$ for ${ }_{\Lambda}^{12} \mathrm{C}$ (Hasegawa et al., 1996). This differs considerably from the emulsion value of $11.37 \mathrm{MeV}$ for ${ }_{\Lambda}^{12} \mathrm{~B}$ that is based on a substantial number of events for the characteristic $\pi^{-}+3 \alpha$ decay mode. It is generally accepted that one should not trust the emulsion $B_{\Lambda}$ values for ${ }_{\Lambda}^{12} \mathrm{C}$ and beyond because of the difficulty of identifying uniquely the decaying hypernucleus and the fact that there are very few events in each case (Davis, 1991). In fact, the best determined $B_{\Lambda}$ value for ${ }_{\Lambda}^{12} \mathrm{C}$ is $0.14(5) \mathrm{MeV}$ based on proton emission from what is interpreted as a $0^{+}$ with a dominant ${ }^{11} \mathrm{C}$ (g.s.) $\times p_{3 / 2 \Lambda}$ configuration Davis, 2008). The same analysis gives two $2^{+}$states 0.06 and $0.80 \mathrm{MeV}$ below the $0^{+}$state. These $2^{+}$states should be populated in the $\left(\pi^{+}, K^{+}\right)$spectrum with the upper one dominant. The unresolved $p_{\Lambda}$ peak from KEK E336 is $11.00(3) \mathrm{MeV}$ above the ground-state peak (Hashimoto and Tamura, 2006). Adding $0.14 \mathrm{MeV}$ and $0.23 \mathrm{MeV}$ for the difference between the $0^{+}$state and the $2^{+}$centroid gives $11.37 \mathrm{MeV}$, the same as the $B_{\Lambda}$ value for ${ }_{\Lambda}^{12} \mathrm{~B}$. Taking into account the fact that different $p_{\Lambda}$ states are populated in different reactions, one gets similar values from the $\left(e, e^{\prime} K^{+}\right)$(Iodice et al., 2007; Tang et al., 2014) and $\left(K_{\text {stop }}^{-}, \pi^{-}\right)$(Agnello et al. 2005b) reactions. Table V shows that adding $0.6 \mathrm{MeV}$ to $\left(\pi^{+}, K^{+}\right) B_{\Lambda}$ values from KEK E336 (Hashimoto and Tamura, 2006) gives better agreement with the emulsion values. However, for ${ }_{\Lambda}^{16} \mathrm{O}$
TABLE $\mathrm{V}\left(\pi^{+}, K^{+}\right)$versus emulsion $B_{\Lambda}$ values for $p$ shell hypernuclei. The first line contains values from KEK E336 (Hashimoto and Tamura, 2006), the second gives emulsion values from Table I and the last is $\left(\pi^{+}, K^{+}\right)$plus 0.6 $\mathrm{MeV}$. For comparison, Gogami et al. (2016a) have averaged the differences for ${ }_{\Lambda}^{7} \mathrm{Li},{ }_{\Lambda}^{9} \mathrm{Be},{ }_{\Lambda}^{10} \mathrm{~B}$, and ${ }_{\Lambda}^{13} \mathrm{C}$ to obtain a shift of $0.54(5) \mathrm{MeV}$.

\begin{tabular}{cccccc}
\hline \hline${ }_{\Lambda}^{7} \mathrm{Li}$ & ${ }_{\Lambda}^{9} \mathrm{Be}$ & ${ }_{\Lambda}^{10} \mathrm{~B}$ & ${ }_{\Lambda}^{12} \mathrm{C}$ & ${ }_{\Lambda}^{13} \mathrm{C}$ & ${ }_{\Lambda}^{16} \mathrm{O}$ \\
\hline $5.22(8)$ & $5.99(7)$ & $8.10(10)$ & 10.76 & $11.38(5)$ & $12.42(5)$ \\
$5.58(3)$ & $6.71(4)$ & $8.89(12)$ & $10.76(19)$ & $11.69(12)$ & \\
5.82 & 6.59 & 8.70 & 11.36 & 11.98 & 13.02 \\
\hline \hline
\end{tabular}

there is still a discrepancy with $B_{\Lambda}=13.76 \pm 0.16 \mathrm{MeV}$ for ${ }_{\Lambda}^{16} \mathrm{~N}$ (Cusanno et al., 2009).

Hasegawa et al. (1996) state in their Section II.F that they apply a shift to the $K^{+}$momentum to get the ${ }_{\Lambda}^{12} \mathrm{C}$ ground-state peak at $B_{\Lambda}=10.76 \mathrm{MeV}$. The relationship between $p_{K}$ and $B_{\Lambda}$ is linear and nearly independent of the target mass. Therefore, the energy shift applied to ${ }_{\Lambda}^{12} \mathrm{C}$ applies elsewhere. The numbers for ${ }_{\Lambda}^{28} \mathrm{Si},{ }_{\Lambda}^{139} \mathrm{La}$, and ${ }_{\Lambda}^{208} \mathrm{~Pb}$ in Table IV are from Table 13 of Hashimoto and Tamura (2006); a reanalysis of the KEK E140a data has been made and the errors include an estimate for the systematic error associated with the KEK $\left(\pi^{+}, K^{+}\right)$experiments.

Hasegawa et al. (1996) made corrections of 0.15, 0.99 , and $1.63 \mathrm{MeV}$ to the extracted $B_{\Lambda}$ values for ${ }_{\Lambda}^{89} \mathrm{Y}$, ${ }_{\Lambda}^{139} \mathrm{La}$, and ${ }_{\Lambda}^{208} \mathrm{~Pb} ; 0.15 \mathrm{MeV}$ is the centroid of the $4^{-} / 5^{-}$ $\pi p_{1 / 2}^{-1} \nu g_{9 / 2}^{-1}$ ground-state doublet of ${ }^{88} \mathrm{Y}, 0.99 \mathrm{MeV}$ is the excitation energy of the centroid of the $\nu 0 h_{11 / 2}$ pickup strength from ${ }^{139} \mathrm{La}$, and $1.63 \mathrm{MeV}$ is the excitation energy of the $\nu 0 i_{13 / 2}$ hole state in ${ }^{207} \mathrm{~Pb}$.

For ${ }_{\Lambda}^{89} \mathrm{Y}$, the left-hand peaks in Table VIII of Hotchi et al. (2001) are taken [Motoba et al. (2008) argued that the right-hand peaks are associated with the $\nu f_{5 / 2}$ hole state] whereas Hashimoto and Tamura (2006) take the centroid of the left-hand and right-hand peaks.

For ${ }_{\Lambda}^{51} \mathrm{~V}$, the left-hand peaks from Table IX of Hotchi et al. (2001) are taken and $0.9 \mathrm{MeV}$ is added because the strongest $\nu f_{7 / 2}$ pick-up strength from ${ }^{51} \mathrm{~V}$ goes to a closely spaced $7^{+} / 5^{+}$doublet at $0.9 \mathrm{MeV}$ excitation energy in ${ }^{50} \mathrm{~V}$ (levels up to about $1.3 \mathrm{MeV}$ are excited by $\nu f_{7 / 2}$ removal and the $6^{+}$ground state is also quite strong). This then gives $B_{\Lambda}=21.47 \mathrm{MeV}$ for the $s_{\Lambda}$ state, to which one should add a small amount for the increase in mass by one unit to compare with the value of 21.80 $\mathrm{MeV}$ from ${ }^{52} \mathrm{Cr}\left(e, e^{\prime} K^{+}\right){ }_{\Lambda}^{52} \mathrm{~V}$ reaction (Gogami, 2014).

For the $p_{\Lambda}$ energy in ${ }_{\Lambda}^{13} \mathrm{C}$, the centroid of the excitation energies of the $p_{3 / 2 \Lambda}(10.83 \mathrm{MeV})$ and $p_{1 / 2 \Lambda}(10.98 \mathrm{MeV})$ states from a $\gamma$-ray experiment (Kohri et al., 2002) is used.

Also included in Table IV are recent $B_{\Lambda}$ values from JLab; ${ }_{\Lambda}^{52} \mathrm{~V}$ (Gogami, 2014), ${ }_{\Lambda}^{16} \mathrm{~N}$ (Cusanno et al. 2009 ), ${ }_{\Lambda}^{12} \mathrm{~B}$ (Tang et al. 2014), ${ }_{\Lambda}^{10} \mathrm{Be}$ (Gogami et al. 2016a), and 
${ }_{\Lambda}^{7} \mathrm{He}$ Gogami et al. 2016b). The $\left(K^{-}, \pi^{-}\right)$values for ${ }_{\Lambda}^{32} \mathrm{~S}$ and ${ }_{\Lambda}^{40} \mathrm{Ca}$ are CERN data (Bertini et al. 1979 ). For ${ }_{\Lambda}^{12} \mathrm{C}$ and ${ }_{\Lambda}^{16} \mathrm{O}$, see Brückner et al. (1978) and for the summary paper (up to a ${ }^{209} \mathrm{Bi}$ target), see Bertini et al. (1981).

The data in Fig. 11 are quite well fit by a simple Woods-Saxon potential. However, when replacing $V_{\mathrm{WS}}$ by the low-density limit form $\tilde{V}_{0} \rho_{N}(r)$, with $\rho_{N}$ the nuclear density, the fit to the data requires adding a repulsive potential with a higher power of $\rho_{N}$ and, obviously, a depth $\tilde{V}_{0}$ of the attractive potential much larger than $V_{\mathrm{WS}}$ (Millener, Dover, and Gal, (1988). The resulting density-dependent $\Lambda$-nucleus potential can be traced back within a Skyrme-Hartree-Fock approach to a combination of two-body attractive $\Lambda N$ and a three-body repulsive $\Lambda N N$ interaction terms. Similar conclusions were also reached by Yamamoto, Bandō, and Žofka (1988). These early papers were based on a $\left(\pi^{+}, K^{+}\right)$experiment performed at BNL in 1987 (Pile et al., 1991). Since that time, there have been a large number of both nonrelativistic and relativistic mean-field calculations that reproduce the $\Lambda$ single-particle energies (Mareš and Jennings, 1994; Cugnon, Lejeune, and Schulze, 2000; Keil, Hoffmann, and Lenske, 2000; Vidaña et al., 2001; Finelli et al., 2009). The smooth behavior of the $B_{\Lambda}$ values is such that it should be possible to fit the updated data set in Table IV very well in almost any model with small adjustments in the parameters. In addition, the singleparticle energies have been fitted using a strongly cancelling combination of attractive $\Lambda N$ and repulsive $\Lambda N N$ interactions (Usmani and Bodmer, 1999; Lonardoni, Pederiva, and Gandolfi, 2014). These results are in some tension with the results of recent Nijmegen $Y N$ models (Yamamoto and Rijken, 2013; Nagels, Rijken, and Yamamoto, 2015b) where G-matrix folding models based just on the $Y N$ interaction fit the $\Lambda$ single-particle energies quite well.

\section{7. $\left(K^{-}, K^{+}\right)$and stopped $\Xi^{-}$reactions}

The two-body reaction $K^{-} p \rightarrow K^{+} \Xi^{-}$is the primary method used to produce double strangeness in nuclei. The forward-angle cross section of this reaction peaks for incident $K^{-}$momentum around $p_{\text {lab }}=1.8 \mathrm{GeV} / \mathrm{c}$, with a value close to $50 \mu \mathrm{b} / \mathrm{sr}$. The usefulness of the nuclear $\left(K^{-}, K^{+}\right)$reaction in producing $\Xi$ hypernuclei was discussed by Dover and Gal (1983). Missing-mass spectra on ${ }^{12} \mathrm{C}$ from experiments done at KEK (Fukuda et al., 1998) and at BNL (Khaustov et al. 2000a) are shown in Fig. 12, A full spectrum over a wide $\Xi^{-}$excitation range is shown in the upper-left diagram, and insets centered around the $\Xi^{-}$threshold are shown in the rest of the diagrams. No conclusive experimental evidence for well defined $\Xi$ hypernuclear levels could be determined because of the limited statistics and detector resolution of $\approx 10 \mathrm{MeV}$. However, by fitting to the shape and cross-section yield of the spectra in the $\Xi$-hypernuclear region, an upper bound of approximately $15 \mathrm{MeV}$ attraction was placed on the $\Xi$ hypernuclear potential strength, as shown in the figure by various calculated curves. The formation of $\Lambda \Lambda$ hypernuclei via a direct $\left(K^{-}, K^{+}\right)$reaction without intermediate $\Xi$ production is less favorable, requiring two steps, each on a different proton, e.g., $K^{-} p \rightarrow \pi^{0} \Lambda$ followed by $\pi^{0} p \rightarrow K^{+} \Lambda$ (Baltz, Dover, and Millener, 1983). The expected position of the ${ }_{\Lambda \Lambda}^{12} \mathrm{Be}$ ground state is marked by arrows for the BNL E885 experiment. Given the limited statistics, no firm evidence for the production of ${ }_{\Lambda \Lambda}^{12} \mathrm{Be}$ states was claimed.

A different class of experiments is provided by stopping $\Xi^{-}$hyperons in matter, giving rise to two $\Lambda$ 's via the twobody reaction $\Xi^{-} p \rightarrow \Lambda \Lambda$ which releases only $23 \mathrm{MeV}$. Double- $\Lambda$ hypernuclei may then be formed in stopped $\Xi^{-}$ reactions in a nuclear target, after the $\Xi^{-}$hyperons are brought to rest from a $\left(K^{-}, K^{+}\right)$reaction (Zhu et al. 1991). Calculations by Yamamoto et al., mostly using double- $\Lambda$ compound nucleus methodology, provide relative formation rates for $\Lambda \Lambda$ hypernuclei (Sano, Wakai, and Yamamoto, 1992; Yamamotoet al., 1992, 1997; Yamamoto, Sano, and Wakai, 1994).

Dedicated experiments with stopped $\Xi^{-}$hyperons were proposed in order to produce some of the lightest $\Lambda \Lambda$ hypernuclei, ${ }_{\Lambda \Lambda}^{6} \mathrm{He}$ (Zhu et al. 1991$),{ }_{\Lambda \Lambda}^{4} \mathrm{H}$ (Kumagaifuse, Koike, and Akaishi, 1995), and ${ }_{\Lambda \Lambda}^{12} \mathrm{~B}$ (Yamada and Ikeda, 1997), by searching for a peak in the outgoing neutron spectrum in the two-body reaction

$$
\Xi^{-}+{ }^{A} Z \longrightarrow{ }_{\Lambda \Lambda}^{A}(Z-1)+n .
$$

These proposals motivated the AGS experiment E885 (Khaustov et al. 2000b) which used a diamond target $\left({ }^{\text {nat }} \mathrm{C}\right)$ to stop the relatively fast $\Xi^{-}$hyperons recoiling from the quasi-free peak of the $p\left(K^{-}, K^{+}\right) \Xi^{-}$reaction in the diamond target. Non-negligible decay losses occur during the stopping time of the $\Xi^{-}$hyperon, so that a dense target was used to produce, stop, and capture the $\Xi^{-}$hyperons. An upper bound of a few percent was established for the production of the ${ }_{\Lambda \Lambda}^{12}$ Be hypernucleus. Experimental evidence for ${ }_{\Lambda}^{6} \mathrm{He}$ (Takahashi et al. 2001) and ${ }_{\Lambda \Lambda}^{4} \mathrm{H}$ (Ahn et al., 2001b) had to await different techniques, although the evidence for the latter species remains controversial (Randeniya and Hungerford 2007).

The stopped $\Xi^{-}$reaction in deuterium $\left(\Xi^{-} d\right)_{\text {atom }} \rightarrow$ $H n$ was used in AGS experiment E813 to search for the doubly strange $H$ dibaryon, yielding a negative result (Merrill et al., 2001). An earlier search by the KEK E224 collaboration, stopping $\Xi^{-}$on a scintillating-fiber active carbon target, also yielded a negative result (Ahn et al. 1996). The $\left(K^{-}, K^{+}\right)$reaction was also used, on a ${ }^{3} \mathrm{He}$ target, to establish a stringent upper limit on $H$-dibaryon production (Stotzer et al., 1997). Theoretically, based on recent lattice QCD calculations by two different groups, NPLQCD (Beane et al., 2011) and HALQCD (Inoue 


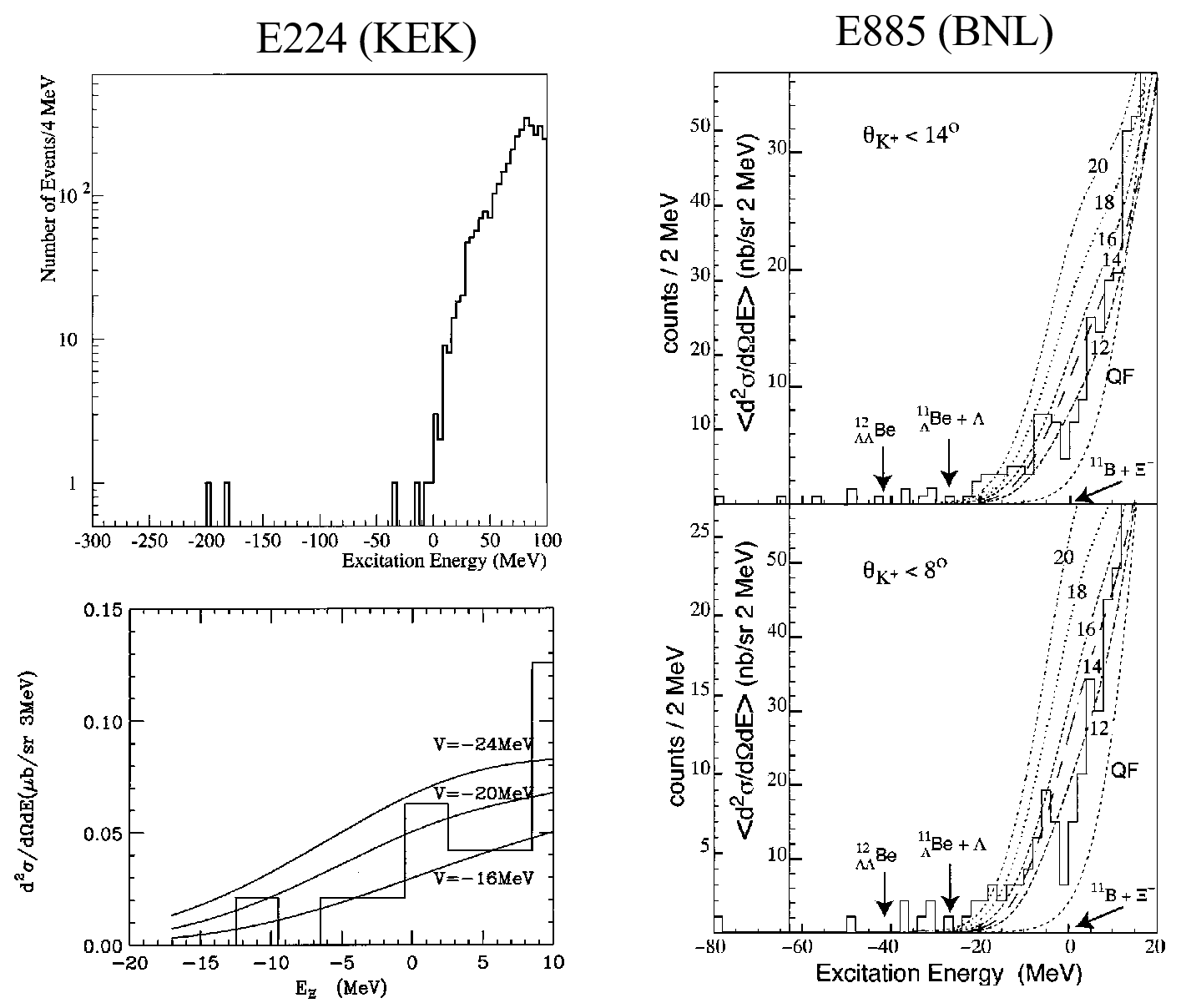

FIG. $12{ }^{12} \mathrm{C}\left(K^{-}, K^{+}\right)$missing-mass spectra measured in KEK E224 (Fukuda et al., 1998) (left) and BNL E885 (Khaustov et al., 2000a) (right). The curves correspond to assumptions made on the strength of an attractive $\Xi^{-}$-nucleus potential, folded with the experimental resolution. From Nagae, 2007.

et al. 2011), and on extrapolation made to the $\mathrm{SU}(3)$ broken hadronic world (Haidenbauer and Meißner, 2012; Inoue et al., 2012). the $\mathrm{H}$ dibaryon is unbound with respect to the $\Lambda \Lambda$ threshold, perhaps surviving in some form near the $\Xi N$ threshold.

On the positive side, a double- $\Lambda$ hypernucleus was discovered in light emulsion nuclei by the KEK stopped $\Xi^{-}$ experiment E176 (Aoki et al., 1991) and was subsequently interpreted as a ${ }_{\Lambda \Lambda}^{13} \mathrm{~B}$ hypernucleus (Dover et al., 1991; Yamamoto, Takaki, and Ikeda, 1991). This experiment produced several events, each showing a decay into a pair of known single- $\Lambda$ hypernuclei (Aoki et al., 1993, 1995). Two more events were reported by the KEK E373 collaboration (Ichikawa et al., 2001, Nakazawa et al., 2015), with the latter event claimed to imply a lightly bound $\Xi^{-}{ }^{14} \mathrm{~N}$ nuclear state. Using these events, one should be able to deduce the properties of the initial $\Xi^{-}$atomic states. However, the $100 \mathrm{keV}$ resolution common in emulsion work is 3 orders of magnitude larger than typical values anticipated for the strong-interaction shifts and widths of $\Xi^{-}$atomic levels. This provides a major justification for pursuing a program for the measurement of
$\Xi^{-}$X rays (Batty, Friedman, and Gal, 1999), in parallel with strong-interaction reactions involving $\Xi$ hyperons.

\section{Hypernuclear lifetime measurements}

If the velocity of a hypernucleus recoiling from a production reaction is known, its lifetime can be measured by the distance it travels before decaying. This recoildistance technique was used to observe and measure the lifetime of many short lived particles. In particular the lifetime of a free, unbound $\Lambda,(263 \pm 2)$ ps (Olive et al., 2014), was determined by observing its mesonic decay in a beam of neutrally charged hyperons (Poulard, Givernaud, and Borg, 1973; Clayton et al., 1975; Zech et al., 1977).

Lifetimes of ${ }_{\Lambda}^{3} \mathrm{H},{ }_{\Lambda}^{4} \mathrm{H}$ and ${ }_{\Lambda}^{5} \mathrm{He}$ measured in emulsion were published as early as in 1964 (Prem and Steinberg, 1964), but since hypernuclei are generally produced in emulsion with low kinetic energies, only very few decayed in flight, incurring relatively large experimental uncertainties on the deduced lifetimes. The more precise 
TABLE VI $\Lambda$ hypernuclear lifetimes (in ps) measured at KEK, using $\left(\pi^{+}, K^{+}\right)$production reactions.

\begin{tabular}{l|c|c|c|c}
\hline \hline$\Lambda$ & ${ }_{\Lambda}^{5} \mathrm{He}$ & ${ }_{\Lambda}^{12} \mathrm{C}$ & ${ }_{\Lambda}^{28} \mathrm{Si}$ & ${ }_{\Lambda} \mathrm{Fe}$ \\
\hline $263 \pm 2 \mathrm{a}$ & $278 \pm 11^{\mathrm{b}}$ & $212 \pm 7^{\mathrm{b}}$ & $206 \pm 11^{\mathrm{c}}$ & $215 \pm 14^{\mathrm{c}}$ \\
\hline \hline
\end{tabular}

a Olive et al. (2014).

b Kameoka et al. (2005).

c Bhang et al. (1998) and Park et al. (2000).

${ }_{\Lambda}^{3} \mathrm{H}$ lifetime deduced in a subsequent emulsion measurement, $\tau\left({ }_{\Lambda}^{3} \mathrm{H}\right)=128_{-26}^{+35}$ ps (Bohm et al. 1970a $)$, is considerably shorter than the one deduced from a helium bubblechamber measurement, $\tau\left({ }_{\Lambda}^{3} \mathrm{H}\right)=246_{-41}^{+62}$ ps (Keyes et al. 1973). The latter is equal to the free $\Lambda$ lifetime within the experimental uncertainties. This was explained by Bohm and Wysotski (1970) as the possible Coulomb dissociation of the very weakly bound ${ }_{\Lambda}^{3} \mathrm{H}$ when traversing the high- $Z$ emulsion. Finally, the ${ }_{\Lambda}^{5}$ He lifetime deduced in that emulsion study (Bohm et al., 1970b) agrees perfectly within its larger uncertainties with the lifetime deduced 35 years later in a KEK experiment in which ${ }_{\Lambda}^{5} \mathrm{He}$ was produced in a $\left(\pi^{+}, K^{+}\right)$reaction (Kameoka et al., 2005). This and other lifetimes measured similarly at KEK are listed in Table VI with ${ }_{\Lambda} \mathrm{Fe}$ the heaviest $\Lambda$ hypernucleus for which this information is available. It is clear from the table that beginning with ${ }_{\Lambda}^{12} \mathrm{C}$ the $\Lambda$ hypernuclear lifetimes saturate at a value about $80 \%$ of the free $\Lambda$ lifetime.

The first accelerator experiment to apply the recoildistance method in a hypernuclear experiment used the LBL Bevatron to produce a hypernuclear beam by bombarding a polyethylene target with a $2.1 \mathrm{GeV} /$ nucleon ${ }^{16} \mathrm{O}$ beam (Nield et al. 1976). Spark chamber detectors with photographic readout were positioned behind the target and scanned for tracks with a decay vertex. The readout trigger required that an interaction occurred in the target and a potential decay was observed within a given time delay. These events were analyzed by a fit to the form $N(x)=A \exp (-x / \lambda)+B$ by varying $A, B$ and $\lambda$, where $B$ is a constant background, $\lambda$ the mean lifetime of the hypernucleus, and $x$ the mesured distance between the vertex and the target. Although the actual system which decayed was not directly identified, the most likely hypernuclear production reactions were assumed to be

$$
\begin{aligned}
& { }^{16} \mathrm{O}+p \rightarrow{ }_{\Lambda}^{16} \mathrm{O}+n+K^{+}, \\
& { }^{16} \mathrm{O}+n \rightarrow{ }_{\Lambda}^{16} \mathrm{~N}+n+K^{+} .
\end{aligned}
$$

The measured mean life was found to be $86_{-26}^{+33} \mathrm{ps,} \mathrm{which}$ is two to three times shorter than lifetimes measured in this hypernuclear mass range in more recent, better controlled $\left(\pi^{+}, K^{+}\right)$experiments at KEK (Bhang et al. 1998, Park et al., 2000), as demonstrated in Table|VI.

More recently, the HypHI Collaboration at GSI reported lifetimes of ${ }_{\Lambda}^{3} \mathrm{H}$ and ${ }_{\Lambda}^{4} \mathrm{H}$ produced by bombarding

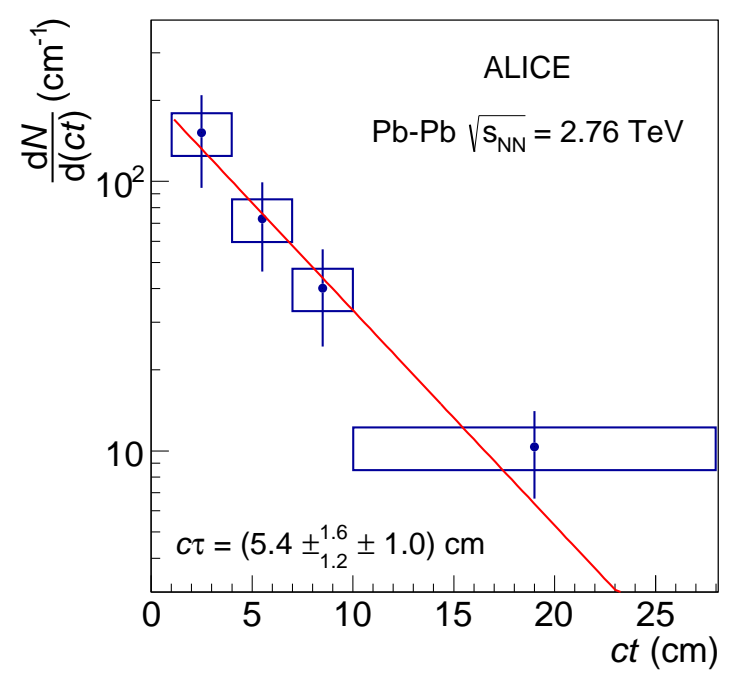

FIG. 13 Measured $\mathrm{d} N / \mathrm{d}(c t)$ distribution and exponential fit used by the ALICE Collaboration to determine the lifetime of ${ }_{\Lambda}^{3} \mathrm{H}$ produced in $\mathrm{Pb}-\mathrm{Pb}$ central collisions at $\sqrt{s_{N N}}=2.76 \mathrm{TeV}$ at the CERN-LHC. The bars and boxes are statistical and systematic uncertainties, respectively. Adapted from Adam et al., 2016a.

a carbon target with a $2 \mathrm{GeV} /$ nucleon ${ }^{6} \mathrm{Li}$ beam (Rappold et al. 2013a). The lifetime of ${ }_{\Lambda}^{3} \mathrm{H}$ has also been measured in heavy-ion central collisions, by the STAR Collaboration at the BNL-RHIC collider (Abelev et al. 2010) and by the ALICE Collaboration at CERN-LHC (Adam et al., 2016a). These measurements use the time dilation of a Lorentz boost to the recoiling hypernucleus produced in the collision, as shown in Fig. 13 from the ALICE determination of $\tau\left({ }_{\Lambda}^{3} \mathrm{H}\right)$. The values deduced from these measurements for the ${ }_{\Lambda}^{3} \mathrm{H}$ lifetime are about $25 \%$ shorter than the free $\Lambda$ lifetime, see the latest compilation by Rappold et al. (2014). This poses a serious theoretical challenge as discussed later in Sec. VIII.D.3.

Several programs have attempted to obtain the lifetime of heavy hypernuclei using the recoil-distance method for delayed fission after stopping antiprotons on $\mathrm{Bi}$ and $\mathrm{U}$ targets (Bocquet et al., 1987; Armstrong et al., 1993) or by electroproduction on a Bi target (Noga et al., 1986). These use back-to-back fission fragments from the presumed decay of a recoiling hypernucleus to obtain the position of the decay relative to the target. As previously, the recoil velocity and decay position provide the hypernuclear lifetime.

As an example, this technique was used by the COSY13 Collaboration to obtain the lifetime of hypernuclei averaged over hypernuclear masses from $A=160-190,170$ 200 , and 200-230. The data were obtained from the fission of nuclear systems recoiling from an approximately $1.9 \mathrm{GeV}$ proton beam incident on $\mathrm{Au}, \mathrm{Bi}$, and $\mathrm{U}$ tar- 
gets, respectively (Pysz et al., 1999; Cassing et al., 2003). Obviously the specific recoiling system was unknown, so the masses and momenta of the recoils were obtained from coupled-channel transport and statistical evaporation models. In both the COSY-13 and $\bar{p}$ experiments, fragments and particles emitted directly from the target were blocked from entering the amplitude-sensitive fission detectors - the recoil shadow method. The result of the COSY-13 experiment was a lifetime of $(145 \pm 11)$ ps. This is significantly shorter than the lifetime expected by extrapolating the measured lifetimes listed in Table VI which indicate that saturation of hypernuclear lifetimes is achieved already for $A \geq 12$. Cassing et al. (2003) argued that the result shows significant violation of the $\Delta I=1 / 2$ rule. However, Bauer and Garabarino (2010) pointed out that no known mechanism could account for this significant decrease in the lifetime compared to $(215 \pm 14)$ ps measured for ${ }_{\Lambda} \mathrm{Fe}$ (Bhang et al., 1998; Park et al. 2000; Sato et al. 2005). Therefore, additional, more constrained measurements are needed to resolve this controversy.

\section{G. Free-space and in-medium $Y N$ interactions}

\section{The free-space $Y N$ interaction}

One of the motivations for the study of hypernuclei was the expectation that information on the low-energy $\Lambda N$ interaction could be extracted from the spins and binding energies of the $s$-shell hypernuclear systems. Direct scattering and reaction measurements involving $\Lambda$ 's are extremely difficult, since the $\Lambda$ is electrically neutral and its lifetime is short, $\approx 263$ ps. Thus, production and scattering must be done in the same target, and the detector must have sufficient granularity and particle identification to analyze scattering events in the presence of a number of possible backgrounds. The data that do exist comes mostly from hydrogen bubble chambers, and was acquired with a stopping $K^{-}$beam. Hence, the data analysis must extract the kinematics and rates from tracks in the bubble-chamber target as the $\Lambda$ recoils from the $p\left(K_{\text {stop }}^{-}, \pi^{0}\right) \Lambda$ reaction and then scatters from another hydrogen nucleus (Alexander et al., 1968, Sechi-Zorn et al. 1968).

There are also a few data points for $\Sigma p$ scattering and reactions (Eisele et al. 1971$)$ taken using hydrogen bubble chambers. However, a more recent technique used a scintillating-fiber target (Ahn et al., 1999), applying the $\left(\pi^{+}, K^{+}\right)$reaction to produce and scatter $\Sigma^{+}$'s in the scintillating fiber. This technique tracks the charged $\Sigma^{+}$'s to, and after, their interactions with protons in the fibers by observing electronically stored, stereo images of reaction events. The readout is triggered by a $(\pi, K)$ spectrometer system that identifies the possible production of $\Sigma^{+}$recoils that could have re-scattered (Ahn et al.
1999). One might envision using a similar apparatus to obtain $\Lambda N$ scattering data, but inferring the energy and scattering angle of a neutral $\Lambda$ is not feasible.

Excluding the latest $\Sigma p$ data, there are some $37 Y N$ (hyperon-nucleon) data points. Obviously this is insufficient to extract even the scattering lengths, so these data are analyzed using models of $\mathrm{SU}(3)_{\mathrm{f}}$ symmetry of the baryon-baryon interaction that make connections with the richer $N N$ data. However, $\mathrm{SU}(3)_{\mathrm{f}}$ is badly broken due to the difference in mass between the $s$ and $(u, d)$ quarks, so that realistic models must include $\mathrm{SU}(3)_{\mathrm{f}}$ breaking terms. Several $Y N$ potential models have been developed along these lines for use in hypernuclear physics. The most used ones are as follows:

- The Nijmegen models, including the hard-core models D (Nagels, Rijken, and de Swart, 1977) and F (Nagels, Rijken, and de Swart, 1979), the soft-core models NSC89 (Maessen, Rijken, and de Swart, 1989) and NSC97 (Rijken, Stoks, and Yamamoto, 1999), and the extended soft core models ESC04 (Rijken and Yamamoto, 2006a) and ESC08 (Nagels, Rijken, and Yamamoto, 2015b) that, in addition to one boson exchange (OBE), also consider pseudoscalar (PS) two-meson exchanges and other short-range contributions. These models in particular allow extension to hyperon-hyperon $(Y Y)$ potentials where there is almost no scattering data (Nagels, Rijken, and Yamamoto, 2015a) implying unfortunately an increased model dependence. For applications to $S=-2$ hypernuclei, see Yamamoto and Rijken (2008).

- The Bonn-Jülich multi-meson-exchange models (Holzenkamp, Holinde, and Speth, 1989; Reuber, Holinde, and Speth, 1994; Haidenbauer and Meißner, 2005) that are based on the SU(6) symmetry of the quark model. The short-range behavior of the $Y N$ interaction in these and in the Nijmegen models follows largely from the way scalar-meson interactions are introduced, and is therefore necessarily model dependent.

- Effective Field Theory (EFT) chiral models, of leading order (LO) (Polinder, Haidenbauer and Meißner, 2006) and next to leading order (NLO) (Haidenbauer et al., 2013), that use regularized PS Goldstone-boson exchange $Y N$ potentials, adding zero-range contact terms to parametrize the shortrange behavior of the $Y N$ coupled-channel interactions. For a recent review see Haidenbauer (2013).

In addition, a quark-model baryon-baryon potential obeying SU(6) symmetry was developed by Fujiwara, Suzuki, and Nakamoto (2007) and used for constructing hyperon-nucleus potentials (Kohno and Fujiwara, 2009).

Table VII compares the $\Lambda N$ singlet and triplet scattering lengths and effective ranges for several models, show- 
TABLE VII $\Lambda N$ scattering lengths and effective ranges (in fm) for several $Y N$ interaction models. For the EFT models, these refer to $\Lambda p$ and to cutoff parameter of $600 \mathrm{MeV}$.

\begin{tabular}{llrrr}
\hline \hline Model & \multicolumn{1}{c}{ Reference } & $a^{s}$ & $r_{0}^{s}$ & $a^{t}$ \\
\hline NSC89 & Maessen, Rijken, and de Swart (1989) & -2.79 & 2.89 & -1.36 \\
NSC97e & Rijken, Stoks, and Yamamoto (1999) & -2.17 & 3.22 & -1.84 \\
NSC97f & Rijken, Stoks, and Yamamoto (1999) & -2.60 & 3.05 & -1.71 \\
ESC08c & Nagels, Rijken, and Yamamoto (2015b) & -2.54 & 3.15 & -1.72 \\
Jülich '04 & Haidenbauer and Meißner (2005) & -2.56 & 2.75 & -1.66 \\
EFT (LO) & Polinder, Haidenbauer, and Meißner (2006) & -1.91 & 1.40 & -1.23 \\
EFT (NLO) & Haidenbauer et al. (2013) & -2.91 & 2.78 & -1.54 \\
\hline \hline
\end{tabular}

ing that the $Y N$ low-energy data cannot determine precisely these low-energy parameters. Judging by the $\Lambda N$ scattering lengths, the $\Lambda N$ interaction is attractive but is weaker roughly by a factor of 2 than the $N N$ interaction. This is consistent with the absence of $\Lambda N$ bound states and with the onset of $\Lambda$-hypernuclear binding realized by the weakly-bound hypertriton ${ }_{\Lambda}^{3} \mathrm{H}\left(B_{\Lambda}=0.13 \pm 0.05 \mathrm{MeV}\right.$, see Table If). The spin dependence of the $\Lambda N$ interaction is opposite to that of the $N N$ interaction, with the spinsinglet s-wave $\Lambda N$ interaction being stronger than the spin-triplet interaction, consistent with the known spinparity, $J^{\pi}=\frac{1}{2}^{+}$, of ${ }_{\Lambda}^{3} \mathrm{H}$.

\section{Extraction of $\Lambda N$ interaction in final-state interactions}

Extraction of the $N N$ scattering lengths and effective ranges from scattering of nucleons in a continuum final state has been throughly explored. The technique has been used to compare neutron-neutron to proton-neutron and proton-proton scattering in order to obtain charge symmetry breaking information (Gross, Hungerford, and Malanify, 1971). The experiments analyze the spectrum of a three-body breakup reaction in the region of phase space where two final-state nucleons have low relative energy. They require excellent energy resolution, but only relative cross sections.

Extension of this technique to obtain the $Y N$ scattering lengths and effective ranges has also been proposed (Karplus and Rodberg, 1959; Gibbs et al., 2000). Experimentally, one must have an energy resolution $\ll 1 \mathrm{MeV}$ near the turning point in phase space where the reduced energy of the hyperon and nucleon vanishes. This is not presently possible in mesonic production reactions, and while sub-MeV resolution of hypernuclear spectra may be obtained in electromagnetic production, quasi-free $\Sigma$ production is high, and unfavorable kinematic conditions due to the light mass of the recoiling $\Lambda N$ system significantly reduce the resolution. The sensitivity of the spectrum shape to the effective range (Dohrmann et al. 2007) is thus degraded.

\section{Comparison of the $\Lambda N$ and $\Sigma N$ interactions}

The $\Lambda$ has isospin 0 , so the $\Lambda N$ interaction occurs only in the isospin state $I_{Y N}=1 / 2$ only. On the other hand, the $\Sigma$ hyperon has isospin 1, allowing $\Sigma N$ interaction in both isospin states $I_{Y N}=1 / 2$ and $3 / 2$. Although $Y N$ input data are limited, the Nijmegen, and the EFT potentials in particular, favor significant $\Sigma N$ spin and isospin dependence, yielding strong attraction in the ${ }^{1} S_{0}$, $T=3 / 2$ and ${ }^{3} S_{1}, T=1 / 2$ channels and repulsion in the ${ }^{3} S_{1}, T=3 / 2$ and ${ }^{1} S_{0}, T=1 / 2$ channels. This is rather different from the relatively weak spin dependence of the attractive $\Lambda N$ interaction in these models. Perhaps the most significant difference between the $\Lambda N$ and $\Sigma N$ interactions is the strong conversion $\Sigma N \rightarrow \Lambda N$ with energy release of some $80 \mathrm{MeV}$. This dominates the behavior of a $\Sigma$ in the nuclear medium (Dover, Millener, and Gal, 1989), implying also appreciable $\Lambda N \leftrightarrow \Sigma N$ mixing, particularly in the $s$-shell hypernuclei, as discussed in Sec. II.A.1.

\section{The effective $Y N$ interaction}

The hyperon-nucleon interaction involves the coupled $\Lambda \mathrm{N}$ and $\Sigma \mathrm{N}$ channels, as illustrated in Fig. 14. The diagrams in the figure make the point that the direct $\Lambda N-\Lambda N$ interaction does not contain a one-pionexchange contribution because of isospin conservation (except for electromagnetic violations via $\Lambda-\Sigma^{0}$ mixing) while the coupling between the $\Lambda N$ and $\Sigma N$ channels does. For this reason alone, the $\Lambda N$ interaction is considerably weaker than the $N N$ interaction, and there is reason to believe that the three-body $\Lambda N N$ interaction in a hypernucleus could be relatively important.

The free-space interactions are obtained as extensions of meson-exchange models for the $N N$ interaction by invoking, e.g., a broken flavor, $\mathrm{SU}(3)_{\mathrm{f}}$, symmetry. The most widely used model is the Nijmegen soft-core, oneboson-exchange potential model known as NSC97 (Rijken, Stoks, and Yamamoto, 1999). The six versions of this model, labeled NSC97a-f, cover a wide range of possibilities for the strength of the central spin-spin interaction ranging from a triplet interaction that is stronger 

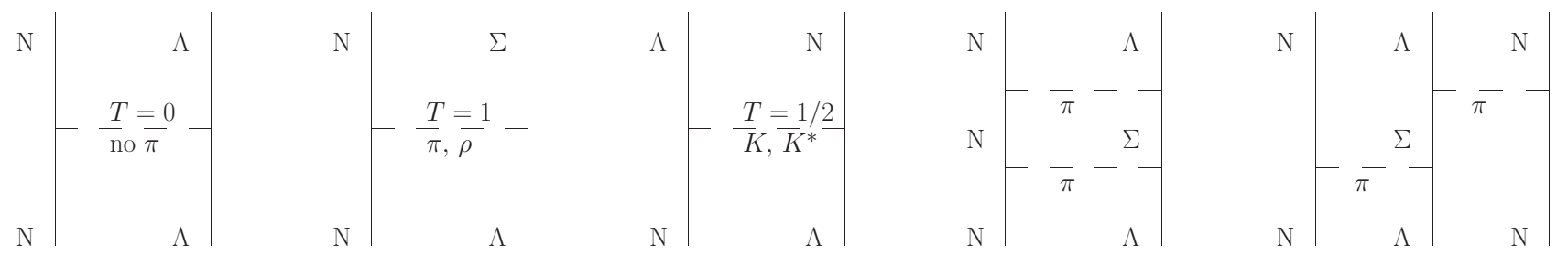

FIG. 14 Diagrams showing schematically the important features of the coupled $\Lambda N-\Sigma N$ strangeness -1 interaction for isospin $1 / 2$. All diagrams are $Y N$ Born diagrams. The first diagram represents generically meson exchanges such as $\eta$ and $\omega$, and the fourth diagram iterates the one-pion exchange of the second diagram and is included in the standard coupled-channels approach to the $Y N$ interaction. The last diagram shows a two-pion-exchange three-body interaction.

than the singlet interaction to the opposite situation. More recently, extended soft-core versions, ESC04 (Rijken and Yamamoto, 2006a) and ESC08 (Nagels, Rijken, and Yamamoto, 2015b), have become available. Effective interactions for use in a nuclear medium are then derived through a G-matrix procedure (Rijken, Stoks, and Yamamoto, 1999; Rijken and Yamamoto, 2006a; Yamamoto, Motoba, and Rijken, 2010).

The $\Lambda N$ effective interaction can be written (neglecting a quadratic spin-orbit component) in the form

$$
\begin{aligned}
V_{\Lambda N}(r)= & V_{0}(r)+V_{\sigma}(r) \boldsymbol{s}_{N} \cdot \boldsymbol{s}_{\Lambda}+V_{\Lambda}(r) \boldsymbol{l}_{N \Lambda} \cdot \boldsymbol{s}_{\Lambda} \\
& +V_{N}(r) \boldsymbol{l}_{N \Lambda} \cdot \boldsymbol{s}_{N}+V_{T}(r) S_{12}
\end{aligned}
$$

where $V_{0}$ is the spin-averaged central interaction, $V_{\sigma}$ is the difference between the triplet and singlet central interactions, $V_{\Lambda}$ and $V_{N}$ are the sum and difference of the strengths of the symmetric spin-orbit (SLS) interaction $\boldsymbol{l}_{N \Lambda} \cdot\left(\boldsymbol{s}_{\Lambda}+\boldsymbol{s}_{N}\right)$ and antisymmetric spin-orbit (ALS) interaction $\boldsymbol{l}_{N \Lambda} \cdot\left(\boldsymbol{s}_{\Lambda}-\boldsymbol{s}_{N}\right)$, and $V_{T}$ is the tensor interaction with

$$
S_{12}=3\left(\boldsymbol{\sigma}_{N} \cdot \hat{\boldsymbol{r}}\right)\left(\boldsymbol{\sigma}_{\Lambda} \cdot \hat{\boldsymbol{r}}\right)-\boldsymbol{\sigma}_{N} \cdot \boldsymbol{\sigma}_{\Lambda}
$$

For the $\Lambda$ in an $s$ orbit, $\boldsymbol{l}_{N \Lambda}$ is proportional to $\boldsymbol{l}_{N}$ (Gal, Soper, and Dalitz, 1971). The effective $\Lambda N-\Sigma N$ and $\Sigma N-\Sigma N$ interactions can be written in the same way.

Effective interactions in common use are the hyperonnucleon Gaussian (YNG) interactions (Yamamoto et al., 1994; Yamamoto, Motoba, and Rijken, 2010) in which each term is represented by an expansion in terms of a limited number of Gaussians with different ranges,

$$
V(r)=\sum_{i} v_{i} e^{-r^{2} / \beta_{i}^{2}}
$$

for the central and spin-orbit components, and

$$
V_{T}(r)=\sum_{i} v_{i} r^{2} e^{-r^{2} / \beta_{i}^{2}}
$$

for the tensor component. When based on nuclear-matter calculations, the YNG matrix elements are made density dependent by parametrizing the coefficients $v_{i}$ through the Fermi momentum $k_{F}$.
Effective interactions for finite nuclei, specifically for $p$ shell hypernuclei, have been generated using a BruecknerHartree procedure (Halderson, 2008). These use Yukawa forms in place of the Gaussians above, are densityindependent, and are available for most of the Nijmegen interactions (D. Halderson, private communication). The above Gaussian or Yukawa interactions provide a starting point for the interactions that give rise to the parameter sets in Eqs. (23)- 25 describing the energy spectra of $p$-shell hypernuclei. This process is illustrated in Millener (2010), which also contains some remarks about the possible role of the double one-pion exchange $\Lambda N N$ interaction (see Fig. 14 introduced long ago for $p$-shell hypernuclei by Gal, Soper, and Dalitz (1971). Phenomenological, but physically motivated, $\Lambda N N$ interactions have been used for the $s$-shell hypernuclei and the $\Lambda$ well depth (Bodmer, Usmani, and Carlson, 1984a; Bodmer and Usmani, 1988). These studies were later extended to a full study of $\Lambda$ single-particle energies (Usmani and Bodmer, 1999), most recently using auxilliary-field diffusion Monte Carlo techniques (Lonardoni, Pederiva, and Gandolfi, 2014). While microscopically derived $Y N N$ interactions have not been available for use in few-body calculations (Nogga, 2013), such interactions have been recently derived from $\mathrm{SU}(3)$ chiral effective field theory (Petschauer et al. 2016) but not yet applied.

\section{II. $\Lambda$ HYPERNUCLEI}

\section{A. Structure calculations}

\section{1. $s$-shell hypernuclei}

The $s$-shell hypernuclei illustrate many of the features of the $\Lambda N$ interaction (Dalitz, Herndon, and Tang, 1972; Nemura, Akaishi, and Suzuki, 2002; Nogga, Kamada, and Glöckle, 2002). The binding energy of the lightest hypernucleus, the hypertriton ${ }_{\Lambda}^{3} \mathrm{H}$, was obtained from emulsion (Bohm et al., 1968; Jurič et al., 1973; Davis and Pniewski, 1986). Its spin and parity, $J^{\pi}=1 / 2^{+}$ (Dalitz, 1969; Keyes et al., 1970), was found by analysis of its $\pi^{-}$weak-decay width (Dalitz, 1958; Dalitz and Liu, 1959). As a consequence, one can deduce that the 
spin-singlet, as opposed to the spin-triplet, $\Lambda N$ interaction must be stronger. In addition, as the binding energy (Davis and Pniewski, 1986) is only $0.13 \pm 0.05 \mathrm{MeV}$, there is no bound, excited $T=0$ hypertriton state. A bound $T=1 \Lambda n n$ was speculated recently by the HypHI Collaboration at GSI (Rappold et al. 2013b). However, $A=3$ few-body calculations constrained by the $T=0$ hypertriton (Miyagawa et al., 1995; Belyaev, Rakityansky, and Sandhas, 2008; Gal and Garcilazo, 2014; Garcilazo and Valcarce, 2014; Hiyama et al., 2014), and in some also by the $A=4$ hypernuclei Gal and Garcilazo, 2014. Hiyama et al. 2014), rule out a bound $\Lambda$ nn. The spin-parity and binding energy of the hypertriton provide important constraints on the spin components of the central $\Lambda N$ potential because of the lack of direct data from low-energy $\Lambda N$ scattering (Downs and Dalitz, 1959; Dalitz, 1969).

The binding energies of the $A=4$ hypernuclei, ${ }_{\Lambda}^{4} \mathrm{H}$ and ${ }_{\Lambda}^{4} \mathrm{He}$, have been extracted from emulsion data (Bohm et al. 1968, Jurič et al. 1973 ). Spin assignments of these hypernuclei were obtained from analysis of their pionic weak decays (Dalitz, 1958, Downs and Dalitz, 1959). This isodoublet of hypernuclei forms the lightest system of isobaric mirror hypernuclei and provides information on charge-symmetry breaking (CSB) in the $\Lambda N$ interaction. The excited states of these hypernuclei were observed at $\sim 1.1 \mathrm{MeV}$ by stopping $K^{-}$mesons in $\mathrm{Li}$ isotopes and looking for hypernuclear $\gamma$ transitions to the $A=4$ ground states in coincidence with either their $\pi^{-}$or $\pi^{0}$ weak decays (Bamberger et al. 1973, Bedjidian et al. 1979). A very recent J-PARC experiment, E13 (Tamura et al. 2013), using the in-flight $\left(K^{-}, \pi^{-}\right)$reaction directly on ${ }^{4} \mathrm{He}$, identifies the $M 1 \gamma$ ray transition in ${ }_{\Lambda}^{4} \mathrm{He}$ at $E_{\gamma}=1.41 \mathrm{MeV}$ (Yamamoto et al. 2015), thereby implying that the $1^{+}$excitation energy in ${ }_{\Lambda}^{4} \mathrm{He}$ is $1.41 \mathrm{MeV}$, which differs substantially from the $1.15 \mathrm{MeV}$ (Bedjidian et al., 1979) traditionally accepted, see Fig. 15. The resulting CSB in the $1^{+}$excited states in the $A=4$ hypernuclei is then considerably smaller than in the $0^{+}$ground states. Also recently, the binding energy of ${ }_{\Lambda}^{4} \mathrm{H}$ was determined to be $B_{\Lambda}=2.12 \pm 0.01$ (stat) \pm 0.09 (syst) MeV at Mainz by measuring the momentum of the monochromatic pion from the two-body decay ${ }_{\Lambda}^{4} \mathrm{H} \rightarrow{ }^{4} \mathrm{He}+\pi^{-}$(Esser et al. 2015$)$. This is consistent, within the systematic error, with the emulsion value shown in Fig. 15.

The heaviest of the $s$-shell hypernuclei is ${ }_{\Lambda}^{5} \mathrm{He}$. It has a spin-parity of $J^{\pi}=1 / 2^{+}$(Dalitz, 1958) and has no bound excited state. Its binding energy (Bohm et al., 1968$)$ of $3.12 \pm 0.02 \mathrm{MeV}$ is anomalously low as has been noted for many years (Dalitz, Herndon, and Tang, 1972; Dalitz, 1973; Hungerford and Biedenharn, 1984). A straightforward calculation with just a $\Lambda N$ interaction using the measured binding energy values of the $A=3$, and 4 systems (including the excited states) as calibrations, overbinds ${ }_{\Lambda}^{5} \mathrm{He}$ by $1-2 \mathrm{MeV}$. Conversely, fitting to ${ }_{\Lambda}^{3} \mathrm{H}$ and

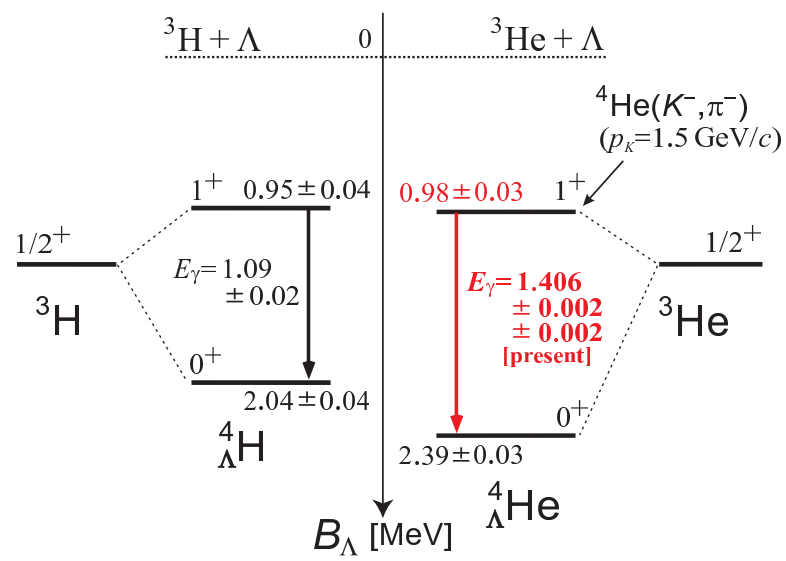

FIG. 15 Level diagrams for the $A=4 s$-shell hypernuclei showing the ground-state binding energies from emulsion data and incorporating information on the ${ }_{\Lambda}^{4} \mathrm{He}$ excited state from a $\gamma$-ray experiment with the Hyperball-J at J-PARC (energies in $\mathrm{MeV}$ ). From Yamamoto et al., 2015.

${ }_{\Lambda}^{5}$ He leaves the $A=4$ hypernuclei underbound. This problem has been attributed to a $\Lambda N$ tensor force, a threebody force, $\Lambda-\Sigma$ coupling, and partial quark deconfinement.

The importance of $\Lambda-\Sigma$ coupling in this regard has been simply demonstrated by writing two-component wave functions for either the $0^{+}$or the $1^{+}$states of ${ }_{\Lambda}^{4} \mathrm{He}$ (or ${ }_{\Lambda}^{4} \mathrm{H}$ ) with isospin $T=1 / 2$ (Akaishi et al. 2000)

$$
\left|{ }_{\Lambda}^{4} \mathrm{He}\right\rangle=\alpha s^{3} s_{\Lambda}+\beta s^{3} s_{\Sigma} .
$$

The $\Sigma$ component is $2 / 3 \Sigma^{+}$and $1 / 3 \Sigma^{0}$ for ${ }_{\Lambda}^{4} \mathrm{He}\left(2 / 3 \Sigma^{-}\right.$ and $1 / 3 \Sigma^{0}$ for $\left.{ }_{\Lambda}^{4} \mathrm{H}\right)$. The off-diagonal matrix elements $v(J)(J=0,1)$ between the basis states can be derived from the $\Lambda \mathrm{N}-\Sigma \mathrm{N}$ G matrix for $0 s$ orbits, giving (Akaishi et al. 2000, Millener, 2007)

$$
\begin{aligned}
& v(0)=\frac{3}{2}{ }^{3} g-\frac{1}{2} 1 g=\overline{\mathrm{V}}^{\prime}+\frac{3}{4} \Delta^{\prime}, \\
& v(1)=\frac{1}{2}{ }^{3} g+\frac{1}{2}{ }^{1} g=\overline{\mathrm{V}}^{\prime}-\frac{1}{4} \Delta^{\prime}, \\
& \text { with } \quad \overline{\mathrm{V}}^{\prime}=\frac{1}{4}{ }^{1} g+\frac{3}{4}{ }^{3} g \text { and } \Delta^{\prime}={ }^{3} g-{ }^{1} g,
\end{aligned}
$$

where the prime on $\overline{\mathrm{V}}^{\prime}$ and $\Delta^{\prime}$ is used to denote the central average and spin-spin matrix elements of the $\Lambda$ $\Sigma$ coupling interaction. Taking round numbers derived using the 10-range Gaussian interaction of Akaishi et al. (2000) that represents NSC97f yields ${ }^{3} g=4.8 \mathrm{MeV}$ and ${ }^{1} g=-1.0 \mathrm{MeV}$, which give $\overline{\mathrm{V}}^{\prime}=3.35 \mathrm{MeV}$ and $\Delta^{\prime}=5.8 \mathrm{MeV}$. Then, $v(0)=7.7 \mathrm{MeV}$ and $v(1)=1.9 \mathrm{MeV}$. In a simple $2 \times 2$ problem, the energy shifts of the $\Lambda$-hypernuclear states are given by $\sim v(J)^{2} / \Delta E$ with $\Delta E \sim 80 \mathrm{MeV}$ [and the admixture $\beta \sim-v(J) / \Delta E$ ]. Thus, the downward energy shift for the $0^{+}$state is $\sim 0.74 \mathrm{MeV}$ while the shift for the $1^{+}$state is small. The result is close to that for the NSC97f interaction in Fig. 1 of Akaishi et al. (2000). 
The observed CSB in the $A=4$ system is partially due to differences in Coulomb energies of the core nuclei, and to the mass difference between $\Sigma^{ \pm}$which is $\approx 10 \%$ of the $\Lambda-\Sigma$ mass difference, but the fundamental CSB in the $\Lambda N$ interaction is significant, and associated primarily with electromagnetic $\Lambda-\Sigma^{0}$ mixing that breaks isospin (Dalitz and von Hippel, 1964a). The CSB of the excited states differs from that of the ground states, and obtaining the correct level splittings is not trivial. As with the case of the hypertriton discussed above, CSB constrains the in-medium $\Lambda N$ interaction, in particular the stronginteraction coupling of $\Lambda$ 's and $\Sigma$ 's in the hypernuclear wavefunction (Gibson and Lehman, 1979; Akaishi et al., 2000; Hiyama et al., 2001; Nemura, Akaishi, and Suzuki 2002; Nogga, Kamada, and Glöckle, 2002; Nogga, 2013; Gal, 2015).

Faddeev-Yakubovsky studies (Nogga, Kamada, and Glöckle, 2002; Nogga, 2013) of the $A=4$ hypernuclei using the Nijmegen NSC97 (Rijken, Stoks, and Yamamoto, 1999) soft-core $Y N$ potentials fail to reproduce the isodoublet CSB splittings, although NSC97f, in particular, does a good job of reproducing the binding energy of ${ }_{\Lambda}^{3} \mathrm{H}$ and the $0^{+}-1^{+}$excitation energy in the $A=4$ hypernuclei. The same study also concludes that the probability of finding a $\Sigma$ in the $A=4$ hypernuclei is about $1.5 \%$, depending on the potential. Higher probability, of order $4 \%$ results by using NSC89 (Maessen, Rijken, and de Swart, 1989), and the CSB it yields is much larger than for NSC97, but NSC89 is definitely not a realistic YN potential for use in hypernuclei. Likewise, the Jülich'04 interaction (Haidenbauer and Meißner, 2005) is unsuitable (Nogga, 2013; Gazda et al., 2014; Wirth et al., 2014), especially in its $\Lambda-\Sigma$ coupling characteristics. However, the Jülich chiral $Y N$ model at LO (Polinder, Hedenbauer, and Meißner, 2006) shows promise (Nogga, 2013, Wirth et al. 2014), as does (Nogga, 2013) the NLO model (Haidenbauer et al., 2013).

The observation of $\pi^{+}$decay of ${ }_{\Lambda}^{4} \mathrm{He}$ (see Sec. II.B.1) supports the supposition that the wave function of this hypernucleus contains a non-negligible $\Sigma$ component, although the $\Sigma$ admixture required is considerably beyond those provided by the $A=4$ hypernuclear few-body calculations (Nemura, Akaishi, and Suzuki, 2002; Nogga, Kamada, and Glöckle, 2002; Nogga, 2013).

Another few-body, variational calculation (Nemura, Akaishi, and Suzuki, 2002) attempted to explicitly include three-body forces within a coupled-channel approach. This study claims to have obtained reasonable agreement with the separation energies for all the $s$-shell hypernuclei, including the excited states, by using a NSC97e-simulated potential. However, the genuine NSC97e potential in Nogga's calculation (Nogga, Kamada, and Glöckle, 2002; Nogga, 2013) significantly underbinds ${ }_{\Lambda}^{3} \mathrm{H}$. Therefore, there appears sufficient discrepancy between the results of theoretical calculations, and also when compared to the data, to warrant a more conservative view that all calculations are still missing something.

To summarize the status of $a b$ initio calculations, the $\Lambda N$ interaction is weaker than the $N N$ interaction, in part because one-pion exchange between a $\Lambda$ and a nucleon is forbidden by isospin. The inclusion of two-pion exchange introduces coupling of $\Lambda$ 's and $\Sigma$ 's in hypernuclei, in analogy to the coupling of $\Delta$ 's with nucleons in nuclei. However, $\Lambda-\Sigma$ coupling is much more important because of the suppression of the long-range OPE and the smaller mass difference between the $\Lambda$ and $\Sigma$. $\Lambda-\Sigma$ coupling naturally induces three-body forces as generated by the last diagram in Fig. 14 (Nemura, Akaishi, and Suzuki, 2002), and electromagnetic $\Lambda-\Sigma^{0}$ mixing generates charge-symmetry breaking Gal, 2015, Gazda and Gal, 2016). Thus the use of a $\Lambda N$ potential in a many-body calculation must include in-medium effects, as these are not included in any two-body "elementary" potential (Nemura, Akaishi, and Suzuki, 2002; Nogga, Kamada, and Glöckle, 2002).

\section{2. $p$-shell hypernuclei, $\gamma$-ray measurements, and spin dependence of the $\Lambda N$ interaction}

The results from various production reactions for hypernuclei have establishd that the $\Lambda$ moves in a potential well about $30 \mathrm{MeV}$ deep and that the $\boldsymbol{l}_{N \Lambda} \cdot \boldsymbol{s}_{\Lambda}$ spin-orbit term is quite small. However, multiplets based on particular core levels cannot be resolved. The splitting of a multiplet is governed by terms in Eq. (10) that depend on the spin of the $\Lambda$. In the $p$ shell, the five $p_{N} s_{\Lambda}$ two-body matrix elements depend on the radial integrals associated with each component in Eq. 10, are conventionally denoted by the parameters $\bar{V}, \Delta, S_{\Lambda}, S_{N}$ and $T$ (Gal, Soper, and Dalitz, 1971)

$$
V_{\Lambda N}=\bar{V}+\Delta \boldsymbol{s}_{N} \cdot \boldsymbol{s}_{\Lambda}+S_{\Lambda} \boldsymbol{l}_{N} \cdot \boldsymbol{s}_{\Lambda}+S_{N} \boldsymbol{l}_{N} \cdot \boldsymbol{s}_{N}+T S_{12}
$$

Note that the operators associated with $\Delta$ and $S_{\Lambda}$ are $\boldsymbol{S}_{N} \cdot \boldsymbol{s}_{\Lambda}$ and $\boldsymbol{L}_{N} \cdot \boldsymbol{s}_{\Lambda}$ with $\boldsymbol{S}_{N}$ and $\boldsymbol{L}_{N}$ the total nuclear Pauli spin and the total orbital angular momentum, respectively. This enables simple estimates for the contributions of $\Delta$ and $S_{\Lambda}$ to be made from the known $L S$ structure of the nuclear-core state.

The only way to measure the doublet spacings, and hence determine $\Delta, S_{\Lambda}$, and $T$, is to perform $\gamma$-ray spectroscopy with high-resolution $\gamma$-ray detectors. Figure 16 shows $20 \gamma$-ray transitions observed in $p$-shell hypernuclei via $\left(\pi^{+}, K^{+} \gamma\right)$ experiments at KEK and $\left(K^{-}, \pi^{-} \gamma\right)$ experiments at BNL between 1998 and 2005 using the Hyperball array of 14 large-volume Ge detectors (Hashimoto and Tamura, 2006). It can be seen that the data set includes the measurement of nine doublet spacings. As will be discussed, the data for ${ }_{\Lambda}^{7} \mathrm{Li},{ }_{\Lambda}^{9} \mathrm{Be}$, and ${ }_{\Lambda}^{16} \mathrm{O}$ play an important role in determining $\Delta, S_{\Lambda}$, and $T$, respectively. Also, looking ahead, Table VIII shows that all 


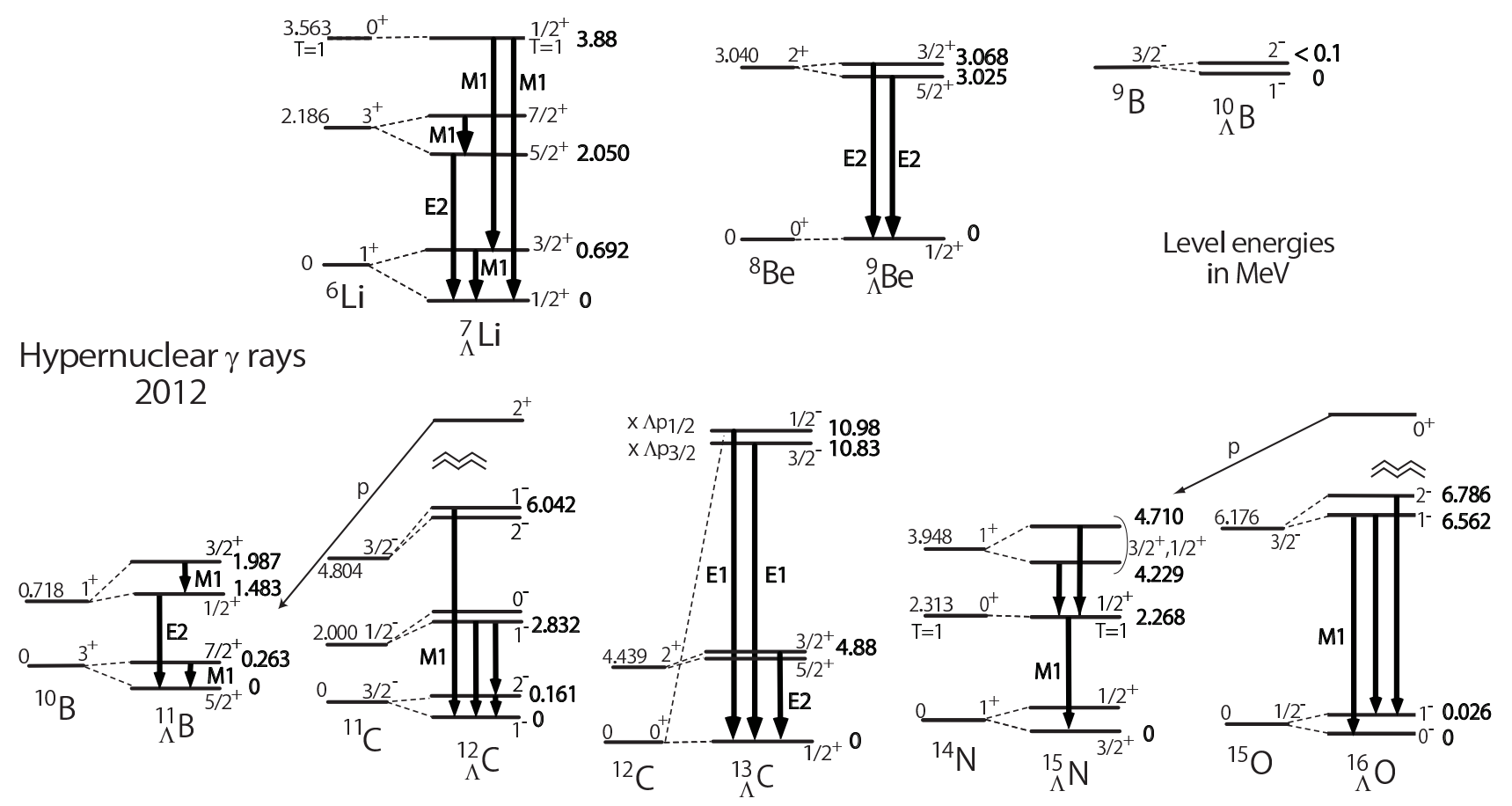

FIG. 16 Spectra of $p$-shell hypernuclei showing observed $\gamma$-ray transitions, all with the Hyperball detector except for the transitions in ${ }_{\Lambda}^{13} \mathrm{C}\left(\right.$ Ajimura et al. 2001 , Kohri et al., 2002) and ${ }_{\Lambda}^{12} \mathrm{C}$, for which the Hyperball2 detector was used (Hosomi et al. 2015). All energies are in MeV. Adapted from Tamura et al., 2013.

TABLE VIII Doublet spacings in $p$-shell hypernuclei. $E_{c}$ identifies the core state upon which the doublet is built. Energies are given in $\mathrm{keV}$. The entries in the top (bottom) half of the table are calculated using the parameters in Eq. 23] [Eq. 24]]. The individual contributions do not sum to exactly $\Delta E^{t h}$, which comes from the diagonalization, because small contributions from the energies of admixed core states are not included.

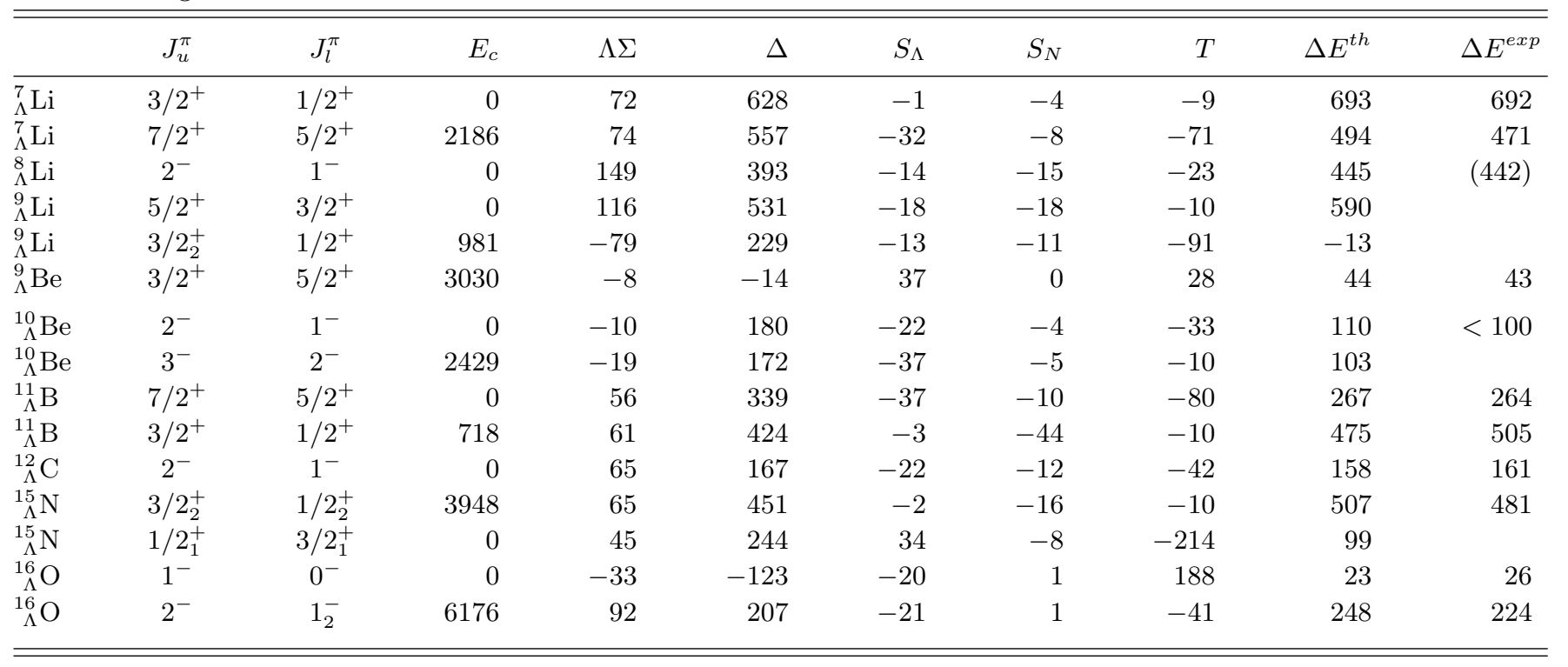

nine doublet spacings can be well described in terms of the contributions of these three parameters and contributions arising from $\Lambda-\Sigma$ mixing.

The motivation for including both $\Lambda$ and $\Sigma$ hypernuclear states in the shell-model basis is provided in the previous subsection where it is noted that the coupling between these configurations is necessary to solve the "overbinding" problem in the $s$-shell hypernuclei by providing considerable extra binding energy for the ${ }_{\Lambda}^{4} \mathrm{H}$ and ${ }_{\Lambda}^{4} \mathrm{He} 0^{+}$ground states. This means that the $\Lambda N$ spin-spin interaction and $\Lambda-\Sigma$ coupling both contribute strongly to the spacing of the $0^{+}$and $1^{+}$states. 
The $s_{N} s_{Y}$ matrix elements depend entirely on relative $s$ states while the central $p_{N} s_{Y}$ matrix elements come from roughly half relative $s$ state and half relative $p$ state. Because the $p$-state matrix elements are much smaller than $s$-state matrix elements, the scale for energy shifts from $\Lambda-\Sigma$ coupling desreases by factor of four in $p$-shell hypernuclei. This can be seen from Fig. 16 and Table VIII but the effects are still significant.

The parametrization of Eq. (18) applies to the direct $\Lambda N$ interaction, the $\Lambda N-\Sigma N$ coupling interaction, and the direct $\Sigma N$ interaction for both isospin $1 / 2$ and $3 / 2$. Thus, the input to shell-model calculations is four sets of the five parameters defined by Eq. [18). The parameter values of most interest are those for the $\Lambda N$ and $\Lambda N-\Sigma N$ interactions and a prime indicates the $\Lambda N-\Sigma N$ parameters. Values for these two parameter sets are based on various Nijmegen models of the $Y N$ interactions are given in Section III of Millener (2010); see also Yamamoto, Motoba, and Rijken (2010). The central interactions given by $\bar{V}^{\prime}$ and $\Delta^{\prime}$ are dominant for the $\Lambda N$ $\Sigma N$ interaction. To see which nuclear core states contribute to the $\Lambda-\Sigma$ coupling, and make contact with the approach of Umeya and Harada $(2009,2011)$, one can include an overall factor $\sqrt{4 / 3} t_{N} \cdot \boldsymbol{t}_{\Lambda \Sigma}$ that has a value of unity for the two-body matrix elements in Eq. (18), where $\boldsymbol{t}_{\Lambda \Sigma}$ is the operator that converts a $\Lambda$ into a $\Sigma$. Then, the core operator associated with $\bar{V}^{\prime}$ is $\boldsymbol{T}_{N}=\sum_{i} \boldsymbol{t}_{N i}$. This leads to a non-zero matrix element only between $\Lambda$ and $\Sigma$ states that have the same core, with the value

$$
\left\langle\left(J_{c} T, s_{\Sigma}\right) J T\left|V_{\Lambda \Sigma}\right|\left(J_{c} T, s_{\Lambda}\right) J T\right\rangle=\sqrt{\frac{4}{3}} \sqrt{T(T+1)} \bar{V}^{\prime},
$$

in analogy to Fermi $\beta$ decay of the core nucleus. Similarly, the spin-spin term involves $\sum_{i} \boldsymbol{s}_{N i} \boldsymbol{t}_{N i}$ for the core and connects core states that have large Gamow-Teller (GT) matrix elements between them. This can be the case when the core states are the same [this has been called coherent $\Lambda-\Sigma$ coupling (Akaishi et al. , 2000)] but, because $\Delta^{\prime}$ is large, there can be large coupling matrix elements for other states, often with different isospin but with the same spatial symmetry. Not surprisingly, energy shifts due to $\Lambda-\Sigma$ coupling grow with the isospin of the core nucleus and are predicted to be more than 250 $\mathrm{keV}$ for the ground states of ${ }_{\Lambda}^{9} \mathrm{He}$ and ${ }_{\Lambda}^{10} \mathrm{Li}$ that could be reached by double-charge-exchange reactions from stable targets (Gal and Millener, 2013).

Shell-model calculations for $p$-shell hypernuclei start with the Hamiltonian

$$
H=H_{N}+H_{Y}+V_{N Y},
$$

where $H_{N}$ is an empirical Hamiltonian for the $p$-shell core, the single-particle $H_{Y}$ supplies the $\sim 80 \mathrm{MeV}$ mass difference between $\Lambda$ and $\Sigma$, and $V_{N Y}$ is the $Y N$ interaction. The shell-model basis states are chosen to be of the form $\left|\left(p^{n} \alpha_{c} J_{c} T_{c}, j_{Y} t_{Y}\right) J T\right\rangle$, where the hyperon is coupled in angular momentum and isospin to eigenstates of the $p$-shell Hamiltonian for the core, with up to three values of $T_{c}$ contributing for $\Sigma$-hypernuclear states. This is known as a weak-coupling basis and, indeed, the mixing of basis states in the hypernuclear eigenstates is generally very small. In this basis, the core energies can be taken from experiment where possible and from the $p$-shell calculation otherwise.

The technical details of such calculations are quite simple (Auerbach et al., 1983, Millener, 2007). Because the product of creation and annilation operators for a twobody $Y N$ interaction can written in terms $a^{\dagger} a$ pairs for the nucleons and hyperons, we simply need a complete set of OBDME between $p$-shell eigenstates (the maximum dimension for a given $J T$ in the $p$ shell is only 14) to compute matrix elements of the hypernuclear Hamiltonian. Only isoscalar OBDME are needed in the $\Lambda$ space and isovector OBDME are needed for the $\Lambda-\Sigma$ coupling matrix elements.

Many hypernuclear calculations have used the venerable Cohen and Kurath (1965) interactions. Here, the $p$-shell interaction has been refined using the following strategy. The one-body spin-orbit splitting between the $p_{3 / 2}$ and $p_{1 / 2}$ orbits is fixed to give a good description of the light $p$-shell nuclei (say for $A \leq 9$ ). The overall strength of the tensor interaction is also fixed, ultimately to produce the cancellation in ${ }^{14} \mathrm{C} \beta$ decay. The well-determined linear combinations of the central and vector $p$-shell interactions are then chosen by fitting the energies of a large number of states that are known to be dominantly $p$ shell in character, including the large spin-orbit splitting at $A=15$. Some properties of stable $p$-shell ground states are shown in Table IX for this interaction in the supermultiplet basis where $[f] K_{L} L$ label representations of $\mathrm{SU}(3) \supset \mathrm{R} 3$ in the orbital space (three single-particle $p$ states) and $[\widetilde{f}] \beta T S$ label representations of $\mathrm{SU}(4) \supset \mathrm{SU}(2) \times \mathrm{SU}(2)$ in the spin-isospin space (four states); $[f]=\left[f_{1} f_{2} f_{3}\right]$, with $f_{1} \geq f_{2} \geq f_{3}$ and $f_{1}+f_{2}+f_{3}=n$, also labels the spatial symmetry. $K_{L}$ labels multiple occurrences of $L$ for a given representation of $\mathrm{SU}(3)$ and is obtained by angular-momentum projection from a specific intrinsic $\mathrm{SU}(3) \supset \mathrm{SU}(2)$ basis state; when $S \neq 0, J$ can be projected from a product of the $\mathrm{SU}(3) \supset \mathrm{SU}(2)$ intrinsic state and an intrinsic spin state with magnetic quantum number $K_{S}$ to give a state with $K=K_{L}+K_{S}$ and a mixture of $L$ values [see Eqs. 21] and (22)]. The central interaction is essentially SU(4) conserving and the mixing of different $\left[f_{c}\right] L_{c} S_{c}$ is primarily due to the one-body spin-orbit and two-body SLS and ALS terms in the effective $p$-shell Hamiltonian. A detailed discussion of $p$-shell nuclei, including the allowed quantum numbers and spectra, is given in Section 5 of Millener (2007). In Table IX

$$
|K=J=3 / 2\rangle=\sqrt{\frac{21}{26}}|L=1\rangle-\sqrt{\frac{5}{26}}|L=2\rangle
$$


TABLE IX Root-mean-square charge radii and dominant wave function components for the ground states of stable $p$ shell nuclei (par4 interaction). $[f]$ labels the spatial symmetry of the $p$-shell nucleons (see text). The $L$ decomposition of states with good $K$ are given in Eqs. (21) and (22).

\begin{tabular}{ccrcll}
\hline \hline Nucleus & $\left\langle r^{2}\right\rangle_{c h}^{1 / 2} \mathrm{fm}$ & {$[f]$} & $\%[f]$ & $J^{\pi}$ & Dominant component \\
\hline${ }^{6} \mathrm{Li}$ & 2.57 & {$[2]$} & 98.2 & $1^{+}$ & $L=0, S=1$ \\
${ }^{7} \mathrm{Li}$ & 2.41 & {$[3]$} & 96.6 & $\overline{3}^{-}$ & $L=1, S=\frac{1}{2}$ \\
${ }^{9} \mathrm{Be}$ & 2.52 & {$[41]$} & 94.7 & $\overline{3}^{-}$ & $K=\frac{3}{2}, S=\frac{1}{2}$ \\
${ }^{10} \mathrm{~B}$ & 2.45 & {$[42]$} & 94.0 & $3^{+}$ & $K=3, S=1$ \\
${ }^{11} \mathrm{~B}$ & 2.42 & {$[43]$} & 81.0 & $\frac{3}{2}^{-}$ & $K=\frac{3}{2}, S=\frac{1}{2}$ \\
${ }^{12} \mathrm{C}$ & 2.47 & {$[44]$} & 79.3 & $0^{+}$ & $L=0, S=0$ \\
${ }^{13} \mathrm{C}$ & 2.44 & {$[441]$} & 66.5 & $\frac{1}{2}^{-}$ & $L=1, S=\frac{1}{2}$ \\
${ }^{14} \mathrm{C}$ & 2.56 & {$[442]$} & 59.7 & $0^{+}$ & $L=0, S=0$ \\
${ }^{14} \mathrm{~N}$ & 2.52 & {$[442]$} & 94.2 & $1^{+}$ & $L=2, S=1$ \\
${ }^{15} \mathrm{~N}$ & 2.59 & {$[443]$} & 100.0 & $\frac{1}{2}^{-}$ & $L=1, S=\frac{1}{2}$ \\
\hline \hline
\end{tabular}

with $S=1 / 2$, while

$$
|K=J=3\rangle=\sqrt{\frac{6}{7}}|L=2\rangle-\sqrt{\frac{3}{22}}|L=3\rangle+\sqrt{\frac{1}{154}}|L=4\rangle,
$$

with $S=1$.

In the LS basis for the core, the matrix elements of $\boldsymbol{S}_{N} \cdot \boldsymbol{s}_{\Lambda}$ are diagonal [similarly for $\boldsymbol{L}_{N} \cdot \boldsymbol{s}_{\Lambda}=\left(\boldsymbol{J}_{N}-\boldsymbol{S}_{N}\right) \cdot \boldsymbol{s}_{\Lambda}$ ] and depend just on the intensities of the total $L$ and $S$ for the hypernucleus. Because supermultiplet symmetry $\left[f_{c}\right] K_{c} L_{c} S_{c} J_{c} T_{c}$ is generally a good symmetry for $p$-shell core states [Table IX and Eqs. (21) and [22]], only one or two values of $L$ and $S$ are important. Of the remaining $\Lambda N$ parameters, $\bar{V}$ contributes only to the overall binding energy; $S_{N}$ does not contribute to doublet splittings in the weak-coupling limit but a negative $S_{N}$ augments the nuclear spin-orbit interaction and contributes to the spacings between states based on different core states; in general, there are no simple expressions for the coefficients of $T$.

With reference to Table VIII, the set of $\Lambda N$ parameters used up to ${ }_{\Lambda}^{9} \mathrm{Be}$ (chosen to fit the energy spacings in ${ }_{\Lambda}^{7} \mathrm{Li}$ perfectly) is (parameters in $\mathrm{MeV}$ )

$$
\Delta=0.430 S_{\Lambda}=-0.015 S_{N}=-0.390 T=0.030 .
$$

The doublet spacings for the heavier $p$-shell hypernuclei consistently require a smaller value for $\Delta$

$$
\Delta=0.330 \quad S_{\Lambda}=-0.015 \quad S_{N}=-0.350 \quad T=0.0239 .
$$

The matrix elements for the $\Lambda-\Sigma$ coupling interaction, based on the G-matrix calculations of Akaishi et al. (2000) for the NSC97e, $f$ interactions (Rijken, Stoks, and Yamamoto, 1999), are

$$
\bar{V}^{\prime}=1.45 \quad \Delta^{\prime}=3.04 \quad S_{\Lambda}^{\prime}=S_{N}^{\prime}=-0.09 \quad T^{\prime}=0.16 .
$$

These parameters are kept fixed throughout the $p$ shell.
We are now in a position to consider the $\gamma$-ray data in Fig. 16 in relation to the breakdown of doublet spacings in Table VIII. First, on a historical note, shell-model analyses of $\Lambda$ binding energies for $p$-shell hypernuclei were attempted long ago, and introduced the notation still in use for the $\Lambda N$ interaction (Gal, Soper, and Dalitz, 1971). They also considered a double one-pion-exchange $\Lambda N N$ interaction. However, progress on characterizing the $\Lambda N$ interaction was hampered by a lack of data (Gal, Soper, and Dalitz, 1972, 1978). Nevertheless, the stage was set for studies of hypernuclear $\gamma$-rays (Dalitz and Gal, 1978). The observation of $\gamma$-rays in ${ }_{\Lambda}^{7} \mathrm{Li}$ and ${ }_{\Lambda}^{9} \mathrm{Be}$ at BNL using the $\left(K^{-}, \pi^{-} \gamma\right)$ reaction and NaI detectors (May et al. 1983) finally permitted a convincing shell-model analysis (Millener et al. . 1985) with parameters close to those in Eq. (23), but without the inclusion of $\Lambda-\Sigma$ coupling, and inspired other analyses (Fetisov et al., 1991). Many of the $p$-shell hypernuclei up to ${ }_{\Lambda}^{13} \mathrm{C}$ have also been studied in cluster models (Hiyama and Yamada, 2009).

In the first $\left(\pi^{+}, K^{+} \gamma\right)$ experiment with the Hyperball at KEK in 1998 (Tamura et al. 2000), four $\gamma$-rays in ${ }_{\Lambda}^{7} \mathrm{Li}$ were seen, namely all except the $7 / 2^{+} \rightarrow 5 / 2^{+}$transiton in Fig. 16. Note that the $3 / 2^{+}(L=0, S=3 / 2)$ and $7 / 2^{+}$ $(L=2, S=3 / 2)$ require spin-flip and are not strongly populated in the $\left(\pi^{+}, K^{+}\right)$reaction (Hiyama et al. 1999$)$. The high-energy M1 transitions from the $1 / 2^{+} ; T=1$ level can be seen when the Doppler-shift correction is made and their energy difference matches the $691.7 \mathrm{keV}$ of the transition (peak sharpened by the Doppler correction) between the ground-state doublet members. The lineshape for the $2050-\mathrm{keV} 5 / 2^{+} \rightarrow 1 / 2^{+}$transition gives a lifetime for the $5 / 2^{+}$level via the Doppler-shift attenuation method (Tanida et al. 2001). The derived reduced electric-quadrupole transition probability $\mathrm{B}(\mathrm{E} 2)$ is considerably smaller than expected from the known $\mathrm{B}(\mathrm{E} 2)$ for the $3^{+} \rightarrow 1^{+}$transition in ${ }^{6} \mathrm{Li}$. The lowest threshold is for ${ }_{\Lambda}^{5} \mathrm{He}+d$ at 3.94(4) $\mathrm{MeV}$ so that the $5 / 2^{+}$state and the $1 / 2^{+}$ground state in ${ }_{\Lambda}^{7} \mathrm{Li}$ are considerably more bound than the core states in ${ }^{6} \mathrm{Li}$. This entails a shrinkage in the size of the radial wave functions, and a reduction of the $\mathrm{B}(\mathrm{E} 2)$, that is best treated in cluster-model calculations for ${ }_{\Lambda}^{7} \mathrm{Li}$ (Hiyama et al., 1999). The 471-keV M1 $\gamma$-ray in the upper doublet was seen via $\gamma-\gamma$ coincidence with the $5 / 2^{+} \rightarrow 1 / 2^{+}$transition in a $\left(K^{-}, \pi^{-} \gamma\right)$ experiment on a ${ }^{10} \mathrm{~B}$ target at BNL (Ukai et al. 2006) (following $l=0{ }^{3} \mathrm{He}$ emission from the $s_{N}^{-1} s_{\Lambda}$ substitutional state in ${ }_{\Lambda}^{10} \mathrm{~B}$ ).

From Table VIII it can be seen that the ${ }_{\Lambda}^{7} \mathrm{Li}$ groundstate doublet spacing comes mostly from the spin-spin interaction $\left(\frac{3}{2} \Delta\right.$ in the pure LS limit) with a $10 \%$ assistance from $\Lambda-\Sigma$ coupling. The situation is similar for the second doublet except that contributions from $S_{\Lambda}$ and $T$ reduce the spacing by $\sim 100 \mathrm{keV} . S_{N}$ reduces the excitation energies of the $5 / 2^{+} ; 0$ and $1 / 2^{+} ; 1$ states by 288 $\mathrm{keV}$ and $82 \mathrm{keV}$, respectively (Millener, 2007), making the $1 / 2^{+}$state just bound.

In ${ }_{\Lambda}^{9} \mathrm{Be}$, the ${ }^{8} \mathrm{Be}$ core states are unbound (by $92 \mathrm{keV}$ 
for the ground state) but the presence of the $\Lambda$ raises the $\alpha$ threshold to $3.50 \mathrm{MeV}$, viz.

$$
B_{\alpha}\left({ }_{\Lambda}^{9} \mathrm{Be}\right)=B_{\alpha}\left({ }^{8} \mathrm{Be}\right)+B_{\Lambda}\left({ }_{\Lambda}^{9} \mathrm{Be}\right)-B_{\Lambda}\left({ }_{\Lambda}^{5} \mathrm{He}\right),
$$

meaning that the $\gamma$-rays from the $3 / 2^{+}$and $5 / 2^{+}$states can be observed. This was achieved using the Hyperball in a $\left(K^{-}, \pi^{-} \gamma\right)$ experiment at BNL (Akikawa et al. 2002). With the Doppler correction, peaks were seen at 3024 and $3067 \mathrm{keV}$ [these are updated energies (Tamura, 2010)]. Only the upper peak is seen following proton emission from ${ }_{\Lambda}^{10} \mathrm{~B}$ and strong theoretical arguments Millener, 2005, 2007) indicate that this $\gamma$-ray comes from the $3 / 2^{+}$member of the doublet. Table VIII shows that the small splitting of the doublet means that $S_{\Lambda}$ is small (contributions from $\Delta, T$, and $\Lambda-\Sigma$ coupling more or less cancel); the splitting is $-\frac{5}{2} S_{\Lambda}$ if the ${ }^{8} \mathrm{Be} 2^{+}$state is pure $L=2, S=0$, as it is in the $2 \alpha+\Lambda$ cluster model (Hiyama et al. 2000).

An earlier experiment with NaI detectors at BNL (May et al. 1983) observed a $\gamma$-ray at $3079(40) \mathrm{keV}$ and put an upper limit of $100 \mathrm{keV}$ on the doublet splitting. This, and the observation of a $2034(23) \mathrm{keV} \gamma$-ray in ${ }_{\Lambda}^{7} \mathrm{Li}$ (May et al. 1983), revived shell-model studies of $p$-shell hypernuclei (Millener et al., 1985).

The main objective of a $2001\left(K^{-}, \pi^{-} \gamma\right)$ experiment at BNL (Ukai et al. 2004, 2008) was to measure the groundstate doublet spacing of ${ }_{\Lambda}^{16} \mathrm{O}$ that depends strongly on the matrix element of the $\Lambda N$ tensor interaction $T$. For a pure $p_{1 / 2}^{-1} s_{\Lambda}$ configuration, the spacing is Dalitz and Gal, 1978)

$$
E\left(1_{1}^{-}\right)-E\left(0^{-}\right)=-\frac{1}{3} \Delta+\frac{4}{3} S_{\Lambda}+8 T .
$$

Figure 16 shows that the measured spacing is only 26 $\mathrm{keV}$, derived from the difference in energies of the $\gamma$-rays from the $6562-\mathrm{keV}^{-}$excited state to the members of the ground-state doublet. Table VIII shows that the small separation is the result of a large cancellation between the contributions of $T$ and the other contributions (mainly $\Delta)$. If $\Delta$ is known, this doublet spacing fixes $T$. The major contributor to the increase in the spacing between the two doublets relative to the core spacing of $6.176 \mathrm{MeV}$ is $S_{N}$ which gives over $500 \mathrm{keV}\left(\sim-\frac{3}{2} S_{N}\right)$.

A weak $\gamma$-ray is also seen in the above experiment (Ukai et al., 2008) and is interpreted as a transition from the $2^{-}$member of the upper doublet [the $2^{-}$state requires spin-flip to be populated via the $\left(K^{-}, \pi^{-}\right)$reaction]. The ${ }_{\Lambda}^{15} \mathrm{~N} \gamma$-rays are seen following proton emission from the $p_{\Lambda}$ states of ${ }_{\Lambda}^{16} \mathrm{O}$ (see Fig. 17). The $2268-\mathrm{keV} \gamma$-ray is sharp without Doppler correction implying a long lifetime [measured at $1.5(4) \mathrm{ps}$ ] while the transitions from the upper doublet are fast and are seen when the Doppler correction is made. It is interesting that the transition from the $1 / 2^{+} ; 1$ level to the $1 / 2^{+}$member of the ground-state doublet is not seen; in the weak-coupling limit, it should

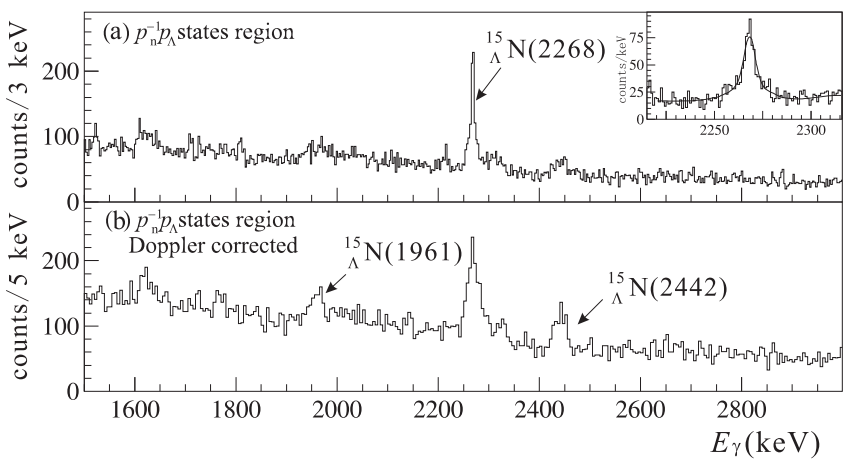

FIG. $17 \gamma$-ray spectra from ${ }_{\Lambda}^{15} \mathrm{~N}$ (see Fig. 16) following proton emission from the $p_{n}^{-1} p_{\Lambda}$ mass region of ${ }_{\Lambda}^{16} \mathrm{O}$ populated in the $\left(K^{-}, \pi^{-}\right)$reaction on ${ }^{16} \mathrm{O}$ (see Fig. 5). The upper figure shows that the $2268-\mathrm{keV}$ line is sharp without Doppler correction implying a long lifetime that is obtained from the lineshape analysis shown in the inset. The lower figure shows the transitions from the upper doublet that appear when the Doppler correction is made. Adapted from Ukai et al., 2008.

be approximately half the strength of the $2268-\mathrm{keV}$ transition. We first note that in ${ }^{14} \mathrm{~N}$ the M1 transition from the $3.498-\mathrm{MeV} 1^{+}$level (mainly $L=0, S=1$ ) to the $0^{+} ; 1$ level is strong while the M1 transition from the $0^{+} ; 1$ level to the ground-state is weak because this transtion is the analog of ${ }^{14} \mathrm{C} \beta$ decay and the $\langle\sigma \tau\rangle$ matrix element essentially vanishes (making the M1 transition mainly orbital). It turns out (Millener, 2007, Ukai et al. 2008 ) that small admixtures of the $1_{2}^{+} ; 0 \times s_{\Lambda}$ configuration into the wave functions of the ground-state doublet members produce strong cancellations in the hypernuclear M1 matrix elements giving a predicted lifetime of $0.5 \mathrm{ps}$ for the $0^{+} ; 1$ level compared with $0.1 \mathrm{ps}$ for the core transition. The cancellation is more severe for the $1 / 2^{+} ; 1 \rightarrow 1 / 2^{+}$ transition but still not quite strong enough because the calculated $\gamma$-ray branch to the $1 / 2^{+}$state is $18 \%$ while the experiment puts an upper limit of $\sim 5 \%$ at the predicted energy (Ukai et al., 2008). The upper doublet (the lower member is surely $1 / 2^{+}$) is based on an $L=0, S=1$ core and the splitting is mainly due to the spin-spin interaction $(\Delta)$ in analogy to the ${ }_{\Lambda}^{7} \mathrm{Li}$ ground-state doublet and, in fact, the first-excited-state doublet in ${ }_{\Lambda}^{11} \mathrm{~B}$.

In ${ }_{\Lambda}^{12} \mathrm{C}$, the excitation energies of the excited $1^{-}$states provide a useful check on the energies of the unresolved peaks in the ${ }^{12} \mathrm{C}\left(e, e^{\prime} K^{+}\right){ }_{\Lambda}^{12} \mathrm{~B}$ reaction (Iodice et al. 2007; Tang et al. (2014). The difference in the energies of the transitions from the $1_{2}^{-}$level agrees with the 161.5 $\mathrm{keV}$ energy measured for the ground-state doublet transition (Hosomi et al., 2015). This doublet spacing is important because of the failure to observe the corresponding doublet spacing in ${ }_{\Lambda}^{10} \mathrm{~B}$ in two $\left(K^{-}, \pi^{-} \gamma\right)$ experiments at BNL (Chrien et al., 1990, Ukai, 2004) that both set an upper limit of about $100 \mathrm{keV}$ on the doublet spacing. The core nuclei have similar structures (see Table IX], being essentially particle-hole conjugates in the $p$ shell (a par- 
ticle or hole in the Nilsson $K=3 / 2$ orbit). This means that the $\Lambda N$ contribution to the spacing should be nearly the same. TableVIII shows that the $\Lambda N$ contribution for ${ }_{\Lambda}^{10} \mathrm{~B}$ is actually slightly larger than for ${ }_{\Lambda}^{12} \mathrm{C}$. Table VIII also shows that the $\Lambda-\Sigma$ coupling increases the doublet separation in ${ }_{\Lambda}^{12} \mathrm{C}$ while decreasing it slightly in ${ }_{\Lambda}^{10} \mathrm{~B}$. This is because the $\langle\sigma \tau\rangle$ matrix elements involving the lowest $3 / 2^{-}$and $1 / 2^{-}$states are of opposite sign for the two core nuclei. The coefficients of $\bar{V}^{\prime}$ and $\Delta^{\prime}$ for matrix elements involving the same core state are of opposite sign for the $1^{-}$and $2^{-}$states and the sign changes between ${ }_{\Lambda}^{10} \mathrm{~B}$ and ${ }_{\Lambda}^{12} \mathrm{C}$. Although this is a substantial effect, it is lessened by that fact that the $1^{-}$states in both hypernuclei are pushed down by a coupling to $\Sigma$ states that have a $1 / 2^{-}$ core state. It is certainly possible to reduce the spacing in ${ }_{\Lambda}^{10} \mathrm{~B}$ appreciably by changing the $\Lambda-\Sigma$ coupling interaction (Halderson, 2008; Millener, 2010). It has also been suggested that charge-symmetry breaking effects could lower the transition energy in ${ }_{\Lambda}^{10} \mathrm{~B}$ (Gal, 2015).

Another way to try to measure the ground-state doublet spacing for the $A=10$ hypernuclei is to look for $\gamma$-rays from the $2^{-}$and $3^{-}$states in ${ }_{\Lambda}^{10} \mathrm{Be}$ based on the $2.43-\mathrm{MeV} 5 / 2^{-}$state in ${ }^{9} \mathrm{Be}$ via the ${ }^{10} \mathrm{~B}\left(K^{-}, \pi^{0} \gamma\right){ }_{\Lambda}^{10} \mathrm{Be}$ reaction (Millener, 2012); this reference also considers ${ }_{\Lambda}^{8} \mathrm{Li}$ and ${ }_{\Lambda}^{9} \mathrm{Be}$ as possible sources of unassigned $p$-shell hypernuclear $\gamma$ rays. Unfortunately, the $2_{2}^{-} \rightarrow 2_{1}^{-} \gamma$-ray branch is predicted to be only $13 \%$ and the $2_{2}^{-} \rightarrow 1_{1}^{-}$ and $3_{1}^{-} \rightarrow 2_{1}^{-}$transitions could have very similar energies. There is no chance to see the ground-state doublet transition itself because the $\mathrm{B}(\mathrm{M} 1)$ is proportional to $\left(g_{c}-g_{\Lambda}\right)^{2}$ (Dalitz and Gal, 1978) $\left(g_{c}=-0.746\right.$, $\left.g_{\Lambda}=-1.226\right)$ leading to very long electromagnetic lifetime meaning that the $2^{-}$level will undergo weak decay.

In the $\left(\pi^{+}, K^{+} \gamma\right)$ reaction on ${ }^{11} \mathrm{~B}$, six $\gamma$-ray transitions with energies of 264, 458, 505, 570, 1483, and $2477 \mathrm{keV}$ have been identified as transitions in ${ }_{\Lambda}^{11} \mathrm{~B}$ (Miura, 2005). The 1483-keV transition is by far the most intense and is identified as coming from the $1 / 2^{+}$level based on the $718-\mathrm{keV} 1^{+} ; 0$ level of ${ }^{10} \mathrm{~B}$ and acts as a collection point for $\gamma$-rays from strongly populated $3 / 2^{+}$and $1 / 2^{+}$levels higher in the spectrum. A $3 / 2^{+} ; 1$ level based on the $5.16-\mathrm{MeV} 2^{+} ; 1$ level of ${ }^{10} \mathrm{~B}$ should be the strongest and the source of the $2477-\mathrm{keV} \gamma$-ray seen in the Dopplercorrected spectrum. By making use of the relative intensities and lifetime limits for these $\gamma$-rays a plausible decay scheme has been established by comparison with shellmodel calculations (Millener, 2008). Assignments for the lower part of the spectrum, shown in Fig. 16, have been confirmed from an analysis of the three $\gamma$-rays seen following proton emission from ${ }_{\Lambda}^{12} \mathrm{C}$ (Ma et al., 2010). The main failing of the shell-model calculation is that it does not produce high enough excitation energies for the ${ }_{\Lambda}^{11} \mathrm{~B}$ states based on the $1^{+} ; 0$ states of ${ }^{10} \mathrm{~B}$ at 0.72 and 2.15 $\mathrm{MeV}$ (Millener, 2010).

The preceding discussion shows that one set of $p_{N} s_{Y}$ parameters is quite successful in reproducing data on the doublet spacings in the $p$ shell (with some adjustment for $\left.{ }_{\Lambda}^{7} \mathrm{Li}\right)$. This statement refers to $\Delta, S_{\Lambda}, T$ and the $\Lambda-\Sigma$ coupling parameters. The parameter $S_{N}$ augments the nuclear spin-orbit interaction, gives a substantial contribution to $B_{\Lambda}$ values in the $p$ shell (Millener, 2010), and works in the right direction to reproduce the changes in spacing of doublet centroids from the spacing in the core nucleus. However, a considerably larger value of $S_{N}$ is required to reproduce the energies of excited-state doublets in ${ }_{\Lambda}^{11} \mathrm{~B},{ }_{\Lambda}^{12} \mathrm{C}$, and ${ }_{\Lambda}^{13} \mathrm{C}$. In terms of the $\Lambda N$ interaction alone, the small value for $S_{\Lambda}$ means that the strengths of the symmetric and antisymmetric spin-orbit interactions have to be very nearly equal. This is not the case for effective interactions derived from free-space $Y N$ models and nor is the value for $S_{N}$ large enough (Millener, 2010). However, the double one-pion-exchange $\Lambda N N$ interaction (Gal, Soper, and Dalitz, 1971) is independent of the $\Lambda$ spin and gives, when averaged over the $s_{\Lambda}$ wave function, an effective $N N$ interaction that operates in the nuclear core. This interaction contains an antisymmetric spin-orbit component that behaves rather like $S_{N}$ and has its largest effect beyond the middle of the $p$ shell (Gal, Soper, and Dalitz, 1971). It may, in fact, be responsible for much of the empirical value of $S_{N}$ and should be reintroduced into $p$-shell hypernuclear calculations.

In ${ }_{\Lambda}^{13} \mathrm{C}$, the $\Lambda$ threshold is the lowest particle-decay channel and the $p_{\Lambda}$ orbit is just bound. As noted earlier, the $\sim 11-\mathrm{MeV} \gamma$-rays from the lowest $3 / 2^{-}$and $1 / 2^{-}$ states were measured using an array of $\mathrm{NaI}$ detectors and the separation of the states, $152 \pm 54$ (stat) \pm 36 (syst), was determined from the shift in the peak with pion scattering angle (Ajimura et al., 2001, Kohri et al., 2002). Figure 18 shows the $p^{8} p_{\Lambda}$ states based on the lowest $0^{+}$and $2^{+}$states of the ${ }^{12} \mathrm{C}$ core. From an older BNL experiment (May et al. , 1983), the separation between the two $1 / 2^{-}$states was determined to be $6.0 \pm 0.4 \mathrm{MeV}$ while that of the $1 / 2_{2}^{-}$and $5 / 2_{2}^{-}$states was $1.7 \pm 0.4 \mathrm{MeV}$. The doublets are characterized by the quantum number $\mathcal{L}$ and split by the spin-dependent interactions where Auerbach et al. 1981, 1983)

$$
\mathcal{L}=\boldsymbol{J}_{c}+\boldsymbol{l}_{\Lambda} \quad \text { and } \quad \boldsymbol{J}=\mathcal{L}+\boldsymbol{s}_{\Lambda} .
$$

The spectrum, including $\Lambda-\Sigma$ coupling, can be calculated from the Gaussian or Yukawa representations of the $\mathrm{G}$ matrices derived from the free $Y N$ interaction model. Beause the $p_{\Lambda}$ states are only bound by about 0.8 $\mathrm{MeV}$, the calculation is performed using Woods-Saxon wave functions for this binding energy. One can also use an interaction obtained by adjusting the strengths in the various $\Lambda N$ channels to reproduce the $p_{N} s_{\Lambda}$ matrix elements in Eq. (24). There are 20 independent $p_{N} p_{\Lambda}$ matrix elements and pieces of the interactions such as the even-state tensor interaction enter. Furthermore, a $Q_{N} \cdot Q_{\Lambda}$ multipole component of the interaction is active as compared to just the spatial monopole for $p_{N} s_{\Lambda}$. It is this quadrupole component that splits the $\mathcal{L}=1,2,3$ 


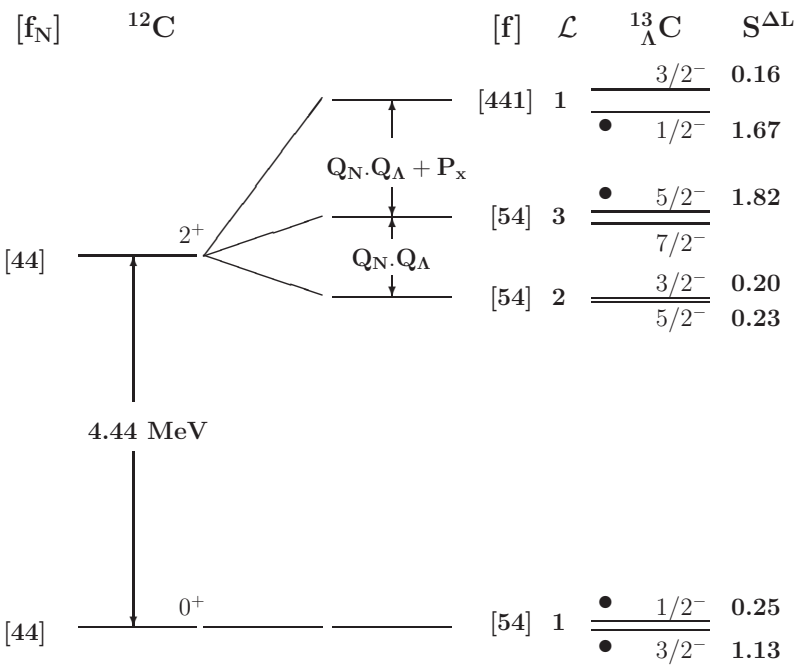

FIG. $18 p_{\Lambda}$ states in ${ }_{\Lambda}^{13} \mathrm{C}$ based on the lowest $0^{+}$and $2^{+}$ states of the ${ }^{12} \mathrm{C}$ core. The spin-doublet structure is explained in the text and Eq. 28). The states of the $2^{+} \times p_{\Lambda}$ multiplet are split by the quadrupole-quadrupole component of the $p_{N} p_{\Lambda}$ interaction. The states are labelled by their tendency towards a good supermultiplet symmetry $[f]$. The energy of the uppermost doublet is sensitive to the space-exchange component in the $\Lambda N$ interaction. The $S^{\Delta L}$ on the right are structure factors governing the relative population of states in the $\left(K^{-}, \pi^{-}\right)$reaction with no spin flip $(\Delta L=0$ for the $1 / 2^{-}$states and $\Delta L=2$ for the others).

states of the $2^{+} \times p_{\Lambda}$ multiplet in Fig. 18 . This can involve strong mixing of the $p_{1 / 2}$ and $p_{3 / 2} \Lambda$ states to make states with good $\mathcal{L}$ (Auerbach et al. 1983$)$.

For $p_{N} s_{\Lambda}$, there is no way to separate the contributions from the even- and odd-state central interactions. However, for $p_{N} p_{\Lambda}$ different strengths in the even- and oddstate central interactions give rise to a space-exchange interaction that will separate states with different spatial symmetries. Coupling a $p_{\Lambda}$ to the dominantly [44] states of ${ }^{12} \mathrm{C}$ leads to [54] and [441] symmetries for the nine $p$-shell baryons. These are not very good quantum numbers for the hypernuclear states. Nevertheless, the uppermost doublet in Fig. 18 tends towards [441] symmetry; note the large structure factor for the substitutional $1 / 2^{-}$ state reached via $\Delta L=0, \Delta S=0$ from the ${ }^{13} \mathrm{C}$ ground state in the $\left(K^{-}, \pi^{-}\right)$reaction. The excitation energy of this doublet is indeed sensitive to the space-exchange interaction. For example, the NSC97f interaction has repulsion in both singlet- and triplet-odd states producing a too large separation of $\sim 6.9 \mathrm{MeV}$ from the lower $\mathcal{L}=1$ doublet and a separation of $\sim 2.2 \mathrm{MeV}$ from the $\mathcal{L}=3$ doublet. On the other hand, the ESC04 model (Rijken and Yamamoto, 2006a) has repulsion in the singlet-odd channel and attraction in the triplet-odd channel giving 6.0 and $1.2 \mathrm{MeV}$ for the two separations. We note that the ${ }^{12} \mathrm{C}$ ground state has a considerable $L=1, S=1 \mathrm{com}$ ponent that allows various spin-dependent components of the $\Lambda N$ interaction to contribute to the spacing of the lowest $1 / 2^{-}$and $3 / 2^{-}$states, in contrast to the situation for the $3 \alpha+\Lambda$ model (Hiyama et al. 2000$)$. The tensor interaction and the $\Lambda-\Sigma$ coupling both work to put the $1 / 2^{-}$state below the $3 / 2^{-}$state.

The $p^{n} p_{\Lambda}$ shell-model calculations were performed (Auerbach et al. 1983$)$ to understand $\left(K^{-}, \pi^{-}\right)$ reaction data coming from CERN and BNL. While these calculations have been updated to include $\Lambda-\Sigma$ coupling and the use of realistic radial wave functions, they need to be extended to full $1 \hbar \omega$ calculations that include an $s_{\Lambda}$ coupled to $1 \hbar \omega$ states of the core nucleus. These states are mixed with the $p^{n} p_{\Lambda}$ states both by the $\Lambda N$ interaction and by the requirement that the physical $1 \hbar \omega$ states are free from spurious center-of-mass components. The need for such calculations is apparent in the extra structure near the $p_{\Lambda}$ peak in Fig. 10 and the fact that a number of $p$-shell hypernuclear $\gamma$-rays are seen in ${ }_{\Lambda}^{9} \mathrm{Be},{ }_{\Lambda}^{11} \mathrm{~B}$, and ${ }_{\Lambda}^{15} \mathrm{~N}$ following proton emission from the primary hypernucleus. In the latter case, the $p^{n-1}(s d) s_{\Lambda}$ component in the wave function gives the $(s d)$ proton spectroscopic factor that controls the relative population of states in the daughter hypernucleus.

The Nijmegen baryon-baryon interactions have continued to evolve with a variety of ESC04 (Rijken and Yamamoto, 2006a) and ESC08 (Rijken, Nagels, and Yamamoto, 2010; Nagels, Rijken, and Yamamoto, 2015b) models becoming available. The improvements cover many aspects from strangeness 0 to -4 (Rijken, Nagels, and Yamamoto, 2013). As far as $p$-shell spectra are concerned, it is found that ESC04a and ESC04b do a reasonable job while ESC04c and ESC04d do not (Halderson, 2008). In addition, the tensor interaction is too weak (wrong ordering of the ground-state doublet in ${ }_{\Lambda}^{16} \mathrm{O}$ ) and the $\Lambda N-\Sigma N$ coupling potentials have an unusual radial behavior. For the ESC08 models, the strength of the $\Lambda$ spin-dependent spin-orbit interaction has been reduced with respect to earlier models (Yamamoto, Motoba, and Rijken, 2010) as demanded by the data. However, the ordering of many doublets in the $p$-shell hypernuclei are inverted because the combination of attractive triplet-even and triplet-odd central interactions makes the triplet interaction stronger than the singlet $(\Delta<0)$. As noted in the section on $s$-shell hypernuclei, all of the models are missing something. In practice, empirical adjustments to the derived G-matrix interactions are made to fit the available data. Of course, these fits also cover for the missing three-body interactions, the effect of which is likely to be mostly on the absolute binding energies and on vector (SLS and ALS) interactions in the core nuclei (represented phenomenologically by $S_{N}$ ). 


\section{B. Weak decays of $\Lambda$ hypernuclei}

\section{Mesonic decays}

$\Lambda$ hypernuclei are unstable to weak decays of the $\Lambda$ hyperon. In free space, the $\Lambda$ weak-interaction lifetime $\tau_{\Lambda}=\hbar / \Gamma_{\Lambda}^{\text {free }}=2.632 \times 10^{-10} \mathrm{~s}$ is dominated $(99.7 \%)$ by nonleptonic, mesonic two-body decay (Olive et al., 2014):

$$
\begin{gathered}
\Lambda \rightarrow p+\pi^{-}+38 \mathrm{MeV} \quad(63.9 \pm 0.5) \% \\
\Lambda \rightarrow n+\pi^{0}+41 \mathrm{MeV} \quad(35.8 \pm 0.5) \%
\end{gathered}
$$

The ratio $\Gamma_{\Lambda \rightarrow p+\pi^{-}}^{\text {free }} / \Gamma_{\Lambda \rightarrow n+\pi^{0}}^{\text {free }}$ for these branches is close to 2 , in agreement with the $\Delta I=1 / 2$ rule (Boyle et al. 2013) which is also satisfied to a level of a few percent by all other known strangeness-changing nonleptonic weak decays, e.g. in kaon decays. In contrast, a purely $\Delta I=$ $3 / 2$ rule would give a branching ratio $1 / 2$. The effective $\Lambda \rightarrow N \pi$ weak decay Lagrangian is written as

$$
\mathcal{L}_{\Lambda N \pi}^{W}=-i G_{F} m_{\pi}^{2} \bar{\psi}_{N}\left(A+B \gamma_{5}\right) \boldsymbol{\tau} \cdot \boldsymbol{\phi}_{\pi} \psi_{\Lambda}
$$

where $G_{F} m_{\pi}^{2}=2.211 \times 10^{-7}$, and $A=1.06, B=-7.10$ are fixed by the measured free-space $\Lambda$ decay parameters. The isospin operator $\tau$ imposes the $\Delta I=1 / 2$ rule once the $\Lambda$ hyperon is assigned a fictitious isospin state $\left(I, I_{z}\right)=(1 / 2,-1 / 2)$. The nonrelativistic approximation to the free $\Lambda$ decay width yields

$$
\begin{aligned}
\Gamma_{\alpha}^{\text {free }}= & c_{\alpha}\left(G_{F} m_{\pi}^{2}\right)^{2} \int \frac{d^{3} \boldsymbol{q}}{(2 \pi)^{3} 2 \omega(\boldsymbol{q})} 2 \pi \delta\left(m_{\Lambda}-\omega(\boldsymbol{q})-E_{N}\right) \\
& \times\left(S^{2}+\frac{P^{2}}{m_{\pi}^{2}} \boldsymbol{q}^{2}\right)
\end{aligned}
$$

where $c_{\alpha}=1,2$ for $\alpha=\Lambda \rightarrow n \pi^{0}, \Lambda \rightarrow p \pi^{-}$, respectively, $S=A, P / m_{\pi}=B /\left(2 m_{N}\right)$, and $E_{N}$ and $\omega(\boldsymbol{q})$ are the total energies of the emitted nucleon and $\pi$ meson, respectively. This leads to the following expression for the total free-space decay width:

$$
\Gamma_{\Lambda}^{\text {free }}=\frac{3}{2 \pi}\left(G_{F} m_{\pi}^{2}\right)^{2} \frac{m_{N} q_{\text {c.m. }}}{m_{\Lambda}}\left(S^{2}+\frac{P^{2}}{m_{\pi}^{2}} q_{\text {c.m. }}^{2}\right),
$$

with $q_{\text {c.m. }} \approx 100 \mathrm{MeV} / \mathrm{c}$ for the pion momentum in the center-of-mass frame.

The empirical $\Delta I=1 / 2$ rule (Boyle et al. 2013 ) is not well understood. However here a key question is whether, and to what extent, it is satisfied by in medium $\Lambda$ weak decays. There has been no unambiguous experimetal test of the validity of this rule in hypernuclei. One reason is the difficulty to resolve two-body exclusive decay channels in the continuum, where a combination of several isospin values for the residual nucleus washes out the

\begin{tabular}{|c|c|c|c|}
\hline${ }_{\Lambda}^{A} \mathrm{Z}$ & $\Gamma_{\pi^{-}}$ & $\Gamma_{\pi^{0}}$ & Reference \\
\hline${ }_{\Lambda}^{4} \mathrm{He}$ & $0.289 \pm 0.039$ & $0.604 \pm 0.073$ & Parker et al. (2007) \\
\hline${ }_{\Lambda}^{5} \mathrm{He}$ & $0.340 \pm 0.016$ & $0.201 \pm 0.011$ & $\begin{array}{c}\text { Kameoka et al. (2005), } \\
\text { Okada et al. }(2005)\end{array}$ \\
\hline${ }_{\Lambda}^{12} \mathrm{C}$ & $0.123 \pm 0.015$ & $0.165 \pm 0.008$ & $\begin{array}{c}\text { Kameoka et al. (2005) } \\
\text { Okada et al. }(2005)\end{array}$ \\
\hline${ }_{\Lambda}^{28} \mathrm{Si}$ & $0.046 \pm 0.011$ & - & Sato et al. (2005) \\
\hline${ }_{\Lambda} \mathrm{Fe}$ & $\leq 0.015(90 \% \mathrm{CL})$ & - & Sato et al. (2005) \\
\hline
\end{tabular}
effect of the primary $\Delta I=1 / 2$ weak decay. For example, the total mesonic decay widths of ${ }_{\Lambda}^{4} \mathrm{He}$ given in Table $\mathrm{X}$ naively suggest that a $\Delta I=3 / 2$ rule holds.
TABLE X Measured total pionic decay widths of selected hypernuclei in units of $\Gamma_{\Lambda}^{\text {free }}$.

However, realizing the dominance of the two-body decay ${ }_{\Lambda}^{4} \mathrm{He} \rightarrow \pi^{0}+{ }^{4} \mathrm{He}$, and the impossibility of a $\pi^{-}+{ }^{4} \mathrm{He}$ two-body final state owing to charge conservation, the reversal of the $\pi^{-} / \pi^{0}$ ratio from close to 2 in the freespace decay to close to $1 / 2$ in ${ }_{\Lambda}^{4} \mathrm{He}$ decay only reflects the dominance of the ${ }^{4} \mathrm{He}$ ground-state branch. A similar trend is also seen in the $\pi^{-} / \pi^{0}$ ratio of ${ }_{\Lambda}^{12} \mathrm{C}$ total mesonic decay widths listed in the table. On the other hand, the $\pi^{-} / \pi^{0}$ ratio for ${ }_{\Lambda}^{5} \mathrm{He}$ is close to the free-space ratio, reflecting the difficulty to divert sufficient kinetic energy to break up the ${ }^{4} \mathrm{He}$ core in the quasi-free decays ${ }_{\Lambda}^{5} \mathrm{He} \rightarrow{ }^{4} \mathrm{He}+N+\pi$. The systematics of the $\pi^{-} / \pi^{0}$ ratio, owing to the nuclear structure of $p$-shell $\Lambda$ hypernuclei, was discussed by Motoba et al. (1988).

Another reason for the difficulty of testing the $\Delta I=$ $1 / 2$ rule in mesonic decays of hypernuclei is the rapid decrease of the pionic decay width $\Gamma_{\pi}=\Gamma_{\pi^{-}}+\Gamma_{\pi^{0}}$ as a function of hypernuclear mass number $A$. This is shown in Table $\mathrm{X}$ where some of the latest determinations of $\pi^{-}$ decay widths in hypernuclei for $A \geq 11$ are listed (Sato et al. 2005). The pionic decay widths fall off from about $0.9 \Gamma_{\Lambda}^{\text {free }}$ in ${ }_{\Lambda}^{4}$ He to few percent in ${ }_{\Lambda} \mathrm{Fe}$. This had been anticipated from the low momentum $q \approx 100 \mathrm{MeV} / \mathrm{c}$, $q<p_{F}$, of the nucleon recoil in the pionic decay and was indeed confirmed quantitatively by detailed calculations of mesonic decay of $\Lambda$ hypernuclei. Equation (32) for the free-space decay width is replaced in hypernuclei by

$$
\begin{aligned}
\Gamma_{\alpha}= & c_{\alpha}\left(G_{F} m_{\pi}^{2}\right)^{2} \sum_{f} \int \frac{d^{3} \boldsymbol{q}}{(2 \pi)^{3} 2 \omega(\boldsymbol{q})} 2 \pi \delta\left(E_{\Lambda}-\omega(\boldsymbol{q})-E_{N}^{f}\right) \\
& \times\left(S^{2}\left|\int d^{3} \boldsymbol{r} \phi_{\Lambda}(\boldsymbol{r}) \phi_{\pi}(\boldsymbol{r} ; \boldsymbol{q}) \phi_{f}^{*}(\boldsymbol{r})\right|^{2}\right. \\
& \left.+\frac{P^{2}}{m_{\pi}^{2}}\left|\int d^{3} \boldsymbol{r} \phi_{\Lambda}(\boldsymbol{r}) \boldsymbol{\nabla} \phi_{\pi}(\boldsymbol{r} ; \boldsymbol{q}) \phi_{f}^{*}(\boldsymbol{r})\right|^{2}\right),
\end{aligned}
$$

where the sum extends over the unoccupied nucleon states $f$, and the pion wavefunction $\phi_{\pi}(\boldsymbol{r} ; \boldsymbol{q})$ is a solution of the Klein Gordon equation in the presence of a pion-nuclear optical potential $V_{\text {opt }}$ :

$$
\left\{\nabla^{2}-m_{\pi}^{2}-2 \omega(\boldsymbol{q}) V_{\mathrm{opt}}(\boldsymbol{r})+\left[\omega-V_{c}(\boldsymbol{r})\right]^{2}\right\} \phi_{\pi}(\boldsymbol{r} ; \boldsymbol{q})=0 .
$$

The free-space Eq. (32) is recovered from Eq. (34) by extending the sum over occupied nucleon states as well, 
neglecting the pion-nuclear final-state interaction, i.e., $\phi_{\pi}^{\text {free }}(\boldsymbol{r} ; \boldsymbol{q})=\exp \left(i \boldsymbol{q}_{\mathrm{c} . \mathrm{m} .} \cdot \boldsymbol{r}\right)$, and using closure. The reduction of the mesonic decay width in hypernuclei by several orders of magnitudes as $A$ increases is due to limiting the sum to unoccupied nucleon states. In realistic calculations, however, the final-state nuclear interaction of the emitted pion plays a significant role, providing enhancement of the decay rate in heavy hypernuclei by one to two orders of magnitude over what a plane-wave impulse approximation calculation (using $\phi_{\pi}^{\text {free }}(\boldsymbol{r} ; \boldsymbol{q})$ ) would give (Oset and Salcedo, 1985; Itonaga, Motoba, and Bandō, 1988; Nieves and Oset, 1993; Motoba and Itonaga, 1994).

A weak $\pi^{+}$decay branch with width of order $0.02 \Gamma_{\Lambda}^{\text {free }}$ was observed in the decay of ${ }_{\Lambda}^{4} \mathrm{He}$ in emulsion studies (Bohm et al. 1969$)$ and in helium bubble chambers (Fetkovich et al., 1972). Weaker evidence exists for $\pi^{+}$ decay of ${ }_{\Lambda}^{7}$ Be observed in emulsion. The rare $\pi^{+}$branch was initially studied theoretically by Dalitz and von Hippel (Dalitz and von Hippel, 1964b; von Hippel, 1964) who observed that it required an intermediate stronginteraction step to occur through, e.g. (i) $\Lambda \rightarrow n+\pi^{0}$ followed by $\left(\pi^{0}, \pi^{+}\right)$charge exchange in the final state, or (ii) $\Lambda p \rightarrow \Sigma^{+} n$, in order to generate a virtual $\Sigma^{+}$component in the initial $\Lambda$ hypernuclear wavefunction followed by $\Sigma^{+} \rightarrow n+\pi^{+}$. The pion charge-exchange mechanism was recalculated by Cieplý and Gal (1997) where its rate was found larger than in the original calculation (Dalitz and von Hippel, 1964b), but still short by about a factor of two with respect to the observed rate. Gibson and Timmermans (1998) argued that relatively large $\Sigma^{+}$admixtures were unique to ${ }_{\Lambda}^{4} \mathrm{He}$ and could explain the large $\pi^{+}$rates observed.

The study of exclusive two-body pionic weak decays of light hypernuclei has yielded valuable information on the ground-state spins of several species, as summarized in Table XI These pionic weak decays show selectivity to the spin of the hypernuclear ground state owing to the dominance (88\%) of the $s$-wave, parity-violating $\Lambda \rightarrow N \pi$ amplitude [ $A$ term in Eq. [31]]. This is demonstrated in Fig. 19, taken from a recent FINUDA work Agnello et al. 2009), showing a $\pi^{-}$weak-decay spectrum for ${ }_{\Lambda}^{15} \mathrm{~N}$, with a preference for a g.s. spin $3 / 2^{+}$for ${ }_{\Lambda}^{15} \mathrm{~N}($ Gal, 2009). In terms of nuclear-core spin $J_{c}$ values the derived hypernuclear spins $J$ satisfy $J=J_{c}-\frac{1}{2}$ in the $s$ shell and $p_{\frac{3}{2}}$ subshell, and $J=J_{c}+\frac{1}{2}$ for ${ }_{\Lambda}^{15} \mathrm{~N}$ in the $p_{\frac{1}{2}}$ subshell, all consistent with the $\Lambda N$ spin-singlet interaction being stronger than the spin-triplet interaction.

\section{Nonmesonic decays}

$\Lambda$ hypernuclear total decay widths $\Gamma_{\Lambda}$ are known to remain close to the free- $\Lambda$ decay width $\Gamma_{\Lambda}^{\text {free }}$, in spite of the rapid decrease as a function of $A$ of the $\Lambda \rightarrow N \pi$ mesonic weak decay (MWD) widths $\Gamma_{\pi}$, as demonstrated in Table $\mathrm{X} \mathrm{A}$ new mode of nonmesonic weak decay

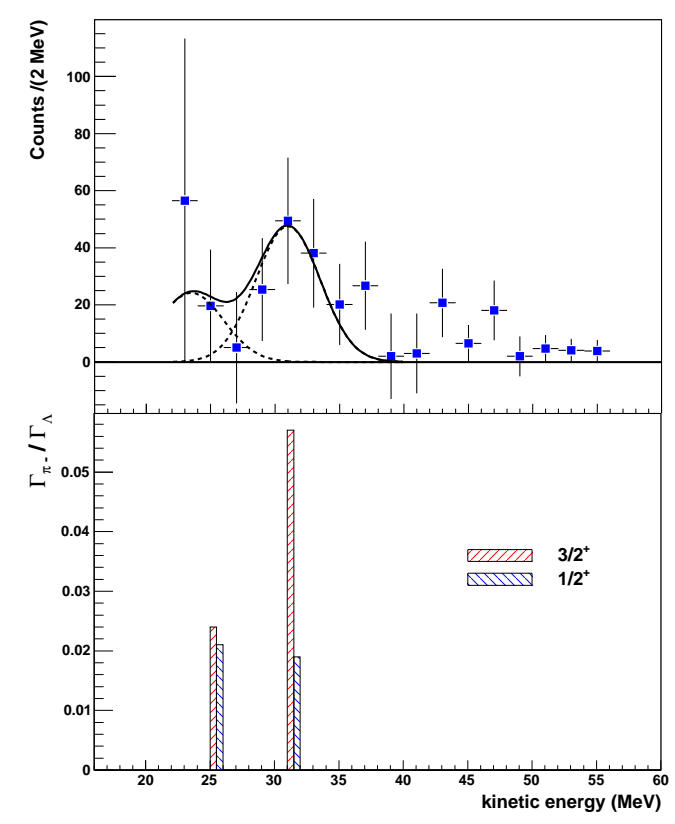

FIG. 19 Mesonic weak-decay spectrum of ${ }_{\Lambda}^{15} \mathrm{~N} \rightarrow \pi^{-}+{ }^{15} \mathrm{O}$ (upper panel) observed at DA $\Phi$ NE by the FINUDA Collaboration, compared to calculations (lower panel) for the two possible spin values of the decaying $\Lambda$ hypernucleus (Gal, 2009) which show preference for a ${ }_{\Lambda}^{15} \mathrm{~N}$ g.s. spin $3 / 2^{+}$. Adapted from Agnello et al., 2009.

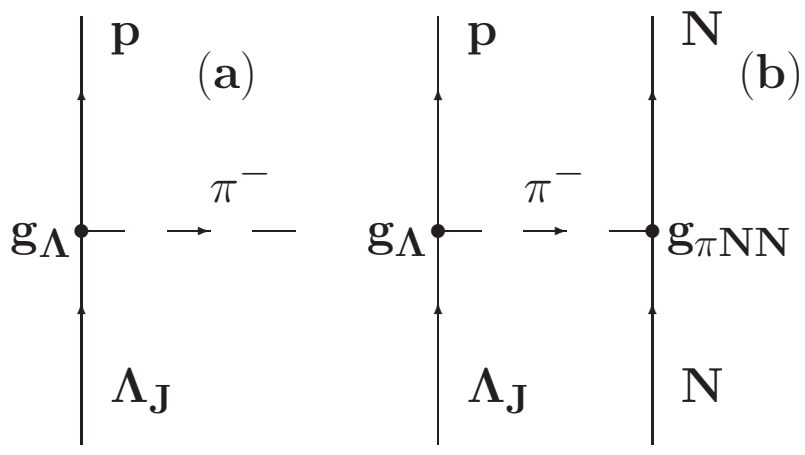

FIG. 20 Graph (a) is for mesonic $\Lambda_{J} \rightarrow p \pi^{-}$decay, where $\Lambda_{J}$ denotes a $\Lambda$ hyperon of total spin $J$. Graph (b) depicts nonmesonic de-excitation for a $\Lambda_{J}$ hyperon in nuclear matter. Adapted from Dalitz, 2005.

(NMWD), predicted by Cheston and Primakoff (1953), emerges upon increasing $A$ through the absorption of a weak-decay, virtual pion on one or more nucleons, as illustrated in Fig. 20, Other weak-decay virtual mesons may also mediate these NMWD modes. Historically, Karplus and Ruderman (1956) used the observed rates of the nonmesonic weak decay of $\Lambda$ hypernuclei to argue that the spin of the $\Lambda$ hyperon was consistent with $J_{\Lambda}=1 / 2$, and that there was no need to ascribe the relatively long lifetimes of strangeness weak decays to an exceptionally 
TABLE XI Hypernuclear spin assignments provided by pionic weak-decay studies.

\begin{tabular}{|c|c|c|c|c|}
\hline${ }_{\Lambda}^{A} \mathrm{Z}$ & $J^{\pi}$ & Decay branch & Theory & Experiment \\
\hline${ }_{\Lambda}^{3} \mathrm{H}$ & $\frac{1}{2}^{+}$ & $\pi^{-}+{ }^{3} \mathrm{He}$ & Dalitz and Liu (1959) & $\begin{array}{l}\text { Ammar, Dunn, and Holland (1962), } \\
\text { Block et al. (1964), and Bertrand } \\
\text { et al. (1970) }\end{array}$ \\
\hline${ }_{\Lambda}^{4} \mathrm{He}$ & $0^{+}$ & $\pi^{0}+$ all & Dalitz and Liu (1959) & $\begin{array}{l}\text { Block et al. (1964) and Fetkovich } \\
\text { et al. (1972) }\end{array}$ \\
\hline${ }_{\Lambda}^{8} \mathrm{Li}$ & $1^{-}$ & $\pi^{-}+{ }^{4} \mathrm{He}+{ }^{4} \mathrm{He}$ & Dalitz (1963a) & Davis, Levi Setti, and Raymund (1963) \\
\hline${ }_{\Lambda}^{11} \mathrm{~B}$ & $\frac{5}{2}^{+}$ & $\pi^{-}+{ }^{11} \mathrm{C}^{*}(6.48 \mathrm{MeV})$ & Ziemińska (1975) & Jurič et al. (1973) \\
\hline${ }_{\Lambda}^{12} \mathrm{~B}$ & $1^{-}$ & $\pi^{-}+{ }^{4} \mathrm{He}+{ }^{4} \mathrm{He}+{ }^{4} \mathrm{He}$ & $\begin{array}{l}\text { Ziemińska and Dalitz (1975) } \\
\text { and Kielczewska, Ziemińska, } \\
\text { and Dalitz (1975) }\end{array}$ & Kielczewska et al. (1975) \\
\hline
\end{tabular}

large value of $J_{\Lambda}$.

The dominant NMWD modes are believed to involve one nucleon in the initial state:

$$
\begin{gathered}
\Lambda+p \rightarrow n+p+176 \mathrm{MeV} \quad\left(\Gamma_{p}\right), \\
\Lambda+n \rightarrow n+n+176 \mathrm{MeV} \quad\left(\Gamma_{n}\right),
\end{gathered}
$$

having a summed width $\Gamma_{1}=\Gamma_{p}+\Gamma_{n}$. Two-nucleon $(2 N)$ modes are also possible (Alberico et al., 1991),

$$
\Lambda+N+N \rightarrow n+N+N+176 \mathrm{MeV}\left(\Gamma_{2}\right) .
$$

A conservative estimate given by Alberico et al. (1991) for these decays is $\Gamma_{2} / \Gamma_{1} \sim 0.2$. The total hypernuclear weak-decay width, $\Gamma_{\Lambda}=\Gamma_{\pi}+\Gamma_{\mathrm{nm}}$, is a sum of the MWD width $\Gamma_{\pi}$ and the NMWD width, denoted by $\Gamma_{\mathrm{nm}}=\Gamma_{1}+\Gamma_{2}+\cdots$. The dots stand for more involved multinucleon decay modes. Very little is known about multinucleon decay modes beyond the two-nucleon mode as most experimental and theoretical studies of $\Lambda$ hypernuclear weak decay have focused on the one-nucleon modes, Eqs. (36) and (37). The branching ratio of the $2 N$ NMWD contribution to the total ${ }_{\Lambda}^{12} \mathrm{C}$ NMWD width has been determined in KEK (Kim et al., 2009$)$ and in DA $\Phi$ NE (Agnello et al., 2011c) experiments, with values given by

$$
\frac{\Gamma_{2}}{\Gamma_{\mathrm{nm}}}=0.29 \pm 0.13, \quad 0.21 \pm 0.08,
$$

respectively. The latter value was derived from analysis of several NMWD spectra, assuming that this branching ratio is constant in the $p$ shell. The $2 N$ NMWD mode was observed recently through a complete kinematical reconstruction of a three-nucleon final state in two ${ }_{\Lambda}^{7} \mathrm{Li} \rightarrow$ ${ }^{4} \mathrm{He}+n+n+p$ decay events at DA $\Phi$ NE Agnello et al. 2012b), as demonstrated earlier in Fig. 3 .
NMWD dominates the $\Lambda$-hypernuclear decay in all but the lightest hypernuclei. This is demonstrated in Fig. 21 where $\Gamma_{p}$, the largest contributor to NMWD, and $\Gamma_{\pi^{-}}$, the largest contributor to MWD, are shown as a function of $A$ along the $p$ shell as determined by FINUDA and in comparison to various calculations. It is seen clearly that $\Gamma_{p}$ rises roughly by a factor of 2 , whereas $\Gamma_{\pi^{-}}$decreases roughly by a factor of 3 from ${ }_{\Lambda}^{5} \mathrm{He}$ to ${ }_{\Lambda}^{15} \mathrm{~N}$, with the ratio $\Gamma_{p} / \Gamma_{\pi^{-}}$reaching a value somewhat larger than 4 at the end of the $p$ shell. NMWD is the only practical way to study the four-fermion, weak-decay interaction. The relatively large momentum transfer, $\approx 420 \mathrm{MeV} / \mathrm{c}$ in free space, could mean that sub-nucleon degrees of freedom are important, but at the present level of experimental data there seems no advantage to invoke explicitly sub-nucleon models. The status of models that consider direct quark (DQ) processes, in addition to meson exchanges, is summarized by Sasaki, Izaki, and Oka (2005). DQ models offer a natural theoretical framework for departing from the $\Delta I=1 / 2$ rule. However, there is no compelling evidence so far that this rule is not satisfied in $\Lambda$ hypernuclear NMWD. The models reviewed here are hadronic models that are built upon meson exchanges for which the $\Delta I=1 / 2$ rule is assumed to hold. A common approximation is that NMWD occurs dominantly from $s$-wave $\Lambda N$ states owing to the short range nature of these decays. The possible $\Lambda+N \rightarrow N+N$ transitions are listed in Table XII as taken from Block and Dalitz (1963), together with the spin dependence of the corresponding matrix elements. Thus, for capture from ${ }^{1} S_{0}$ states, parity nonconservation in the weak interactions allows both the parity-conserving (PC) ${ }^{1} S_{0} \rightarrow{ }^{1} S_{0}$ as well as the parity-violating $(\mathrm{PV})^{1} S_{0} \rightarrow{ }^{3} P_{0}$ transitions. Of the six amplitudes listed, those with $a, c$, and $d$ are $\mathrm{PC}$ and those with $b, e$, and $f$ are $\mathrm{PV}$; those with $c, d$, and $e$, leading to $I=0 N N$ states, are unique to 

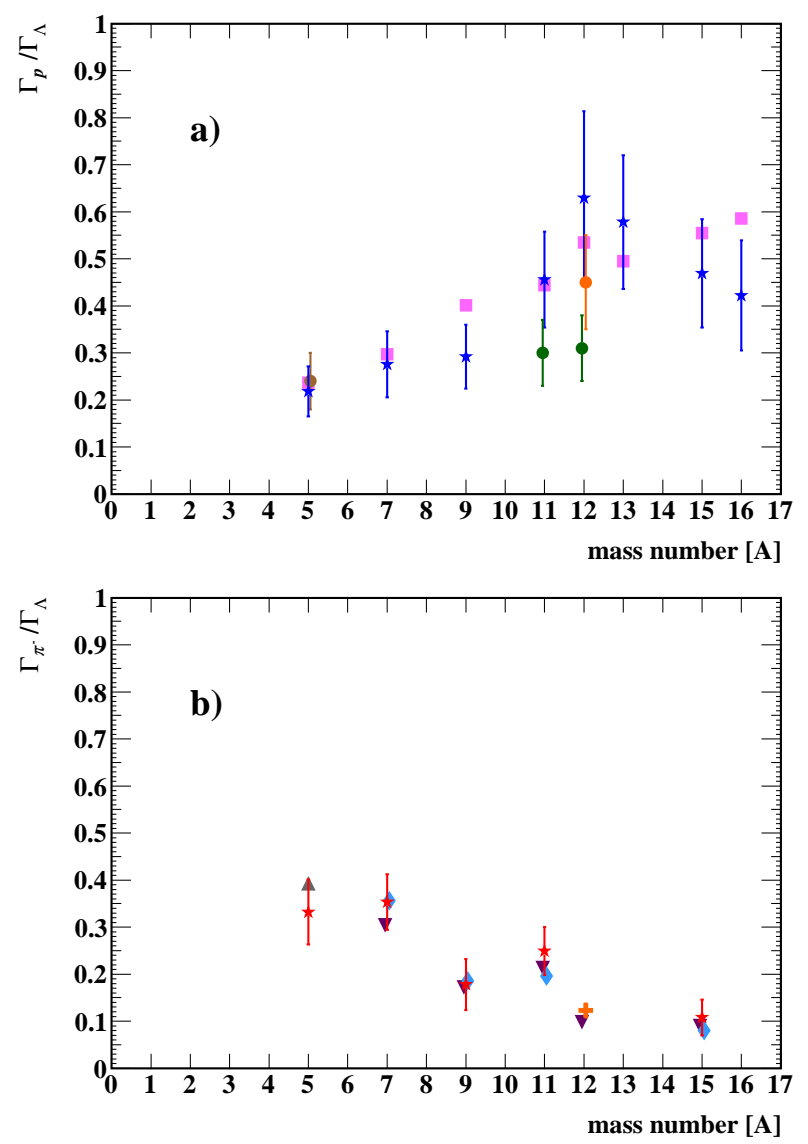

FIG. $21 \Gamma_{p}$ (blue stars, upper panel) and $\Gamma_{\pi^{-}}$(red stars, lower panel), in units of the free $\Lambda$ decay width, as a function of $A$ from measurements and analysis reported by the FINUDA Collaboration (Agnello et al., 2009, 2014). Other experimental results and theoretical calculations are also marked, see caption to the original Fig. 3 in Agnello et al., 2014.

$\Lambda p \rightarrow n p$ whereas for the $a, b$, and $f$ amplitudes, which lead to $I=1 N N$ states, both $n n$ and $n p$ final states are possible with $a_{n}=\sqrt{2} a_{p}, b_{n}=\sqrt{2} b_{p}, f_{n}=\sqrt{2} f_{p}$ satisfying the $\Delta I=1 / 2$ rule.

TABLE XII $\Lambda+N \rightarrow N+N$ amplitudes Block and Dalitz, 1963). The Pauli spin operator, $\sigma_{\Lambda}$, acts on the initial $\Lambda$ particle and the final neutron. The final neutron momentum is $\boldsymbol{q}$, and $\boldsymbol{Q} \equiv \boldsymbol{q} / m_{N}$.

\begin{tabular}{cccc}
\hline \hline Transition & Operator & $I_{N N}$ & Rate \\
${ }^{1} S_{0} \rightarrow{ }^{1} S_{0}$ & $\frac{a}{4}\left(1-\boldsymbol{\sigma}_{\Lambda} \cdot \boldsymbol{\sigma}_{N}\right)$ & 1 & $|a|^{2}$ \\
${ }^{1} S_{0} \rightarrow{ }^{3} P_{0}$ & $\frac{b}{8}\left(\boldsymbol{\sigma}_{\Lambda}-\boldsymbol{\sigma}_{N}\right) \cdot \boldsymbol{Q}\left(1-\boldsymbol{\sigma}_{\Lambda} \cdot \boldsymbol{\sigma}_{N}\right)$ & 1 & $|b|^{2} Q^{2}$ \\
${ }^{3} S_{1} \rightarrow{ }^{3} S_{1}$ & $\frac{c}{4}\left(3+\boldsymbol{\sigma}_{\Lambda} \cdot \boldsymbol{\sigma}_{N}\right)$ & 0 & $|c|^{2}$ \\
${ }^{3} S_{1} \rightarrow{ }^{3} D_{1}$ & $\frac{3 d}{\sqrt{2}}\left(\boldsymbol{\sigma}_{\Lambda} \cdot \boldsymbol{Q} \boldsymbol{\sigma}_{N} \cdot \boldsymbol{Q}-\frac{1}{3} \boldsymbol{\sigma}_{\Lambda} \cdot \boldsymbol{\sigma}_{N} Q^{2}\right)$ & 0 & $|d|^{2} Q^{4}$ \\
${ }^{3} S_{1} \rightarrow{ }^{1} P_{1}$ & $\frac{e \sqrt{3}}{8}\left(\boldsymbol{\sigma}_{\Lambda}-\boldsymbol{\sigma}_{N}\right) \cdot \boldsymbol{Q}\left(3+\boldsymbol{\sigma}_{\Lambda} \cdot \boldsymbol{\sigma}_{N}\right)$ & 0 & $|e|^{2} Q^{2}$ \\
${ }^{3} S_{1} \rightarrow{ }^{3} P_{1}$ & $\frac{f \sqrt{6}}{4}\left(\boldsymbol{\sigma}_{\Lambda}+\boldsymbol{\sigma}_{N}\right) \cdot \mathbf{Q}$ & 1 & $|f|^{2} Q^{2}$ \\
\hline \hline
\end{tabular}

It is instructive to show the structure of the one-pionexchange (OPE) transition potential generated by the
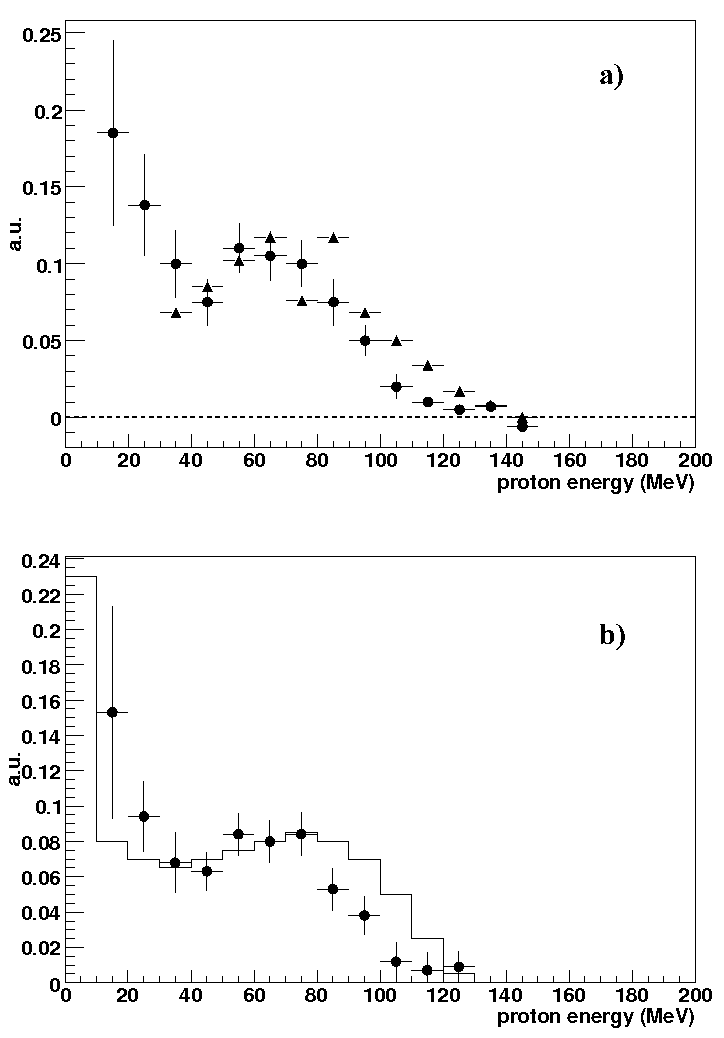

FIG. 22 Upper panel: proton-energy spectrum from ${ }_{\Lambda}^{5}$ He nonmesonic weak decay measured by FINUDA (circles) and at KEK (triangles). The two spectra were normalized beyond 35 $\mathrm{MeV}$ (threshold of the KEK spectrum). Lower panel: comparison between the FINUDA proton-energy spectrum (circles) from the upper panel and the INC calculation (histogram) of (Garbarino, Parreño, and Ramos 2004). The two spectra were normalized beyond $15 \mathrm{MeV}$ (threshold of the FINUDA spectrum). Adapted from Agnello et al., 2008.

diagram of Fig. 20p. To this end, the weak-interaction Lagrangian Eq. (31) is augmented by a strong-interaction component

$$
\mathcal{L}_{N N \pi}^{S}=-i g_{N N \pi} \bar{\psi}_{N} \gamma_{5} \boldsymbol{\tau} \cdot \boldsymbol{\phi}_{\pi} \psi_{N},
$$

where $g_{N N \pi}=13.2$ is the strong-interaction coupling constant. Including the pion propagator between the two vertices given by Eqs. (31) and 40) and applying a nonrelativistic reduction, one obtains the OPE momentumspace transition potential

$$
\begin{aligned}
V_{\mathrm{OPE}}(\boldsymbol{q})= & -G_{F} m_{\pi}^{2} \frac{g_{N N \pi}}{2 m_{N}}\left(A+\frac{B}{2 m_{\mathrm{av}}} \boldsymbol{\sigma}_{\Lambda} \cdot \boldsymbol{q}\right) \\
& \times \frac{\boldsymbol{\sigma}_{N} \cdot \boldsymbol{q}}{\boldsymbol{q}^{2}+m_{\pi}^{2}} \boldsymbol{\tau}_{\Lambda} \cdot \boldsymbol{\tau}_{N},
\end{aligned}
$$

where $m_{\mathrm{av}}=\left(m_{N}+m_{\Lambda}\right) / 2$. The OPE potential, owing to the sizable momentum transfer involved, is dominated by the tensor component, amplitude $d$ of Table XII. For this amplitude the final $N N$ state has isospin $I=0$, 
TABLE XIII Measured and calculated NMWD widths and related entities for selected hypernuclei in units of $\Gamma_{\Lambda}^{\text {free }}$.

\begin{tabular}{|c|c|c|c|}
\hline Entity & Method & ${ }_{\Lambda}^{5} \mathrm{He}$ & ${ }_{\Lambda}^{12} \mathrm{C}$ \\
\hline$\Gamma_{n} / \Gamma_{p}$ & 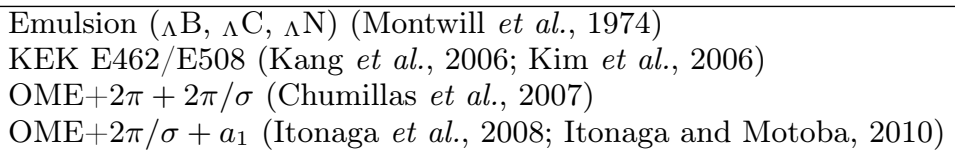 & $\begin{aligned} 0.45 \pm & 0.11 \pm 0.03 \\
& 0.415 \\
& 0.508\end{aligned}$ & $\begin{array}{l}0.59 \pm 0.15 \\
0.51 \pm 0.13 \pm 0.05 \\
0.366 \\
0.418\end{array}$ \\
\hline$\Gamma_{n m}$ & $\begin{array}{l}\text { KEK E462/E508 (Okada et al. } 2005) \\
\mathrm{OME}+2 \pi+2 \pi / \sigma \text { (Chumillas et al. } 2007) \\
\mathrm{OME}+2 \pi / \sigma+a_{1}(\text { Itonaga et al., 2008; Itonaga and Motoba, 2010) }\end{array}$ & $\begin{array}{l}0.406 \pm 0.020 \\
0.388 \\
0.358\end{array}$ & $\begin{array}{l}0.953 \pm 0.032 \\
0.722 \\
0.758\end{array}$ \\
\hline$\Gamma_{\Lambda}$ & KEK E462/E508 (Kameoka et al., 2005$)$ & $0.947 \pm 0.038$ & $1.242 \pm 0.042$ \\
\hline$a_{\Lambda}$ & $\begin{array}{l}\text { KEK E462/E508 (Maruta et al. }, 2007) \\
\text { OME (Chumillas et al. } 2007,2008) \\
\text { With final-state interactions } \\
\text { OME }+2 \pi+2 \pi / \sigma \text { (Chumillas } \text { et al. } 2007,2008) \\
\text { With final-state interactions } \\
\text { OME }+2 \pi / \sigma+a_{1} \text { (Itonaga et al., 2008; Itonaga and Motoba, 2010) }\end{array}$ & $\begin{array}{c}0.07 \pm 0.08+0.08 \\
\quad-0.590 \\
-0.401 \\
+0.041 \\
+0.028 \\
+0.083\end{array}$ & $\begin{aligned}-0.16 & \pm 0.28+0.18 \\
& -0.698 \\
& -0.340 \\
& -0.207 \\
& -0.126 \\
& +0.044\end{aligned}$ \\
\hline
\end{tabular}

which is allowed for $n p$ but forbidden for $n n$. Thus, the full OPE transition potential calculations produce a small value for $\Gamma_{n} / \Gamma_{p} \leq 0.1$. This is considerably smaller than the range of values, $\Gamma_{n} / \Gamma_{p} \sim 0.5$, deduced from old nuclear emulsion work (Montwill et al. 1974 ) and from the most recent KEK experiments (Kang et al., 2006; Kim et al. 2006), indicating that OPE is insufficient to describe quantitatively NMWD.

In a semiclassical description of the hypernuclear $\Lambda+$ $N \rightarrow n+N$ decay, the energy of each one of the two outgoing nucleons should peak at roughly $80 \mathrm{MeV}$ which, assuming equal sharing of the released energy, is about half of the energy available in the decay. A proton-energy spectrum, taken by the FINUDA Collaboration Agnello et al. 2008) from nonmesonic weak decay of ${ }_{\Lambda}^{5} \mathrm{He}$ produced on thin Li targets, is shown in the upper part of Fig. 22 (circles) in comparison with a proton spectrum taken at KEK (Okada et al., 2004) (triangles). The two spectra were normalized above $35 \mathrm{MeV}$ which is the KEK proton-energy threshold. A peak around $60-90 \mathrm{MeV}$ is clearly observed, with a low-energy rise due to final state interactions (FSI), and perhaps also due to multinucleon induced weak decay. The FINUDA proton spectrum is compared in the lower part of Fig. 22 with the theoretical spectrum calculated by Garbarino, Parreño, and Ramos (2004) using an intranuclear cascade (INC) code. The two spectra were normalized above $15 \mathrm{MeV}$ which is the FINUDA proton-energy threshold. The agreement between experiment and theory is only qualitative. A more refined methodology to extract NMWD information from the FINUDA measured proton spectra has been presented recently by Agnello et al. (2014). Neutronenergy spectra were reported by the KEK-PS Experiments 462/508 (Okada et al., 2004), with a shape similar to that of the proton spectrum shown here, and with a similar rise at low energies. We note that the proton and neutron yields, $N_{p}$ and $N_{n}$ respectively, when properly normalized are related to the one-nucleon widths by

$$
N_{p}=\Gamma_{p}, \quad N_{n}=\Gamma_{p}+2 \Gamma_{n}
$$

These expressions disregard FSI and multinucleon stimulated decays.

In the KEK experiments, the number of $n p$ pairs, $N_{n p}$, and $n n$ pairs, $N_{n n}$, corresponding to back-to-back finalstate kinematics were identified and determined. Assuming that FSI has a negligible effect on the ratio $N_{n n} / N_{n p}$, the ratio $\Gamma_{n} / \Gamma_{p}$ was approximated by $N_{n n} / N_{n p}$ and the reported values for ${ }_{\Lambda}^{5} \mathrm{He}$ and ${ }_{\Lambda}^{12} \mathrm{C}$ are listed in Table XIII. For ${ }_{\Lambda}^{12} \mathrm{C}$ the KEK result agrees within error bars with the old emulsion value. A recent reevaluation of the KEK spectra by Bauer et al. (2010), accounting also for FSI, leads to a value of $\Gamma_{n} / \Gamma_{p}=0.66 \pm 0.24$, in agreement with the emulsion and KEK values cited in the table. Previous determinations of $\Gamma_{n} / \Gamma_{p}$ from single-nucleon spectra gave considerably higher values, often in the range $1-2$, but are understood at present to have been subject to strong and unaccounted for FSI effects. This caveat refers, in principle, also to the value cited in the table from emulsion work, which was obtained by matching the experimentally observed fast $\left(T_{p}>30 \mathrm{MeV}\right)$ proton spectrum with appropriately weighted spectra from MonteCarlo INC simulations of both proton and neutron FSI processes (recall that neutrons are not observed directly in emulsion). However, the emulsion estimate of $\Gamma_{n} / \Gamma_{p}$ appears to agree with the result of the more refined KEK analysis. Finally, two recent calculations using onemeson exchanges (OME) beyond OPE are listed in the table (Chumillas et al., 2007; Itonaga et al., 2008). These calculations reproduce satisfactorily the $\Gamma_{n} / \Gamma_{p}$ values deduced from the experiments listed in the table. They include also two-pion-exchange processes, with or without coupling the $\Lambda N$ system to $\Sigma N$, plus the two-pion $\left(J^{\pi}=0^{+}, I=0\right)$ resonance known as $\sigma$ and the axial vector meson $a_{1}$ considered as a $\rho-\pi$ resonance. The ad- 
dition of $\sigma$ and $a_{1}$ exchanges does not effectively change the $\Gamma_{n} / \Gamma_{p}$ ratio, but proves to be significant in the calculation of the $\Lambda$ asymmetry parameter as discussed below. Earlier calculations by Jido, Oset, and Palomar (2001), using a chiral-interaction EFT approach, gave a very similar result, $\Gamma_{n} / \Gamma_{p}=0.53$ in ${ }_{\Lambda}^{12} \mathrm{C}$.

Shown also in Table XIII are experimentally deduced, as well as calculated values of the total NMWD width $\Gamma_{n m}$ for ${ }_{\Lambda}^{5} \mathrm{He}$ and ${ }_{\Lambda}^{12} \mathrm{C}$. The deduced NMWD width more than doubles between ${ }_{\Lambda}^{5} \mathrm{He}$ and ${ }_{\Lambda}^{12} \mathrm{C}$ and is already close to saturation for $A=12$. Both calculations reproduce well the deduced NMWD width in ${ }_{\Lambda}^{5} \mathrm{He}$, but fall short of it in ${ }_{\Lambda}^{12} \mathrm{C}$, perhaps due to the increased role of the $2 N$ branch which was not included in the calculation. However, earlier calculations using the same exchanges, but with somewhat different couplings and with different prescriptions for the short-range behavior of the OME exchanges, were able to produce values $\Gamma_{\mathrm{nm}}\left({ }_{\Lambda}^{12} \mathrm{C}\right) \sim(1.0-1.2) \Gamma_{\Lambda}^{\text {free }}$ (Itonaga, Ueda, and Motoba, 2002; Barbero et al., 2003). On the other hand, a more recent calculation by Bauer and Garbarino (2010), considering g.s. short-range correlations and including consistently a $2 N$ branch, $\Gamma_{2} / \Gamma_{n m}=0.26$, obtained a value $\Gamma_{\mathrm{nm}}=0.98 \Gamma_{\Lambda}^{\text {free }}$, in very good agreement with the KEK deduced NMWD width. The saturation of the NMWD width for large values of $A$ is demonstrated in Table VI where total hypernuclear decay lifetimes measured to better than $10 \%$ accuracy are displayed. Recall from Table $\mathrm{X}$ that for $A=56$ the mesonic decay width is no more than few percent of the nonmesonic width, hence the total width (lifetime) agrees to this accuracy with the nonmesonic width (lifetime).

In the $\Lambda+N \rightarrow n+N$ two-body reactions, each of the final-state nucleons receives a momentum (energy) of order $400 \mathrm{MeV} / \mathrm{c}(80 \mathrm{MeV})$, which is well above the Fermi momentum (energy). This large value of momentum transfer justifies the use of semiclassical estimates for inclusive observables, such as the total nonmesonic decay rate of $\Lambda$ hypernuclei. Denoting a properly spin-isospin averaged nonmesonic decay width on a bound nucleon in nuclear matter by $\bar{\Gamma}_{\Lambda}$, the total hypernuclear rate is given in the local density approximation by

$$
\frac{\bar{\Gamma}_{\Lambda}}{\rho_{0}} \int \rho_{\Lambda}(r) \rho_{N}(r) \mathrm{d}^{3} r,
$$

where $\rho_{\Lambda}(r)$ and $\rho_{N}(r)$ are the $\Lambda$ and the nucleon densities, normalized to 1 and to $A$, respectively, $\rho_{0}$ denotes nuclear-matter density, and zero range was implicitly assumed for the $\Lambda+N \rightarrow n+N$ amplitudes. Approximating the nucleon density $\rho_{N}(r)$ by $\rho_{0}$ for values of $r$ over which the $0 s_{\Lambda}$ density $\rho_{\Lambda}(r)$ is localized, Eq. 43 reduces to $\bar{\Gamma}_{\Lambda}$, independently of $A$. For nuclei with $N \neq Z$, the limiting value $\bar{\Gamma}_{\Lambda}$ is replaced by

$$
\bar{\Gamma}_{\Lambda}^{0}+\bar{\Gamma}_{\Lambda}^{1} \frac{N-Z}{A}=\Gamma_{n} \frac{N}{A}+\Gamma_{p} \frac{Z}{A},
$$

where $\bar{\Gamma}_{\Lambda}^{0}=\left(\Gamma_{n}+\Gamma_{p}\right) / 2$ and $\bar{\Gamma}_{\Lambda}^{1}=\left(\Gamma_{n}-\Gamma_{p}\right) / 2$. Equation (44) provides the leading term in a systematic expansion in powers of the neutron excess parameter $(N-Z) / A$. Finally, accepting that mesonic partial decay widths become negligible in medium- and heavierweight hypernuclei and the total decay widths are essentially given by the nonmesonic decay widths, the total nonmesonic decay rate is expected to saturate in heavy hypernuclei, as was demonstrated in Table VI.

The last item in Table XIII concerns the $\Lambda$ intrinsic asymmetry parameter $a_{\Lambda}$ in the nonmesonic weak decay Eq. 36 of polarized $\Lambda$ hypernuclei. The angular distribution of the decay protons is given by

$$
W(\theta)=W_{0}\left(1+a_{\Lambda} \mathcal{P}_{\Lambda} \cos \theta\right)
$$

where $\mathcal{P}_{\Lambda}$ is the polarization of the $\Lambda$ spin in the decaying hypernucleus [as produced, e.g., in $\left(\pi^{+}, K^{+}\right)$reactions] and $\theta$ is the emission angle of the protons with respect to the polarization axis. The asymmetry arises from the interference between PC and the PV weak-decay amplitudes. The values of $a_{\Lambda}$ deduced from experiment and listed in the table are close to zero, in strong disagreement with OME calculations; see, e.g., Parreño, Ramos, and Bennhold (1997) and Parreño and Ramos (2001). A more recent representative example for such calculations is shown in Table XIII. This long-standing problem was recently resolved with the introduction of a scalarisoscalar $\left(0^{+}, 0\right)$ exchange which reduces the size of the negative and large asymmetry parameter produced in the OME calculations (Sasaki, Izaki, and Oka, 2005; Barbero and Mariano, 2006). These studies were motivated by the EFT approach adopted by Parreño, Ramos, and Holstein $(2004,2005)$ where the largest contact term necessary for fitting the weak-decay rates and asymmetries was found to be spin- and isospin-independent; see also the review by Parreño (2007). A careful consideration of scalar-isoscalar two-pion exchange, in terms of a dynamically generated $\sigma$ resonance plus uncorrelated pion exchanges, was shown to resolve the $a_{\Lambda}$ puzzle, as listed in Table XIII, without spoiling the agreement with experimental values of $\Gamma_{\mathrm{nm}}$ and $\Gamma_{n} / \Gamma_{p}$ (Chumillas et al. 2007). In contrast, Itonaga et al. (2008) and Itonaga and Motoba (2010), using perhaps a less microscopic version of $\sigma$-meson degrees of freedom, have claimed that a satisfactory resolution of the $a_{\Lambda}$ puzzle requires a consideration of the axial-vector $a_{1}$, the chiral partner of the $\rho$ meson, in terms of $\rho-\pi$ and $\sigma-\pi$ correlated exchanges. Their results are also listed in Table XIII A similarly small and positive value for ${ }_{\Lambda}^{12} \mathrm{C}, a_{\Lambda}=0.069$, has also been calculated recently by Bauer and Garbarino (2012). 


\section{III. $\Sigma$ HYPERNUCLEI}

\section{A. Overview}

Evidence for relatively narrow $\Sigma$-hypernuclear continuum excitations in ${ }^{6} \mathrm{Li},{ }^{9} \mathrm{Be},{ }^{12} \mathrm{C}$ and ${ }^{16} \mathrm{O}$, with widths of order few $\mathrm{MeV}$, was suggested during the 1980s from $\left(K^{-}, \pi^{ \pm}\right)$experiments in flight at CERN (Bertini et al. 1980, 1984, 1985) and at BNL (Piekarz et al. 1982) using $K^{-}$beams with incident momentum $p_{\text {lab }}=450-720$ $\mathrm{MeV} / \mathrm{c}$, and with stopped $K^{-}$mesons at KEK (Yamazaki et al. 1985). Supporting evidence for $\Sigma$-nuclear attraction, of order $25-30 \mathrm{MeV}$ at central nuclear densities, existed from the "old" analysis of $\Sigma^{-}$atom level shifts and widths (Batty, 1979), but the same analysis also yielded estimates of order $20-30 \mathrm{MeV}$ for the $\Sigma$-nuclear widths at the central nuclear densities expected in $\Sigma$ hypernuclei. The strength of the $\Sigma^{-} p \rightarrow \Lambda n$ reaction, deduced from cross section data at low energies, was shown to be in agreement with this width estimate (Gal and Dover, 1980). In this, and in other calculations (Dover, Millener, and Gal, 1989), the $\Sigma N \rightarrow \Lambda N$ one-pion-exchange transition was perceived to provide the underlying mechanism for $\Sigma$ hypernuclear widths. No sound theoretical calculation was able to reproduce the narrow structures suggested by the reported $\Sigma$ hypernuclear spectra. These spectra, however, typically consisted of a small number of events of questionable statistical significance above the kaon decay background. Subsequent $\left(K^{-}, \pi^{ \pm}\right)$experiments at BNL, with improved statistics, failed to confirm the existence of narrow $\Sigma$ hypernuclear structures (Tang et al., 1988; Bart et al., 1999), particularly on the same targets $\left({ }^{6} \mathrm{Li}\right.$ and $\left.{ }^{9} \mathrm{Be}\right)$ and in the same reactions for which previous claims of quasibound states were made. The new BNL experimental spectra showed somewhat broad continuum enhancements which indicated a very shallow, or even repulsive $\Sigma$ nuclear potential, as had been already argued (Dover, Millener, and Gal, 1989). This was verified by calculations (Dabrowski, 1999) of the pion spectrum in the $\left(K^{-}, \pi^{+}\right)$reaction on ${ }^{9} \mathrm{Be}$ (Bart et al. 1999).

A notable exception is provided by ${ }_{\Sigma}^{4} \mathrm{He}$, where a quasibound state below the $\Sigma^{+}$threshold was discovered in a $\left(K_{\text {stop }}^{-}, \pi^{-}\right)$experiment on ${ }^{4} \mathrm{He}$ at KEK (Hayano et al., 1989). This quasibound state was confirmed in a $\left(K^{-}, \pi^{-}\right)$in-flight experiment, with $p_{\text {lab }}=600 \mathrm{MeV} / \mathrm{c}$, at BNL (Nagae et al. 1998); see Fig. 23. No evidence was found for quasibound states in the companion $\left(K^{-}, \pi^{+}\right)$ experiment on ${ }^{4} \mathrm{He}$. For this reason the ${ }_{\Sigma}^{4} \mathrm{He}$ quasibound state was assigned an isospin value $I=1 / 2$. Comparison of the two spectra in the figure suggests a strong isospin dependence of the $\Sigma$ nuclear potential. This dependence was taken into account in coupled-channel calculations (Harada et al., 1990; Harada, 1998) which used ${ }^{3} \mathrm{He}+\Lambda$, ${ }^{3} \mathrm{He}+\Sigma^{0}$ and ${ }^{3} \mathrm{H}+\Sigma^{+}$channels for $\left(K^{-}, \pi^{-}\right)$and ${ }^{3} \mathrm{H}+\Sigma^{-}$ for $\left(K^{-}, \pi^{+}\right)$. A similar isospin dependence is also clearly

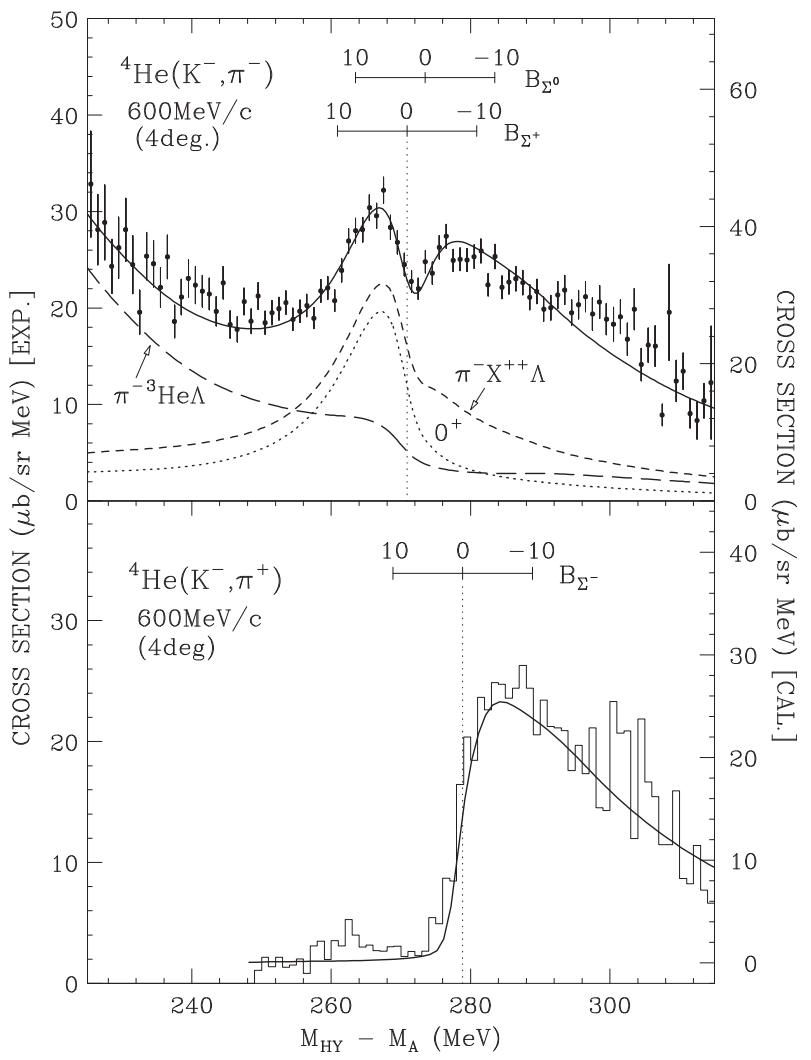

FIG. $23{ }^{4} \mathrm{He}\left(K^{-}, \pi^{ \pm}\right)$spectra measured at BNL (Nagae et al. 1998) and as calculated by Harada (1998), providing evidence for a ${ }_{\Sigma}^{4}$ He $I=1 / 2$ quasibound state in the $\pi^{-}$channel, with fitted values of binding energy $B_{\Sigma^{+}}=4.4 \pm 0.3 \pm 1 \mathrm{MeV}$ and width $\Gamma=7.0 \pm 0.7_{-0.0}^{+1.2} \mathrm{MeV}$. Adapted from Harada, 1998 .

seen in the CERN data (Bertini et al. 1984 ) on ${ }^{12} \mathrm{C}$, as deduced by Dover, Gal, and Millener (1984), and in the BNL measurements (Bart et al. 1999) on ${ }^{6} \mathrm{Li}$ and ${ }^{9} \mathrm{Be}$, as deduced by Dabrowski (1999). The strong isospin dependence may be parameterized in terms of a strong Lane term $V_{1}^{\Sigma}$ of the $\Sigma$ nuclear potential:

$$
V_{\Sigma}(r)=\left(V_{0}^{\Sigma}+\frac{1}{A} V_{1}^{\Sigma} \boldsymbol{T}_{A} \cdot \boldsymbol{t}_{\Sigma}\right) \frac{\rho(r)}{\rho_{0}},
$$

where $\boldsymbol{t}_{\Sigma}$ is the $\Sigma$ isospin operator and $\boldsymbol{T}_{A}$ is the nuclear isospin operator with $\mathrm{z}$ projection $(Z-N) / 2$. Owing to the smallness of $A(A=4)$, the Lane term in the case of ${ }_{\Sigma}^{4} \mathrm{He}$, with a large and positive value of $V_{1}^{\Sigma}$, provides sufficient attraction to generate a quasibound state, whereas the relatively small width is due to the isoscalar repulsion (Harada et al., 1990; Harada, 1998, 2001). A large value, $V_{1}^{\Sigma} \approx 80 \mathrm{MeV}$, had been predicted by Dover, Gal, and Millener, (1984) from the $\left(K^{-}, \pi^{ \pm}\right)$CERN data on ${ }^{12} \mathrm{C}$ (Bertini et al. 1984).

Recent measurements at KEK of the $\Sigma^{-}$spectrum in the $\left(\pi^{-}, K^{+}\right)$reaction on targets across the periodic table (Noumi et al. , 2002, 2003, Saha et al., 2004) have established that the $\Sigma$ nuclear interaction is strongly repulsive. This was subsequently confirmed in DWIA calculations 
TABLE XIV Representative values of isoscalar and isovector $\Sigma$-nuclear potential depths (in MeV) [see Eq. 460] taken from Gal (2010) for Nijmegen soft-core potentials (Rijken, Nagels, and Yamamoto, 2010), and from Haidenbauer and Meißner (2015) for EFT potentials with cutoff parameter $600 \mathrm{MeV}$.

\begin{tabular}{|c|c|c|c|c|c|c|}
\hline & NSC97f & ESC04d & ESC08b & $\mathrm{LO}$ & NLO & Phenomenology \\
\hline$V_{0}^{\Sigma}$ & -13.9 & -26.0 & +20.3 & +22.1 & +14.8 & $+30 \pm 20$ \\
\hline$V_{1}^{\Sigma}$ & -30.4 & +30.4 & +85.2 & +58.1 & +67.8 & $\approx 80$ \\
\hline
\end{tabular}

by Harada and Hirabayashi $(2005,2006)$ as reviewed in Sec. III.C. In parallel, density-dependent analyses of $\Sigma^{-}$atom data in the early 1990s led to the conclusion that the nuclear interaction of $\Sigma$ 's is dominated by repulsion (Batty, Friedman, and Gal, 1994a, 1994b, 1997; Mareš et al., 1995). Based on the various analyses discussed above, A reasonable estimate of the $\Sigma$ isoscalar repulsion, based on the various analyses discussed above, is $V_{0}^{\Sigma} \approx 30 \pm 20 \mathrm{MeV}$, a value listed in Table XIV. The repulsion of $\Sigma^{-}$in nuclear matter, and also in neutron matter, has important repercussions for the balance of strangeness in the inner crust of neutron stars, primarily by delaying to higher densities, or even aborting the appearance of $\Sigma^{-}$hyperons (Balberg and Gal, 1997).

Values of $V_{0}^{\Sigma}$ and $V_{1}^{\Sigma}$ are listed in Table XIV for several representative Nijmegen soft-core potentials and recent EFT calculations, in comparison with phenomenological values derived from several sources of data analyses. Of the hard-core, earlier Nijmegen potentials, only Model F provided isoscalar repulsion and a sizable "attractive" Lane term $\left(V_{1}^{\Sigma}>0\right)$, both of which are required to fit the data, as shown by Dabrowski (1999). For the soft-core Nijmegen models, it is worth noting that the widely used NSC97 models, and the Jülich model (Haidenbauer and Meißner, 2005), produced attractive isoscalar $\Sigma$-nuclear potentials and "repulsive" isovector potentials, just opposite of what phenomenology demands (as marked in the last column of the table). Subsequent Nijmegen potentials have removed this discrepancy by imposing a strongly repulsive $T=3 / 2{ }^{3} S_{1}-{ }^{3} D_{1} \Sigma N$ interaction on their parameter fit. This was motivated by the $\mathrm{SU}(6)$ quark-model, resonating-group method calculations by the Kyoto-Niigata group (Kohno et al. 2000$)$, reviewed by Fujiwara, Suzuki, and Nakamoto (2007), in which a strong Pauli repulsion appears in this $\Sigma N$ channel; see also recent calculations of hyperon-nucleus potentials by Kohno and Fujiwara (2009) and Kohno (2010). The latest EFT potentials, LO (Polinder, Haidenbauer, and Meißner, 2006) and NLO (Haidenbauer et al. 2013), also impose repulsion in this particular $\Sigma N$ channel. An earlier SU(3) chiral perturbation calculation by Kaiser (2005) yielded repulsion of order $V_{0}^{\Sigma} \approx 60 \mathrm{MeV}$.

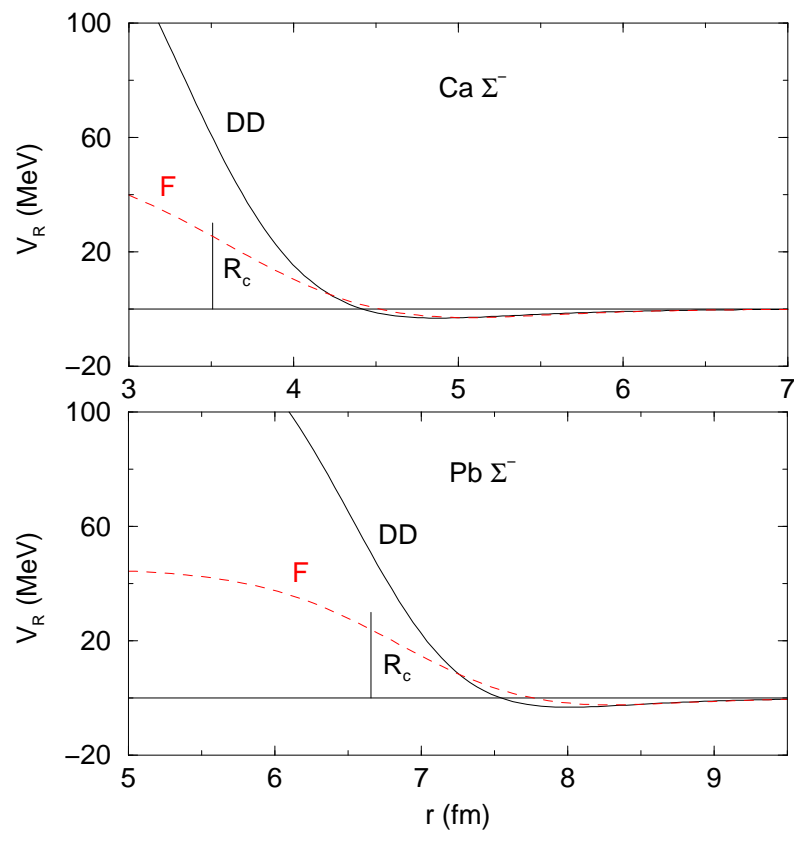

FIG. 24 Re $V_{\text {opt }}\left(V_{R}\right)$ for two different parameterizations of the $\Sigma^{-}$nuclear potential, DD (solid) and F (dashed), fitted to $\Sigma^{-}$atomic data. Vertical bars indicate the half-density radius of the nuclear charge distribution. From Friedman and Gal, 2007.

\section{B. $\Sigma$ nuclear potentials from fits to $\Sigma^{-}$atoms}

$\Sigma^{-}$nuclear potentials resulting from two fits to the full set of $\Sigma^{-}$atomic data, with different parameterizations for the density dependence of $V_{\Sigma}$, are shown in Fig. 24. The data consist of 23 strong-interaction level shifts, widths, and yields. A phenomenological densitydependent isoscalar potential, DD, was introduced by the form (Batty, Friedman, and Gal, 1994a, 1994b)

$$
V_{\Sigma}(r) \sim\left\{b_{0}+B_{0}[\rho(r) / \rho(0)]^{\alpha}\right\} \rho(r), \quad \alpha>0
$$

and a "geometrical" potential, F, was introduced by the form (Mareš, Friedman, and Gal, 2006)

$$
V_{\Sigma}(r) \sim\left\{b_{0}[1-F(r)]+B_{0} F(r)\right\} \rho(r) .
$$

In these expressions

$$
F(r)=\frac{1}{e^{x}+1}, \quad x=\frac{r-R_{x}}{a_{x}},
$$

with $R_{x}=R_{x 0} A^{1 / 3}+\delta_{x}$ close to the radius of the nucleus, and $a_{x} \approx 0.5 \mathrm{fm}$ close to accepted values of the nuclear diffusivity. Greatly improved fits with respect to fitted $t \rho(r)$ type potentials are obtained by fitting the parameters $b_{0}, B_{0}$, and $\alpha$, for $\mathrm{DD}$, and $b_{0}, B_{0}$, and $R_{x 0}, \delta_{x}, a_{x}$, for $\mathrm{F}$. Isovector components are readily included, but are found to have a marginal effect. The fit to the data is equally good in the two models, with a $\chi^{2}$ per degree of 
freedom of 1.0 for DD and 0.9 for F. The half-density radius of the charge distribution $R_{c}$ is indicated in Fig. 24 . The figure demonstrates that the transition from outward attraction to inward repulsion occurs well outside $R_{c}$, a property supported also by other types of fits to $\Sigma^{-}$atomic data (Friedman and Gal, 2007). The precise magnitude and shape of the repulsive component within the nucleus is not determined by the $\Sigma^{-}$atomic data. Although both models show weak attraction at large radii, this is too weak to support bound states. The conclusion is in agreement with the experimental results from BNL (Bart et al. 1999) showing the absence of $\Sigma$ hypernuclear quasibound peaks beyond He.

\section{Evidence from $\left(\pi^{-}, K^{+}\right)$spectra}

More straightforward information on the nature of the $\Sigma$-nuclear interaction has been provided by recent measurements of inclusive $\left(\pi^{-}, K^{+}\right)$spectra on medium to heavy nuclear targets at KEK (Noumi et al., 2002 , 2003 Saha et al., 2004). These spectra were fitted using Woods-Saxon potentials with depths $V_{0} \approx 100 \mathrm{MeV}$ for the repulsive real part and $W_{0}=-40 \mathrm{MeV}$ for the imaginary part. There is less sensitivity to the imaginary (absorptive) component. The repulsive potential in this analysis is of the same order of magnitude as obtained for the DD potential in the nuclear surface region, Fig. 24

More sophisticated theoretical analyses of these KEK $\left(\pi^{-}, K^{+}\right)$spectra (Kohno et al., 2004, 2006; Harada and Hirabayashi, 2005, 2006) have also concluded that the $\Sigma$-nuclear potential is repulsive within the nuclear volume, although they yield a weaker repulsion in the range of $10-40 \mathrm{MeV}$. An example of a recent analysis of the Si spectrum is shown in Fig. 25 from Harada and Hirabayashi (2005), where six different $\Sigma$-nucleus potentials are tested for their ability to reproduce the measured ${ }^{28} \operatorname{Si}\left(\pi^{-}, K^{+}\right)$spectrum (Saha et al., 2004) within the DWIA. This particular DWIA version was tested on the well-understood ${ }^{28} \mathrm{Si}\left(\pi^{+}, K^{+}\right)$quasifree $\Lambda$ hypernuclear spectrum which was also taken at KEK with pions of the same incident momentum, $p_{\text {lab }}=1.2 \mathrm{GeV} / \mathrm{c}$. The potential of Fig. 25(a) is the DD, type $\mathrm{A}^{\prime}$ potential of Batty, Friedman, and Gal (1994a, 1994b), that of Fig. 25(b) is one of the RMF potentials of Mareš et al. (1995), with $\alpha_{\omega}=1$, and that of Fig. 25(c) is a local-density approxiamation version of a $G$ matrix constructed from the Nijmegen model F. These three potentials are repulsive within the nucleus but differ considerably from each other. The potentials of Figs. 25(d)-(f) are all attractive within the nucleus, with that of of Fig. 25(f) being of a t $\rho$ form. All of the six potentials are attractive outside the nucleus, as required by fits to the "attractive" $\Sigma^{-}$atomic level shifts. The figure shows clearly that fully attractive potentials are ruled out by the data, as deduced from $\chi^{2}$ fits, and that only the "repulsive" $\Sigma$-nucleus potentials reproduce the spectrum, although without preference to any of these repulsive potentials.

\section{IV. $\Lambda-\Lambda$ HYPERNUCLEI}

Until 2001 only three emulsion events had been considered serious candidates for $\Lambda \Lambda$ hypernuclei: ${ }_{\Lambda \Lambda}^{10} \mathrm{Be}$ (Danysz et al., 1963a, 1963b), ${ }_{\Lambda \Lambda}^{6} \mathrm{He}$ (Prowse, 1966) and ${ }_{\Lambda \Lambda}^{13} \mathrm{~B}$ Aoki et al. 1991 . The $\Lambda \Lambda$ binding energies deduced from these emulsion events indicated that the $\Lambda \Lambda$ interaction was quite attractive in the ${ }^{1} S_{0}$ channel (Dalitz et al., 1989; Dover et al., 1991; Yamamoto, Takaki, and Ikeda, 1991), with a $\Lambda \Lambda$ excess binding energy $\Delta B_{\Lambda \Lambda} \sim 4.5 \mathrm{MeV}$. However, it was realized that the binding energies of ${ }_{\Lambda \Lambda}^{10} \mathrm{Be}$ and ${ }_{\Lambda \Lambda}^{6} \mathrm{He}$ were inconsistent with each other (Bodmer, Usmai, and Carlson, 1984; Wang, Takaki, and Bandō, 1986). Here, the $\Lambda \Lambda$ excess binding energy is defined by

$$
\Delta B_{\Lambda \Lambda}\left({ }_{\Lambda \Lambda}^{A} Z\right)=B_{\Lambda \Lambda}\left({ }_{\Lambda \Lambda}^{A} Z\right)-2 \bar{B}_{\Lambda}\left({ }_{\Lambda}^{(A-1)} Z\right),
$$

where $B_{\Lambda \Lambda}\left({ }_{\Lambda \Lambda}^{A} Z\right)$ is the $\Lambda \Lambda$ binding energy of the hypernucleus ${ }_{\Lambda \Lambda}^{A} Z$ and $\bar{B}_{\Lambda}\left({ }_{\Lambda}^{(A-1)} Z\right)$ is the $(2 J+1)$-average of $B_{\Lambda}$ values for the ${ }_{\Lambda}^{(A-1)} Z$ hypernuclear core levels in the g.s. doublet, as appropriate to a spin-zero $\left(1 s_{\Lambda}\right)^{2}$ configuration of the double- $\Lambda$ hypernucleus ${ }_{\Lambda \Lambda}^{A} Z$. The unambiguous observation of ${ }_{\Lambda \Lambda}^{6} \mathrm{He}$ (Takahashi et al. , 2001) from the KEK hybrid-emulsion experiment E373 lowered the accepted $\Delta B_{\Lambda \Lambda}$ value substantially from the value deduced from the older, dubious event (Prowse, 1966), down to $\Delta B_{\Lambda \Lambda}\left({ }_{\Lambda \Lambda}^{6} \mathrm{He}\right)=0.67 \pm 0.17 \mathrm{MeV}$ (Ahn et al. 2013). With this new value of $\Delta B_{\Lambda \Lambda}$, it is natural to inquire where the onset of $\Lambda \Lambda$ binding occurs. From the very beginning it was recognized that the $\Lambda \Lambda$ system (Dalitz, 1963b) and the three-body $\Lambda \Lambda N$ system were unbound (Tang and Herndon, 1965); if $\Lambda \Lambda N$ were bound, the existence of a bound $n n \Lambda$ would follow and ${ }_{\Lambda \Lambda}^{6} \mathrm{He}$ would most likely become overbound (Gal, 2013). The existence of a ${ }_{\Lambda \Lambda}^{4} \mathrm{H}$ bound state was claimed by the AGS experiment E906 (Ahn et al. 2001b), studying correlated weak-decay pions emitted sequentially from $\Lambda \Lambda$ hypernuclei apparently produced in a $\left(K^{-}, K^{+}\right)$reaction on ${ }^{9} \mathrm{Be}$, but this interpretation is ambiguous (Randeniya and Hungerford, 2007).

The issue of ${ }_{\Lambda \Lambda}^{4} \mathrm{H}$ binding was addressed in several subsequent studies. A Faddeev-Yakubovsky (FY) four-body calculation (Filikhin and Gal, 2002b found no bound state when using an $s$-wave $V_{\Lambda \Lambda}$ fitted to $B_{\Lambda \Lambda}\left({ }_{\Lambda \Lambda}^{6} \mathrm{He}\right)$ and a $V_{\Lambda N}$ partially fitted to $B_{\Lambda}\left({ }_{\Lambda}^{3} \mathrm{H}\right)$. However, when fitting a $\Lambda d$ potential to the low-energy parameters of the $s$-wave Faddeev calculation for $\Lambda p n$ and solving the $s$-wave Faddeev equations for a $\Lambda \Lambda d$ model of ${ }_{\Lambda \Lambda}^{4} \mathrm{H}$, a $1^{+}$ bound state was obtained. Disregarding spin it can be shown, for essentially an attractive $\Lambda \Lambda$ interaction and for a static nuclear core $d$, that a two-body $\Lambda d$ bound state 


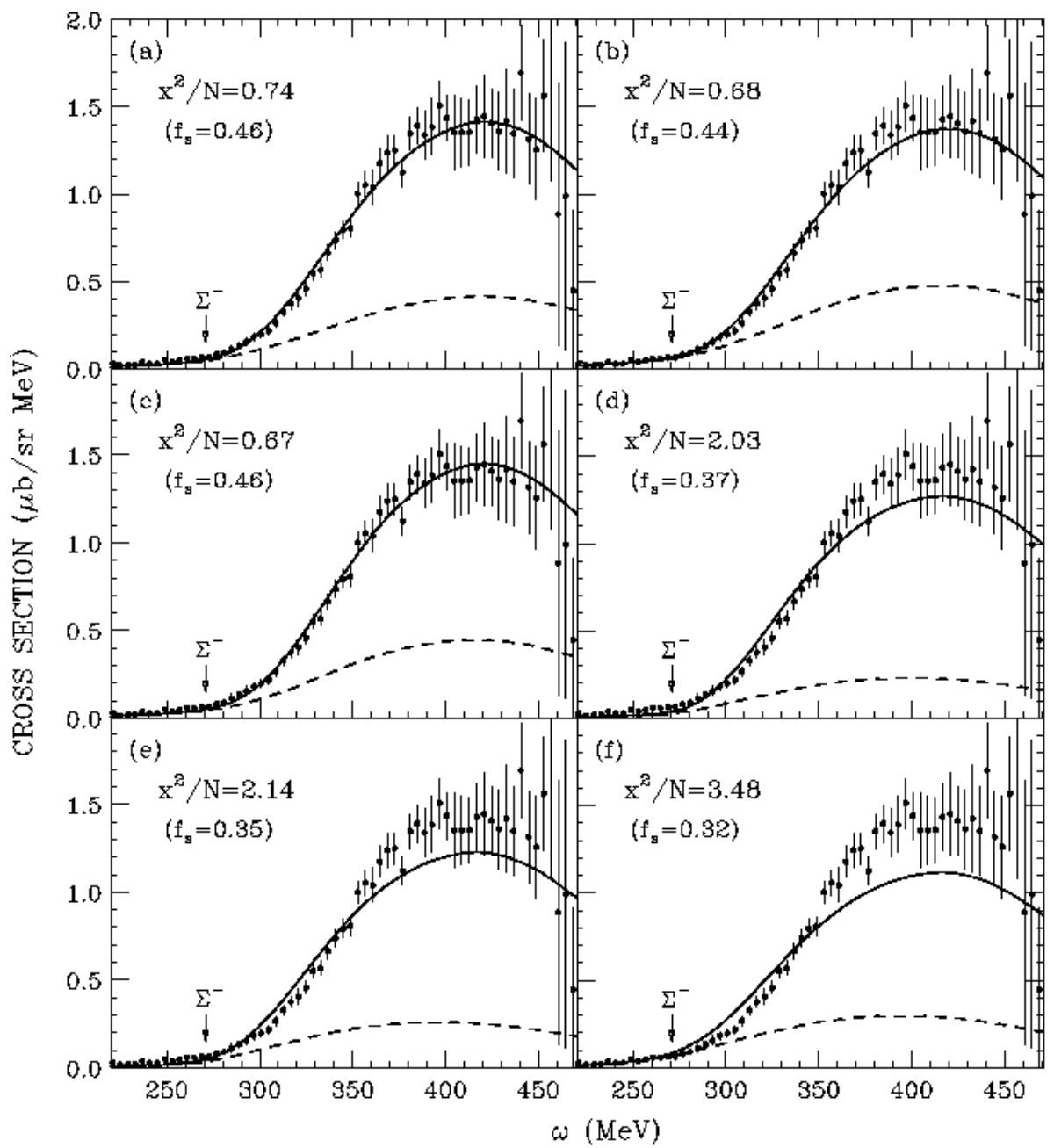

FIG. 25 Comparison between DWIA calculations (Harada and Hirabayashi 2005, 2006) using six $\Sigma$-nucleus potentials, (a)-(c) with inner repulsion, (d)-(f) fully attractive, and the measured ${ }^{28} \mathrm{Si}\left(\pi^{-}, K^{+}\right)$spectrum (Saha et al. 2004). The solid and dashed curves denote the inclusive and $\Lambda$ conversion cross sections, respectively. Each calculated spectrum was normalized by a fraction $f_{s}$. The arrows mark the $\Sigma^{-}-{ }^{27} \mathrm{Al}$ (g.s.) threshold at $\omega=270.75 \mathrm{MeV}$. From Harada and Hirabayashi, 2005.

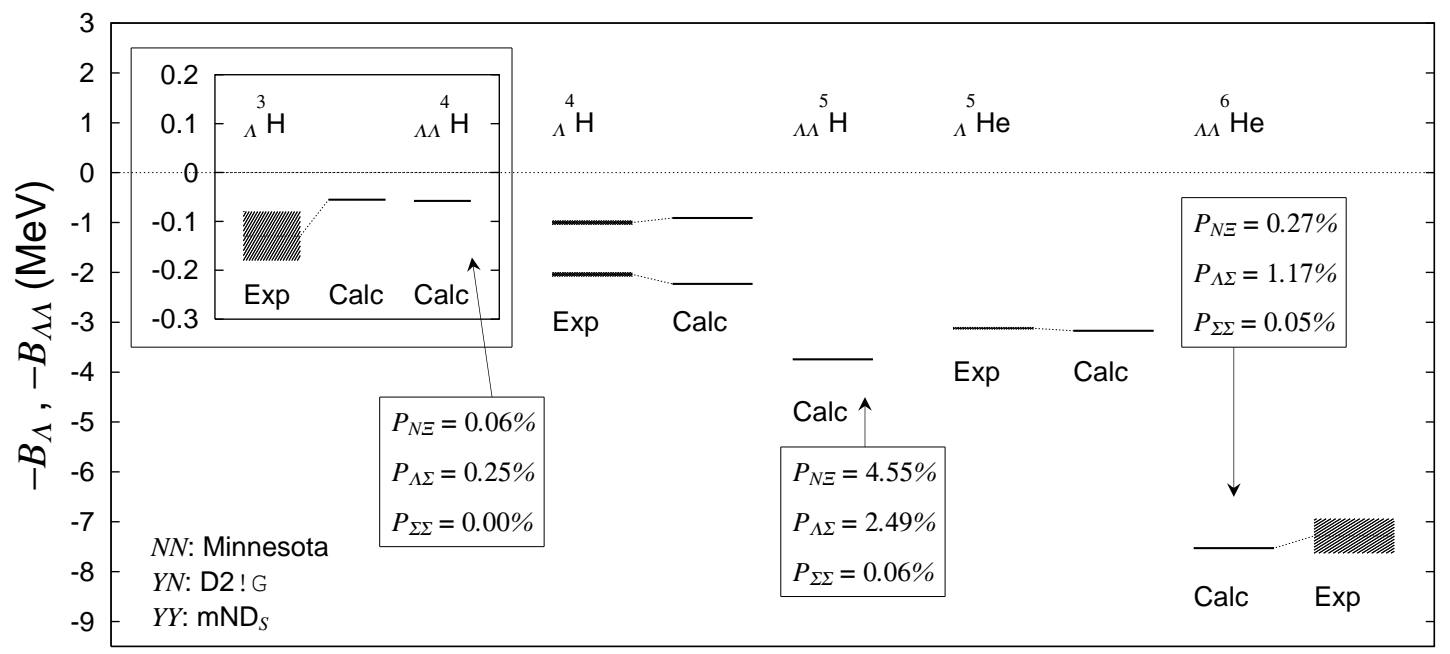

FIG. 26 Calculated $\Lambda$ and $\Lambda \Lambda$ separation energies of $s$-shell hypernuclei. From Nemura et al., 2005. 


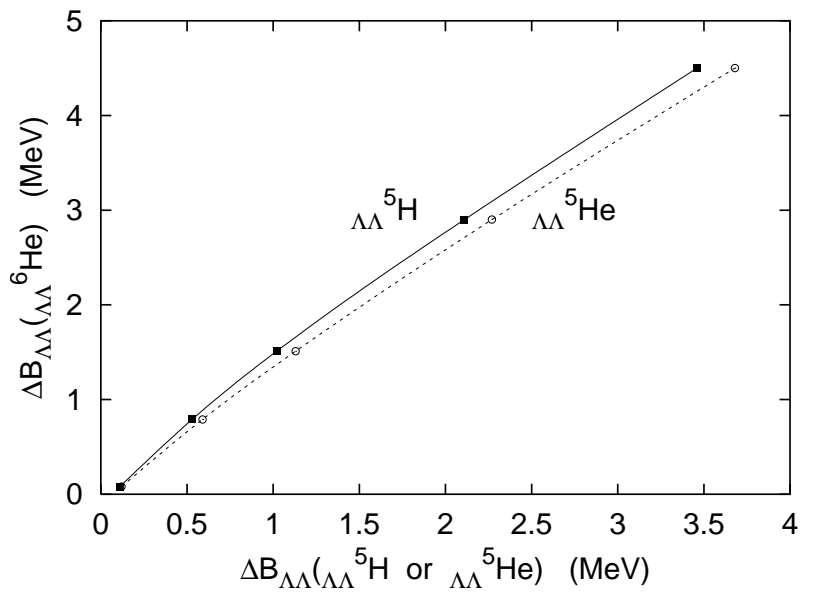

FIG. 27 Faddeev calculations of $\Delta B_{\Lambda \Lambda}$ for ${ }_{\Lambda \Lambda}^{6} \mathrm{He}$ vs. Faddeev calculations for the mirror $\Lambda \Lambda$ hypernuclei ${ }_{\Lambda}{ }^{5} \mathrm{H}$ and ${ }_{\Lambda}{ }_{\Lambda}^{5} \mathrm{He}$. The points mark results obtained for various assumptions on $V_{\Lambda \Lambda}$. From Filikhin and Gal, 2002a

implies binding for the three-body $\Lambda \Lambda d$ system. Nevertheless, for a non-static nuclear core $d$ (made of a pn interacting pair), a $\Lambda d$ bound state does not necessarily imply binding for the $\Lambda \Lambda d$ system.

This ${ }_{\Lambda \Lambda}^{4} \mathrm{H}$ no-binding conclusion was challenged by Nemura, Akaishi, and Myint (2003) and Nemura et al. (2005) who showed that $\Lambda N-\Sigma N$ coupling, which is so important for the quantitative discussion of light $\Lambda$ hypernuclei, is capable of inducing appreciable $\Xi N$ admixures into light $\Lambda \Lambda$ hypernuclei via the $\Sigma \Lambda-\Xi N$ coupling. This is shown in Fig. 26 along with all other bound $\Lambda$ and $\Lambda \Lambda s$-shell hypernuclei. Although in their calculation the second $\Lambda$ in ${ }_{\Lambda \Lambda}^{4} \mathrm{H}$ is bound by $0-0.07 \mathrm{MeV}$, no firm conclusion can be made regarding the particle stability of this species since in their ${ }_{\Lambda \Lambda}^{6} \mathrm{He}$ calculation the second $\Lambda$ is overbound by $0.22 \mathrm{MeV}$. Thus, the issue of the onset of $\Lambda \Lambda$ binding, in particular whether or not ${ }_{\Lambda \Lambda}^{4} \mathrm{H}$ is particle stable, is still unresolved. Further experimental work is needed to decide whether the events reported in the AGS experiment E906 correspond to ${ }_{\Lambda \Lambda}^{4} \mathrm{H}$ (Ahn et al. 2001b; Randeniya and Hungerford, 2007), and also in view of subsequent conflicting theoretical analyses (Kumagai-Fuse and Okabe, 2002; Kahana, Kahana, and Millener, 2003).

Regardless of whether ${ }_{\Lambda \Lambda}^{4} \mathrm{H}$ is particle-stable or not, there is a general consensus that the mirror $\Lambda \Lambda$ hypernuclei ${ }_{\Lambda \Lambda}^{5} \mathrm{H}$ and ${ }_{\Lambda \Lambda}^{5} \mathrm{He}$ are particle-stable, with $\Delta B_{\Lambda \Lambda} \sim$ 0.5-1 MeV (Filikhin and Gal, 2002a; Filikhin, Gal, and Suslov, 2003; Lanskoy and Yamamoto, 2004; Nemura et al., 2005). This is demonstrated in Fig. 27 where calculated $\Delta B_{\Lambda \Lambda}(A=5)$ values, for several potentials $V_{\Lambda \Lambda}$ with different strengths, are shown to be correlated with calculated $\Delta B_{\Lambda \Lambda}(A=6)$ values. A minimum value of $\Delta B_{\Lambda \Lambda}(A=5) \approx 0.1$ is seen to be required for getting $\Delta B_{\Lambda \Lambda}(A=6)>0$, and for the actual value of $\Delta B_{\Lambda \Lambda}(A=6)=0.67 \pm 0.17 \mathrm{MeV}$ the $A=5 \Lambda \Lambda$ hy- pernuclei come out safely bound. It was also argued that $\Lambda \Lambda-\Xi N$ coupling is particularly important for the binding of the $A=5 \Lambda \Lambda$ hypernuclei, increasing $\Delta B_{\Lambda \Lambda}$ for these systems above the corresponding value of $1 \mathrm{MeV}$ in ${ }_{\Lambda \Lambda}^{6} \mathrm{He}$, with the Nijmegen model ESC04d giving as much as $2 \mathrm{MeV}$ (Yamamoto and Rijken, 2008). In addition, substantial charge-symmetry breaking effects are expected in these systems, resulting in a higher binding energy of ${ }_{\Lambda \Lambda}^{5} \mathrm{He}$ by up to $0.5 \mathrm{MeV}$ with respect to ${ }_{\Lambda \Lambda}^{5} \mathrm{H}$ (Lanskoy and Yamamoto, 2004, Yamamoto and Rijken, 2008).

Whereas the assignment of ${ }_{\Lambda \Lambda}^{6} \mathrm{He}$ to the KEK E373 emulsion event (Takahashi et al. 2001) is unique, because it has no particle-stable excited states and the daughter ${ }_{\Lambda}^{5}$ He hypernucleus has no particle-stable excited states to be formed in sequential $\pi^{-}$weak decays, the assignment of other, heavier $\Lambda \Lambda$ hypernuclei to the few emulsion events reported by the KEK E176 and KEK E373 experiments is plagued by ambiguities resulting from the presence of particle-stable excited states in which a $\Lambda \Lambda$ hypernucleus may be formed or to which it may weakly decay. In fact, the $B_{\Lambda \Lambda}^{\exp }$ value listed in Table XV for the KEK E373 Demachi-Yanagi event (Ahn et al. 2001a) assumes that ${ }_{\Lambda \Lambda}^{10} \mathrm{Be}$ was formed in its $2^{+}$first excited state (Filikhin and Gal, 2002a, Hiyama et al., 2002), whereas the earlier observation of ${ }_{\Lambda \Lambda}^{10} \mathrm{Be}$ (Danysz et al., $\left.1963 \mathrm{~b}\right)$ was interpreted as involving the weak decay of ${ }_{\Lambda \Lambda}^{10} \mathrm{Be}_{\text {g.s. }}$ to the excited doublet levels $\left(3 / 2^{+}, 5 / 2^{+}\right)$in ${ }_{\Lambda}^{9}$ Be (Danysz et al. 1963a). The $\approx 3 \mathrm{MeV}$ unobserved $\gamma$-ray de-excitation energy has to be accounted for in each one of these scenarios, and the $\approx 6 \mathrm{MeV}$ difference between the $B_{\Lambda \Lambda}^{\exp }$ values originally claimed for these two events of ${ }_{\Lambda \Lambda}^{10} \mathrm{Be}$ is consistent $(6=3+3)$ with the reinterpretations offered here. Other scenarios, involving production neutrons or decay neutrons which are unobserved in emulsion, have also been considered (Davis, 2005). Similarly, the $B_{\Lambda \Lambda}^{\exp }$ value assigned in the table to ${ }_{\Lambda \Lambda}^{13} \mathrm{~B}$ also assumes an unobserved $\gamma$ ray $E_{\gamma} \approx 4.8 \mathrm{MeV}$ from the electromagnetic decay of the excited doublet levels $\left(3 / 2^{+}, 5 / 2^{+}\right)$in ${ }_{\Lambda}^{13} \mathrm{C}$ formed in the weak decay ${ }_{\Lambda \Lambda}^{13} \mathrm{~B} \rightarrow{ }_{\Lambda}^{13} \mathrm{C}\left(3 / 2^{+}, 5 / 2^{+}\right)$.

Table XV provides a comprehensive listing of candidate $\Lambda \Lambda$-hypernuclear emulsion events, along with $\Lambda \Lambda$ binding energy values derived from these events, with caveats explained earlier for ${ }_{\Lambda \Lambda}^{10} \mathrm{Be}$ and ${ }_{\Lambda \Lambda}^{13} \mathrm{~B}$. The table also lists calculated $\Lambda \Lambda$ binding energies using (i) fewbody cluster models (Hiyama et al. 2002,2010$)$, and (ii) shell-model evaluations (Gal and Millener, 2011). The table makes it clear that the shell-model methodology is able to confront any of the reported $\Lambda \Lambda$ species, whereas cluster models have been limited so far to 3-,4- and 5body calculations. For those $\Lambda \Lambda$ hypernuclei where a comparison between the two models is possible, the calculated binding energies are remarkably close to each other. The shell-model (SM) estimate for $B_{\Lambda \Lambda}$ in the nuclear $p$ 
TABLE XV $B_{\Lambda \Lambda}$ values (in MeV) from KEK experiments E176 (Aoki et al., 2009) and E373 (Ahn et al. 2013), and as calculated in cluster models (Hiyama et al. 2002,2010 ) and in the shell model (Gal and Millener, 2011). $B_{\Lambda \Lambda}\left(\Lambda \Lambda{ }^{\circ} \mathrm{He}\right)$ serves as input in both types of calculations. The E176 entries offer several assignments to the same single emulsion event observed.

\begin{tabular}{lccccc}
\hline \hline Event & ${ }_{\Lambda \Lambda}^{\mathrm{A}} \mathrm{Z}$ & $\bar{B}_{\Lambda}\left({ }_{\Lambda}^{\mathrm{A}-1} \mathrm{Z}\right)$ & $B_{\Lambda \Lambda}^{\exp }$ & $B_{\Lambda \Lambda}^{\mathrm{CM}}$ & $B_{\Lambda \Lambda}^{\mathrm{SM}}$ \\
\hline E373-Nagara & ${ }_{\mathrm{6}}^{6} \mathrm{He}$ & $3.12 \pm 0.02$ & $6.91 \pm 0.16$ & $6.91 \pm 0.16$ & $6.91 \pm 0.16$ \\
E373-DemYan & ${ }_{\Lambda \Lambda} \mathrm{Be}$ & $6.71 \pm 0.04$ & $14.94 \pm 0.13$ & $14.74 \pm 0.16$ & $14.97 \pm 0.22 \mathrm{a}$ \\
E176-G2 & ${ }_{1 \Lambda} \mathrm{Be}$ & $8.86 \pm 0.11$ & $17.53 \pm 0.71$ & $18.23 \pm 0.16$ & $18.40 \pm 0.28$ \\
E373-Hida & ${ }_{\Lambda \Lambda} \mathrm{Be}$ & $8.86 \pm 0.11$ & $20.83 \pm 1.27$ & $18.23 \pm 0.16$ & $18.40 \pm 0.28$ \\
E373-Hida & ${ }_{1 \Lambda} \mathrm{Be}$ & $10.02 \pm 0.05$ & $22.48 \pm 1.21$ & - & $20.72 \pm 0.20$ \\
E176-E2 & ${ }_{12} \mathrm{~B}$ & $10.09 \pm 0.05$ & $20.02 \pm 0.78$ & - & $20.85 \pm 0.20$ \\
E176-E4 & ${ }_{\Lambda \Lambda}^{13} \mathrm{~B}$ & $11.27 \pm 0.06$ & $23.4 \pm 0.7$ & - & $23.21 \pm 0.21$ \\
\hline \hline
\end{tabular}

${ }^{\text {a }} B_{\Lambda \Lambda}^{\mathrm{SM}}\left({ }_{\Lambda \Lambda}^{10} \mathrm{Be}\right)=2 \bar{B}_{\Lambda}\left({ }_{\Lambda}^{9} \mathrm{Be}\right)+4\left[\bar{V}\left({ }_{\Lambda}^{9} \mathrm{Be}\right)-\bar{V}_{\text {average }}\right]+\left\langle V_{\Lambda \Lambda}\right\rangle_{\mathrm{SM}}$, see Eq. 52.

shell is given simply by

$$
B_{\Lambda \Lambda}^{\mathrm{SM}}\left({ }_{\Lambda \Lambda}^{\mathrm{A}} \mathrm{Z}\right)=2 \bar{B}_{\Lambda}\left({ }_{\Lambda}^{\mathrm{A}-1} \mathrm{Z}\right)+\left\langle V_{\Lambda \Lambda}\right\rangle_{\mathrm{SM}},
$$

where $\left\langle V_{\Lambda \Lambda}\right\rangle_{\mathrm{SM}}$ is a $\Lambda \Lambda$ interaction matrix element identified with $\Delta B_{\Lambda \Lambda}\left({ }_{\Lambda}{ }_{\Lambda} \mathrm{He}\right)=0.67 \pm 0.17 \mathrm{MeV}$. In cluster-model (CM) calculations (Hiyama et al., 2010), $\left\langle V_{\Lambda \Lambda}\right\rangle_{\mathrm{CM}} \equiv B_{\Lambda \Lambda}\left(V_{\Lambda \Lambda} \neq 0\right)-B_{\Lambda \Lambda}\left(V_{\Lambda \Lambda}=0\right)$ assumes similar values: $0.54,0.53$ and $056 \mathrm{MeV}$ for ${ }_{\Lambda \Lambda}^{6} \mathrm{He},{ }_{\Lambda \Lambda}^{10} \mathrm{Be}$ and ${ }_{\Lambda \Lambda}^{11} \mathrm{Be}$, respectively. To apply Eq. $\left[51, \bar{B}_{\Lambda}\left({ }_{\Lambda}{ }_{\Lambda} \mathrm{Z}\right)\right.$ is derived from the shell-model calculations outlined in Sec. II.A.2 on $p$-shell single- $\Lambda$ hypernuclei. Apart from the spin dependence of the $\Lambda N$ interaction, which is fully constrained by the $\gamma$-ray measurements and their shellmodel analyses, the validity of a uniform shell-model description of hypernuclei throughout the whole $p$ shell depends on the constancy of the $\Lambda N$ spin-independent matrix element $\bar{V}$ in the mass range considered. Indeed, excluding ${ }_{\Lambda}^{9} \mathrm{Be}$ which deviates substantially from the other species, a common value $\bar{V}^{\mathrm{SM}}=-1.06 \pm 0.03 \mathrm{MeV}$ can be assigned. In ${ }_{\Lambda}^{9} \mathrm{Be}$, the $\Lambda$ hyperon is attached to a somewhat loose $\alpha-\alpha$ structure, but in ${ }_{\Lambda \Lambda}^{10}$ Be the second $\Lambda$ is bound with respect a normal ${ }_{\Lambda}^{5} \mathrm{He}-\alpha$ structure. This suggests an extension of the validity of Eq. (51) also to ${ }_{\Lambda \Lambda}^{10} \mathrm{Be}$ by adding to its right-hand side a correction term $\delta B_{\Lambda \Lambda}^{\mathrm{SM}}$ due to the normally bound second $\Lambda$ :

$$
\delta B_{\Lambda \Lambda}^{\mathrm{SM}}\left({ }_{\Lambda \Lambda}^{\mathrm{A}} \mathrm{Z}\right)=(A-6)\left[\bar{V}\left(\begin{array}{c}
\mathrm{A}-1 \\
\Lambda
\end{array}\right)-\bar{V}^{\mathrm{SM}}\right]
$$

where $\Lambda-\Sigma$ contributions $\lesssim 0.1 \mathrm{MeV}$ were disregarded. Cluster models, on the other hand, are able to treat the ${ }^{8} \mathrm{Be}$ core in terms of a loose $\alpha-\alpha$ structure, as well as ${ }_{\Lambda}^{9} \mathrm{Be}$ and ${ }_{\Lambda \Lambda}^{10} \mathrm{Be}$ as $\alpha \alpha n$ and $\alpha \alpha n n$ clusters, respectively, but they encounter difficulties in consistently evaluating spin-dependent $\Lambda N$ interaction contributions.

Inspection of Table $\mathrm{XV}$ shows that the binding energies of both ${ }_{\Lambda \Lambda}^{10} \mathrm{Be}$ and ${ }_{\Lambda \Lambda}^{13} \mathrm{~B}$ are well reproduced by the shell model, thereby confirming the interpretations of the corresponding emulsion events discussed earlier. Of the other $\Lambda \Lambda$ hypernuclear candidates, the E373-Hida event (Ahn et al., 2013) does not fit any reasonable assignment as ${ }_{\Lambda \Lambda}^{11} \mathrm{Be}$ or ${ }_{\Lambda \Lambda}^{12} \mathrm{Be}$. Regarding the species listed in the table as due to E176, they all correspond to different assignments of the same event, for which the ${ }_{\Lambda \Lambda}^{13} \mathrm{~B}$ assignment is statistically preferable (Aoki et al., 2009).

\section{V. $\Xi$ HYPERNUCLEI}

Very little is established experimentally or phenomenologically on the interaction of $\Xi$ hyperons with nuclei. Dover and Gal (1983), analyzing old emulsion data which were interpreted as due to $\Xi^{-}$hypernuclei, obtained an attractive $\Xi$-nucleus interaction with a nuclear potential well depth of $-V_{0}^{\Xi}=21-24 \mathrm{MeV}$. This range of values agreed well with the theoretical prediction (Dover and Gal, 1984) for $\Xi$ in nuclear matter, using the early hard-core model D of the Nijmegen group (Nagels, Rijken, and de Swart, 1977) to describe baryon-baryon interactions in a $\mathrm{SU}(3)_{\mathrm{f}}$ framework. However, this is in contrast with the $\Xi$-nucleus repulsion obtained using the other hard-core model, model F (Nagels, Rijken, and de Swart, 1979). Predictions made subsequently using more detailed $G$-matrix studies (Yamamoto et al., 1994; Yamamoto, 1995a, 1995b) spanned a whole range of $\Xi$ nucleus well depths by varying the hard-core radius in these Nijmegen models. The confidence in the predictive power of model $\mathrm{D}$ in strangeness -2 hypernuclear physics was due, to a large extent, to its success in yielding the substantial attractive $\Lambda \Lambda$ interaction that was deemed necessary to reproduce the three known $\Lambda \Lambda$ binding energies before 2001. This picture has changed since then for several reasons.

- Inclusive $\left(K^{-}, K^{+}\right)$spectra taken at KEK and at BNL on ${ }^{12} \mathrm{C}$ (Fukuda et al. 1998, Khaustov et al. 2000a) yield more moderate values for the attractive $\Xi$ well depth, $-V_{0}^{\Xi} \sim 15 \mathrm{MeV}$ when fitted near the $\Xi^{-}$-hypernuclear threshold.

- The uniquely identified ${ }_{\Lambda}^{6} \mathrm{He}$ hypernucleus (Takahashi et al., 2001) implies a considerably weaker $\Lambda \Lambda$ interaction than produced by the original version of the Nijmegen hard-core Model D. The 
TABLE XVI Isoscalar, $V_{0}^{\Xi}$, and isovector, $V_{1}^{\Xi}, \Xi$ nuclearmatter potential depths, and widths $\Gamma_{\Xi}$, all in $\mathrm{MeV}$, in recent extended soft core (ESC) Nijmegen potentials, ESC04 (Rijken and Yamamoto, 2006b) and ESC08 (Nagels, Rijken, and Yamamoto, 20015a).

\begin{tabular}{cccc}
\hline \hline Potential & $V_{0}^{\Xi}$ & $V_{1}^{\Xi}$ & $\Gamma_{\Xi}$ \\
\hline ESC04d & -18.7 & +50.9 & 11.4 \\
ESC08c & -7.0 & +21.6 & 4.5 \\
\hline \hline
\end{tabular}

Nijmegen soft-core potentials NSC97 (Stoks and Rijken, 1999) and extended soft-core potentials ESC04 (Rijken and Yamamoto, 2006b) provide a more realistic framework for the weaker $\Lambda \Lambda$ interaction. The NSC97 potentials slightly underestimate $\Delta B_{\Lambda \Lambda}\left({ }_{\Lambda \Lambda}^{6} \mathrm{He}\right)$, whereas the ESC04 potentials overestimate it, occasionally by about $0.5 \mathrm{MeV}$, and the ESC08 potentials only by up to $0.3 \mathrm{MeV}$ (Yamamoto, Motoba, and Rijken, 2010).

Representative values of isoscalar, $V_{0}^{\Xi}$, and isovector, $V_{1}^{\Xi}, \Xi$ potential depths and width, $\Gamma_{\Xi}$, from $G$-matrix calculations at nuclear-matter density $\left(k_{F}=1.35 \mathrm{fm}^{-1}\right)$ using the Nijmegen extended soft-core models ESC04d and ESC08c, are listed in Table XVI. The isovector (Lane) potential $V_{1}^{\Xi}$ is defined by Eq. (46) where $\boldsymbol{t}_{\Sigma}$ is replaced by $\boldsymbol{t}_{\Xi}$. The isoscalar potential comes out repulsive in ESC04a,b and attractive in ESC04c, d, whereas it is attractive in all ESC08 versions. The focus in Table XVI on attractive $\Xi$-nucleus isoscalar potentials, $V_{0}^{\Xi}<0$, is motivated by the experimental hints from KEK (Fukuda et al. 1998) and BNL (Khaustov et al. 2000a) mentioned above. Both ESC04d and ESC08c $\Xi N$ potentials are attractive in the isospin $I=0,1{ }^{3} S_{1}-{ }^{3} D_{1}$ channels, which might lead to $\Xi N$ bound states, while the ${ }^{1} S_{0}$ channels are repulsive. The models give rise to a positive isovector potential depth $V_{1}^{\Xi}$. The predictions of spin-flavor $\mathrm{SU}(6)$ quark models (Fujiwara, Kohno, and Suzuki, 2007; Fujiwara, Suzuki, and Nakamoto, 2007) differ in detail, but the overall picture for the isoscalar $\Xi$-nuclear potential depths is similar, with a slightly attractive isoscalar potential, $V_{0}^{\Xi}<0$, and a positive isovector potential depth, $V_{1}^{\Xi}>0$. In both approaches, however, the $\Xi-\alpha$ system will not bind, but $3 N-\Xi$ bound states are predicted depending on the spin-isospin two-body model dependence.

If the interaction of $\Xi$ hyperons with nuclei is sufficiently attractive to cause binding as has been repeatedly argued since the original work of (Dover and Gal, 1983), then a rich source of spectroscopic information would become available and the properties of the in-medium $\Xi N$ interaction could be extracted. Few-body cluster model calculations using the ESC04d model have been reported recently (Hiyama et al. , 2008). Bound states of $\Xi$ hypernuclei would also be useful as a gateway to form double$\Lambda$ hypernuclei (Dover, Gal, and Millener, 1994; Ikeda et al., 1994; Millener, Dover, and Gal, 1994; Yamamoto

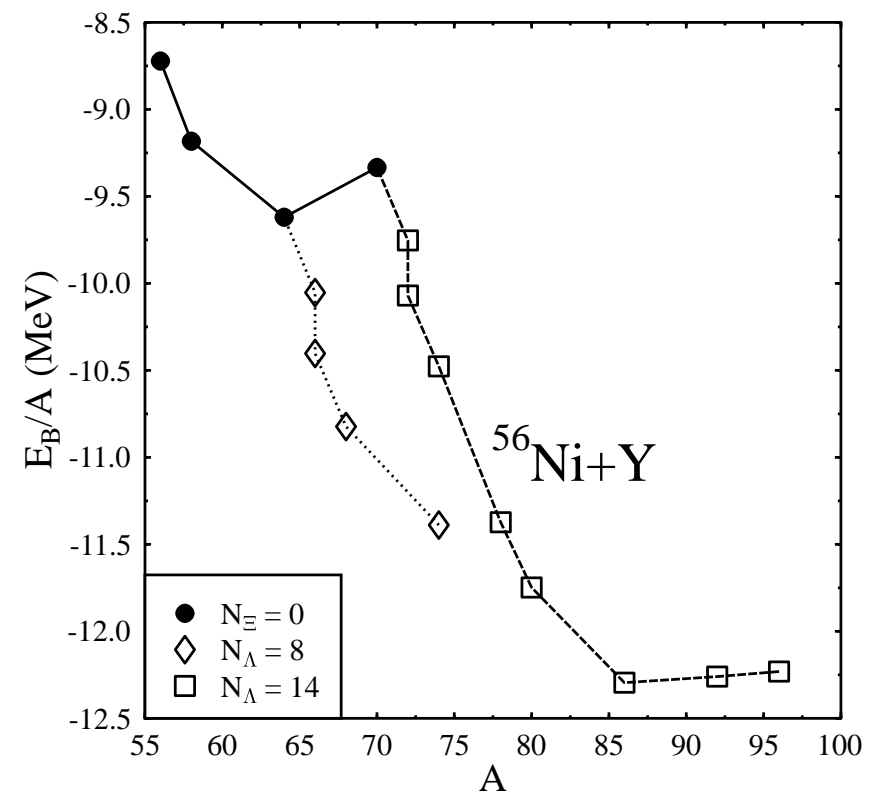

FIG. 28 Calculated binding energy of multi-strange nuclei of ${ }^{56} \mathrm{Ni}$ plus $\Lambda$ and $\Xi$ hyperons, as function of baryon number $A$. From Schaffner et al., 1993.

et al., 1994). Finally, a minimum strength of about 15 $\mathrm{MeV}$ for $-V_{0}^{\Xi}$ is required to realize the exciting possibility of "strange hadronic matter" (Schaffner-Bielich and Gal, 2000), where protons, neutrons, $\Lambda$ 's and $\Xi$ 's are held together to form a system which is stable against stronginteraction decay.

\section{STRANGE DENSE MATTER}

\section{A. Strange hadronic matter}

Bodmer (1971), and more specifically Witten (1984), suggested that strange quark matter, with roughly equal composition of $u, d$ and $s$ quarks, might provide an absolutely stable form of matter. Metastable strange quark matter was studied by Chin and Kerman (1979). Jaffe and collaborators (Farhi and Jaffe, 1984; Berger and Jaffe, 1987) subsequently charted the various scenarios possible for the stability of strange quark matter, from absolute stability down to metastability due to weak decays. Finite strange quark systems, so called strangelets, have also been considered (Farhi and Jaffe, 1984; Gilson and Jaffe, 1993).

Less known is the suggestion (Schaffner et al., 1993 , 1994 ) that metastable strange systems with similar properties, i.e., a strangeness fraction $f_{S} \equiv-S / A \approx 1$ and a charge fraction $f_{Q} \equiv Z / A \approx 0$, might also exist in hadronic form at moderate values of density, between 2 and 3 times nuclear matter density. These strange systems are made of $N, \Lambda$ and $\Xi$ baryons. The metastability (i.e., stability with respect to strong interactions, but 


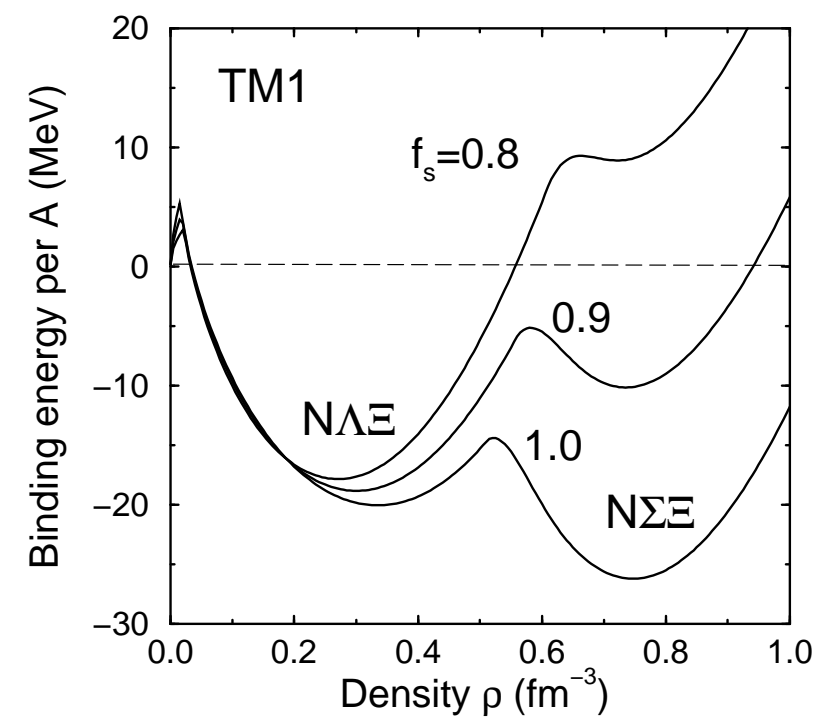

FIG. 29 Transition from $N \Lambda \Xi$ to $N \Sigma \Xi$ matter upon increasing the strangeness fraction $f_{S}$. From Schaffner-Bielich and Gal, 2000.

not to $\Delta S \neq 0$ weak-interaction decays) of these strange hadronic systems was established by extending relativistic mean field (RMF) calculations from ordinary nuclei $\left(f_{S}=0\right)$ to multi-strange nuclei with $f_{S} \neq 0$. Although the detailed pattern of metastability, as well as the actual values of the binding energy, depend specifically on the partly unknown hyperon potentials in dense matter, the predicted phenomenon of metastability turned out to be robust in these calculations (Balberg, Gal, and Schaffner, 1994). A conservative example is given in Fig. 28, assuming a relatively weakly attractive hyperon-hyperon interaction. The figure shows the calculated binding energy of ${ }^{56} \mathrm{Ni}+N_{\Lambda} \Lambda$ multi- $\Lambda$ hypernuclei for $N_{\Lambda}=0,2,8,14$ and how it becomes energetically favorable to add $\Xi$ hyperons when $N_{\Lambda}$ exceeds some fairly small threshold value. As soon as the $\Lambda p$-shell is filled, $\Xi$ hyperons may be placed in their $s$-shell owing to Pauli blocking of the stronginteraction conversion process $\Xi N \rightarrow \Lambda \Lambda$ which in free space releases about $25 \mathrm{MeV}$.

A less conservative example is provided by applying the Nijmegen soft-core model NSC97 (Stoks and Rijken, 1999) which predicts strongly attractive $\Xi \Xi, \Sigma \Sigma$ and $\Sigma \Xi$ interactions, but fairly weak $\Lambda \Lambda$ and $N \Xi$ interactions that roughly agree with existing phenomenology. Schaffner-Bielich and Gal (2000) found that strange hadronic matter (SHM) is comfortably metastable for any allowed value of $f_{S}>0$. However for $f_{S} \geq 1$, $\Sigma$ 's replace $\Lambda$ 's due to the exceptionally strong $\Sigma \Sigma$ and $\Sigma \Xi$ interactions in this model. A first-order phase transition occurs from $N \Lambda \Xi$ dominated matter for $f_{S} \leq 1$ to $N \Sigma \Xi$ dominated matter for $f_{S} \geq 1$, as shown in Fig. 29 where the binding energy is drawn versus the baryon density for several representative fixed values of $f_{S}$. At $f_{S} \approx 1.0$

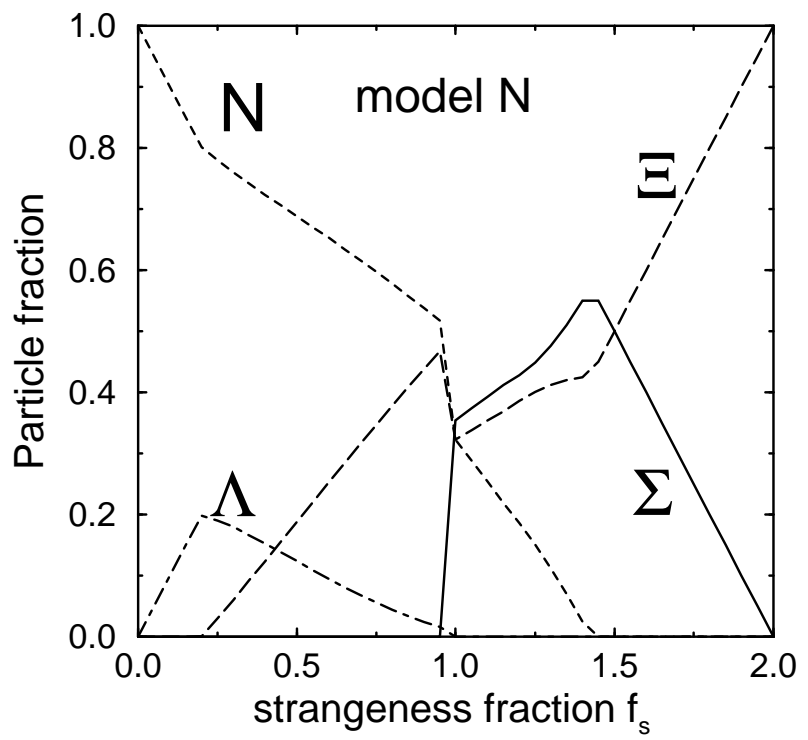

FIG. 30 Strange hadronic matter composition as function of strangeness fraction $f_{S}$. From Schaffner-Bielich and Gal, 2000 .

a secondary minimum at higher baryon density becomes energetically favored. The system then undergoes a firstorder phase transition from the low-density state to the high-density state.

Figure 30 demonstrates explicitly that the phase transition involves transformation from $N \Lambda \Xi$ dominated matter to $N \Sigma \Xi$ dominated matter, by showing the calculated composition of SHM for this model (denoted $N$ for Nijmegen) as function of the strangeness fraction $f_{S}$. The particle fractions for each baryon species change as function of $f_{S}$. At $f_{S}=0$, one has pure nuclear matter, whereas at $f_{S}=2$ one has pure $\Xi$ matter. In between, matter is composed of baryons as dictated by chemical equilibrium. A change in the particle fraction may occur quite drastically when new particles appear, or existing ones disappear. A sudden change in the composition is seen in Fig. 30 for $f_{S}=0.2$ when $\Xi$ 's (long-dashed line) emerge in the medium, or at $f_{S}=1.45$ when nucleons (short-dashed line) disappear. The situation at $f_{S}=0.95$ is a special one, as $\Sigma$ 's (solid line) appear in the medium, marking the first-order phase transition observed in the previous figure. The baryon composition alters completely at that point, from $N \Xi$ baryons plus a rapidly vanishing fraction of $\Lambda$ 's (dot-dashed line) into $\Sigma \Xi$ hyperons plus a decreasing fraction of nucleons. At the very deep minimum of the binding-energy curve (not shown here) SHM is composed mainly of $\Sigma$ 's and $\Xi$ 's with a very small admixture of nucleons. The phase transition demonstrated above has been discussed by the Frankfurt group (Schaffner et al. 2002 ) in the context of a phase transition to hyperon matter in neutron stars. Unfortunately, it will be difficult to devise an experiment to determine the depth of the $\Lambda \Xi, \Xi \Xi, \Xi \Sigma, \Sigma \Sigma$ interaction 


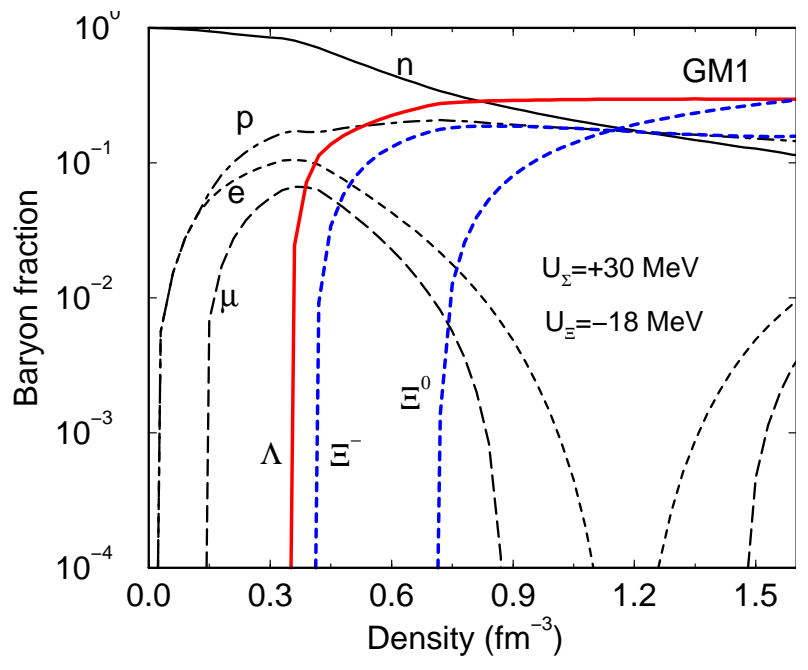

FIG. 31 Neutron star matter fractions of baryons and leptons, calculated as a function of density. From Schaffner-Bielich, 2008.

potentials, which are so crucial to verify these results.

\section{B. Neutron stars}

Neutron stars are gravitationally bound massive objects in $\beta$ equilibrium with radii of about $12 \mathrm{~km}$ and masses of about $(1-2) M_{\odot}$, perhaps up to $2.5 M_{\odot}$. Here $M_{\odot}$ stands for a solar mass (Leahy et al. 2011$)$. Although their composition at low density is dominated by neutrons, transmutation to hyperons, beginning at 2 to 3 times normal nuclear matter density $\rho_{0}=0.17 \mathrm{fm}^{-3}$, would act to alleviate the Pauli pressure of nucleons and leptons. Matter in the core of neutron stars is further compressed to about $(5-6) \rho_{0}$. At these high densities strange hadronic matter, which may already be self bound at densities $(2-3) \rho_{0}$, could become stable even to weak decay (Schaffner et al., 2002). Such matter may perhaps form kaon condensates (Kaplan and Nelson, 1986) and even deconfine to quarks (Baym and Chin, 1976), forming strange quark matter. However, it is also possible that a star having a mixed phase of hyperons and quarks in its interior is produced. Because the star rapidly rotates, losing energy via radiation, the rotational inertia of the star changes, and the rotational frequency depends on its composition which is coupled to the rotational frequency. Obviously, while more astrophysical observations are needed, the only terrestrial handle on this physics comes from hypernuclei, particularly multistrange hypernuclei. The physics of neutron stars was reviewed recently by Lattimer (2012).

It is important to recognize that hypernuclei, and, in particular, multistrange hypernuclei which were reviewed in Sec. VI.A are a low-density manifestation of strange hadronic matter. As such, studies of their interactions at

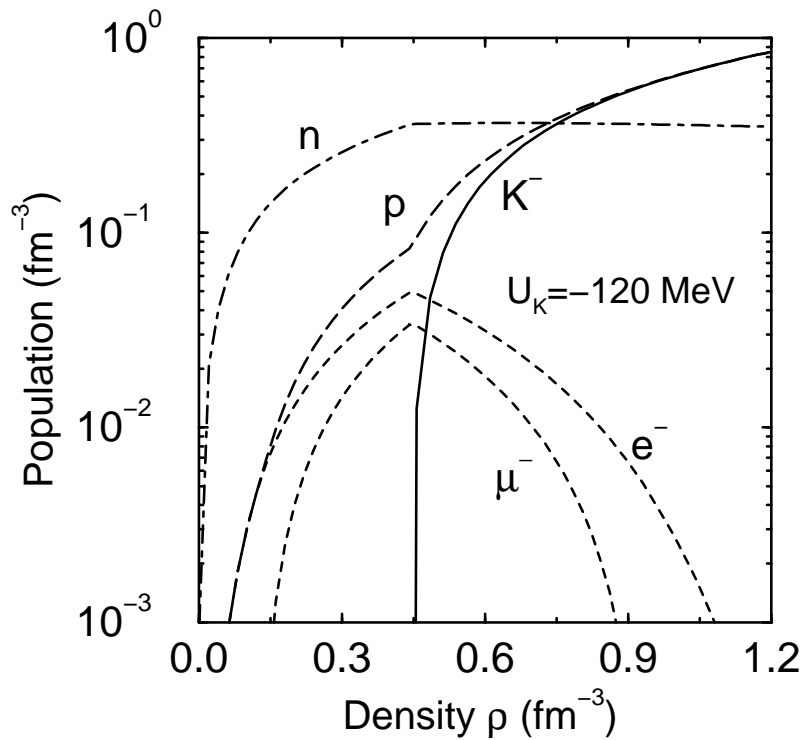

FIG. 32 Population of neutron star matter, allowing for kaon condensation, calculated as a function of nucleon density. From Glendenning and Schaffner-Bielich, 1999.

normal nuclear density impact the construction of models of density-dependent interactions for use at higher densities. Thus, hyperon potentials in dense matter control the composition of dense neutron-star matter, as shown by a recent RMF calculation in Fig. 31. As a function of density, the first hyperon to appear is the lightest one, the $\Lambda$ at about $2 \rho_{0}$, by converting protons and electrons directly to $\Lambda$ 's instead of neutrons, thereby decreasing the neutron Pauli pressure. It is reasonable to assume that this composition varies radially, perhaps having a crust and an atmosphere composed of neutrons. Among the negatively charged hyperons, the lightest one $\Sigma^{-}$does not appear at all over the wide range of densities shown owing to its repulsion in nuclear matter, and most likely also in neutron matter (Balberg and Gal, 1997). Its potential role in reducing the Pauli pressure of the leptons $\left(e^{-}\right.$and $\left.\mu^{-}\right)$could be replaced by the heavier $\Xi^{-}$hyperon, assuming overall $\Xi$-nuclear attraction. The specific calculation sketched by Fig. 31 predicts that the hyperon population overtakes the nucleon population for densities larger than about $6 \rho_{0}$, where the inner core of a neutron star may be viewed as a giant hypernucleus (Glendenning, 1985).

Negative strangeness may also be injected into neutron-star matter by agents other than hyperons. Thus, a robust consequence of the sizable $\bar{K}$-nucleus attraction, as discussed in Sec. VII, is that $K^{-}$condensation is expected to occur in neutron stars at a density about $3 \rho_{0}$ in the absence of hyperons, as shown in Fig. 32 for a RMF calculation using a strongly attractive $K^{-}$ nuclear potential $U_{\bar{K}}\left(\rho_{0}\right)=-120 \mathrm{MeV}$. Since it is more favorable to produce kaons in association with protons, the neutron density shown in the figure stays nearly con- 


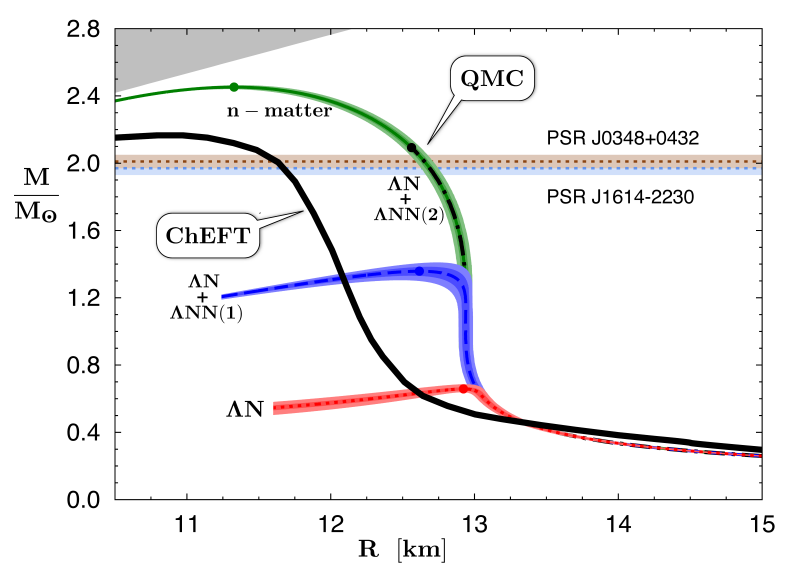

FIG. 33 Mass-radius relationship for various EoS scenarios of neutron stars, including nucleons and leptons only (Hell and Weise 2014) as well as upon including $\Lambda$ hyperons (Lonardoni et al. 2015). From Weise, 2015.

stant once kaons start to condense, while the lepton populations decrease as the $K^{-}$provides a new neutralizing agent via the weak processes $\ell^{-} \rightarrow K^{-}+\nu_{\ell}$. However, including negatively charged hyperons in the equation of state (EoS) of neutron-star matter defers $K^{-}$condensation to higher densities (Glendenning, 2001; Knorren, Prakash, and Ellis, 1995) where the neutron-star maximum mass $M_{\max }$ is lowered by only $\approx 0.01 M_{\odot}$ below the value reached through the inclusion of hyperons (Knorren, Prakhash, and Ellis, 1995).

Given the high matter density expected in a neutron star, a phase transition from ordinary nuclear matter to some exotic mixtures cannot be ruled out. Whether a stable neutron star is composed dominantly of hyperons, quarks, or some mixture thereof, and just how this occurs, is not clear as both the strong and weak interactions, which operate on inherently different time scales, are in play. The EoS of any possible composition constrains the mass-radius relationship for a rotating neutron star. Thus, the maximum mass $M_{\max }$ for a relativistic free neutron gas is given by $M_{\max } \approx 0.7 M_{\odot}$ (Oppenheimer and Volkoff, 1939: Tolman, 1939), whereas higher mass limits are obtained under more realistic EoS assumptions. Without strangeness, but for interacting nucleons (plus leptons) $M_{\max }$ comes out invariably above $2 M_{\odot}$, as shown by the curves marked $n$ matter from Quantum Monte Carlo (QMC) calculations (Lonardoni et al. 2015) and chiral effective field theory ( $\chi$ EFT) (Hell and Weise, 2014) in Fig. $33 . M_{\max }$ values of up to $2 M_{\odot}$ are within the reach of hybrid (nuclear plus quark matter) star calculations in which strangeness materializes via non-hadronic degrees of freedom (Alford et al. 2005). In the hadronic basis, adding hyperons softens the EoS, thereby lowering $M_{\max }$ in RMF calculations to the range (1.4-1.8) $M_{\odot}$ (Knorren, Prakhash, and Ellis, 1995; Glen-

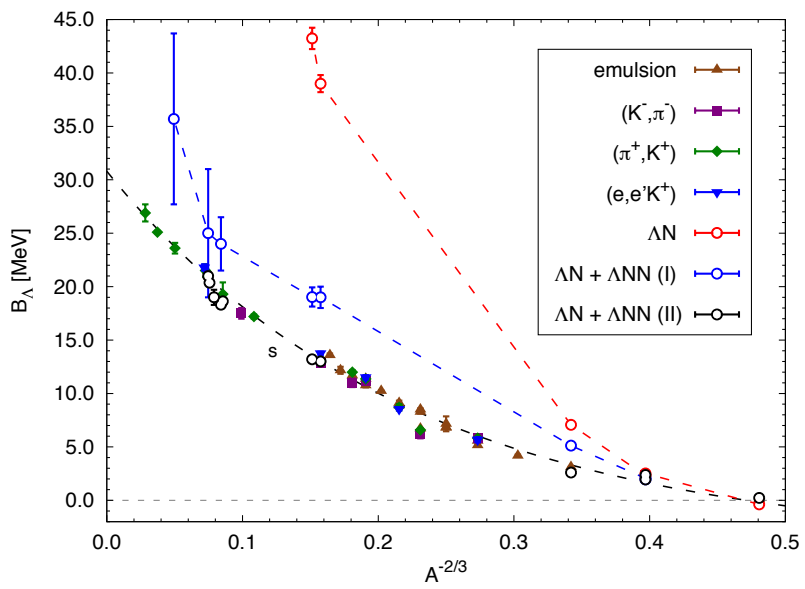

FIG. 34 QMC calculations of $\Lambda$ hypernuclear binding energies for purely two-body $\Lambda N$ interactions and for two versions of adding repulsive $\Lambda N N$ interactions. Adapted from Gandolfi and Lonardoni, 2015.

denning, 2001), also if and when a phase transition occurs to SHM (Schaffner et al. 2002). More recent HartreeFock and Bruckner-Hartree-Fock calculations using the NSC97, ESC08 and $\chi \mathrm{EFT} Y N$ interactions find values of $M_{\max }$ lower than $1.4 M_{\odot}$ (Schulze et al., 2006; Djapo, Schaefer, and Wambach, 2010; Schulze and Rijken, 2011), while the inclusion of several of the $Y Y$ interactions from the Nijmegen ESC08 model appears to increase $M_{\max }$ by $0.3 M_{\odot}$ to about $1.65 M_{\odot}$ (Rijken and Schulze, 2016).

Until recently, the neutron-star mass distribution for radio binary pulsars was given by a narrow Gaussian with mean and width values $(1.35 \pm 0.04) M_{\odot}$ (Thorsett and Chakrabarty, 1999), somewhat below the Chandrasekhar limit of $1.4 M_{\odot}$ for white dwarfs, above which these objects become gravitationally unstable. However, there is now some good evidence from x-ray binaries classified as neutron stars for masses about and greater than $2 M_{\odot}$ (Barret, Olive, and Miller, 2006). The highest $a c$ cepted value of neutron-star mass is provided at present by the precise mass measurements of the pulsars PSR J1614-2230 (Demorest et al. 2010) and PSR J0348+0432 (Antoniadis et al., 2013), marked by horizontal lines in Fig. 33 These yield nearly $2 M_{\odot}$ and thereby exclude several "soft" EoS scenarios for dense matter (Freire et al. 2009 Lattimer, 2012). The figure demonstrates how the gradual introduction of repulsive $\Lambda N N$ interactions (Lonardoni et al. 2015), from version 1 to version 2, leads to a corresponding increase of the calculated $M_{\max }$ value by increasing the matter density $\rho_{\min }$ at which $\Lambda$ hyperons appear first in neutron-star matter to higher values, until this $\rho_{\min }$ exceeds the value $\rho_{\max }$ corresponding to $M_{\max }$. When this happens, for version 2 , the massradius dotted curve overlaps with the purely " $n$-matter" green curve below the point marked in the figure for the value of $M_{\max }$ reached. This scenario in which hyperons are excluded from the EoS of neutron stars exclusively 

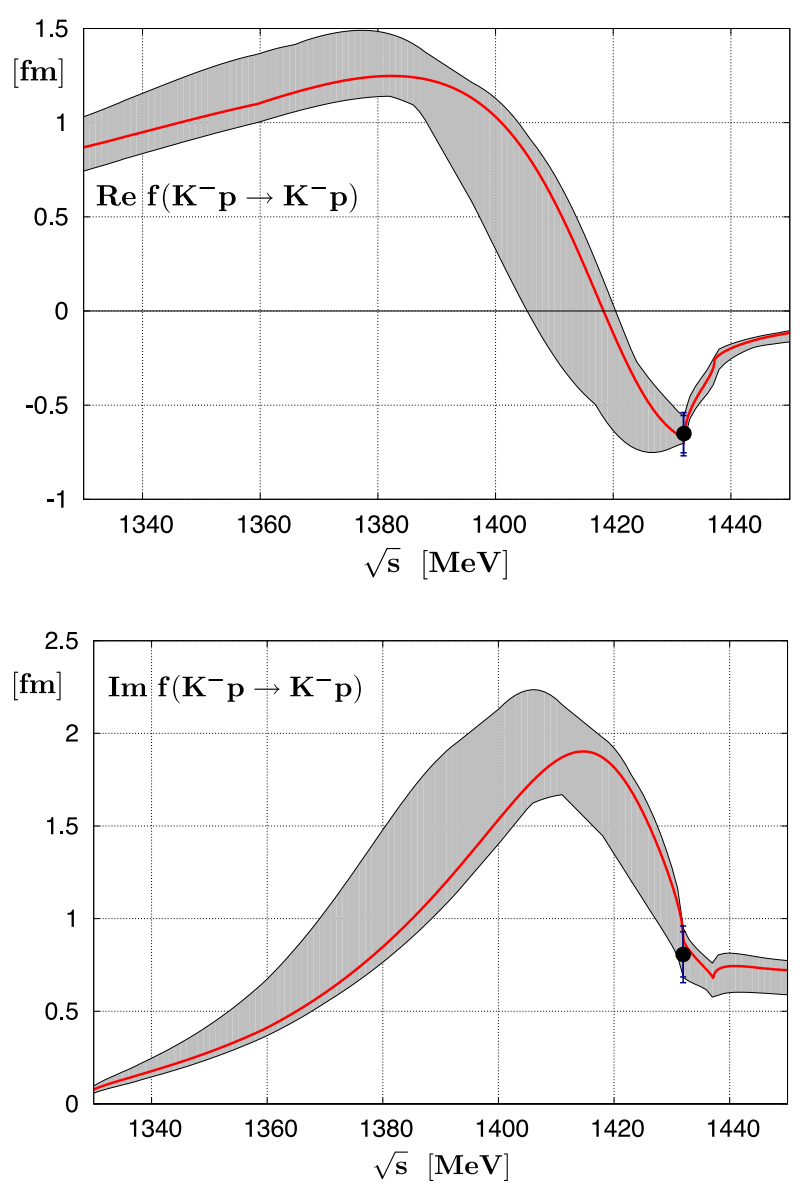

FIG. 35 NLO chiral-model calculation of the real and imaginary parts of the $K^{-} p \mathrm{~cm}$ scattering amplitude, denoted IHW in the text (Ikeda, Hyodo, and Weise, 2012). The pole position of the $\Lambda(1405)$ resonance is at $1424-i 26 \mathrm{MeV}$. The $K^{-} p$ threshold values marked by solid dots follow from the SIDDHARTA measurement of kaonic hydrogen $1 s$ level shift and width (Bazzi et al. 2011, 2012). Adapted from Ikeda, Hyodo, and Weise, 2012.

by strongly repulsive $Y N N$ forces, thereby resolving the "hyperon puzzle", requires further study.

In this context, Fig. 34 shows how the introduction of repulsive $\Lambda N N$ interactions within QMC calculations relieves the over-binding of $\Lambda$ hypernuclei which arises progressively with increasing the mass number $A$ (corresponding to smaller values of $A^{-2 / 3}$ in the figure) upon using microsocopically constructed purely two-body $\Lambda N$ interactions dominated by attraction. In particular, the same version " $\Lambda N+\Lambda N N$ (II)" that according to Fig. 33 resolves the hyperon puzzle also resolves, according to Fig. 34, the " $B_{\Lambda}$ over-binding" problem. It is worth noting, however, that the purely two-body $\Lambda N$ interaction of version ' $\Lambda N$ ' overbinds heavy $\Lambda$ hypernuclei substantially beyond the $\Lambda N$ two-body contribution $D_{\Lambda}^{(2)} \sim 60 \mathrm{MeV}$ to the $\Lambda$-nucleus potential well depth derived from the $A$ dependence of the $\left(\pi^{+}, K^{+}\right)$-measured $\Lambda$ binding en- ergies (Millener, Dover, and Gal, 1988). This excessive overbinding is then compensated in Lonardoni, Pederiva, and Gandolfi (2014) by a similarly excessive $\Lambda N N$ repulsion which makes the neutron-star matter EoS so stiff as to exclude hyperons from appearing in neutron-star matter. In other, phenomenological models that introduce softer repulsive $\Lambda N N$ interactions in a more controlled way, values of $M_{\max }$ in the range $(1.6-1.7) M_{\odot}$ are obtained (Balberg and Gal, 1997, Vidaña et al., 2011), short however of resolving the hyperon puzzle. Nevertheless, it is possible to reach values of $M_{\max } \geq 2 M_{\odot}$ by introducing in addition to moderately repulsive $\Lambda N N$ interactions also phenomenological repulsive $N N N$ interactions that have not been tested yet in nuclear structure calculations (Yamamoto et al., 2013, 2014, 2016). Obviously, more work is required in this direction to make sure whether or not the hyperon puzzle is indeed resolved; see Chatterjee and Vidaña (2016) for a comprehensive review of related works.

\section{VII. $\bar{K}$-NUCLEAR INTERACTIONS AND BOUND STATES}

The $\bar{K} N$ interaction near and below threshold is attractive in models which dynamically generate the $\Lambda$ (1405) subthreshold resonance. This motivates a search for $K^{-}$quasibound states in nuclei (Gal, 2013; Hyodo, 2013). The $\Lambda(1405)$ was predicted as early as 1959 (Dalitz and Tuan, 1959) by analyzing the available data on the strong interactions of $K^{-}$mesons with protons above threshold, and was discovered two years later in the Berkeley hydrogen bubble chamber Alston et al., 1961) as an $I=0 \pi \Sigma$ resonance by studying the reaction $K^{-} p \rightarrow \Sigma+3 \pi$ for several charge states. The proximity of this $\pi \Sigma$ resonance to the $\bar{K} N$ threshold, at 1432 $\mathrm{MeV}$ for $K^{-} p$, suggested that it can be dynamically generated by $\bar{K} N-\pi \Sigma$ interhadron forces. This was subsequently shown (Dalitz, Wong, and Rajasekaran, 1967) to be possible within a dynamical model of SU(3)-octet vector-meson exchange. The model provides a concrete physical mechanism for the Tomozawa-Weinberg leading term in the chiral expansion of the meson-baryon Lagrangian (Tomozawa, 1966; Weinberg, 1966).

A NLO chiral-model calculation of the $K^{-} p$ centerof-mass (c.m.) scattering amplitude $f_{K^{-}}$is shown in Fig. 35. This NLO amplitude agrees qualitatively with leading-order $K^{-} p$ amplitudes derived in the mid 1990s [see, e.g., Kaiser, Siegel, and Weise (1995) and Oset and Ramos (1998)], the main quantitative improvement arising from the threshold value constraint provided by the SIDDHARTA measurement of kaonic hydrogen $1 s$ level shift and width (Bazzi et al. 2011,2012$)$. The large positive values of Re $f_{K^{-}}$, which exceed $1 \mathrm{fm}$ in the subthreshold region, indicate a strong attraction. Although all NLO models agree above threshold, because of fitting to the same $K^{-} N$ low-energy scattering and reaction 
TABLE XVII Calculated $K^{-} p p$ binding energies $B \&$ widths $\Gamma$. DHW stands for Doté, Hyodo, and Weise (2008, 2009), BGL for Barnea, Gal, and Liverts (2012), IKS for Ikeda, Kamano, and Sato (2010), RS for Révai and Shevchenko (2014), YA for Yamazaki and Akaishi (2002), WG for Wycech and Green (2009), SGM for Shevchenko, Gal and Mareš (2007) and Shevchenko et al. (2007), and IS for Ikeda and Sato (2007, 2009).

\begin{tabular}{lcccc}
\hline \hline \multicolumn{5}{c}{$\begin{array}{c}\text { Energy dependent meson-baryon interactions } \\
\text { Variational }\end{array}$} \\
$(\mathrm{MeV})$ & DHW & BGL & IKS & Faddeev \\
\hline$B$ & $17-23$ & 16 & $9-16$ & 32 \\
$\Gamma$ & $40-70$ & 41 & $34-46$ & 49 \\
\multicolumn{5}{c}{ Energy independent meson-baryon interactions } \\
$B$ VeV) & Yariational & WG & SGM & Faddeev \\
$\Gamma$ & 48 & $40-80$ & $50-70$ & IS \\
\hline \hline
\end{tabular}

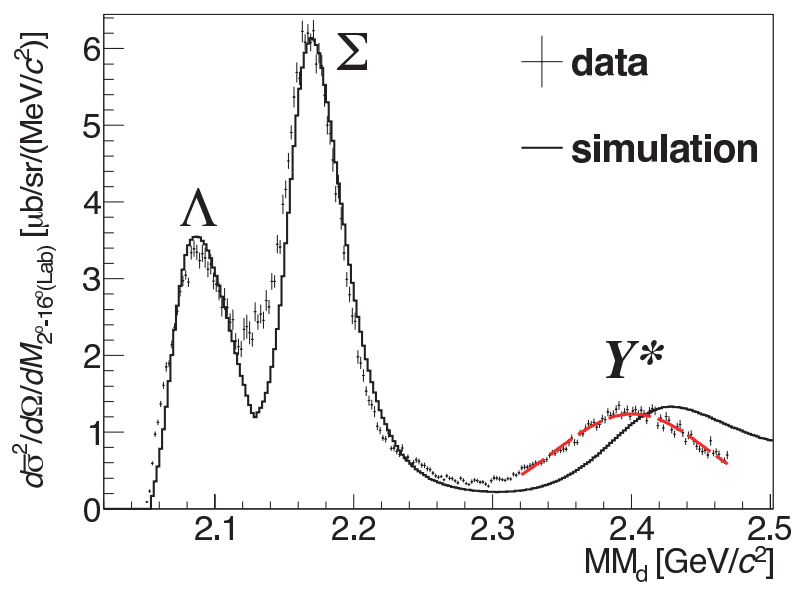

FIG. 36 Missing-mass spectrum $\left(\mathrm{MM}_{\mathrm{d}}\right)$ of the $d\left(\pi^{+}, K^{+}\right)$ reaction in the J-PARC E27 experiment at forward angles. A phase-space simulated spectrum is shown by a solid line. Adapted from Ichikawa et al., 2014.

data, a non-negligible model dependence below threshold can be deduced by comparing to other NLO chiral calculations; see, e.g., Guo and Oller (2013). However, it is the subthreshold region that is needed in bound-state calculations, which is also true for kaonic atoms where the kaon energy is essentially at threshold (Gal et al. 2014). Fortunately, the two $K^{-} N$ scattering amplitudes used in the most recent atomic and nuclear quasi-boundstate calculations, IHW (Ikeda, Hyodo, and Weise, 2011, 2012) of Fig. 35 and NLO30 (Cieplý and Smejkal, 2012) shown in a later figure, are also similar in the subthreshold region despite the different methodologies involved in their derivations.

The lightest $\bar{K}$-nuclear quasibound state is expected to be $K^{-} p p$. Such a $\bar{K} N N$ state would have isospin $I=\frac{1}{2}$ and spin-parity $J^{\pi}=0^{-}$, dominated by $I_{N N}=1$ and $s$ waves. A representative compilation of recent few-body

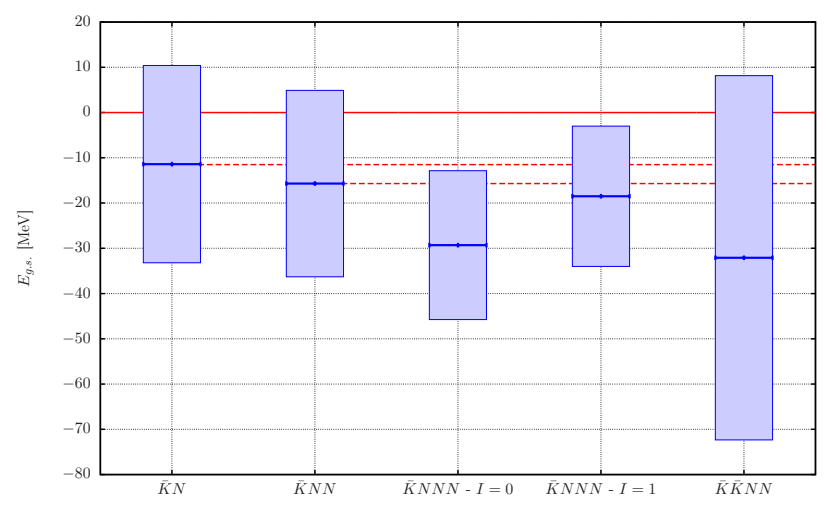

FIG. 37 Binding energies and widths, $\Gamma(\bar{K} N \rightarrow \pi Y)$, of $\bar{K}$ and $\bar{K} \bar{K}$ few-body quasibound states (in $\mathrm{MeV}$ ) calculated by Barnea, Gal, and Liverts (2012). Horizontal lines denote particle-stability thresholds. Widths are represented by vertical bars. A possible $I=\frac{1}{2}, J^{\pi}=\frac{1}{2}^{+} \bar{K} \bar{K} N$ quasibound state (Shevchenko and Haidenbauer, 2015) is not shown in this figure. Figure courtesy of N. Barnea.

calculations of this system is given in Table XVII. These calculations suggest robust binding for $K^{-} p p$, but the calculated widths are all large (of order $50 \mathrm{MeV}$ ). The table shows that chiral-model calculations using energydependent $\bar{K} N$ interactions give weaker binding than those calculated when disregarding the energy dependence away from the $\bar{K} N$ threshold. Since the $K^{-} p p$ quasi-bound state may be regarded as $\Lambda(1405) N$ bound state (Uchino, Hyodo, and Oka, 2011), this difference partly reflects the higher $\Lambda(1405)$ mass obtained in chiral models [see the caption to Fig. 35 for the $\Lambda(1405)$ pole position in that calculation].

While several experiments have suggested evidence for a $K^{-} p p$ quasibound state with somewhat conflicting binding energy, there seems to be no consensus on this matter and it awaits further experimentation. In Fig. 36, a missing-mass spectrum is shown for the $d\left(\pi^{+}, K^{+}\right)$reaction at $1.69 \mathrm{GeV} / \mathrm{c}$ taken at J-PARC (Ichikawa et al. 2014). The main features of this spectrum are the quasifree $\Lambda, \Sigma$ and $Y^{*}$ components. The latter rests on a broad phase-space structure. As for dynamical structures aside from the expected $\Sigma N$ cusp structure around 2.13 $\mathrm{GeV} / \mathrm{c}^{2}$, one observes a $20-30 \mathrm{MeV}$ downward shift of the broad bump representing the $Y^{*}$ component. This indicates attraction for the $Y^{*} N$ system. Unfortunately, in this kinematical region the contributions of $\Sigma(1385)$ and $\Lambda(1405)$ overlap and are indistinguishable. A $\Sigma(1385) N$ quasibound realization of such a structure was previously discussed by Gal and Garcilazo (2013) as a possible $I=\frac{3}{2}, J^{\pi}=2^{+} \pi Y N$ resonance near the $\pi \Sigma N$ threshold (about $100 \mathrm{MeV}$ below the $\bar{K} N N$ threshold). The main attraction in this "pion-assisted dibaryon" comes from the $p_{3 / 2}$-wave pion-baryon interactions, where $\bar{K} N N$ admixtures play a negligible role. 
Of the $K^{-} p p$ calculations listed in Table XVII we chose to review the hyperspherical-basis variational calculations including also four-body bound states (Barnea, Gal, and Liverts, 2012). The energy dependence of the $\bar{K} N$ interaction in this calculation is treated self consistently. The binding energies are shown in Fig. 37 for three- and four-body kaonic bound states. $\Gamma(\bar{K} N \rightarrow \pi Y)$ width estimates are plotted as vertical bars, given by

$$
\frac{\Gamma}{2} \approx\left\langle\Psi_{\text {g.s. }}\left|-\operatorname{Im} \mathcal{V}_{\bar{K} N}\right| \Psi_{\text {g.s. }}\right\rangle,
$$

where $\mathcal{V}_{\bar{K} N}$ consists of all pairwise $\bar{K} N$ interactions. Equation (53) provides a good approximation because $\left|\operatorname{Im} \mathcal{V}_{\bar{K} N}\right| \ll\left|\operatorname{Re} \mathcal{V}_{\bar{K} N}\right|$ (Hyodo and Weise, 2008). The calculated binding energies (widths) typically are found to be 10 (10 to 40) MeV lower than when one uses threshold values as input, due to the self-consistency requirement which results in weaker $\bar{K} N$ interactions below threshold. In particular, the $I=\frac{1}{2} \bar{K} N N$ g.s. $\left(K^{-} p p\right)$ lies only $4.3 \mathrm{MeV}$ below the $11.4 \mathrm{MeV}$ centroid of the $I=0 \bar{K} N$ quasibound state. The latter value differs substantially from the $27 \mathrm{MeV}$ binding energy traditionally assigned to the $\Lambda(1405)$ resonance used in nonchiral calculations. The $\bar{K} N \rightarrow \pi Y$ widths are of order 40 $\mathrm{MeV}$ for single- $\bar{K}$ clusters and twice that for double- $\bar{K}$ clusters. Additional $\bar{K} N N \rightarrow Y N$ contributions of up to $\sim 10 \mathrm{MeV}$ in $K^{-} p p$ (Doté, Hyodo, and Weise, 2009) and $\sim 20 \mathrm{MeV}$ in the four-body systems (Barnea, Gal, and Liverts, 2012) are likely.

For calculations involving heavier single- $\bar{K}$ nuclear systems one needs in-medium $\bar{K} N$ scattering amplitudes. The in-medium $K^{-} N$ isoscalar amplitudes obtained from the chirally motivated coupled-channel model of Cieplý and Smejkal (2012), and denoted NLO30 in the text, are shown in Fig. 38 above and below threshold. The real part of the subthreshold amplitude, which is relevant to $K^{-}$atomic and nuclear states, is strongly attractive $(\sim 1 \mathrm{fm})$ and similar to that of the IHW subthreshold amplitude. This implies that $K^{-}$quasibound states are likely to exist. Note that the attraction as well as absorption (expressed by the imaginary part of the amplitude) become moderately weaker for $\rho \geq 0.5 \rho_{0}$, as demonstrated by comparing the solid $\left(\rho=\rho_{0}\right)$ and dashed curves $\left(\rho=0.5 \rho_{0}\right)$.

The NLO30 in-medium $\bar{K} N s$-wave scattering amplitudes shown in Fig. 38 were used by Gazda and Mareš (2012) to evaluate self-consistently $K^{-}$quasibound states using RMF nuclear-core densities across the periodic table. Calculated $K^{-}$binding energies, $B_{K}$, and widths, $\Gamma_{K}$, in Ca are listed in Table XVIII for several choices of input interactions. Listed in the table are also values of $B_{K}$ and $\Gamma_{K}$ derived by adding a $\Sigma(1385)$-motivated $p$-wave $K^{-} N$ interaction from Weise and Härtle (2008). This marginally increases $B_{K}$ by a few $\mathrm{MeV}$ and modifies $\Gamma_{K}$ by less than $1 \mathrm{MeV}$. By adding a two-nucleon $(2 N)$ $K^{-} N N \rightarrow Y N$ absorption term estimated from fitting to
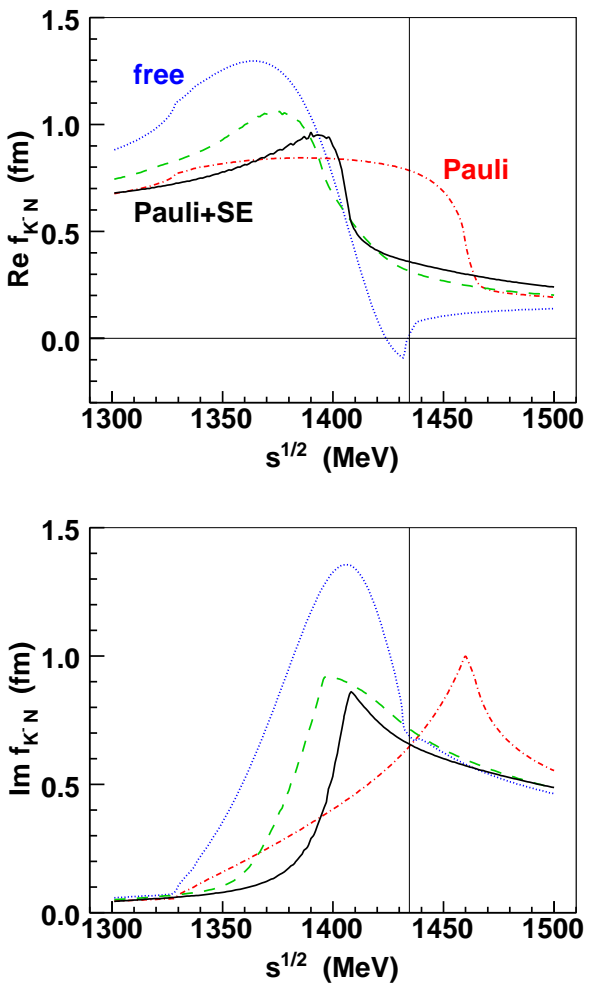

FIG. 38 Near-threshold energy dependence of $K^{-} N$ centerof-mass scattering amplitudes in model NLO30 Cieplý and Smejkal, 2012) for free-space (dotted) and Pauli-blocked amplitudes at $\rho=\rho_{0}$ with (solid) and without (dot-dashed) meson and baryon self-energies (SE). The dashed curves show Pauli-blocked amplitudes with SE at $\rho=0.5 \rho_{0}$. The $K^{-} N$ threshold is marked by a thin vertical line. Figure courtesy of A. Cieplý.

kaonic atoms, a $\lesssim 2 \mathrm{MeV}$ decrease of $B_{K}$ results, but the width substantially increases to $\Gamma_{K} \sim(50-70) \mathrm{MeV}$. Given these large widths, it is unlikely that distinct quasibound states can be uniquely resolved, except perhaps in very light $K^{-}$nuclei.

The hierarchy of widths listed in Table XVIII is also worth noting. One expects a maximal width in the lowest, most localized $1 s_{K}$ states for energy-independent po-

TABLE XVIII Self-consistently calculated Gazda and Mareš 2012) binding energies $B_{K}$ and widths $\Gamma_{K}$ (in $\mathrm{MeV}$ ) of $K^{-}$quasi-bound states in Ca using a static RMF Ca density and NLO30 in-medium $K^{-} N$ subthreshold amplitudes (Cieplý and Smejkal, 2012.

\begin{tabular}{lcccccc}
\hline \hline & \multicolumn{2}{c}{ NLO30 } & \multicolumn{2}{c}{$+p$ wave } & \multicolumn{2}{c}{$+2 N$ abs. } \\
& $B_{K}$ & $\Gamma_{K}$ & $B_{K}$ & $\Gamma_{K}$ & $B_{K}$ & $\Gamma_{K}$ \\
\hline $1 s_{K}$ & 70.5 & 14.9 & 73.0 & 14.8 & 68.9 & 58.9 \\
$1 p_{K}$ & 50.6 & 18.0 & 53.1 & 17.9 & 49.2 & 53.6 \\
$1 d_{K}$ & 28.8 & 30.3 & 32.1 & 29.3 & 27.7 & 59.7 \\
$2 s_{K}$ & 23.9 & 33.8 & 26.3 & 34.2 & 21.6 & 67.1 \\
\hline \hline
\end{tabular}




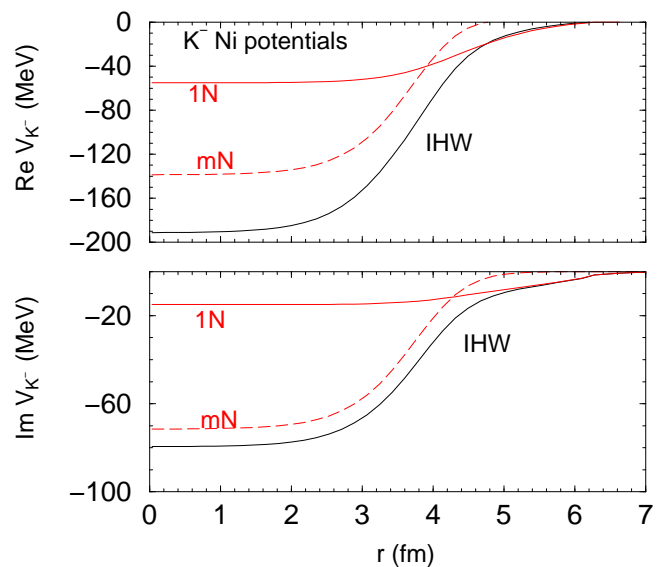

FIG. 39 A self-consistent $K^{-}$nuclear potential $V_{K^{-}}$for $K^{-}$ atoms of Ni derived from global fits (Friedman and Gal, 2013) based on in-medium IHW one-nucleon $(1 N)$ amplitudes, together with its $1 N$ and multinucleon $(m N)$ components.

tentials, which gradually decreases in excited states since these are less localized within the nucleus. The reverse is observed here, particularly when excluding $2 N$ absorption. This is a corollary of the required self consistency; the more excited a $K^{-}$quasibound state, the lower nuclear density it feels and thus a smaller subthreshold downward shift it experiences. Since $\operatorname{Im} f_{K^{-} N}(\rho)$ decreases strongly below threshold (see Fig. 38) the contribution to the calculated width gets larger as the excitation energy of the quasibound state increases.

$K^{-}$nucleus optical potential fits to kaonic-atom data across the periodic table reveal that the in-medium IHWbased, or NLO30-based one-nucleon $(1 N)$ amplitude input to $V_{K^{-}}$fails to reproduce, even qualitatively, the $K^{-}$atomic level shifts and widths. This is demonstrated in Fig. 39 by the considerably stronger component, attributed to multinucleon $(m N)$ processes with $m=2,3, \cdots$, of the fitted $V_{K^{-}}$. The composition of the imaginary part of the potential is of particular interest. It indicates that the $m N$ component, which is sizable in the nuclear interior, becomes negligible about half a fermi outside the half-density radius. This has implications for optimally choosing the kaonic-atom candidates where widths of two atomic levels can be measured (Friedman and Okada, 2013) to substantiate the $1 N$ vs $m N$ pattern observed in global fits (Friedman and Gal, 2012, 2013). Finally, Fig. 40 demonstrates that both IHW and NLO30 energy-dependent in-medium amplitude inputs to $V_{K^{-}}$ lead to practically the same strongly attractive and absorptive nuclear-matter potential $V_{K^{-}}\left(\rho_{0}\right)$.

It is worth noting that the strong $K^{-}$nuclear attraction forces the atomic $K^{-}$wavefunction to overlap appreciably with the nuclear density down to almost $90 \%$ of the central nuclear density $\rho_{0}$ (Friedman and Gal, 2007; Gal, 2013). This does not hold for the shallower optical poten-

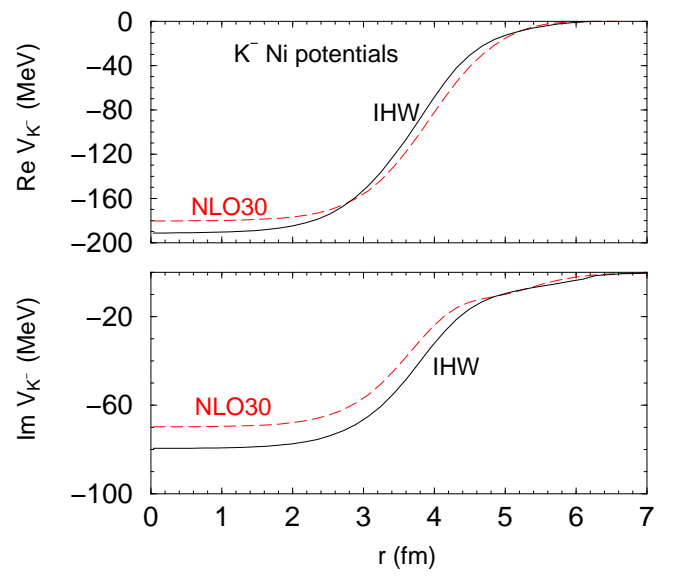

FIG. 40 Self-consistent $K^{-}$nuclear potentials $V_{K^{-}}$for $K^{-}$ atoms of Ni derived from global fits (Friedman and Gal, 2013) based on the in-medium IHW $1 N$ amplitudes (solid curves) (see Fig. 39 and as based on the in-medium NLO30 $1 \mathrm{~N}$ amplitudes (dashed curves).

tials $V_{K^{-}}$based on $1 N$ energy-independent $f_{K^{-} N}$ input consisting of threshold values (Baca, Garcia-Recio, and Nieves, 2000). Such potentials do not penetrate significantly beyond $10 \%$ of $\rho_{0}$ and also do not provide equally good atomic fits as shown in Fig. 22 of Friedman and Gal (2007). In this context, a reaction that discriminates between deep and shallow attractive $K^{-}$nuclear potentials is the formation of $\Lambda$ hypernuclear states localized within the nuclear interior in $K^{-}$capture at rest. The calculated formation rates show sensitivity to how far the relevant $K^{-}$atomic wavefunctions penetrate into the nucleus (Cieplý et al. 2011). Formation rates of several $p$-shell hypernuclear ground states, available from FINUDA experiments (Agnello et al. 2011b) and analyzed by Cieplý et al. (2011), favor deep $K^{-}$nuclear potentials to shallow ones.

One might expect increased binding in multi- $K^{-}$nuclei when calculated using strongly attractive $K^{-}$nuclear potentials, which are fitted to $K^{-}$atom data, since the bosonic nature of kaons allows them to occupy the same high-density central region of nuclei. This turns out not to be the case, as demonstrated by the RMF calculations of Gazda et al. (2008) shown in Fig. 41. The difference between the various curves representing a given starting value of $B_{K^{-}}$, originates from the balance of the RMF inputs between the vector fields which generate $\bar{K} \bar{K}$ repulsion and the $\sigma$ scalar field which generates overall attraction. The separation energies, $B_{K^{-}}$, saturate as a function of the number of $K^{-}$mesons, $\kappa$, such that $B_{K^{-}}(\kappa \rightarrow \infty) \ll\left(m_{K}+M_{N}-M_{\Lambda}\right) \approx 320 \mathrm{MeV}$. This implies that antikaons do not replace $\Lambda$ hyperons in the ground-state realization of multistrange hadronic systems. Stated differently, antikaons do not condense in a finite self-bound hadronic system. 


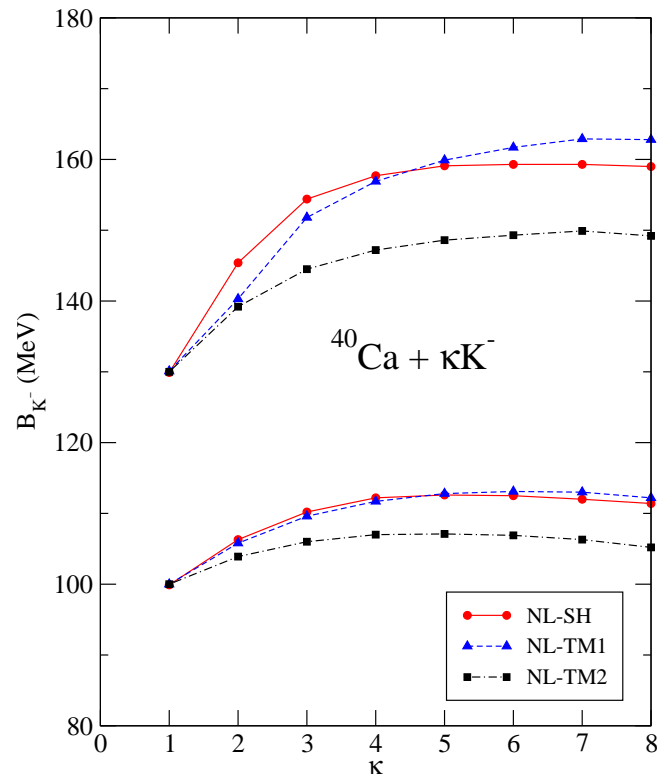

FIG. 41 Saturation of $1 s_{K^{-}}$separation energies $B_{K^{-}}$as calculated in multi- $K^{-}\left({ }^{40} \mathrm{Ca}+\kappa K^{-}\right)$nuclei (Gazda et al. 2008) for several versions of RMF input marked in the inset. The lower (upper) group of curves was constrained to produce $B_{K^{-}}=100$ (130) $\mathrm{MeV}$ for $\kappa=1$.

\section{FUTURE EXPERIMENTS AND DIRECTIONS}

Even though $\mathrm{SU}(3)_{\mathrm{f}}$ symmetry is badly broken, it is a useful way to organize the discussion of strangeness within a nucleus. Thus techniques in, and knowledge of, traditional nuclear physics may readily be applied. As examples, spectroscopy that resolves the spin structure, and the weak decay mechanisms that operate within the nuclear interior illuminate new features of the hadronic many-body problem.

Because the $\Lambda \mathrm{N}$ interaction is weak, hypernuclear spectroscopy can be represented by a superposition of particle-hole states resulting in $5-10 \mathrm{MeV}$ spaced $\hbar \omega$ structures, and these can be resolved, as previously discussed, by experiments with $1-2 \mathrm{MeV}$ resolution. However, it is more difficult to extract levels which involve nuclear-core excitations, or to resolve $\Lambda$ spin-flip excitations within the enclosing $\hbar \omega$ structures. Indeed, direct observation of $\Lambda$ spin-doublet structure in many instances requires resolutions approaching $100 \mathrm{keV}$ or better, and thus well beyond the capabilities of present magnetic spectroscopy. Still, resolution of nuclear-core excitations at the $\leq 500 \mathrm{keV}$ level carry substantial physics interest, and are accessible with modern, continuous-beam electron accelerators (Nakamura, 2013), and perhaps also with meson beams at the J-PARC (Japan Proton Accelerator Research Complex) $50 \mathrm{GeV}$ proton synchrotron (Takahashi, 2013).

In addition to spectroscopy, nonmesonic weak decays provide information on the local nuclear environment, in- cluding for example $N N$ correlations. Also by comparing energy shifts between charge-symmetric hypernuclei, information on the dynamical behavior of the nuclear core and the admixture of other hyperons in the ground-state wave function can be obtained. Finally, multihyperon states provide information on hyperon-hyperon interactions that is needed to extend $S U(3)_{\mathrm{f}}$ symmetry and develop a better understanding of nuclear matter at high density in astrophysical objects.

Future programs will be driven by the new proton accelerator at J-PARC, the continuous electron accelerators at Jlab and Mainz, and the antiproton facility at FAIR. Not only do these facilities have infrastructure designed for hypernuclear research, but the experiments will be able to take advantage of new, innovative detectors and electronics that will allow higher rates, better energy resolution, and better particle and signal identification. It is anticipated that this field will remain interesting and fertile to new exploration.

\section{A. Spectroscopy using meson beams}

\section{Hyperon production and hyperon-nucleon interactions}

As discussed in Secs. I I and II the mainstays of hypernuclear research have been the $\left(K^{-}, \pi^{-}\right)$and $\left(\pi^{+}, K^{+}\right)$ mesonic reactions. On the other hand, studies of heavy hypernuclear systems may prove difficult. Therefore, it is important to undertake better measurements of elementary hyperon production cross sections and, in particular, polarization observables may prove useful. Polarization is small at the forward angles where the $\Lambda$ production amplitude is sufficient to be experimentally useful. However, polarization is crucial in experiments attempting to measure the weak-decay asymmetry. Although the residual polarization after hypernuclear production appears consistent with zero, polarization due to the large spinflip amplitudes in the $\left(K^{-}, \pi^{-}\right)$reaction at 1.1 and 1.5 $\mathrm{GeV} / \mathrm{c}$ has not been explored systematically. This may be more accessible with the intense kaon beams available at J-PARC, as indeed proved in the E13 experiment by populating the ${ }_{\Lambda}^{4} \mathrm{He}\left(1^{+}\right)$level in the $\left(K^{-}, \pi^{-}\right)$reaction on ${ }^{4} \mathrm{He}$ at $p_{K}=1.5 \mathrm{GeV} / \mathrm{c}$ (Yamamoto et al., 2015).

Most importantly, there should be a plan to systematically study the elementary hyperon-nucleon $(Y N)$ interaction. To date only approximately 40 data points of $Y N$ scattering cross sections are available from mostly old experiments that studied hyperon post-production secondary interactions. Some of the more recent $\Sigma N$ data were obtained using the SCIFI (scintillator fiber) active detector system of the 1990s. One approved experiment at J-PARC, E40 (Takahashi, 2013), will extend these measurements. Such new and improved data are particularly important from a theoretical standpoint in constructing $Y N$ potential models for use in hypernu- 
clear structure applications. We recall from Table XIV that successive Nijmegen ESC potentials, the latest of which is ESC08 (Nagels, Rijken, and Yamamoto, 2015a, 2015b), have led to increasingly repulsive $\Sigma$-nucleus $G$ matrix potentials, in agreement with deductions made from $\Sigma$ hypernuclear production experiments. Therefore, it would be useful to enhance the $Y N$ data base of these models by new and more precise $\Sigma N$ cross section data in order to confirm the validity of these deductions. Similarly, it would be useful to enhance the $S=-2$ baryonbaryon data base by new and more precise $\Xi N$ cross section data, particularly by remeasuring and extending the poorly measured $\Xi^{-} p \rightarrow \Lambda \Lambda$ reaction cross sections. This input is crucial for confirming that the $S=-2$ baryonbaryon interactions are fairly weak, as suggested by the absence of a particle-stable $H$ dibaryon and by the accurately known $B_{\Lambda \Lambda}\left({ }_{\Lambda \Lambda}^{6} \mathrm{He}\right)$ value, and in agreement with a recent NLO $\chi$ EFT study by Haidenbauer, Meißner, and Petschauer (2016).

\section{Reaction spectroscopy with mesons}

The absence of a modern hadron accelerator, providing intense beams of energetic kaons and pions, has hindered the exploration of hypernuclear experiments, particularly those involving the study of doubly-strange nuclear systems. This impediment is being resolved with the introduction of experiments at J-PARC (Takahashi, 2013). The $30 \mathrm{GeV}$ proton beam at J-PARC is operative, producing various high-intensity beams of secondary pions and kaons. Two beamlines are initially available, with high-resolution magnetic spectrometers that are able to reach missing-mass resolution of somewhat less than $2 \mathrm{MeV}$ at best. A proposed high-resolution $\left(\pi^{+}, K^{+}\right)$spectrometer for use in a future extension of the hadron facility should achieve missing mass resolutions for hypernuclear spectroscopy of $\leq 500 \mathrm{keV}$. So far, the spectroscopy of single- $\Lambda$ hypernuclei has been addressed in brief running periods of experiments E10, search for ${ }_{\Lambda}^{6} \mathrm{H}$ (Sugimura et al. 2014$)$, and E13, $\gamma$-ray studies in the $s, p$, and $s d$ shells (Tamura et al., 2013), with the latter observing a $1.41 \mathrm{MeV}^{+} \rightarrow 0^{+} \gamma$ transition in ${ }_{\Lambda}^{4} \mathrm{He}$ (Yamamoto et al. 2015). Also high on the hypernuclear agenda is experiment E05 which is a search for the ${ }_{\Xi}^{12} \mathrm{Be}$ hypernucleus via ${ }^{12} \mathrm{C}\left(K^{-}, K^{+}\right){ }_{\Xi}^{12} \mathrm{Be}($ Nagae, 2013). In this experiment, the overall energy resolution in the $\Xi^{-}$bound-state region is expected to be in the range of $1.5-3 \mathrm{MeV}$ at FWHM.

\section{Experiments using emulsion detectors}

As described earlier, nuclear emulsion was the first detection system used to investigate hypernuclear events. The advantage of emulsion is its excellent position and energy resolution, which allows detailed investigation of a reaction and its decay products. Coupling counters with emulsion, although somewhat clumsy, can still provide needed information under certain experimental conditions. Indeed, this technique was crucial in the KEK E373 determination of the binding energy of ${ }_{\Lambda \Lambda}^{6} \mathrm{He}$ (Takahashi et al., 2001; Ahn et al., 2013). A coupled counter and emulsion detector is proposed for the study of $\Lambda \Lambda$ systems at J-PARC. In this experiment E07 $\Xi^{-}$are produced in a diamond target upstream of the emulsion and are tracked as they recoil into, and stop, in the emulsion (Takahashi, 2013). Particle emission from the stopping vertex is then analyzed for various reactions, including the production of $S=-2$ systems.

\section{Spectroscopy using electromagnetic transitions}

While the energy resolution using direct spectroscopy to specific states with magnetic spectrometers and meson beams is presently limited to no better than a few hundred $\mathrm{keV}$, the energy of electromagnetic transitions between states can be measured to a few keV. Thus, measurement of electromagnetic transitions is a powerful tool for hypernuclear spectroscopy. This requires a dedicated beam line to tag the formation of a specific hypernucleus, and large acceptance, high resolution Ge detectors. The photon detectors to be used have high photo-peak efficiency and rate handling capabilities. The system at J-PARC is called Hyperball-J (Tamura et al. 2013 ) and consists of 28 mechanically-cooled Ge detectors having $60 \%$ relative efficiency. Each Ge crystal is enclosed by $2 \mathrm{~cm}$ thick lead tungstate (PWO) counters to suppress Compton scattering and $\gamma$ rays from $\pi^{0}$ decays. The readout requires special electronics for high counting rate and large dynamic range of the signals.

J-PARC has tested and mounted equipment to undertake a study of $\gamma$ emission from excited levels in ${ }_{\Lambda}^{4} \mathrm{He}$, ${ }_{\Lambda}^{10} \mathrm{Be},{ }_{\Lambda}^{11} \mathrm{Be}$, and ${ }_{\Lambda}^{19} \mathrm{~F}$ (Tamura et al. 2013). A first result for ${ }_{\Lambda}^{4} \mathrm{He}$ has been obtained (Yamamoto et al., 2015). Lifetimes can be measured using the Doppler shift attenuation method (DSAM) that was first used to extract the lifetime of the $5 / 2^{+}$state of ${ }_{\Lambda}^{7} \mathrm{Li}$, and thus its electromagnetic E2 transition strength $\mathrm{B}(\mathrm{E} 2)$ value (Tanida et al., 2001). Also, the lifetime of the lowest $1 / 2^{+} ; T=1$ state in ${ }_{\Lambda}^{15} \mathrm{~N}$ has been measured (Ukai et al., 2008). Perhaps with the higher intensities provided at J-PARC, the $\Lambda$ magnetic moment in the nuclear medium might also be inferred from measuring the lifetime of M1 transitions between ground-state hypernuclear doublet levels, such as the $\left(3 / 2^{+} \rightarrow 1 / 2^{+}\right) \gamma$ ray in ${ }_{\Lambda}^{7} \mathrm{Li}$ (Tamura et al. 2013). In the weak-coupling limit the strength of the electromagnetic M1 transition $\mathrm{B}(\mathrm{M} 1)$ is proportional to $\left(g_{c}-g_{\Lambda}\right)^{2}$, where $g_{c}$ is the core $g$-factor and $g_{\Lambda}$ is the $\Lambda$ $g$-factor (for the $0 s_{\Lambda}$ orbit in this example). For the simple $\Lambda$-hypernuclear configurations considered here, the 
in-medium $\Lambda \mathrm{g}$-factors could deviate from their corresponding free-space single-particle Schmidt values by at most 10\% (Dover, Feshbach, and Gal 1995; Saito, Oka, and Suzuki, 1997). The lifetime measurement accuracy required to test a few-percent departure of $g_{\Lambda}$ from its Schmidt value can be reached at J-PARC (Tamura et al. 2013).

As the target mass increases to heavier systems the number of both nuclear and hypernuclear $\gamma$ rays increases while the yield to specific hypernuclear states decreases. Although the Doppler shift of in-flight hypernuclear transitions can discriminate between at-rest nuclear transitions, it still becomes more difficult to assign observed $\gamma$ rays to a particular hypernuclear level scheme. Thus, coincident $\gamma$ decays, as well as better resolution of the tagging spectrometer, becomes more important.

The first $\gamma \gamma$ coincidence observation was reported (Ukai et al. 2006), but $\gamma$ coincidences cannot be a widely used tool until production rates are substantially improved. Note that an increase in yield involves more than increasing beam flux, because $\gamma$ detectors are sensitive to backgrounds of all types, and resolution is degraded by rate-dependent electronic pileup.

In addition to $\gamma \gamma$ coincidence measurements, a coincidence between a $\gamma$ and a weak decay can be used to extract information about hypernuclear structure. For hypernuclei with masses up to the middle of the $p$ shell, mesonic, as opposed to non-mesonic, weak decay is sufficiently probable that detection of mono-energetic $\pi^{-}$ emission can be used as a coincidence to tag a specific hypernucleus. If the hypernucleus can be uniquely identified from its mesonic decay, then detection and missingmass analysis of the production reaction would not be necessary, and the observation of $\gamma$ rays from hyperfragments in coincidence with their $\pi^{-}$decay would increase the efficiency of a $\gamma$-ray experiment. The technique also gives access to hypernuclei which could only be produced by fragmentation or nucleon emission; see also the discussion of the Mainz program in Sec. VIII.B.1.

\section{B. Spectroscopy with electron accelerators}

\section{Electroproduction at Mainz (MAMI)}

An ongoing program at the Mainz microtron (MAMI) involves studying the mesonic weak decay of light hypernuclei formed by fragmentation of excited hypernuclear levels reached in electroproduction. This interesting, unexplored, technique uses counters, not emulsion. The microtron energy of $1.5 \mathrm{GeV}$ allows experiments to determine ground-state masses of light hypernuclei by measuring the pion weak decays following the fragmentation of heavier hypernuclear systems reached in kaon electroproduction. For example, ${ }_{\Lambda}^{4} \mathrm{H}$ is strongly produced in $K^{-}$absorption on a number of $p$ -

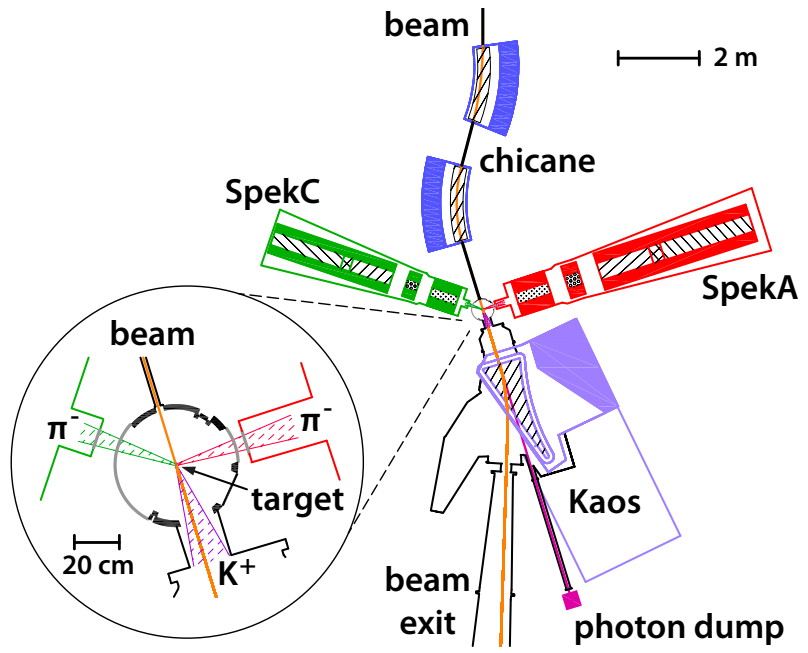

FIG. 42 The spectrometer system at the Mainz microtron designed to observe the pion decay of light hypernuclei formed by fragmentation of heavier hypernuclear systems formed in kaon electroproduction. The KAOS spectrometer detects the kaons emitted in the $\left(e, e^{\prime} K^{+}\right)$reaction, and spectrometers $\mathrm{A}$ and $\mathrm{C}$ detect the decay pions. From Esser et al., 2015.

shell targets and can be identified by the monochromatic $\pi^{-}$'s with $p_{\pi}=133 \mathrm{MeV} / \mathrm{c}$ from the two-body decay ${ }_{\Lambda}^{4} \mathrm{H} \rightarrow{ }^{4} \mathrm{He}+\pi^{-}$(Tamura et al. 1989$)$. In fact, the ${ }_{\Lambda}^{4} \mathrm{H}$ line has been studied recently (Esser et al., 2015) using the setup shown in Fig. 42 [see also (Esser et al. , 2013)] with a ${ }^{9} \mathrm{Be}$ target. The kaon spectrometer (KAOS) detects kaon production with the kaons identified by timeof-flight and an aerogel Cherenkov detector. Spectrometers $\mathrm{A}$ and $\mathrm{C}$ detect the decay pions (spekC for the high-momentum ${ }_{\Lambda}^{4} \mathrm{H}$ line). The binding energy value of $B_{\Lambda}\left({ }_{\Lambda}^{4} \mathrm{H}\right)=2.12 \pm 0.01 \pm 0.09 \mathrm{MeV}$ was obtained and is consistent with the old emulsion value $2.04 \pm 0.04 \mathrm{MeV}$ (cf. Table ID. This is an important result given the importance of establishing precisely the degree of charge-symmetry breaking in the $A=4$ hypernuclei and the fact that the emulsion values for the binding energies were derived from three-body decays because there was no calibration for long-ranged pions in emulsion. For the lower momentum pions typical of $p$-shell hypernuclei, problems certainly exist in assigning the observed pion decay spectrum to specific hypernuclear states. Nevertheless, because the decay of these hypernuclei can be determined by 2-body kinematics, the assignment of masses and binding energies is potentially possible. However, note that mesonic decays from hypernuclear ground states do not necessarily end up in the corresponding daughternuclei ground states (Motoba and Itonaga, 1994; Randeniya and Hungerford, 2007; Gal, 2009).

The use of a $\gamma$-weak decay coincidence has also been proposed to obtain the lifetime of hypernuclear levels that have $\gamma$ lifetimes comparable to those of weak decay (200 ps). This could be used, for example, to measure the $\gamma$ lifetime of the upper level of a hypernuclear ground-state 


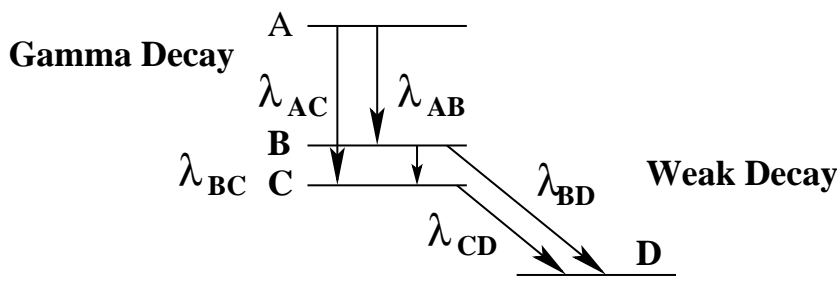

FIG. 43 A schematic illustration of $\gamma$ and weak decay between hypernuclear levels with a ground state doublet $(\mathrm{B}, \mathrm{C})$ having energy spacing $\leq 100 \mathrm{keV}$.

doublet, where the $\gamma$ decay of the upper level competes with weak decay. This generally occurs for high multipolarity transitions of low transition energy, $\leq 100 \mathrm{keV}$. A simultaneous fit to the coincidence times between the weak decays of the doublet levels and the $\gamma$ transitions from $\mathrm{A}$ to $\mathrm{B}$ and $\mathrm{B}$ to $\mathrm{C}$ as shown in the level diagram of Fig. 43 would provide the lifetimes of the B and C levels. Such a program fits into a potential program at Mainz, but the hypernuclei are electroproduced and will be accompanied by significant gamma backgrounds that may preclude $\gamma$-pion coincidence experiments.

\section{Electroproduction at Jlab}

There is substantial, new electroproduced hyperon data from the CLAS detector Collaboration at JLab, particularly polarization and spin transfer data (Carman and Raue, 2009, Dey et al., 2010: McCracken et al., 2010), providing a consistent data base for partial-wave $Y N$ amplitude analysis. The electroproduction of hyperons is a complicated process involving a number of overlapping strange and non-strange resonances (Bydžovský and Skoupil, 2013, Skoupil and Bydžovský, 2016). Whereas $s$-channel diagrams are found to be most important at low energy, $t$-channel/Reggeon exchange dominates when $W>2 \mathrm{GeV}$ (i.e., above the resonance region). More data is expected from CLAS and and also from LEPS at SPring-8 (Niiyama, 2013).

Jlab will be upgraded to a higher energy with more intense beams. The new large solid-angle spectrometers drawn in Fig. 9, HKS and HES, with a new splitting magnet (SPL), will be available. Previously $\left(e, e^{\prime} K^{+}\right)$ hypernuclear programs were undertaken in both Hall A and Hall C. When Jlab transitions to $12 \mathrm{GeV}$ electron beams, hypernuclear experiments will take place in only one Hall. If this is Hall A, a plan exists to design two new septum magnets and move the HKS and the HES from Hall $\mathrm{C}$ into Hall A behind the target station. A waterfall target $\left(\mathrm{H}_{2} \mathrm{O}\right)$ will be retained and could be used to further study the elementary electroproduction amplitude at forward angles and for spectrometer calibrations. By carefully selecting the scattering geometry, bremsstrahlung and Möller backgrounds can be reduced and the lumi- nosity increased to obtain rates of several 10's per hour to specific states. This allows electromagnetic production of hypernuclei through the $s d$ shell with perhaps resolutions approaching $300 \mathrm{keV}$. Proposals have been made for improved energy resolution experiments, after the 12 $\mathrm{GeV}$ upgrade, aiming at the electroproduction of $\Lambda$ hypernuclei beyond the $p$-shell hypernuclei explored so far in Halls A and C (Garibaldi et al., 2013, Tang et al. 2014).

\section{Experiments at PANDA}

The PANDA collaboration using antiprotons at the FAIR future facility in Darmstadt, proposes to produce double- $\Lambda$ hypernuclei, followed by high-resolution $\gamma$-spectroscopy study, in order to provide for the first time precise information on their bound-state spectra (Esser et al. 2013). The PANDA detector is to be set up at the high-energy storage ring (HESR) that produces highintensity phase-space cooled antiprotons with momenta between 1.5 and $15 \mathrm{GeV} / \mathrm{c}$. The antiprotons from the storage ring are extracted and allowed to interact on a nuclear target at $p_{\text {lab }} \approx 3 \mathrm{GeV} / \mathrm{c}$ (Pochodzalla, 2005),

$$
\bar{p}+p \rightarrow \Xi^{-}+\bar{\Xi}^{+}, \quad \bar{p}+n \rightarrow \Xi^{-}+\bar{\Xi}^{0} .
$$

The trigger for these reactions will be based on the detection of high-momentum $\bar{\Xi}$ anti-hyperons at small angles or on $K^{+}$mesons produced by the absorption of antihyperons in the primary target nuclei. Produced $\Xi^{-}$, with typical momenta between 0.5 to $1 \mathrm{GeV} / \mathrm{c}$, are decelerated in a secondary target. The slow $\Xi^{-}$are then either directly absorbed by the nucleus or are captured into an atomic orbit, cascading downward through the $\Xi^{-}$atom levels until absorbed in the $\Xi^{-} p \rightarrow \Lambda \Lambda$ reaction, thereby partially forming a double- $\Lambda$ hypernucleus. X-ray deexcitation between $\Xi$ atomic states, and $\gamma$ deexcitation between states in the $\Lambda \Lambda$ hypernuclei which may be formed, are to be studied with an array of Ge detectors (Pochodzalla, 2005; Esser et al., 2013). One expects to identify approximately 3000 stopped $\Xi^{-}$hyperons per day; see the simulation by Ferro et al. (2007). $\Xi^{-}$capture yields, associated fragmentation mass spectra, and production cross sections of double- $\Lambda$ hypernuclei have been estimated in two recent works (Gaitanos et al. 2012, Gaitanos and Lenske, 2014) using transport in-medium calculations.

\section{Weak decay of hypernuclei}

\section{1. mesonic decays}

Mesonic decays of hypernuclei have been studied since the beginning of hypernuclear experimentation, first in emulsion and more recently in counter experiments at BNL, KEK and by the FINUDA Collaboration 
at DA $\Phi$ NE, Frascati Botta, Bressani, and Garbarino, 2012). A wealth of binding energies and spin-parity values of light $\Lambda$ hypernuclei were deduced in these studies. The well-understood mesonic decay of the $\Lambda$ can be used as a tool to explore nuclear structure when strangeness is injected into the nuclear medium. The pion-decay spectroscopy program at Mainz (Esser et al., 2013), which was reviewed in Sec. VIII.B.1, is poised to develop this tool, primarily by improving the momentum resolution in detecting the emitted pion.

The limitation of mesonic-decay studies to light hypernuclei is due to the low momentum of the recoiling nucleon in the $\Lambda \rightarrow N+\pi$ decay, which is well below the nuclear Fermi momentum $p_{F}$ for $A \geq 6$. However, the $\Lambda$ mesonic-decay rate in the nuclear medium is extremely sensitive to pion distortion effects from in-medium nuclear and electromagnetic interactions. The inclusion of pion-nuclear distortion allows the recoiling nucleon to assume momentum values greater than $p_{F}$, enhancing both $\pi^{0}$ and $\pi^{-}$emission, while Coulomb distortion is expected to raise the $\pi^{-}$decay rates to measurable levels for the heaviest hypernuclei. Indeed, the prediction is that the ratio of the in-medium to free rate saturates at about $10^{-2}$ (Motoba and Itonaga, 1994). However, another calculation, which predicts somewhat similar behavior, results in a rate about a factor of 10 lower in the case of ${ }^{208} \mathrm{~Pb}$ (Oset et al. 1994). There are no available experimental data.

Hypernuclei generally deexcite by $\gamma$ emission to the ground state where they undergo weak decay. In situations where the ground state belongs to a spin doublet based on the nuclear core g.s., weak decay from the upper level can successfully compete with the M1 doublet transition when the transition energy is lower than typically $100 \mathrm{keV}$; see Fig. 43. This may occur in the case of the $\left[1^{-}\right.$(g.s.), $\left.2^{-}\right]$doublet in ${ }_{\Lambda}^{10} \mathrm{~B}$ where no $\gamma$ ray between these two levels has been seen (Chrien et al., 1990; Tamura et al. 2005). Of the two levels, only the $2^{-}$is expected to have been populated in the non-spin-flip production reactions used in these experiments. Therefore, in ${ }_{\Lambda}^{10} \mathrm{~B}$ either the doublet splitting is less than $100 \mathrm{keV}$, thereby hindering the $\gamma$ transition with respect to weak decay, or the level ordering of the spin-doublet members is reversed.

Furthermore, the $\pi^{-}$decay spectrum is substantially different for weak decays from each member of the doublet (Gal, 2009), providing a way to identify the decay sequence. However, in general one might expect a mixture of weak decays from the doublet levels, and a more detailed analysis would be required to extract the decay ratios and determine the ordering. Note that an energy resolution of $\leq 100 \mathrm{keV}$ is required to measure the $\pi^{-}$transition energy shifts in the decays. This may be possible if excellent resolution and sufficient statistics are available. Nevertheless, comparison of the observed pion decay to one calculated for various spin possibilities should allow the level order to be determined.

\section{2. nonmesonic decays}

Of the various observables studied so far, data on nonmesonic weak-decay asymmetries are scarce. Asymmetry and coincident weak-decay experiments are difficult, requiring thick targets, with low yields. A definitive asymmetry experiment would require a substantial increase in intensity and/or polarization, as well as the determination of the polarization of the hypernuclear ground state from which the decay occurs. Better missing-mass resolution to tag ground-state production and the use of a polarizing reaction such as $\left(\pi^{+}, K^{+}\right)$at an angle $>10^{\circ}$ would help, but this requires higher beam intensity.

It would also be important to measure the neutron and proton simulated decays from ${ }_{\Lambda}^{4} \mathrm{H}$ compared to the same decays from ${ }_{\Lambda}^{4} \mathrm{He}$. This comparison would significantly help to resolve the question as to whether the $\Delta I=1 / 2$ rule applies in nonmesonic weak decay $\Lambda+N \rightarrow N+N$ transitions. However, the production of ${ }_{\Lambda}^{4} \mathrm{H}$ requires a charge exchange as well as a strangeness exchange reaction when using a ${ }^{4} \mathrm{He}$ target. Photoproduction is a possibility as well as the $\left(K^{-}, \pi^{0}\right)$ reaction. High beam intensity and large solid angle detectors would be required. A test of the $\Delta I=1 / 2$ rule requires that the final $N N$ states have isospin $I_{f}(N N)=1$, which is reached by the $a, b$, and $f$ amplitudes defined in Table XII. This practically leads to the requirement that the initial $\Lambda N$ state is a purely ${ }^{1} S_{0}$. In this case the $\Delta I=1 / 2$ rule predicts that

$$
\Gamma_{n}\left({ }_{\Lambda}^{4} \mathrm{He}\right)=2 \Gamma_{p}\left({ }_{\Lambda}^{4} \mathrm{H}\right),
$$

which may be tested in the nonmesonic hypernuclear decays of the $A=4$ hypernuclei. The value of the lefthand side $\Gamma_{n}\left({ }_{\Lambda}^{4} \mathrm{He}\right)$ has been determined to be very small, $\Gamma_{n}\left({ }_{\Lambda}^{4} \mathrm{He}\right) / \Gamma_{\Lambda}^{\text {free }} \leq 0.035$ (Parker et al. 2007 ), whereas the value of $\Gamma_{p}\left({ }_{\Lambda}^{4} \mathrm{H}\right)$ is unknown. This will be studied in the J-PARC E22 experiment.

Another area of interest for nonmesonic weak decays would be to study exclusive decay modes, in analogy to the exclusive, two-body mesonic-decay modes of $\Lambda$ hypernuclei that have provided valuable information on spins of $\Lambda$-hypernuclear levels; see Table XI. The study of exclusive decay modes in nonmesonic weak decays could yield valuable information on the $\Lambda+N \rightarrow N+N$ amplitudes of Table XII Examples of such modes in light nuclei are

$$
\begin{gathered}
{ }_{\Lambda}^{5} \mathrm{He} \rightarrow n^{4} \mathrm{He}, d d n, n n^{3} \mathrm{He}, p n^{3} \mathrm{H}, \\
{ }_{\Lambda}^{4} \mathrm{He} \rightarrow p^{3} \mathrm{H}, n^{3} \mathrm{He}, d d, d p n .
\end{gathered}
$$

Rates for some of these decays were measured in bubble chambers and emulsion (Coremans et al. 1970). In 
TABLE XIX ${ }_{\Lambda}^{3} \mathrm{H}$ lifetime (in ps): measurements vs. theory. The free $\Lambda$ lifetime is $(263 \pm 2)$ ps (Olive et al. 2014$)$. The first marked error is statistical, the second one is systematic. $\mathrm{BC}$ denotes a bubble-chamber measurement.

\begin{tabular}{ccccc}
\hline \hline BC $^{\mathrm{a}}$ & STAR $^{\mathrm{b}}$ & HypH] $_{\mid c}^{\mathrm{d}}$ & ALICE $^{\mathrm{d}}$ & Theory \\
\hline $246_{-41}^{+02}$ & $182_{-45}^{+89} \pm 27$ & $183_{-32}^{+42} \pm 37$ & $181_{-39}^{+54} \pm 33$ & 256 \\
\hline \hline
\end{tabular}

a Keyes et al. (1973).

b Abelev et al. (2010).

c Rappold et al. (2013a).

d Adam et al. (2016a).

e Kamada et al. (1998).

passing we mention that the $\Lambda$-hypernuclear program at J-PARC also includes a search for multinucleon emission in the weak decay of hypernuclei, experiment E18 (Takahashi, 2013).

\section{3. $\Lambda$ hypernuclear lifetimes}

Accurate measurements of $\Lambda$-hypernuclear lifetimes in heavy systems beyond $A=56$, as listed in Table VI. could confirm the saturation of the nonmesonic decay width, Eq. 44, as well as provide a check on the $\Gamma_{n} / \Gamma_{p}$ ratio systematics as a function of $A$. Previously, lifetime measurements in delayed fission triggered by proton and antiproton reactions on heavy nuclei, were interpreted as due to the production of $\Lambda$ hypernuclei and their subsequent weak decay. The latest and most accurate measurements of this kind yielded lifetimes [Cassing et al. (2003), Kulessa et al. (1998), and Armstrong et al. (1993), respectively],

$$
\begin{gathered}
\tau_{\Lambda}(p+\mathrm{Au})=(145 \pm 11) \mathrm{ps}, \\
\tau_{\Lambda}(p+\mathrm{Bi})=(161 \pm 7 \pm 14) \mathrm{ps}, \\
\tau_{\Lambda}(\bar{p}+\mathrm{U})=(125 \pm 15) \mathrm{ps} .
\end{gathered}
$$

These are considerably shorter than values extrapolated from Table VI and taken at face value, imply unreasonably large values for $\Gamma_{n} / \Gamma_{p}$ for heavy hypernuclei. Finally, we would like to focus attention again to recent measurements of the ${ }_{\Lambda}^{3} \mathrm{H}$ lifetime in heavy-ion experiments. As reviewed in Sec. I.F.8 the ${ }_{\Lambda}^{3} \mathrm{H}$ lifetime was measured at several heavy-ion facilities using the time dilation of a Lorentz boost to a recoiling hypernucleus produced in a heavy-ion reaction. Lifetimes deduced by the STAR Collaboration at BNL-RHIC, by the HypHI Collaboration at GSI and very recently by the ALICE Collaboration at CERN-LHC (see Fig. 13 in Sec. I.F.8 are listed in Table XIX together with a ${ }_{\Lambda}^{3} \mathrm{H}$ lifetime derived in bubble-chamber studies (Keyes et al., 1973, 1970). The ${ }_{\Lambda}^{3} \mathrm{H}$ lifetime values deduced from measurements made at the heavy-ion facilities are about $25 \%$ shorter than the free $\Lambda$ lifetime, and about $20 \%$ shorter than the value measured in a bubble chamber. Note that the bubble-chamber measurement does not suffer from the uncertainty incurred in emulsion by a possible in-flight Coulomb dissociation of ${ }_{\Lambda}^{3} \mathrm{H}$ (Bohm and Wysotzki, 1970). A recent statistical analysis of all the reported ${ }_{\Lambda}^{3} \mathrm{H}$ lifetime measurements gives an average value $\tau\left({ }_{\Lambda}^{3} \mathrm{H}\right)=\left(216_{-16}^{+19}\right)$ ps (Rappold et al. 2014). A realistic calculation of the lifetime (Kamada et al., 1998) derives a lifetime shorter by only $3 \%$ than the free $\Lambda$ lifetime $\tau_{\Lambda}=(263 \pm 2)$ ps, in agreement with Rayet and Dalitz (1966) that marks the first correct calculation of $\tau\left({ }_{\Lambda}^{3} \mathrm{H}\right)$. The discrepancy between the lifetimes measured in heavy-ion collisions and the lifetime prescribed by theory is disturbing, posing a major problem for the understanding of ${ }_{\Lambda}^{3} \mathrm{H}$, the lightest and hardly bound hypernucleus. More work is necessary to understand the heavy-ion lifetime results. We note that $\tau\left({ }_{\Lambda}^{4} \mathrm{H}\right)$ is also considerably shorter than $\tau_{\Lambda}$, with a world average of $\tau\left({ }_{\Lambda}^{4} \mathrm{H}\right)=192_{-18}^{+20}$ ps (Rappold et al. 2014), but this is theoretically anticipated and well understood.

\section{E. Multi-strange systems}

Nuclear systems with $S=-2$ are essential to experimentally access the hyperon-hyperon interaction. While several light double- $\Lambda$ hypernuclei have been observed, and their phenomenology is fairly well understood (Gal and Millener, 2011), bound $\Xi$ hypernuclei have yet to be observed. Light $\Xi$ hypernuclear systems are predicted to be bound by several $\mathrm{MeV}$, and with sufficiently narrow widths to provide spectroscopy (Hiyama et al. 2008 ). Intense $K^{-}$beams are required for their investigation. The E05 experiment searching for the ${ }_{\Xi}^{12}$ Be hypernucleus (Nagae, 2007) is high on the agenda of J-PARC. The proposal is to use the ${ }^{12} \mathrm{C}\left(K^{-}, K^{+}\right)$reaction to obtain the $1.5 \mathrm{MeV}$ (FWHM) resolution (Takahashi, 2013) that should be sufficient to observe any quasibound structure. $\Xi^{-}$hypernuclear $0^{\circ}$ production cross sections in the bound-state region, using targets in this mass range, are estimated to be a fraction of a $\mu \mathrm{b} / \mathrm{sr}$ (Dover and Gal, 1983; Dover, Gal, and Millener, 1994; Ikeda et al., 1994; Shyam, Tsushima, and Thomas, 2012).

A similar experimental setup is also capable of producing $\Lambda \Lambda$ hypernuclei, either directly or by the conversion $\Xi N \rightarrow \Lambda \Lambda$. Identification of a $\Lambda \Lambda$ hypernucleus could occur either through direct production or by observation of the decay products. In direct production, one would observe the missing mass in a $\left(K^{-}, K^{+}\right)$reaction. In this case, $0^{\circ}$ cross sections are small, a few nb/sr at most (Baltz, Dover, and Millener, 1983; Harada, Hirabayashi, and Umeya, 2010), due to the fact that the reaction requires a multistep interaction on two nucleons. On the other hand, detection in light hypernuclei could occur by observing sequential monoenergetic $\pi^{-}$decays of the embedded $\Lambda$ 's. In either case, good energy resolution 
and tracking is important. All experiments will be difficult because production rates are not expected to be high. A particularly important task would be to settle the question as to whether ${ }_{\Lambda}^{4} \mathrm{H}$ is bound (Filikhin and Gal, 2002c; Nemura, Akaishi, and Myint, 2003). Interest in ${ }_{\Lambda \Lambda}^{4} \mathrm{H}$ arises as it may be the least bound double- $\Lambda \Lambda$ system. A previous experimental claim for the observation of ${ }_{\Lambda \Lambda}^{4} \mathrm{H}$ (Ahn et al. 2001b) is probably incorrect, as shown by a reanalysis of the data Randeniya and Hungerford, 2007).

A possibly strong $\Lambda-\Xi$ attraction in the NSC97 model was pointed out by Filikhin and Gal (2002c). Here the $S=-3$ hypernucleus $\Lambda^{6} \Xi \mathrm{He}$, or $\Lambda \Lambda \Xi{ }^{7} \mathrm{He}$, may provide the onset of $\Xi$ stability in nuclear mater. This observation, and the repulsive nature of the $\Sigma$-nucleus potential, are relevant to the composition of neutron stars, as discussed in Sec. VI.B

\section{F. Experiments at heavy-ion facilities}

Collisions between heavy nuclei $(A \gg 1)$ at relativistic energies produce copiously hadrons and antihadrons, including hyperons and strange mesons. The formation of exotic nuclear systems and their study in relativistic heavy-ion collisions was suggested by Kerman and Weiss (1973). This was further developed by more quantitative evaluations using a variety of production mechanisms (Baltz et al., 1994; Pop and Gupta, 2010; Andronic et al., 2011; Steinheimer et al., 2012). Following collision, the local hadron density produced in the "fireball" stabilizes in times of order $60 \mathrm{fm} / \mathrm{c}$, resulting in the formation of hadronic clusters. These clusters potentially include strange dibaryons, hypernuclei, and other multistrange hadrons. Predictions of production rates use two kinds of models: (i) thermal models in which entropy conservation governs the resulting production yields, following chemical freeze-out at a limiting temperature $T \approx 160 \mathrm{MeV}$ (Andronic et al., 2011), and (ii) coalescense models which apply internuclear cascade simulations of particle collisions and captures, based on particle overlaps in both coordinate and momentum phase space (Steinheimer et al. 2012).

Somewhat surprisingly, the predicted production yields of hypernuclei are model independent above an approximate collision energy of $10 \mathrm{~A} \mathrm{GeV}$, and both types of models predict saturation of the yield at beam energies $\approx 15 \mathrm{~A} \mathrm{GeV}$ (Andronic et al., 2011; Botvina, Gudima, and Pochodzalla, 2013). Dibaryon production, however, is found to be strongly model dependent. These simulation studies demonstrate that $(10-20) \mathrm{A} \mathrm{GeV}$ is the optimal energy for hypernuclear production. Observation of hypernuclear production in relativistic heavy-ion collisions is difficult, and except for light systems, present-day detectors are not really designed to identify and investigate hypernuclear systems of unknown mass and binding

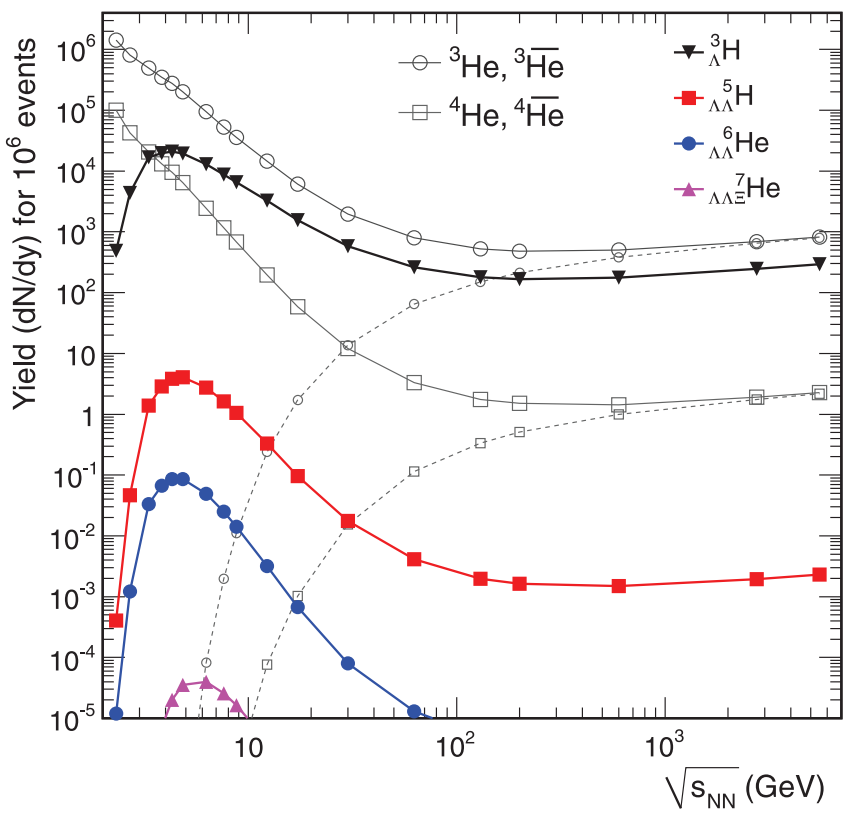

FIG. 44 Energy dependence of predicted yields for several multistrange isotopes of hydrogen and helium at midrapidity for $10^{6}$ heavy-ion central collisions. Predicted yields for two non-strange helium isotopes and their anti-isotopes are also plotted for comparison. From Andronic et al., 2011.

energies. The development of a hypernuclear research program using ion beams of lower mass, e.g., C, with energies of approximately $(10-20) \mathrm{A} \mathrm{GeV}$ would seem appropriate, and can be pursued at the FAIR and NICA facilities (Botvina et al., 2015). Figure 44 illustrates yield predictions for the production of light multistrange hypernuclei at midrapidity per $10^{6}$ central collisions. These thermal-model predictions were constrained by fitting to RHIC hadron production yields at $200 \mathrm{GeV}$.

Focusing on the lightest $A=3,4$ hypernuclei, which are essentially the only ones studied so far in relativistic heavy-ion collisions, the BNL-AGS E864 Collaboration Armstrong et al. 2004) reported the observation of ${ }_{\Lambda}^{3} \mathrm{H}$ in central $\mathrm{Au}+\mathrm{Pt}$ collisions at an energy per $N N$ collision of $\sqrt{s_{N N}}=11.5 \mathrm{GeV}$. Subsequent work by the STAR Collaboration at the BNL-RHIC collider Abelev et al. 2010 identified both ${ }_{\Lambda}^{3} \mathrm{H}$ and its antihypernucleus, ${ }_{\Lambda}^{3} \mathrm{H}$, in $\mathrm{Au}+\mathrm{Au}$ collisions at $\sqrt{s_{N N}}=200 \mathrm{GeV}$. This was followed recently at the CERN-LHC facility by the ALICE Collaboration (Adam et al. $2016 \mathrm{a}$ ) in $\mathrm{Pb}+\mathrm{Pb}$ collisions at $\sqrt{s_{N N}}=2.76 \mathrm{TeV}$. The ${ }_{\Lambda}^{3} \mathrm{H}$ lifetime measurements reported by these heavy-ion experiments were listed and discussed in Table XIX and in the related text.

Searches for exotic nuclear states such as $\Lambda \Lambda$ and $\overline{\Lambda n}$ bound states were also undertaken by the ALICE Collaboration (Adam et al. 2016b), thereby placing upper limits that are typically smaller by one order of magnitude than yields anticipated from thermal models for the production of such states. Another ALICE Col- 
laboration experiment studied the low-energy $\Lambda$ - $\Lambda$ interaction, producing useful constraints on the scattering length and effective range: $a_{\Lambda \Lambda}=-1.10 \pm 0.37_{-0.08}^{+0.68} \mathrm{fm}$ and $r_{\Lambda \Lambda}=8.52 \pm 2.56_{-0.74}^{+2.09} \mathrm{fm}$ (Adamczyk et al. 2015). This result suggests a relatively weak $\Lambda-\Lambda$ interaction, in accord with other existing experimental and theoretical estimates summarized recently by Morita, Furumoto, and Ohnishi (2015).

A program somewhat similar to that of the HypHI Collaboration at GSI (Rappold et al. 2015 ) was proposed for the under-construction nuclotron-based ion collider facility (NICA) at Dubna, using an approximate 3 $\mathrm{GeV} /$ nucleon ${ }^{6} \mathrm{Li}$ beam incident on a ${ }^{\text {nat }} \mathrm{C}$ target. A more sophisticated trigger would be based on identifying the recoiling hypernuclei by using a new magnetic spectrometer to measure the momentum of their two-body pionic decays. The pions and residual particles from the decays would be detected with multiwire proportional chambers placed behind the spectrometer magnet to reconstruct the hypernuclei from their decay products, which were presumed to be ${ }_{\Lambda}^{A} \mathrm{H} \rightarrow{ }^{A} \mathrm{He}+\pi^{-}$or ${ }_{\Lambda}^{A} \mathrm{He} \rightarrow{ }^{A} \mathrm{Li}+\pi^{-}$(Averyanov et al. 2008). The main interest in this program would be the potential production of light, neutron-rich hypernuclei inaccessible by other reactions. However, obtaining lifetimes of heavy hypernuclei, where mesonic decay is suppressed and essentially unobservable, is more compelling at present.

\section{G. $\bar{K}$-nucleus bound-state searches}

The topic of $\mathrm{K}^{-}$-nuclear bound states has generated much heat and perhaps little illumination. Experimental searches for these states using stopped kaon reactions with outgoing neutrons, at KEK, or protons, at DA $\Phi$ NE, at first suggested bound-state structure at more than $100 \mathrm{MeV}$ below threshold. However, the KEK observation (Suzuki et al. 2004, 2005) of a $\bar{K} N N N$ structure is now believed to be an experimental artifact, and at least a large part of the FINUDA Collaboration observation of a $K^{-} p p$ structure at DA $\Phi$ NE $(\mathrm{Ag}-$ nello et al., 2005a must be due to final-state interactions (Magas et al., 2006). Yet the theoretical prediction of a $K^{-} p p$ bound state is reasonably robust, with microscopic preference for shallow binding of few tens of MeV (Gal, 2013). Recent searches by the HADES Collaboration using the $p p \rightarrow \Lambda p K^{+}$reaction at GSI and performing a complete background evaluation (Epple and Fabbietti, 2015) have refuted earlier claims for a deeply bound $K^{-} p p$ state based on a DISTO Collaboration analysis of older proton-beam data (Yamazaki et al. 2010). In addition, the LEPS Collaboration at SPring8 also published upper limits, although less significant than with meson beams, for the production of a $K^{-} p p$ bound state via the $d\left(\gamma, K^{+} \pi^{-}\right)$reaction at photon energy $E_{\gamma}=1.5-2.4 \mathrm{GeV}$ (Tokiyasu et al. 2014 ).
Ongoing experiments at J-PARC using meson beams reach contradictory results. E27 claims to have observed a deeply bound $K^{-} p p$-like structure in the $d\left(\pi^{+}, K^{+}\right)$ reaction at $p_{\pi}=1.69 \mathrm{GeV} / \mathrm{c}$ (Ichikawa et al. , 2015), whereas E15 presented upper limits in the ${ }^{3} \mathrm{He}\left(\overline{K^{-}, n}\right)$ reaction at $p_{K}=1 \mathrm{GeV} / \mathrm{c}$ (Hashimoto et al., 2015) that appear to rule out a $K^{-} p p$ bound state with binding energy similar to that claimed by E27. However, E15, by focusing on the detection of $\Lambda p$ pairs, now suggests a broad $K^{-} p p$ bound-state structure at just $15 \mathrm{MeV}$ below threshold (Sada et al., 2016). This ambiguity in identifying broad $K$-nuclear bound-state structures reflects an experimental difficulty to directly access the formation and decay of such kaonic bound states. In particular, the detector used in such experiments must have good resolution, particle identification, and large angular acceptance. Further, improved experimentation searching for $\bar{K}$-nucleus bound-state structures is required to settle this issue.

\section{SUMMARY}

Strangeness nuclear physics has been invesitgated since the first hyperon (the $\Lambda$ ) was observed in cosmic rays. Progress in this field has not been rapid but continuous, with its development critically dependent on both the experimental and theoretical tools to fully exploit the physics. The previous sections reviewed the production mechanisms with which $\Lambda$ and $\Sigma$ hyperons are injected into the nuclear medium. In addition, multistrangeness and the hyperon puzzle in neutron stars were reviewed, along with the strong interaction of $\bar{K}$ mesons in and with nuclei, including the possibility to form $\bar{K}$-nuclear quasibound states. The nonmesonic weak decay of hypernuclei offers the unique opportunity to study the four-fermion weak interaction, and in particular, the fundamental origin (if any) of the empirical $\Delta \mathrm{I}=1 / 2$ rule. A number of potential experimental areas which seem critical for further advances in this field were pointed out.

To highlight obvious achievements in strangeness nuclear physics and outstanding problems facing this field of research for the coming years, a brief, perhaps subjective list follows:

- With the $\Lambda N$ hypernuclear spin dependence largely deciphered via $\gamma$-ray studies, why is the $\Lambda$-nuclear spin-orbit splitting so small?

- What is the role of three-body $\Lambda N N$ interactions in hypernuclei and at neutron-star densities?

- The $\Sigma$-nuclear interaction is established as being repulsive, but how repulsive?

- Where is the onset of $\Lambda \Lambda$ binding: ${ }_{\Lambda \Lambda}^{4} \mathrm{H}$ or ${ }_{\Lambda \Lambda}^{5} \mathrm{H}$ and ${ }_{\Lambda \Lambda}^{5} \mathrm{He}$ ? 
TABLE XX J-PARC scheduled experiments related to strangeness nuclear physics. DCX stands for double-charge exchange and TES for transition-edge sensor.

\begin{tabular}{lll}
\hline \hline Exp. & \multicolumn{1}{c}{ Title } & \multicolumn{1}{c}{ Status } \\
\hline E03 & X rays from $\Xi^{-}$atoms & Day-1 experiment \\
E05 & ${ }_{12} \mathrm{C}\left(K^{-}, K^{+}\right)_{\Xi}^{12}$ Be & \\
E07 & $\mathrm{S}=-2$ emulsion-counter studies & Negative result for ${ }_{4}^{6} \mathrm{H}$ \\
E10 & DCX studies of neutron-rich ${ }_{\Lambda}^{A} Z$ & Day-1 experiment, ${ }_{\Lambda} \mathrm{He} \gamma$ ray observed \\
E13 & $\gamma$-ray spectroscopy of $\Lambda$ hypernuclei & Day-1 experiment, shallow $K^{-} p p$ \\
E15 & Search for $K^{-} p p$ in ${ }^{3} \mathrm{He}\left(K^{-}, n\right)$ & bound state suggested \\
& & \\
E18 & ${ }_{\Lambda} \mathrm{C}$ weak decays & Day-1 experiment, upper bound established \\
E19 & Search for $\Theta^{+}$pentaquark in $\pi^{-} p \rightarrow K^{-} X$ & \\
E22 & Weak interactions in ${ }_{\Lambda}^{4} \mathrm{H}-{ }_{\Lambda}^{4} \mathrm{He}$ & Deeply-bound " $K^{-} p p$-like" \\
E27 & Search for $K^{-} p p$ in $d\left(\pi^{+}, K^{+}\right)$ & bound state claimed \\
E31 & Study of $\Lambda(1405)$ by in-flight $d\left(K^{-}, n\right)$ & \\
E40 & Measurement of $\Sigma p$ scattering & \\
E42 & Search for $H$-dibaryon in $\left(K^{-}, K^{+}\right)$nuclear reactions & Supersedes old day-1 experiment E17 \\
E62 & Precision spectroscopy of X-rays from kaonic atoms with TES & \\
\hline \hline
\end{tabular}

- Do $\Xi$ hyperons bind in nuclei and how broad are the single-particle levels given the $\Xi N \rightarrow \Lambda \Lambda$ strong decay channel?

- Where is the onset of $\Xi$ stability: ${ }_{\Lambda \Xi}{ }^{6} \mathrm{He}$ or ${ }_{\Lambda \Lambda}{ }_{\Xi}^{7} \mathrm{He}$ ?

- Although no $\bar{K}$ condensation occurs in self-bound stable matter, can one observe $\bar{K}$ bound states in spite of the expected large widths $\Gamma \geq 50 \mathrm{MeV}$ (for example, $\left.K^{-} p p\right)$ ?

- Is strange hadronic matter, made of roughly equal amounts of nucleons, $\Lambda$ and $\Xi$ hyperons, likely to provide the ground state of strange matter?

The field is now poised to begin exploiting the new programs proposed at J-PARC, MAMI, FAIR, and at the upgraded JLab. These programs take advantage of new detection and electronic technologies which allow higher rates and coincidence experiments. To demonstrate the richness of the experimental programs we list in Table $\mathrm{XX}$ the J-PARC scheduled experiments which, obviously, are limited to meson beams but still cover a broad spectrum of strangeness nuclear physics topical issues.

\section{ACKNOWLEDGMENTS}

This work was supported by the U.S. DOE under Contract Nos. de-sc0012704 (D. J. M.) and de-sc0011598 (E. V. H.).

\section{REFERENCES}

Abelev, B. I., et al., 2010, Science 328, 58. Adam, J., et al., 2016a, Phys. Lett. B 754, 360. Adam, J., et al., 2016b, Phys. Lett. B 752, 267.
Adamczyk, L., et al., 2015, Phys. Rev. Lett. 114, 022301. Agnello, M., et al., 2005a, Phys. Rev. Lett. 94, 212303. Agnello, M., et al., 2005b, Phys. Lett. B 622, 35. Agnello, M., et al., 2008, Nucl. Phys. A 804, 151. Agnello, M., et al., 2009, Phys. Lett. B 681, 139. Agnello, M., et al., 2011a, Phys. Lett. B 704, 474. Agnello, M., et al., 2011b, Phys. Lett. B 698, 219. Agnello, M., et al., 2011c, Phys. Lett. B 701, 556. Agnello, M., et al., 2012a, Phys. Rev. Lett. 108, 042501. Agnello, M., et al., 2012b, Nucl. Phys. A 881, 322. Agnello, M., et al., 2012c, Nucl. Phys. A 881, 269. Agnello, M., et al., 2014, Phys. Lett. B 738, 499. Agnello, M., et al., 2015, Phys. Rev. C 92, 045204. Ahmed, M. W., et al., 2003, Phys. Rev. C 68, 064004. Ahn, J. K., et al., 1996, Phys. Lett. B 378, 53.

Ahn, J. K., et al., 1999, Nucl. Phys. A 648, 263.

Ahn, J. K., et al., 2001a, in Hadrons and Nuclei: First International Symposium, Seoul, Korea, edited by S.-W. Hong, I.-T. Cheon, T. Choi, and S. H. Lee, AIP Conf. Proc. Vol. 594 (AIP, New York), p. 180.

Ahn, J. K., et al., 2001b, Phys. Rev. Lett. 87, 132504.

Ahn, J. K., et al., 2013, Phys. Rev. C 88, 014003.

Ajimura, S., et al., 2001, Phys. Rev. Lett. 86, 4255.

Akaishi, Y., T. Harada, S. Shinmura, and K. S. Myint, 2000, Phys. Rev. Lett. 84, 3539.

Akikawa, H., et al., 2002, Phys. Rev. Lett. 88, 082501.

Alberico, W. M., A. De Pace, M. Ericson, and A. Molinari, 1991, Phys. Lett. B 256, 134.

Alexander, G., U. Karshon, A. Shapira, G. Yekutieli, R. Engelmann, H. Filthuth, and W. Lughofer, 1968, Phys. Rev. 173, 1452.

Alford, M., M. Braby, M. Paris, and S. Reddy, 2005, Astrophys. J. 629, 969.

Alston, M., et al., 1961, Phys. Rev. Lett. 6, 698.

Ammar, R., W. Dunn, and M. Holland, 1962, Nuovo Cimento 26, 840 .

Ammar, R., R. Levi Setti, W. Slater, S. Limentani, P. Schlein, and P. Steinberg, 1961, Nuovo Cimento 19, 20.

Andronic, A., P. Braun-Munzinger, J. Stachel, and H. Stöcker, 2011, Phys. Lett. B 697, 203. 
Antoniadis, J., et al., 2013, Science 340, 1233232.

Aoki, S., et al., 1991, Prog. Theor. Phys. 85, 1287.

Aoki, S., et al., 1993, Prog. Theor. Phys. 89, 493.

Aoki, S., et al., 1995, Phys. Lett. B 355, 45.

Aoki, S., et al., 2009, Nucl. Phys. A 828, 191.

Armstrong, T. A., et al., 1993, Phys. Rev. C 47, 1957.

Armstrong, T. A., et al., 2004, Phys. Rev. C 70, 024902.

Auerbach, E. H., A. J. Baltz, C. B. Dover, A. Gal., S. H.

Kahana, L. Ludeking, and D. J. Millener, 1981, Phys. Rev.

Lett. 47, 1110.

Auerbach, E. H., A. J. Baltz, C. B. Dover, A. Gal., S. H. Kahana, L. Ludeking, and D. J. Millener, 1983, Ann. Phys. (N.Y.) 148, 381.

Averyanov, A. V., et al., 2008, Phys. At. Nucl. 71, 2101.

Baca, A., C. Garcia-Recio, and J. Nieves, 2000, Nucl. Phys. A 673, 335.

Balberg, S., and A. Gal, 1997, Nucl. Phys. A 625, 435.

Balberg, S., A. Gal, and J. Schaffner, 1994, Prog. Theor. Phys. Suppl. 117, 325.

Baltz, A. J., C. B. Dover, S. H. Kahana, Y. Pang, T. J. Schlagel, and E. Schnedermann, 1994, Phys. Lett. B 325, 7.

Baltz, A. J., C. B. Dover, and D. J. Millener, 1983, Phys. Lett. B 123, 9.

Bamberger, A., et al., 1973, Nucl. Phys. B 60, 1.

Bandō, H., and T. Motoba, 1986, Prog. Theor. Phys. 76, 1321.

Bandō, H., T. Motoba, M. Sotona, and J. Žofka, 1989, Phys. Rev. C 39, 587.

Barbero, C., C. De Conti, A. P. Galeão, and F. Krmpotić, 2003, Nucl. Phys. A 726, 267.

Barbero, C., and A. Mariano, 2006, Phys. Rev. C 73, 024309.

Barnea, N., A. Gal, and E. Liverts, 2012, Phys. Lett. B 712, 132.

Barret, D., J.-F. Olive, and M. C. Miller, 2006, Mon. Not. Roy. Astron. Soc. 370, 1140.

Bart, S., et al., 1999, Phys. Rev. Lett. 83, 5238.

Batty, C. J., 1979, Phys. Lett. B 87, 324.

Batty, C. J., E. Friedman, and A. Gal, 1994a, Phys. Lett. B 335, 273.

Batty, C. J., E. Friedman, and A. Gal, 1994b, Prog. Theor. Phys. Suppl. 117, 227.

Batty, C. J., E. Friedman, and A. Gal, 1997, Phys. Rep. 287, 385.

Batty, C. J., E. Friedman, and A. Gal, 1999, Phys. Rev. C 59, 295.

Bauer, E., and G. Garbarino, 2010, Phys. Rev. C 81, 064315.

Bauer, E., and G. Garbarino, 2012, Phys. Rev. C 85, 024321.

Bauer, E., G. Garbarino, A. Parreño, and A. Ramos, 2010, Nucl. Phys. A 836, 199.

Baym, G. A., and S. A. Chin, 1976, Phys. Lett. B 62, 241.

Bazzi, M., et al., 2011, Phys. Lett. B 704, 113.

Bazzi, M., et al., 2012, Nucl. Phys. A 881, 88.

Beane, S. R., et al., 2011, Phys. Rev. Lett. 106, 162001.

Bedjidian, M., et al., 1979, Phys. Lett. B 83, 252.

Belyaev, V. B., S. A. Rakityansky, and W. Sandhas, 2008, Nucl. Phys. A 803, 210.

Bender, S., R. Shyam, and H. Lenske, 2010, Nucl. Phys. A 839, 51.

Berger, M. S., and R. L. Jaffe, 1987, Phys. Rev. C 35, 213.

Bertini, R., et al., 1979, Phys. Lett. B 83, 306.

Bertini, R., et al., 1980, Phys. Lett. B 90, 375.

Bertini, R., et al., 1981, Nucl. Phys. A 360, 315.

Bertini, R., et al., 1984, Phys. Lett. B 136, 29.
Bertini, R., et al., 1985, Phys. Lett. B 158, 19.

Bertrand, D., et al., 1970, Nucl. Phys. B 16, 77.

Bhang, H., et al., 1998, Phys. Rev. Lett. 81, 4321.

Block, M. M., and R. H. Dalitz, 1963, Phys. Rev. Lett. 11, 96.

Block, M. M., L. Lendinara, and L. Monari, 1962, in Proc. Int. Conf. High-Energy Phys., CERN, 1962, edited by J. Prentki (CERN, Geneva), p. 371.

Block, M. M., et al., 1964, in Proc. Int. Conf. Hyperfragments, St. Cergue, 1963, edited by W. Lock (CERN, Geneva), p. 63.

Bocquet, J. P., et al., 1987, Phys. Lett. B 192, 312.

Bodmer, A. R., 1971, Phys. Rev. D 4, 1601.

Bodmer, A. R., and Q. N. Usmani, 1988, Nucl. Phys. A 477, 621.

Bodmer, A. R., Q. N. Usmani, and J. Carlson, 1984a, Phys. Rev. C 29, 684.

Bodmer, A. R., Q. N. Usmani, and J. Carlson, 1984b, Nucl. Phys. A 422, 510.

Bohm, G., and F. Wysotzki, 1970, Nucl. Phys. B 15, 628.

Bohm, G., et al., 1968, Nucl. Phys. B 4, 511.

Bohm, G., et al., 1969, Nucl. Phys. B 9, 1, and references cited therein.

Bohm, G., et al., 1970a, Nucl. Phys. B 16, 46.

Bohm, G., et al., 1970b, Nucl. Phys. B 23, 93.

Bonazzola, G. C., et al., 1974, Phys. Lett. B 53, 297.

Botta, E., T. Bressani, and G. Garbarino, 2012, Eur. Phys. J. A 48, 41.

Botvina, A. S., K. K. Gudima, and J. Pochodzalla, 2013, Phys. Rev. C 88, 054605.

Botvina, A. S., J. Steinheimer, E. Bratkovskaya, M. Bleicher, and J. Pochodzalla, 2015, Phys. Lett. B 742, 7.

Bouyssy, A., 1977, Nucl. Phys. A 290, 324.

Bouyssy, A., 1980, Phys. Lett. B 91, 15.

Boyle, P. A., et al., 2013, Phys. Rev. Lett. 110, 152001.

Brückner, W., et al., 1975, Phys. Lett. B 55, 107.

Brückner, W., et al., 1978, Phys. Lett. B 79, 157.

Bydžovský, P., A. Gal, and J. Mareš, 2007, Eds., Topics in strangeness physics, Lecture Notes in Physics, Vol. 724 (Springer, Berlin/Heidelberg).

Bydžovský, P., and D. Skoupil, 2013, Nucl. Phys. A 914, 14.

Cantwell, T., et al., 1974, Nucl. Phys. A 236, 445.

Carman, D. S., and B. A. Raue, 2009, Phys. Rev. C 79, 065205 .

Cassing, W., et al., 2003, Eur. Phys. J. A 16, 549.

Chatterjee, D., and I. Vidaña, 2016, Eur. Phys. J. A 52, 29.

Cheston, W. B., and H. Primakoff, 1953, Phys. Rev. 92, 1537.

Chin, S. A., and A. K. Kerman, 1979, Phys. Rev. Lett. 43, 1292.

Chrien, R. E., et al., 1979, Phys. Lett. B 89, 31.

Chrien, R. E., et al., 1990, Phys. Rev. C 41, 1062.

Chumillas, C., G. Garbarino, A. Parreño, and A. Ramos, 2007, Phys. Lett. B 657, 180.

Chumillas, C., G. Garbarino, A. Parreño, and A. Ramos, 2008, Nucl. Phys. A 804, 162.

Cieplý, A., E. Friedman, A. Gal, and V. Krejčiřík, 2011, Phys. Lett. B 698, 226.

Cieplý, A., E. Friedman, A. Gal, and J. Mareš, 2001, Nucl. Phys. A 696, 173.

Cieplý, A., E. Friedman, A. Gal, and J. Mareš, 2003, Nucl. Phys. A 721, C975.

Cieplý, A., and A. Gal, 1997, Phys. Rev. C 55, 2715.

Cieplý, A., and J. Smejkal, 2012, Nucl. Phys. A 881, 115.

Clayton, E. F., et al., 1975, Nucl. Phys. B 95, 130. 
Cohen, S., and D. Kurath, 1965, Nucl. Phys. A 73, 1.

Coremans, G., J. Sacton, D. O'Sullivan, F. Esmael, D. H. Davis, M. A. Shaukat, T. Pniewski, and J. E. Allen, 1970, Nucl. Phys. B 16, 209.

Cugnon, J., A. Lejeune, and H.-J. Schulze, 2000, Phys. Rev. C 62, 064308.

Cusanno, F., et al., 2009, Phys. Rev. Lett. 103, 202501.

Dabrowski, J., 1999, Phys. Rev. C 60, 025205.

Dalitz, R. H., 1958, Phys. Rev. 112, 605.

Dalitz, R. H., 1963a, Nucl. Phys. 41, 78.

Dalitz, R. H., 1963b, Phys. Lett. 5, 53.

Dalitz, R. H., 1969, in Nuclear Physics, edited by C. DeWitt and V. Gillet (Gordon and Breach, New York), p. 701.

Dalitz, R. H., 1973, in Summer Study Meeting on Nuclear and Hypernuclear Physics with Koan Beams, edited by H. Palevsky (BNL Report 18335, Upton, NY), p. 1.

Dalitz, R. H., 2005, Nucl. Phys. A 754, 14c.

Dalitz, R. H., D. H. Davis, P. H. Fowler, A. Montwill, J. Pniewski, and J. Zakrzewski, 1989, Proc. Roy. Soc. London A 426, 1.

Dalitz, R. H., D. H. Davis, and D. N. Tovee, 1986, Nucl. Phys. A 450, 311c.

Dalitz, R. H., and A. Gal, 1976, Phys. Lett. B 64, 154.

Dalitz, R. H., and A. Gal, 1978, Ann. Phys. (N.Y.) 116, 167.

Dalitz, R. H., and A. Gal, 1981, Ann. Phys. (N.Y.) 131, 314.

Dalitz, R. H., R. C. Herndon, and Y. C. Tang, 1972, Nucl. Phys. B 47, 109.

Dalitz, R. H., and F. von Hippel, 1964a, Phys. Lett. 10, 153.

Dalitz, R. H., and F. von Hippel, 1964b, Nuovo Cimento 34, 799.

Dalitz, R. H., and L. Liu, 1959, Phys. Rev. 116, 1312.

Dalitz, R. H., and F. S. Tuan, 1959, Phys. Rev. Lett. 2, 425.

Dalitz, R. H., T. C. Wong, and G. Rajasekaran, 1967, Phys. Rev. 153, 1617.

Danysz, M., and J. Pniewski, 1953, Phil. Mag. 44, 348.

Danysz, M., et al., 1963a, Nucl. Phys. 49, 121.

Danysz, M., et al., 1963b, Phys. Rev. Lett. 11, 29.

Davis, D. H., 1991, in LAMPF Workshop on $(\pi, K)$ Physics, edited by B. F. Gibson, W. R. Gibbs, and M. B. Johnson, AIP Conf. Proc. Vol. 224 (AIP, New York), p. 38.

Davis, D. H., 2005, Nucl. Phys. A 754, 3c.

Davis, D. H., 2008, Nucl. Phys. A 804, 5.

Davis, D. H., R. Levi Setti, and M. Raymund, 1963, Nucl. Phys. 41, 73.

Davis, D. H., and J. Pniewski, 1986, Contemp. Phys. 27, 91.

Demorest, P., et al., 2010, Nature (London) 467, 1081.

Dey, B., C. A. Meyer, M. Bellis, M. E. McCracken, and M. Williams, 2010, Phys. Rev. C 82, 025202.

Djapo, H., B.-J. Schaefer, and J. Wambach, 2010, Phys. Rev. C 81, 035803.

Dłuzewski, P., K. Garbowska-Pniewska, J. Pniewski, Y. Tymieniecka, P. Cook, and D. H. Davis, 1988, Nucl. Phys. A 484, 520.

Dohrmann, F., et al., 2007, Phys. Rev. C 76, 054004.

Doté, A., T. Hyodo, and W. Weise, 2008, Nucl. Phys. A 804, 197.

Doté, A., T. Hyodo, and W. Weise, 2009, Phys. Rev. C 79, 014003.

Dover, C. B., H. Feshbach, and A. Gal, 1995, Phys. Rev. C $\mathbf{5 1}, 541$.

Dover, C. B., and A. Gal, 1983, Ann. Phys. (NY) 146, 309.

Dover, C. B., and A. Gal, 1984, Prog. Part. Nucl. Phys. 12, 171.
Dover, C. B., A. Gal, and D. J. Millener, 1984, Phys. Lett. B 138, 337.

Dover, C. B., A. Gal, and D. J. Millener, 1994, Nucl. Phys. A 572, 85 .

Dover, C. B., L. Ludeking, and G. E. Walker, 1980, Phys. Rev. C 22, 2073.

Dover, C. B., D. J. Millener, and A. Gal, 1989, Phys. Rep. $184,1$.

Dover, C. B., D. J. Millener, A. Gal, and D. H. Davis, 1991, Phys. Rev. C 44, 1905.

Downs, B. W., and R. H. Dalitz, 1959, Phys. Rev. 114, 593.

Eisele, F., H. Filthuth, W. Föhlisch, V. Hepp, and G. Zech, 1971, Phys. Lett. B 37, 204.

Epelbaum, E., H.-W. Hammer, and U.-G. Meißner, 2009, Rev. Mod. Phys. 81, 1773.

Epple, E., and L. Fabbietti, 2015, Phys. Rev. C 92, 044002.

Esser, A., et al., 2013, Nucl. Phys. A 914, 519.

Esser, A., et al., 2015, Phys. Rev. Lett. 114, 232501.

Faessler, M. A., et al., 1973, Phys. Lett. B 46, 468.

Farhi, E., and R. L. Jaffe, 1984, Phys. Rev. D 30, 2379.

Feliciello, A., and T. Nagae, 2015, Rep. Prog. Phys. 78, 096301.

Ferro, F., M. Agnello, F. Iazzi, and K. Szymańska, 2007, Nucl. Phys. A 789, 209.

Fetisov, V. N., L. Majling, J. Žofka, and R. A. Eramzhyan, 1991, Z. Phys. A 339, 399.

Fetkovich, J. G., et al., 1972, Phys. Rev. D 6, 3069, and references cited therein.

Filikhin, I. N., and A. Gal, 2002a, Nucl. Phys. A 707, 491.

Filikhin, I. N., and A. Gal, 2002b, Phys. Rev. Lett. 89, 172502.

Filikhin, I. N., and A. Gal, 2002c, Phys. Rev. C 65, 041001.

Filikhin, I. N., A. Gal, and V. M. Suslov, 2003, Phys. Rev. C 68, 024002.

Finelli, P., N. Kaiser, D. Vretenar, and W. Weise, 2009, Nucl. Phys. A 831, 163.

Freire, P. C., et al., 2009, arXiv:0902.2891.

Friedman, E., and A. Gal, 2007, Phys. Rep. 452, 89.

Friedman, E., and A. Gal, 2012, Nucl. Phys. A 881, 150.

Friedman, E., and A. Gal, 2013, Nucl. Phys. A 899, 60.

Friedman, E., and S. Okada, 2013, Nucl. Phys. A 915, 170.

Fujiwara, Y., M. Kohno, and Y. Suzuki, 2007a, Nucl. Phys. A 784, 161.

Fujiwara, Y., Y. Suzuki, and C. Nakamoto, 2007b, Prog. Part. Nucl. Phys. 58, 439, and refereces cited therein.

Fukuda, T., et al., 1995, Nucl. Intrum. Methods Phys. Res., Sect. A 361, 485.

Fukuda, T., et al., 1998, Phys. Rev. C 58, 1306.

Gaitanos, T., A. B. Larionov, H. Lenske, and U. Mosel, 2012, Nucl. Phys. A 881, 240.

Gaitanos, T., and H. Lenske, 2014, Phys. Lett. B 737, 256.

Gal, A., 2009, Nucl. Phys. A 828, 72.

Gal, A., 2010, Prog. Theor. Phys. Suppl. 186, 270.

Gal, A., 2013, Nucl. Phys. A 914, 270.

Gal, A., 2015, Phys. Lett. B 744, 352.

Gal, A., and C. B. Dover, 1980, Phys. Rev. Lett. 44, 379.

Gal, A., E. Friedman, N. Barnea, A. Cieplý, J. Mareš, and D. Gazda, 2014, Acta Phys. Polon. B 45, 673.

Gal, A., and H. Garcilazo, 2013, Nucl. Phys. A 897, 167.

Gal, A., and H. Garcilazo, 2014, Phys. Lett. B 736, 93.

Gal, A., O. Hashimoto, and J. Pochodzalla, 2012, Eds., Progress in strangeness nuclear physics, Nucl. Phys. A 881, 1-338. 
Gal, A., and R. S. Hayano, 2008, Eds., Recent advances in strangeness nuclear physics, Nucl. Phys. A 804, 1-348.

Gal, A., and E. Hungerford, 2005, Eds., Proceedings of the 8th international conference on hypernuclear and strange particle physics, Nucl. Phys. A 754, 1-490.

Gal, A., and L. Klieb, 1986, Phys. Rev. C 34, 956.

Gal, A., and D. J. Millener, 2011, Phys. Lett. B 701, 342.

Gal, A., and D. J. Millener, 2013, Phys. Lett. B 725, 445.

Gal, A., J. M. Soper, and R. H. Dalitz, 1971, Ann. Phys. (N.Y.) 63, 53.

Gal., A., J. M. Soper, and R. H. Dalitz, 1972, Ann. Phys. (N.Y.) 72, 445.

Gal., A., J. M. Soper, and R. H. Dalitz, 1978, Ann. Phys. (N.Y.) 113, 79.

Gandolfi, S., and D. Lonardoni, 2015, arXiv:1512.06832.

Garbarino, G., A. Parreño, and A. Ramos, 2004, Phys. Rev. C 69, 054603.

Garcilazo, H., and A. Valcarce, 2014, Phys. Rev. C 89, 057001 .

Garibaldi, F., et al., 2013, Nucl. Phys. A 914, 34.

Gazda, D., E. Friedman, A. Gal, and J. Mareš, 2008, Phys. Rev. C 77, 045206.

Gazda, D., and A. Gal, 2016, Phys. Rev. Lett. 116, 122501.

Gazda, D., J. Mareš, P. Navrátil, R. Roth, and R. Wirth, 2014, Few-Body Syst. 55, 857.

Gazda, D., and J. Mareš, 2012, Nucl. Phys. A 881, 159.

Gell-Mann, M., 1953, Phys. Rev. 92, 833.

Gibbs, W. R., S. A. Coon, H. K. Han, and B. F. Gibson, 2000, Phys. Rev. C 61, 064003.

Gibson, B. F., and E. V. Hungerford, 1995, Phys. Rep. 257, 349.

Gibson, B. F., K. Imai, T. Motoba, T. Nagae, and A. Onishi, 2010, Eds., Proceedings of the 10th international conference on hypernuclear and strange particle physics, Nucl. Phys. A 835, 1-470.

Gibson, B. F., and D. R. Lehman, 1979, Nucl. Phys. A 329, 308.

Gibson, B. F., and D. R. Lehman, 1988, Phys. Rev. C 37, 679.

Gibson, B. F., and R. G. E. Timmermans, 1998, Nucl. Phys. A 628, 417.

Gilson, E. P., and R. L. Jaffe, 1993, Phys. Rev. Lett. 71, 332.

Glendenning, N. K., 1985, Astrophys. J. 293, 470.

Glendenning, N. K., 2001, Phys. Rev. C 64, 025801.

Glendenning, N. K., and J. Schaffner-Bielich, 1999, Phys. Rev. C 60, 025803.

Gogami, T., 2014, Spectroscopic research of $\Lambda$ hypernuclei up to medium-heavy mass region with the $\left(e, e^{\prime} K^{+}\right)$reaction, Ph.D. thesis, Tohoku University, Sendai, Japan.

Gogami, T., et al., 2016a, Phys. Rev. C 93, 034314.

Gogami, T., et al., 2016b, Phys. Rev. C 94, 021302(R).

Gross, E. E., E. V. Hungerford, and J. J. Malanify, 1971, Nucl. Phys. A 164, 376.

Guo, Z. H., and J. A. Oller, 2013, Phys. Rev. C 87, 035202.

Haidenbauer, J., 2013, Nucl. Phys. A 914, 220.

Haidenbauer, J., and U.-G. Meißner, 2005, Phys. Rev. C 72, 044005.

Haidenbauer, J., and U.-G. Meißner, 2012, Nucl. Phys. A 881, 44.

Haidenbauer, J., and U.-G. Meißner, 2015, Nucl. Phys. A 936 , 29.

Haidenbauer, J., U.-G. Meißner, and S. Petschauer, 2016, Nucl. Phys. A 954, 273.
Haidenbauer, J., S. Petschauer, N. Kaiser, U.-G. Meißner, A. Nogga, and W. Weise, 2013, Nucl. Phys. A 915, 24.

Halderson, D., 1988, Phys. Rev. C 37, 1104.

Halderson, D., 2008, Phys. Rev. C 77, 034304.

Harada, T., 1998, Phys. Rev. Lett. 81, 5287.

Harada, T., 2001, Nucl. Phys. A 691, 68c.

Harada, T., and Y. Hirabayashi, 2005, Nucl. Phys. A 759, 143.

Harada, T., and Y. Hirabayashi, 2006, Nucl. Phys. A 767, 206.

Harada, T., Y. Hirabayashi, and A. Umeya, 2010, Phys. Lett. B 690, 363.

Harada, T., S. Shinmura, Y. Akaishi, and H. Tanaka, 1990, Nucl. Phys. A 507, 715.

Harada, T., A. Umeya, and Y. Hirabayashi, 2009, Phys. Rev. C 79, 014603.

Hasegawa, T., et al., 1996, Phys. Rev. C 53, 1210.

Hashimoto, O., and H. Tamura, 2006, Prog. Part. Nucl. Phys. $\mathbf{5 7}, 564$.

Hashimoto, O., et al., 1998, Nucl. Phys. A 639, 93c.

Hashimoto, T., et al., 2015, Prog. Theor. Exp. Phys. 2015, $061 \mathrm{D} 01$.

Hayano, R., T. Ishikawa, M. Iwasaki, H. Outa, E. Takada, H. Tamura, A. Sakaguchi, M. Aoki, and T. Yamazaki, 1989, Phys. Lett. B 231, 355.

Hell, T., and W. Weise, 2014, Phys. Rev. C 90, 045801.

von Hippel, F., 1964, Phys. Rev. 136, B455.

Hiyama, E., M. Kamimura, K. Miyazaki, and T. Motoba, 1999, Phys. Rev. C 59, 2351.

Hiyama, E., M. Kamimura, T. Motoba, T. Yamada, and Y. Yamamoto, 2000, Phys. Rev. Lett. 85, 270.

Hiyama, E., M. Kamimura, T. Motoba, T. Yamada, and Y. Yamamoto, 2001, Phys. Rev. C 65, 011301(R).

Hiyama, E., M. Kamimura, T. Motoba, T. Yamada, and Y. Yamamoto, 2002, Phys. Rev. C 66, 024007, and earlier work cited therein.

Hiyama, E., M. Kamimura, Y. Yamamoto, and T. Motoba, 2010a, Phys. Rev. Lett. 104, 212502.

Hiyama, E., T. Motoba, and Y. Yamamoto, 2010, Eds., Hypernuclei and baryon-baryon interaction, Prog. Theor. Phys. Suppl. 185, 1-343.

Hiyama, E., S. Ohnishi, B. F. Gibson, and T. A. Rijken, 2014, Phys. Rev. C 89, 061302(R).

Hiyama, E., S. Ohnishi, M. Kamimura, and Y. Yamamoto, 2013, Nucl. Phys. A 908, 29.

Hiyama, E., and T. Yamada, 2009, Prog. Part. Nucl. Phys. 63, 339 .

Hiyama, E., Y. Yamamoto, T. Motoba, and T. A. Rijken, 2008, Phys. Rev. C 78, 054316.

Holzenkamp, B., K. Holinde, and J. Speth, 1989, Nucl. Phys. A 500, 485.

Hosomi, K., et al., 2015, Prog. Theor. Exp. Phys. 2015, $081 \mathrm{D} 01$.

Hotchi, H., et al., 2001, Phys. Rev. C 64, 044302.

Hüfner, J., S. Y. Lee, and H. A. Weidenmüller, 1974, Nucl. Phys. A 234, 429.

Hungerford, E. V., 1994, Prog. Theor. Phys. Suppl. 117, 135.

Hungerford, E. V., and L. C. Biedenharn, 1984, Phys. Lett. B 142, 232.

Hyodo, T., 2013, Nucl. Phys. A 914, 260.

Hyodo, T., and W. Weise, 2008, Phys. Rev. C 77, 035204.

Ichikawa, A., et al., 2001, Phys. Lett. B 500, 37.

Ichikawa, Y., et al., 2014, Prog. Theor. Exp. Phys. 2014, $101 \mathrm{D} 03$. 
Ichikawa, Y., et al., 2015, Prog. Theor. Exp. Phys. 2015, $021 \mathrm{D} 01$.

Ikeda, K., T. Fukuda, T. Motoba, M. Takahashi, and Y. Yamamoto, 1994, Prog. Theor. Phys. 91, 747.

Ikeda, Y., T. Hyodo, and W. Weise, 2011, Phys. Lett. B 706, 63.

Ikeda, Y., T. Hyodo, and W. Weise, 2012, Nucl. Phys. A 881, 98.

Ikeda, Y., H. Kamano, and T. Sato, 2010, Prog. Theor. Phys. 124, 533 .

Ikeda, Y., and T. Sato, 2007, Phys. Rev. C 76, 035203.

Ikeda, Y., and T. Sato, 2009, Phys. Rev. C 79, 035201.

Inoue, T., et al., 2011, Phys. Rev. Lett. 106, 162002.

Inoue, T., et al., 2012, Nucl. Phys. A 881, 28.

Iodice, M., et al., 2007, Phys. Rev. Lett. 99, 052501.

Itonaga, K., and T. Motoba, 2010, Prog. Theor. Phys. Suppl. $185,252$.

Itonaga, K., T. Motoba, and H. Bandō, 1988, Z. Phys. A 330, 209.

Itonaga, K., T. Motoba, and H. Bandō, 1990, Prog. Theor. Phys. 84, 291.

Itonaga, K., T. Motoba, and M. Sotona, 1994, Prog. Theor. Phys. Suppl. 117, 17.

Itonaga, K., T. Motoba, T. Ueda, and T. A. Rijken, 2008, Phys. Rev. C 77, 044605.

Itonaga, K., T. Ueda, and T. Motoba, 2002, Phys. Rev. C 65, 034617.

Jido, D., E. Oset, and J. E. Palomar, 2001, Nucl. Phys. A 694, 525 .

Juliá-Diaz, B., V. Magas, E. Oset, A. Parreño, A. Polls, L. Tolós, I. Vidaña, and A. Ramos, 2013, Eds., Proceedings of the 11th international conference on hypernuclear and strange particle physics, Nucl. Phys. A 914, 1-568.

Jurič, M., et al., 1972, Nucl. Phys. B 47, 36.

Jurič, M., et al., 1973, Nucl. Phys. B 52, 1.

Kahana, D. E., S. H. Kahana, and D. J. Millener, 2003, Phys. Rev. C 68, 037302.

Kaiser, H., 2005, Phys. Rev. C 71, 068201.

Kaiser, N., P. B. Siegel, and W. Weise, 1995, Nucl. Phys. A $\mathbf{5 9 4}, 325$.

Kamada, H., J. Golak, K. Miyagawa, H. Witala, and W. Glöckle, 1998, Phys. Rev. C 57, 1595.

Kameoka, S., et al., 2005, Nucl. Phys. A 754, 173c.

Kang, B. H., et al., 2006, Phys. Rev. Lett. 96, 062301.

Kaplan, D. B., and A. E. Nelson, 1986, Phys. Lett. B 175, 57.

Karplus, R., and L. S. Rodberg, 1959, Phys. Rev. 115, 1058.

Karplus, R., and M. Ruderman, 1956, Phys. Rev. 102, 247.

Keil, C. M., F. Hofmann, and H. Lenske, 2000, Phys. Rev. C 61, 064309.

Kerman, A. K., and M. S. Weiss, 1973, Phys. Rev. C 8, 408.

Keyes, G., J. Sacton, J. H. Wickens, and M. M. Block, 1973, Nucl. Phys. B 67, 269.

Keyes, G., et al., 1970, Phys. Rev. D 1, 66.

Khaustov, P., et al., 2000a, Phys. Rev. C 61, 054603.

Khaustov, P., et al., 2000b, Phys. Rev. C 61, 027601.

Kielczewska, D., D. Ziemińska, and R. Dalitz, 1980, Nucl. Phys. A 333, 367.

Kielczewska, D., et al., 1975, Nucl. Phys. A 238, 437.

Kim, M., et al., 2009, Phys. Rev. Lett. 103, 182502.

Kim, M. J., et al., 2006, Phys. Lett. B 641, 28.

Kishimoto, T., 1986, Nucl. Phys. A 450, 447c.

Knorren, R., M. Prakash, and P. J. Ellis, 1995, Phys. Rev. C $\mathbf{5 2}, 3470$.
Kohno, M., 2010, Phys. Rev. C 81, 014003.

Kohno, M., and Y. Fujiwara, 2009, Phys. Rev. C 79, 054318.

Kohno, M., Y. Fujiwara, T. Fujita, C. Nakamoto, and Y. Suzuki, 2000, Nucl. Phys. A 674, 229.

Kohno, M., Y. Fujiwara, Y. Watanabe, K. Ogata, and M. Kawai, 2004, Prog. Theor. Phys. 112, 895.

Kohno, M., Y. Fujiwara, Y. Watanabe, K. Ogata, and M. Kawai, 2006, Phys. Rev. C 74, 064613.

Kohri, H., et al., 2002, Phys. Rev. C 65, 034607.

Krejčiřík, V., A. Cieplý, and A. Gal, 2010, Phys. Rev. C 82, 024609.

Kulessa, P., et al., 1998, Phys. Lett. B 427, 403.

Kumagai-Fuse, I., T. Koike, and Y. Akaishi, 1995, Nucl. Phys. A 585, 367c.

Kumagai-Fuse, I., and S. Okabe, 2002, Phys. Rev. C 66, 014003.

Lanskoy, D. E., and Y. Yamamoto, 2004, Phys. Rev. C 69, 014303.

Lattimer, J. M., 2012, Annu. Rev. Nucl. Part. Sci. 62, 485.

Leahy, D. A., et al., 2011, Astrophys. J. 742, 17.

Likar, A., M. Rosina, and B. Povh, 1986, Z. Phys. A 324, 35.

Lonardoni, D., A. Lovato, S. Gandolfi, and F. Pederiva, 2015, Phys. Rev. Lett. 114, 092301.

Lonardoni, D., F. Pederiva, and S. Gandolfi, 2014, Phys. Rev. C 89, 014314.

Ma, Y., et al., 2010, Nucl. Phys. A 835, 422.

Maessen, P. M. M., T. A. Rijken, and J. J. de Swart, 1989, Phys. Rev. C 40, 2226.

Magas, V. K., E. Oset, A. Ramos, and H. Toki, 2006, Phys. Rev. C 74, 025206.

Mareš, J., E. Friedman, and A. Gal, 2006, Nucl. Phys. A 770, 84.

Mareš, J., E. Friedman, A. Gal, and B. K. Jennings, 1995, Nucl. Phys. A 594, 311.

Mareš, J., and B. K. Jennings, 1994, Phys. Rev. C 49, 2472.

Maruta, T., et al., 2007, Eur. Phys. J. A 33, 255.

Matsuyama, A., and K. Yazaki, 1988, Nucl. Phys. A 477, 673.

May, M., et al., 1981, Phys. Rev. Lett. 47, 1106.

May, M., et al., 1983, Phys. Rev. Lett. 51, 2085.

McCracken, M. E., M. Bellis, C. A. Meyer, and M. Williams, 2010, Phys. Rev. C 81, 025201.

Merrill, F., et al., 2001, Phys. Rev. C 63, 035206.

Millener, D. J., 1990, in LAMPF workshop on (pi,K) physics, edited by B. F. Gibson, W. R. Gibbs, and M. B. Johnson, AIP Conf. Proc. Vol. 224 (AIP, New York), p. 128.

Millener, D. J., 2005, Nucl. Phys. A 754, 48c.

Millener, D. J., 2007, in Topics in Strangeness $\mathrm{Nu}$ clear Physics, edited by P. Bydžovský, A. Gal, and J. Mareš, Lecture Notes in Physics, Vol. 724 (Springer, Berlin/Heidelberg), p. 31.

Millener, D. J., 2008, Nucl. Phys. A 804, 84.

Millener, D. J., 2010, Nucl. Phys. A 835, 11.

Millener, D. J., 2012, Nucl. Phys. A 881, 298.

Millener, D. J., C. B. Dover, and A. Gal, 1988, Phys. Rev. C 38, 2700 .

Millener, D. J., C. B. Dover, and A. Gal, 1994, Prog. Theor. Phys. Suppl. 117, 307.

Millener, D. J., A. Gal, C. B. Dover, and R. H. Dalitz, 1985, Phys. Rev. C 31, 499.

Milner, C., et al., 1985, Phys. Rev. Lett. 54, 1237.

Miura, Y., 2005, Gamma-ray spectroscopy of L11B and L10B, Ph.D. thesis, Tohoku University, Sendai, Japan.

Miyagawa, K., H. Kamada, W. Glöckle, and V. Stoks, 1995, Phys. Rev. C 51, 2905. 
Montwill, A., et al., 1974, Nucl. Phys. A 234, 413.

Morita, K., T. Furumoto, and A. Ohnishi, 2015, Phys. Rev. C 91, 024916.

Morris, C. L., et al., 1989, NMS Collaboration, Proposal for a High Resolution Spectrometer for Neutral Mesons, Los Alamos National Laboratory, (unpublished).

Motoba, T., Y. Akaishi, and K. Ikeda, 1994, Eds., Developments in hypernuclear physics, Prog. Theor. Phys. Suppl. 117, $1-528$.

Motoba, T., H. Bandō, R. Wünsch, and J. Žofka, 1988, Phys. Rev. C 38, 1322.

Motoba, T., and K. Itonaga, 1994, Prog. Theor. Phys. Suppl. $\mathbf{1 1 7}, 477$.

Motoba, T., K. Itonaga, and Y. Yamamoto, 2010, Prog. Theor. Phys. Suppl. 185, 197.

Motoba, T., D. E. Lanskoy, D. J. Millener, and Y. Yamamoto, 2008, Nucl. Phys. A 804, 99.

Motoba, T., M. Sotona, and K. Itonaga, 1994b, Prog. Theor. Phys. Suppl. 117, 123.

Nagae, T., 2001, Nucl. Phys. A 691, 76c.

Nagae, T., 2007, in Topics in Strangeness Nuclear Physics, edited by P. Bydžovský, A. Gal, and J. Mareš, Lecture Notes in Physics, Vol. 724 (Springer, Berlin/Heidelberg), p. 81.

Nagae, T., 2013, Few-Body Syst. 54, 785.

Nagae, T., et al., 1998, Phys. Rev. Lett. 80, 1605.

Nagels, M. M., T. A. Rijken, and J. J. de Swart, 1977, Phys. Rev. D 15, 2547.

Nagels, M. M., T. A. Rijken, and J. J. de Swart, 1979, Phys. Rev. D 20, 1633.

Nagels, M. M., T. A. Rijken, and Y. Yamamoto, 2015a, arXiv:1504.02634.

Nagels, M. M., T. A. Rijken, and Y. Yamamoto, 2015b, arXiv:1501.06636.

Nakamura, S. N., 2013, Nucl. Phys. A 914, 3.

Nakano, T., and K. Nishijima, 1953, Prog. Theor. Phys. 10, 581.

Nakazawa, K., et al., 2015, Prog. Theor. Exp. Phys. 2015, $033 \mathrm{D} 02$.

Nemura, H., Y. Akaishi, and K. S. Myint, 2003, Phys. Rev. C 67, 051001(R).

Nemura, H., Y. Akaishi, and Y. Suzuki, 2002, Phys. Rev. Lett. 89, 142504.

Nemura, H., S. Shinmura, Y. Akaishi, and K. S. Myint, 2005, Phys. Rev. Lett. 94, 202502.

Nield, K. J., et al., 1976, Phys. Rev. C 13, 1263.

Nieves, J., and E. Oset, 1993, Phys. Rev. C 47, 1478.

Niiyama, M., 2013, Nucl. Phys. A 914, 543.

Noga, V. I., et al., 1986, Sov. J. Nucl. Phys. 43, 856.

Nogga, A., 2013, Nucl. Phys. A 914, 140.

Nogga, A., H. Kamada, and W. Glöckle, 2002, Phys. Rev. Lett. 88, 172501.

Noumi, H., et al., 2002, Phys. Rev. Lett. 89, 072301.

Noumi, H., et al., 2003, Phys. Rev. Lett. 90, 049902(E).

Okada, S., et al., 2004, Phys. Lett. B 597, 249.

Okada, S., et al., 2005, Nucl. Phys. A 754, 178c.

Olive, K. A., et al., 2014, Chin. Phys. C 38, 090001.

Oppenheimer, J. R., and G. M. Volkoff, 1939, Phys. Rev. 55, 374.

Oset, E., P. Fernández de Córdoba, J. Nieves, A. Ramos, and

L. L. Salcedo, 1994, Prog. Theor. Phys. Suppl. 117, 461.

Oset, E., and A. Ramos, 1998, Nucl. Phys. A 635, 99.

Oset, E., and L. L. Salcedo, 1985, Nucl. Phys. A 443, 704.

Park, H., et al., 2000, Phys. Rev. C 61, 054004.
Parker, J. D., et al., 2007, Phys. Rev. C 76, 035501.

Parreño, A., 2007, in Topics in Strangeness Nuclear Physics, edited by P. Bydžovský, A. Gal, and J. Mareš, Lecture Notes in Physics, Vol. 724 (Springer, Berlin/Heidelberg), p. 141.

Parreño, A., C. Bennhold, and B. R. Holstein, 2004, Phys. Rev. C 70, 051601(R).

Parreño, A., C. Bennhold, and B. R. Holstein, 2005, Nucl. Phys. A 754, 127c.

Parreño, A., and A. Ramos, 2001, Phys. Rev. C 65, 015204.

Parreño, A., A. Ramos, and C. Bennhold, 1997, Phys. Rev. C 56, 339.

Petschauer, S., N. Kaiser, J. Haidenbauer, U.-G. Meißner, and W. Weise, 2016, Phys. Rev. C 93, 014001.

Piekarz, H., et al., 1982, Phys. Lett. B 110, 428.

Pile, P., et al., 1991, Phys. Rev. Lett. 66, 2585.

Pochodzalla, J., 2005, Nucl. Phys. A 754, 430c.

Pochodzalla, J., and T. Walcher, 2007, Eds., Proceedings of the 9th international conference on hypernuclear and strange particle physics (SIF and Springer-Verlag Berlin/Heidelberg), pp. 1-406.

Polinder, H., J. Haidenbauer, and U.-G. Meißner, 2006, Nucl. Phys. A 779, 244.

Pop, T., and S. D. Gupta, 2010, Phys. Rev. C 81, 054911.

Poulard, G., A. Givernaud, and A. Borg, 1973, Phys. Lett. B 46, 135.

Povh, B., 1980, Nucl. Phys. A 335, 233.

Prem, R. J., and P. H. Steinberg, 1964, Phys. Rev. 136, B1803.

Prowse, D. J., 1966, Phys. Rev. Lett. 17, 782.

Pysz, K., et al., 1999, Nucl. Inst. Meth. Phys. Res. A 420, 356.

Randeniya, S. D., and E. V. Hungerford, 2007, Phys. Rev. C 76, 064308.

Rappold, C., et al., 2013a, Nucl. Phys. A 913, 170.

Rappold, C., et al., 2013b, Phys. Rev. C 88, 041001(R).

Rappold, C., et al., 2014, Phys. Lett. B 728, 543.

Rappold, C., et al., 2015, Phys. Lett. B 747, 129.

Rayet, M., and R. H. Dalitz, 1966, Nuovo Cim. A 46, 786.

Reuber, A., K. Holinde, and J. Speth, 1994, Nucl. Phys. A $\mathbf{5 7 0}, 543$.

Révai, J., and N. Shevchenko, 2014, Phys. Rev. C 90, 034004.

Rijken, T. A., M. M. Nagels, and Y. Yamamoto, 2010, Nucl. Phys. A 835, 160.

Rijken, T. A., M. M. Nagels, and Y. Yamamoto, 2013, FewBody Syst 54, 801.

Rijken, T. A., and H.-J. Schulze, 2016, Eur. Phys. J. A 52, 21.

Rijken, T. A., V. J. G. Stoks, and Y. Yamamoto, 1999, Phys. Rev. C 59, 21.

Rijken, T. A., and Y. Yamamoto, 2006a, Phys. Rev. C 73, 044008.

Rijken, T. A., and Y. Yamamoto, 2006b, arXiv:nucl.

Sada, Y., et al., 2016, Prog. Theor. Exp. Phys. 2016, $051 D 01$.

Saha, P. K., et al., 2004, Phys. Rev. C 70, 044613.

Saha, P. K., et al., 2005, Phys. Rev. Lett. 94, 052502.

Saito, K., M. Oka, and T. Suzuki, 1997, Nucl. Phys. A 625 , 95.

Sakata, S., 1956, Prog. Theor. Phys. 16, 686.

Sano, M., M. Wakai, and Y. Yamamoto, 1992, Prog. Theor. Phys. 87, 957.

Sasaki, K., M. Izaki, and M. Oka, 2005, Phys. Rev. C 71, 035502 .

Sasao, J., et al., 2004, Phys. Lett. B 579, 258. 
Sato, Y., et al., 2005, Phys. Rev. C 71, 025203.

Schaffner, J., C. B. Dover, A. Gal, C. Greiner, and H. Stöcker, 1993, Phys. Rev. Lett. 71, 1328.

Schaffner, J., C. B. Dover, A. Gal, D. J. Millener, C. Greiner, and H. Stöcker, 1994, Ann. Phys. (N.Y.) 235, 35.

Schaffner, J., M. Hanauske, H. Stöcker, and W. Greiner, 2002, Phys. Rev. Lett. 89, 171101.

Schaffner-Bielich, J., 2008, Nucl. Phys. A 804, 309.

Schaffner-Bielich, J., and A. Gal, 2000, Phys. Rev. C 62, 034311, and references cited therein.

Schulze, H.-J., A. Polls, A. Ramos, and I. Vidaña, 2006, Phys. Rev. C 73, 058801.

Schulze, H.-J., and T. A. Rijken, 2011, Phys. Rev. C 84, 035801.

Sechi-Zorn, B., B. Kehoe, J. Twitty, and R. A. Burnstein, 1968, Phys. Rev. 175, 1735.

Shevchenko, N., A. Gal, and J. Mareš, 2007a, Phys. Rev. Lett. 98, 082301.

Shevchenko, N., A. Gal, J. Mareš, and J. Révai, 2007b, Phys. Rev. C 76, 044004.

Shevchenko, N. V., and J. Haidenbauer, 2015, Phys. Rev. C 92, 044001.

Shyam, R., K. Tsushima, and A. W. Thomas, 2012, Nucl. Phys. A 881, 255.

Skoupil, D., and P. Bydžovský, 2016, Phys. Rev. C 93, 025204.

Sotona, M., and S. Frullani, 1994, Prog. Theor. Phys. Suppl. $\mathbf{1 1 7}, 151$.

Steinheimer, J., K. Gudima, A. Botvina, I. Mishustin, M. Bleicher, and H. Stöcker, 2012, Phys. Lett. B 714, 85.

Stoks, V. G. J., and T. A. Rijken, 1999, Phys. Rev. C 59, 3009.

Stotzer, R. W., et al., 1997, Phys. Rev. Lett. 78, 3646.

Sugimura, H., et al., 2014, Phys. Lett. B 729, 39.

Suzuki, T., et al., 2004, Phys. Lett. B 597, 263.

Suzuki, T., et al., 2005, Nucl. Phys. A 754, 375c.

Takahashi, H., et al., 2001, Phys. Rev. Lett. 87, 212502.

Takahashi, T., 2013, Nucl. Phys. A 914, 530.

Tamura, H., 2010, Prog. Theor. Phys. Suppl. 185, 315.

Tamura, H., R. S. Hayano, H. Outa, and T. Yamazaki, 1994, Prog. Theor. Phys. Suppl. 117, 1.

Tamura, H., et al., 1989, Phys. Rev. C 40, R479.

Tamura, H., et al., 2000, Phys. Rev. Lett. 84, 5963.

Tamura, H., et al., 2005, Nucl. Phys. A 754, 58c.

Tamura, H., et al., 2013, Nucl. Phys. A 914, 99.

Tang, L., et al., 1988, Phys. Rev. C 38, 846.

Tang, L., et al., 2014, Phys. Rev. C 90, 034320.

Tang, Y. C., and R. C. Herndon, 1965, Phys. Rev. Lett. 14, 991.

Tanida, K., et al., 2001, Phys. Rev. Lett. 86, 1982.

Thiessen, H. A., et al., 1980, AGS Proposal \# 738, Brookhaven National Laboratory.

Thorsett, S. E., and D. Chakrabarty, 1999, Astrophys. J. 512, 288.

Tokiyasu, A. O., et al., 2014, Phys. Lett. B 728, 616.

Tolman, R. C., 1939, Phys. Rev. 55, 364.

Tomozawa, Y., 1966, Nuovo Cimento 46, 707.

Uchino, T., T. Hyodo, and M. Oka, 2011, Nucl. Phys. A 868$\mathbf{8 6 9}, 53$.

Ukai, M., 2004, Study of spin-dependent LN interactions by gamma-ary spectroscopy of $p$-shell hypernuclei, $\mathrm{Ph} . \mathrm{D}$. thesis, Tohoku University, Sendai, Japan.

Ukai, M., et al., 2004, Phys. Rev. Lett. 93, 232501.

Ukai, M., et al., 2006, Phys. Rev. C 73, 012501(R).
Ukai, M., et al., 2008, Phys. Rev. C 77, 054315.

Umeya, A., and T. Harada, 2009, Phys. Rev. C 79, 024315.

Umeya, A., and T. Harada, 2011, Phys. Rev. C 83, 034310.

Urciuoli, G. M., et al., 2015, Phys. Rev. C. 91, 034308.

Usmani, Q. N., and A. R. Bodmer, 1999, Phys. Rev. C 60, 055215 .

Velde-Wilquet, C. V., J. Sacton, J. H. Wickens, D. N. Tovee, and D. H. Davis, 1977, Nuovo Cimento A 39, 538.

Vidaña, I., D. Logoteta, C. Providência, A. Polls, and I. Bombaci, 2011, Europhys. Lett. 94, 11002.

Vidaña, I., A. Polls, A. Ramos, and H.-J. Schulze, 2001, Phys. Rev. C 64, 044301.

Wang, X. C., H. Takaki, and H. Bandō, 1986, Prog. Theor. Phys. 76, 865.

Watson, K. M., 1952, Phys. Rev. 88, 1163.

Weinberg, S., 1966, Phys. Rev. Lett. 17, 616.

Weise, W., 2015, Hyp. Int. 233, 131.

Weise, W., and R. Härtle, 2008, Nucl. Phys. A 804, 173.

Wiegand, C. E., and D. A. Mack, 1967, Phys. Rev. Lett. 18, 685.

Wirth, R., D. Gazda, P. Navrátil, A. Calci, J. Langhammer, and R. Roth, 2014, Phys. Rev. Lett. 113, 192502.

Witten, E., 1984, Phys. Rev. D 30, 272.

Wycech, S., and A. Green, 2009, Phys. Rev. C 79, 014001.

Xu, G., and E. V. Hungerford, 2003, Nucl. Instrum. Methods A 501, 602.

Yamada, T., and K. Ikeda, 1997, Phys. Rev. C 56, 3216.

Yamamoto, T. O., et al., 2015, Phys. Rev. Lett. 115, 222501.

Yamamoto, Y., 1995a, in Nuclear and Particle Physics with Meson Beams in the $1 \mathrm{GeV} / \mathrm{c}$ Region, edited by S. Sugimoto and O. Hashimoto (Universal Academy Press, Inc., Tokyo), p. 267.

Yamamoto, Y., 1995b, Few-Body Syst. Suppl. 9, 145.

Yamamoto, Y., H. Bandō, and J. Žofka, 1988, Prog. Theor. Phys. 80, 757.

Yamamoto, Y., T. Furumoto, N. Yasutake, and T. A. Rijken, 2013, Phys. Rev. C 88, 022801.

Yamamoto, Y., T. Furumoto, N. Yasutake, and T. A. Rijken, 2014, Phys. Rev. C 90, 045805.

Yamamoto, Y., T. Furumoto, N. Yasutake, and T. A. Rijken, 2016, Eur. Phys. J. A 52, 19.

Yamamoto, Y., T. Motoba, H. Himeno, K. Ikeda, and S. Nagata, 1994a, Prog. Theor. Phys. Suppl. 117, 361.

Yamamoto, Y., T. Motoba, and T. A. Rijken, 2010, Prog. Theor. Phys. Suppl. 185, 72.

Yamamoto, Y., and T. A. Rijken, 2008, Nucl. Phys. A 804, 139.

Yamamoto, Y., and T. A. Rijken, 2013, Few-Body Syst. 54, 57.

Yamamoto, Y., M. Sano, and M. Wakai, 1994b, Prog. Theor. Phys. Suppl. 117, 265.

Yamamoto, Y., H. Takaki, and K. Ikeda, 1991, Prog. Theor. Phys. 86, 867.

Yamamoto, Y., M. Wakai, T. Fukuda, and M. Sano, 1992, Prog. Theor. Phys. 88, 1163.

Yamamoto, Y., M. Wakai, T. Motoba, and T. Fukuda, 1997, Nucl. Phys. A 625, 107.

Yamazaki, T., and Y. Akaishi, 2002, Phys. Lett. B 535, 70.

Yamazaki, T., et al., 1985, Phys. Rev. Lett. 54, 102.

Yamazaki, T., et al., 2010, Phys. Rev. Lett. 104, 132502.

Zech, G., et al., 1977, Nucl. Phys. B. 124, 413.

Zhu, D., C. B. Dover, A. Gal, and M. May, 1991, Phys. Rev. Lett. 67, 2268.

Ziemińska, D., 1975, Nucl. Phys. A 242, 461. 
Ziemińska, D., and R. H. Dalitz, 1975, Nucl. Phys. A 238, 453. 